\title{
JV TASK 99 - INTEGRATED RISK ANALYSIS AND CONTAMINANT REDUCTION, WATFORD CITY, NORTH DAKOTA
}

Final Report

Submitted to:

AAD Document Control

U.S. Department of Energy

National Energy Technology Laboratory

PO Box 10940, MS 921-107

Pittsburgh, PA 15236-0940

Cooperative Agreement No.: DE-FC26-98FT40321

Project Manager: Eugene Ashby

Prepared by:

Jaroslav Solc Barry W. Botnen

Energy \& Environmental Research Center University of North Dakota 15 North 23rd Street, Stop 9018

Grand Forks, North Dakota 58202-9018 


\section{DISCLAIMER}

This report was prepared as an account of work sponsored by an agency of the United States Government. Neither the United States Government, nor any agency thereof, nor any of their employees makes any warranty, express or implied, or assumes any legal liability or responsibility for the accuracy, completeness, or usefulness of any information, apparatus, product, or process disclosed or represents that its use would not infringe privately owned rights. Reference herein to any specific commercial product, process, or service by trade name, trademark, manufacturer, or otherwise does not necessarily constitute or imply its endorsement, recommendation, or favoring by the United States Government or any agency thereof. The views and opinions of authors expressed herein do not necessarily state or reflect those of the United States Government or any agency thereof.

This report is available to the public from the National Technical Information Service, U.S. Department of Commerce, 5285 Port Royal Road, Springfield, VA 22161; phone orders accepted at (703) 487-4650.

\section{EERC DISCLAIMER}

LEGAL NOTICE This research report was prepared by the Energy \& Environmental Research Center (EERC), an agency of the University of North Dakota, as an account of work sponsored by the U.S. Department of Energy (DOE). Because of the research nature of the work performed, neither the EERC nor any of its employees makes any warranty, express or implied, or assumes any legal liability or responsibility for the accuracy, completeness, or usefulness of any information, apparatus, product, or process disclosed or represents that its use would not infringe privately owned rights. Reference herein to any specific commercial product, process, or service by trade name, trademark, manufacturer, or otherwise does not necessarily constitute or imply its endorsement or recommendation by the EERC. 


\title{
JV TASK 99 - INTEGRATED RISK ANALYSIS AND CONTAMINANT REDUCTION, WATFORD CITY, NORTH DAKOTA
}

\begin{abstract}
The Energy \& Environmental Research Center (EERC) conducted a limited site investigation and risk analyses for hydrocarbon-contaminated soils and groundwater at a Construction Services, Inc., site in Watford City, North Dakota. Site investigation confirmed the presence of free product and high concentrations of residual gasoline-based contaminants in several wells, the presence of 1,2-dichloroethane, and extremely high levels of electrical conductivity indicative of brine residuals in the tank area south of the facility.

The risk analysis was based on compilation of information from the site-specific geotechnical investigation, including multiphase extraction pilot test, laser induced fluorescence probing, evaluation of contaminant properties, receptor survey, capture zone analysis and evaluation of well head protection area for municipal well field. The project results indicate that the risks associated with contaminant occurrence at the Construction Services, Inc. site are low and, under current conditions, there is no direct or indirect exposure pathway between the contaminated groundwater and soils and potential receptors.
\end{abstract}




\section{TABLE OF CONTENTS}

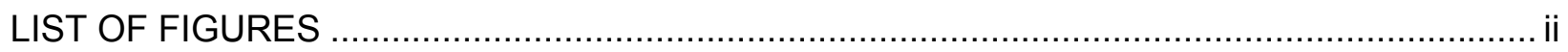

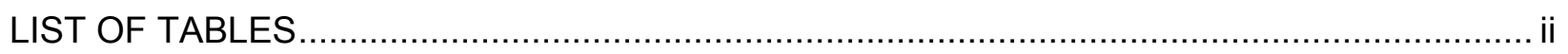

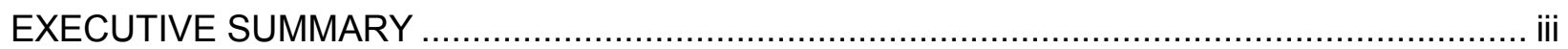

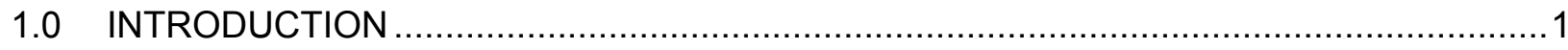

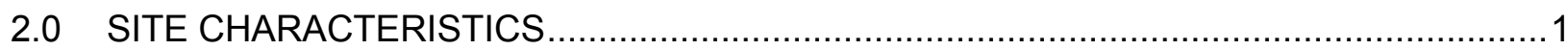

2.1 Site Location and Contaminant Release History............................................ 1

2.2 Supplementary Site Investigation and Sediment Properties ................................. 1

2.3 Geology and Hydrogeology...........................................................................

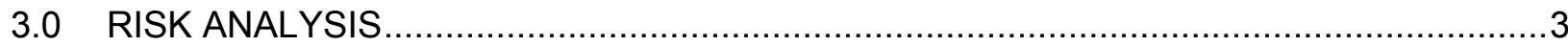

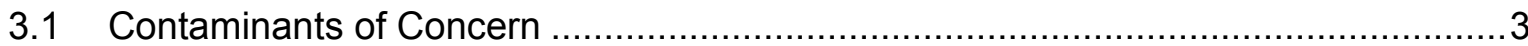

3.1.1 Selected Properties ......................................................................

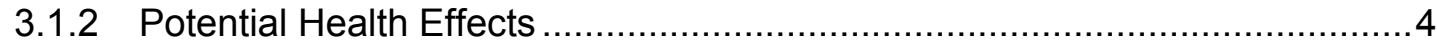

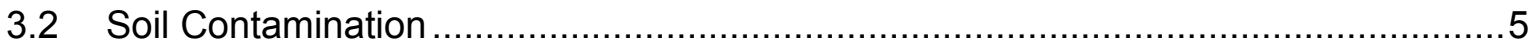

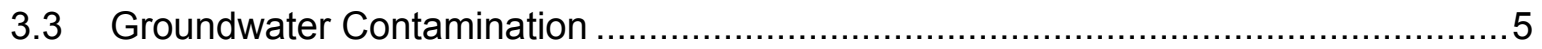

3.4 Hydraulic Properties and Contaminant Transport ..............................................

3.5 Municipal Well - Capture Zone Analysis ..........................................................

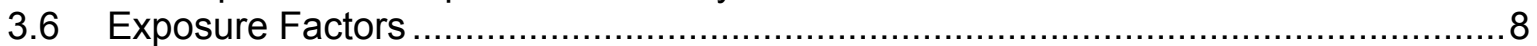

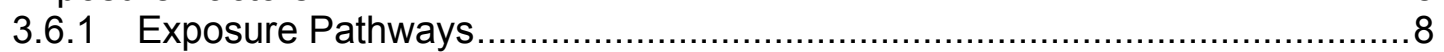

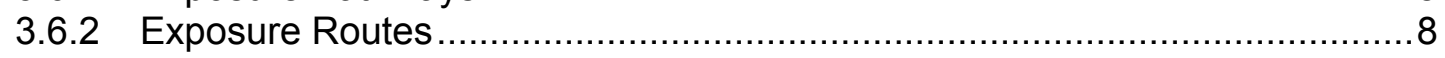

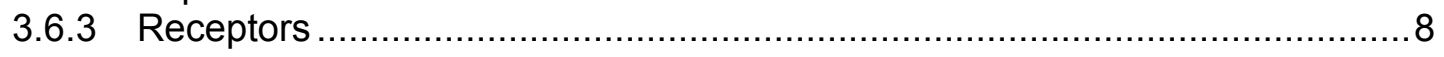

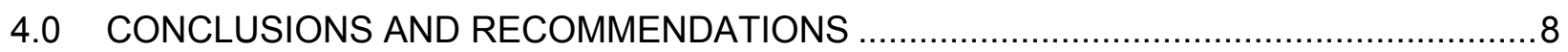

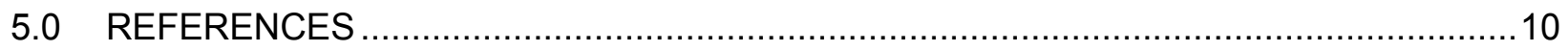

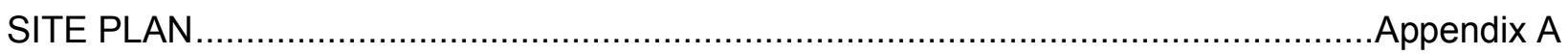

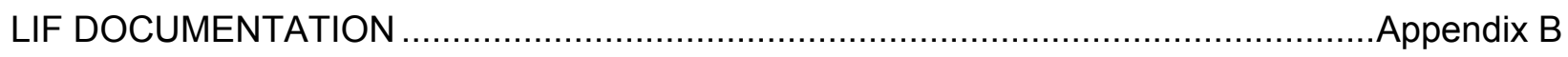

GROUNDWATER TABLE MONITORING …………….................................. Appendix C

RESULTS OF HYDRAULIC TESTING .......................................................... Appendix D

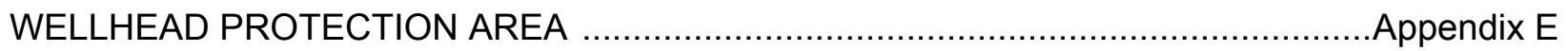

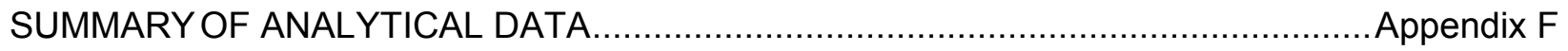

COMPLETE ANALYTICAL DOCUMENTATION ………..................................... Appendix G GROUNDWATER ANALYSES - COC ................................................... Appendix G-1

BIODEGRADATION INDICATORS ...................................................... Appendix G-2 


\section{LIST OF FIGURES}

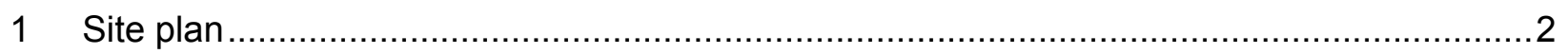

\section{LIST OF TABLES}

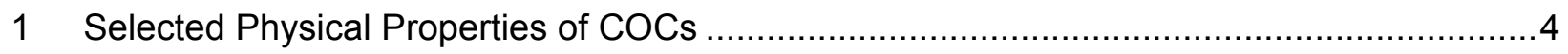

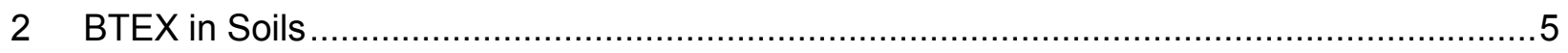

3 COC Concentrations in Groundwater (analyses - December 6, 2006) .......................6 


\section{JV TASK 99 - INTEGRATED RISK ANALYSIS AND CONTAMINANT REDUCTION, WATFORD CITY, NORTH DAKOTA}

\section{EXECUTIVE SUMMARY}

The Energy \& Environmental Research Center (EERC) conducted a limited site investigation and risk analysis for hydrocarbon-contaminated soils and groundwater at a Construction Services, Inc., site in Watford City, North Dakota. Site investigation confirmed the presence of free product and high concentrations of residual gasoline-based contaminants in several wells, the presence of 1,2-dichloroethane, and extremely high levels of electrical conductivity indicative of brine residuals in the tank area south of the facility.

The risk analysis was based on compilation of information from the site-specific geotechnical investigation, including a multiphase extraction pilot test, laser-induced fluorescence probing, evaluation of contaminant properties, a receptor survey, capture zone analysis, and evaluation of well head protection area for the municipal well field. The project results indicate that the risks associated with contaminant occurrence at the Construction Services, Inc., site are low and, under current conditions, there is no direct or indirect exposure pathway between the contaminated groundwater and soils and potential receptors. 


\section{JV TASK 99 - INTEGRATED RISK ANALYSIS AND CONTAMINANT REDUCTION, WATFORD CITY, NORTH DAKOTA}

\subsection{INTRODUCTION}

At the request of the North Dakota Department of Health (NDDH), the Energy \& Environmental Research Center (EERC) conducted a limited site investigation and risk analysis. The project primary objective was to integrate recently completed multiphase extraction (MPE) pilot testing (EERC) and a capture zone analysis for the nearby municipal well field (NDDH) into risk analysis for the contaminated area at the Construction Services, Inc., site in Watford City, North Dakota.

The summary of activities presented in this report is as follows:

- Supplemental site investigation including laser-induced fluorescence (LIF) GeoProbe by Dakota Technologies, Inc., conducted November 7-8, 2006.

- Groundwater sampling, water-table monitoring, and hydraulic testing conducted December 5-6, 2006.

- Evaluation of information from the municipal well field, including capture zone analysis and well head protection area.

- Evaluation of remedial technologies/strategies for the subject site.

\subsection{SITE CHARACTERISTICS}

\subsection{Site Location and Contaminant Release History}

The original source area is located at 1100 4th Avenue Northeast, T150N R98W Section 18, McKenzie County, Watford City, North Dakota. The current facility, operated by Construction Services, Inc., is a former Chinook Pipeline property. The inferred extent of impacted area is about $100 \times 300 \mathrm{ft}$. The impacted area is in proximity to the municipal well and Cherry Creek. The site layout is provided in Figure 1 and Appendix A.

Following the removal of nine underground storage tanks (USTs) south, west, and north of the former Chinook Pipeline property, the site has undergone monitoring since 1991 [1, 2]. A remedial investigation/feasibility study (RI/FS) was conducted by the EERC in 2005 and included MPE pilot testing and a limited site investigation [3].

\subsection{Supplementary Site Investigation and Sediment Properties}

An initial site investigation, focusing on the former south tank location was conducted as part of the FS in November 2005 [3]. Supplementary site characterization using GeoProbe and LIF technology was conducted November 7-8, 2006, focusing on the tank areas west and north of the facility. A total of 22 boreholes were advanced to depths of 18-22 ft in order to provide information on contaminant distribution within the sediment profile. Spikes of fluorescence intensity in a wide range of depths between 4 and $16 \mathrm{ft}$ below ground indicate the presence of 
LIF-detectable amounts of residual free product in thin layers conducive to product transport and retention. Expanded probing activity to tank areas west and north of the facility did not confirm the presence of free product. Based on probing results and intensity of the LIF response, residual free product is retained in thin discrete layers or lenses of sandy silt and silty sand within the smear zone of fluctuating groundwater. LIF profiles and borehole locations, including data from 2005 LIF activities, are provided in Appendix B.

It is important to note that LIF technology is limited to detection of petroleum hydrocarbons (not chlorinated compounds) in the free phase (not dissolved phase). Further limitations are associated with the tight sedimentary profile such as in clays and clayey silts. Because the extended LIF investigation did not identify contamination in the tank areas west and north of the facility, the proposed expansion of the groundwater-monitoring network was abandoned.

\section{EERC JS29496.CDR}

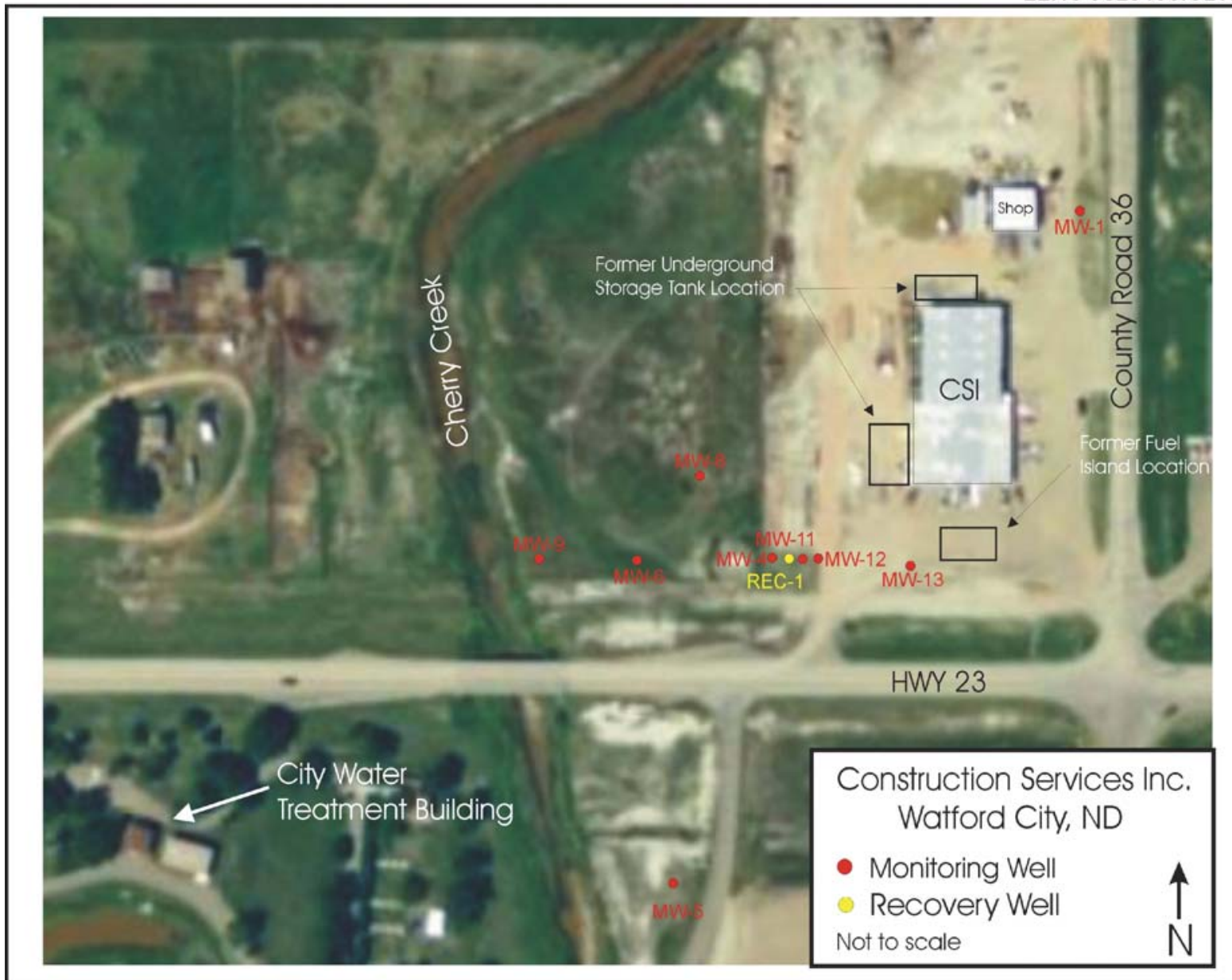

Figure 1. Site plan. 


\subsection{Geology and Hydrogeology}

The sediment profile of the impacted area is dominated by a heterogeneous complex of silty, sandy clays to 8-12 ft that overlay poorly graded silty sands to about $20 \mathrm{ft}$ (terminal depth of wells at the site). Based on geological documentation from a wider area (North Dakota State Water Commission [NDSWC] data and municipal well logs), the clays and lakebed deposits consist of silty clays with highly variable fractions of fine-grained silty sands that extend up to 30-50 ft belowground. These deposits are underlain by the poorly sorted sands and gravels of the Tobacco Garden Creek Aquifer. The top of the aquifer is documented at $34 \mathrm{ft}$ and $51 \mathrm{ft}$ below the surface for municipal wells No. $6(2750 \mathrm{ft} \mathrm{SW})$ and No. 8 (700 ft SW of the site), respectively.

Depth to water at the site varied from about 10 to $13 \mathrm{ft}$ below ground surface between December 12, 2005, and December 6, 2006 (Appendix C). Groundwater in the shallow intercepted profile is unconfined, with flow direction to the northwest toward Cherry Creek. Groundwater table fluctuation in a relatively shallow aquifer and the attendant thickness of the smear zone reflect the proximity of Cherry Creek, the sewage lift station, and leaking wellheads at older wells.

Groundwater in the Tobacco Garden Creek Aquifer is confined, with a static water table at $7 \mathrm{ft}$ belowground and prevailing groundwater flow direction to the north-northeast. A dynamic water table during intermittent pumping at city well No. 8 is maintained at about $40 \mathrm{ft}$ belowground. The natural water table in the confined Tobacco Garden Creek Aquifer fluctuated over $8 \mathrm{ft}$ between 1991 and 2004 based on NDSWC data from well No. 150-098-18 DDCD west of the site $[3,4]$.

Groundwater chemistry at the site is dominated by sodium, sulfate, and bicarbonate ions, with high concentrations of iron and electrical conductivity (EC) over $22 \mathrm{mS} / \mathrm{cm}$ (wells $\mathrm{MW}-1$, $\mathrm{MW}-8)$. Biodegradation parameters indicate limited in situ biodegradation reactions in a reducing environment (Appendix $F$ ).

Extremely high concentrations of selected parameters in relatively shallow monitoring wells are not typical for the Tobacco Garden Creek Aquifer pumped for the municipality. High EC and hardness could be associated with a natural discharge pattern. In such a scenario, intensive salt precipitation in upper parts of the sediment profile could be a result of upward flow of sodium and sulfate-rich groundwater, reflecting the confined nature of the underlying aquifer. With respect to unusually high values, however, EC and hardness may be indicative of contamination with brine residuals. Data from NDSWC well No. 150-098-18 DDCD (west of the site and north of the municipal well field) indicate almost a $50 \%$ increase in EC and total dissolved solids (TDS) between 1981 and 2000 [3].

\subsection{RISK ANALYSIS}

\subsection{Contaminants of Concern}

\subsubsection{Selected Properties}

The primary contaminants of concern are gasoline-based BTEX (benzene, toluene, ethylbenzene, and xylenes) and 1,2,4-trimethylbenzene. In addition, 1,2-dichloroethane was detected at the site. Its typically industrial use is as a solvent for oils, waxes, and resins; a lead 
scavenger in gasoline; and a fumigant. Benzene and 1,2-dichloroethane are recognized as carcinogens. Selected primary physical properties of contaminants of concern (COCs) are summarized in Table 1.

Table 1. Selected Physical Properties of COCs

\begin{tabular}{lccccc}
\hline & $\begin{array}{c}\text { Molecular } \\
\text { Weight }\end{array}$ & $\begin{array}{c}\text { Specific } \\
\text { Gravity }\end{array}$ & $\begin{array}{c}\text { Solubility } \\
\mathrm{mg} / \mathrm{l} \text { at } 20^{\circ} \mathrm{C}\end{array}$ & $\begin{array}{c}\text { Vapor Pressure at } \\
1 \mathrm{~atm}\left({ }^{\circ} \mathrm{C}\right)\end{array}$ & $\begin{array}{c}\text { Absorbability } \\
\mathrm{mg} / \mathrm{g}(\mathrm{at} \mathrm{ppb})\end{array}$ \\
\hline Benzene & 78 & 0.88 & 1780 & $76(20)$ & $80(416)$ \\
Toluene & 92 & 0.87 & 515 & $22(20)$ & $50(317)$ \\
Xylenes (3) & 106 & 0.88 & $540\left(25^{\circ} \mathrm{C}\right)$ & $6(20)$ & $70(500)$ \\
Ethylbenzene & 106 & 0.87 & 105 & $7(20)$ & $18(115)$ \\
1,2 Dichloroethane & 99 & 1.25 & $8300\left(25^{\circ} \mathrm{C}\right)$ & $87(25)$ & $2(500)$ \\
\hline
\end{tabular}

Data compiled from the U.S. Environmental Protection Agency (EPA) [5]; Merck Index, 1996 [6];

Suthersan, 2002 [7]; Nyer, 2001 [8]; and Montgomery and Welcom, 1990 [9].

\subsubsection{Potential Health Effects}

\subsubsection{Benzene}

Short-term: Benzene has the potential to cause the following health effects when people are exposed to it at levels above the maximum contaminant level (MCL) for relatively short periods of time: temporary nervous system disorders, immune system depression, and anemia.

Long-term: Benzene has the potential to cause the following effects from a lifetime exposure at levels above the MCL: chromosome aberrations and cancer.

\subsubsection{1,2-Dichloroethane}

Short-term: 1,2-dichloroethane has the potential to cause the following health effects when people are exposed to it at levels above the MCL for relatively short periods of time: central nervous system disorders, and adverse lung, kidney, liver, circulatory, and gastrointestinal effects.

Long-term: 1,2-dichloroethane has the potential to cause the following effects from a lifetime exposure at levels above the MCL: cancer.

\subsubsection{Ethylbenzene}

Short-term: Ethylbenzene has the potential to cause the following health effects when people are exposed to it at levels above the MCL for relatively short periods of time: drowsiness, fatigue, headache, and mild eye and respiratory irritation.

Long-term: Ethylbenzene has the potential to cause the following effects from a lifetime exposure at levels above the MCL: damage to the liver, kidneys, central nervous system, and eyes. 


\subsubsection{Toluene}

Short-term: Toluene has the potential to cause the following health effects when people are exposed to it at levels above the MCL for relatively short periods of time: minor nervous system disorders such as fatigue, nausea, weakness, and confusion.

Long-term: Toluene has the potential to cause the following effects from a lifetime exposure at levels above the MCL: more pronounced nervous disorders such as spasms, tremors, impairment of speech, hearing, vision, memory, coordination; and liver and kidney damage.

\subsubsection{Xylenes}

Short-term: Xylenes have the potential to cause the following health effects when people are exposed to it at levels above the MCL for relatively short periods of time: disturbances of cognitive abilities, balance, and coordination.

Long-term: Xylenes have the potential to cause the following effects from a lifetime exposure at levels above the MCL: damage to the central nervous system, liver, and kidneys.

\subsection{Soil Contamination}

Soil samples collected during monitoring well installation on October 19-20, 2005, represent composites based on photoionization detector (PID) readings [3]. Concentrations exceeding North Dakota action levels in soils of 100 and $0.5 \mathrm{mg} / \mathrm{kg}$ for total petroleum hydrocarbons (TPH) and benzene, respectively, were found in all new boreholes located in the south UST area. Maximum values in excess of $4100 \mathrm{mg} / \mathrm{kg}$ for TPH and $39 \mathrm{mg} / \mathrm{kg}$ for benzene indicate the persistent presence of free product within the smear and unsaturated zones (Table 2).

Table 2. BTEX in Soils

\begin{tabular}{|c|c|c|c|c|c|c|}
\hline & & REC-1 & MW-11 & MW-12 & MW-13 & MW-13 \\
\hline Date Sampled & & $11 / 20 / 05$ & $11 / 19 / 05$ & $11 / 19 / 05$ & $11 / 20 / 05$ & $11 / 20 / 05$ \\
\hline Interval (ft) & & 11-13 & 11-13 & $11-13$ & 7-9 & $10-12$ \\
\hline $\mathrm{MTBE}^{1}$ & $\mu \mathrm{g} / \mathrm{kg}$ & $<50$ & $<13.1$ & $<100$ & $<5000$ & $<250$ \\
\hline Benzene & $\mu \mathrm{g} / \mathrm{kg}$ & 1174 & 1186 & 917.1 & 39,260 & 6743 \\
\hline Toluene & $\mu \mathrm{g} / \mathrm{kg}$ & 349 & 113.2 & 183.7 & 184,200 & 711.5 \\
\hline Ethylbenzene & $\mu \mathrm{g} / \mathrm{kg}$ & 484 & 331.9 & 1372 & 83,640 & 6030 \\
\hline Xylenes (Total) & $\mu \mathrm{g} / \mathrm{kg}$ & 1280 & 361 & 1573 & 337,400 & 13,310 \\
\hline $\mathrm{TPH}\left(\mathrm{GRO}^{2}\right)$ & $\mathrm{mg} / \mathrm{kg}$ & 24 & 13 & 77 & 4,100 & 250 \\
\hline
\end{tabular}

\subsection{Groundwater Contamination}

A total of ten groundwater samples, including a sample from municipal well No. 8, and one surface water sample (Cherry Creek) were analyzed for the full suite of volatile organic contaminants (VOCs) using method SW 8021 (465F) (Table 3, Appendix F and G). In addition, 
biodegradation indicators were analyzed in all samples (Appendix F and G-2). Samples were collected using disposable PVC bailers, preserved on-site, and stored on ice. Samples for dissolved metals were filtered using $0.45-\mu \mathrm{m}$ Geotech disposable filters. All analyses were conducted by MVTL in Bismarck, North Dakota, and New Ulm, Minnesota. Quality assurance/quality control (QA/QC) samples totaled one field blank, one trip blank, one equipment blank, and a duplicate. Field-measured parameters including temperature, dissolved oxygen (DO), EC, $\mathrm{pH}$, and ox-redox potential were recorded during sampling. Table 3 and Appendix A provide a summary of analytical results for targeted compounds; complete analytical documentation is in Appendix G. In addition to EERC sampling, COC trends and previous analysis were evaluated based on results of the MPE pilot test [3] and documentation provided by NDDH.

Table 3. COC Concentrations in Groundwater (analyses - December 6, 2006)

\begin{tabular}{|c|c|c|c|c|c|c|c|}
\hline Well ID & $\begin{array}{c}\text { Benzene } \\
\mu \mathrm{g} / \mathrm{l}\end{array}$ & $\begin{array}{c}\text { Toluene } \\
\mu \mathrm{g} / \mathrm{l}\end{array}$ & $\begin{array}{c}\text { Ethylbenzene } \\
\mu \mathrm{g} / \mathrm{l}\end{array}$ & $\begin{array}{l}\text { Xylenes } \\
\text { (total) } \mu g / l\end{array}$ & $\begin{array}{c}\text { GRO (TPH) } \\
\mathrm{mg} / \mathrm{l}\end{array}$ & $\begin{array}{c}\text { DCA }^{1} \\
\mu g / l\end{array}$ & $\begin{array}{c}\text { TMB }^{2} \\
\mu \mathrm{g} / \mathrm{l}\end{array}$ \\
\hline MW-1 & $<0.5$ & $<0.6$ & $<0.6$ & $<3$ & $<0.2$ & $<0.5$ & $<0.7$ \\
\hline MW-5 & $<0.5$ & $<0.6$ & $<0.6$ & $<3$ & $<0.2$ & $<0.5$ & $<0.7$ \\
\hline MW-6 & 39.9 & $<0.6$ & 7.1 & 4.4 & $<0.2$ & 1.6 & $<0.7$ \\
\hline MW-8 & $<0.5$ & $<0.6$ & $<0.6$ & $<3$ & $<0.2$ & $<0.5$ & $<0.7$ \\
\hline MW-9 & $<0.5$ & $<0.6$ & $<0.6$ & $<3$ & $<0.2$ & $<0.5$ & $<0.7$ \\
\hline MW-11 & 18790 & 1008 & 2125 & 2534 & 53.7 & 2466 & 1232 \\
\hline MW-13 & 29370 & 4430 & 3814 & 9538 & 112.7 & 1828 & 1674 \\
\hline REC-1 & 17800 & 862.9 & 3206 & 4054 & 65.63 & 2095 & 1316 \\
\hline Cherry Cr. & $<0.5$ & $<0.6$ & $<0.6$ & $<3$ & $<0.2$ & $<0.5$ & $<0.7$ \\
\hline City Well 8 & $<0.5$ & $<0.6$ & $<0.6$ & $<3$ & $<0.2$ & $<0.5$ & $<0.7$ \\
\hline EPA MCL & 5 & 1000 & 700 & 10000 & 0.5 & 5 & \\
\hline$N D D H^{3}$ & 5 & 5 & 5 & 5 & & & \\
\hline
\end{tabular}

Free product was detected in MW-4 $(0.2 \mathrm{ft})$ and MW-12 $(0.1 \mathrm{ft})$. Concentrations of target contaminants considerably exceed EPA MCL regulatory levels and North Dakota action levels for benzene in monitoring wells MW-6, 11, 13 and recovery well REC-1 (Table 3). In addition, the presence of 1,2,4-trimethylbenzene (of gasoline origin) and the 1,2-dichloroethane as high as $2466 \mu \mathrm{g} / \mathrm{l}$ was detected at the site.

\subsection{Hydraulic Properties and Contaminant Transport}

Hydraulic testing was conducted in December 2006 and as an integral part of the MPE pilot test in April 2006. A slug test was conducted on well REC-1 to evaluate hydraulic parameters. Interpretation of the hydraulic test is summarized in Appendix D. The slug consisted of a 3-inch-diameter PVC pipe which was filled with sand and sealed at both ends. The volume displaced by the slug was 1.0 gallon $(3.785 \mathrm{~L})$. Aquifer response was recorded using a Telog ${ }^{\circledR}$ PR-31 data collection system, with standard manual water level (water level indicator) collection for confirmation. 
Hydraulic conductivity was $6 \times 10^{-5} \mathrm{ft} / \mathrm{min}\left(3 \times 10^{-7} \mathrm{~m} / \mathrm{s}\right)$ using the Bower-Rice method and $9 \times 10^{-5} \mathrm{ft} / \mathrm{min}\left(5 \times 10^{-7} \mathrm{~m} / \mathrm{s}\right)$ using the Hvorslev algorithm for slug test. Extremely low values of hydraulic conductivity and high vacuum required to overcome formation resistance to hydraulic and pneumatic flow, including low yield (0.07-0.3 gpm) documented from the MPE pilot test, indicate extremely low potential for off-site contaminant transport.

The calculated values are representative of horizontal hydraulic conductivity. Reflecting on heterogeneous site geology and considerable presence of a clayey fraction in the upper portion of the sediment profile, the vertical conductivity is typically several orders of magnitude smaller. In order to account for the worst-case scenario and dual porosity (matrix versus preferential flow), an aquifer saturated thickness of $0.5-1.5 \mathrm{ft}$ was used for interpretation to account for expected preferential flow in more permeable layers/lenses. The difference of about $10 \%$ in calculated values for hydraulic conductivity was negligible. An additional factor contributing to reduction of $\mathrm{COC}$ migration downgradient is the confined nature of the underlying aquifer, with gradient toward the surface.

The contaminated area lies close to the wellhead protection area for the northern municipal well field (Appendix E) but not in the capture zone. With respect to the results of this project, construction of the municipal well, and the results of detailed capture zone analysis, including well head delineation conducted by NDDH [10], the potential for contaminant migration to the municipal well field is extremely limited.

\subsection{Municipal Well - Capture Zone Analysis}

Detailed analysis of the capture zone and well head protection area (WHPA) for the municipal well field was completed by NDDH in 2006 [10]. The WHPA delineation presented in Appendix $E$ is based on the zone of contribution method using a modular, semianalytical groundwater flow model developed by EPA. The current WHPA was delineated using hydraulic properties of the Tobacco Garden Creek Aquifer and is based on a 10-year time of travel under constant pumping condition. In the absence of site-specific hydraulic information and limited capabilities of the EPA model, the selected approach represents a reasonably conservative scenario. With respect to extreme heterogeneity and random occurrence of preferential flow pathways (layers, lenses) in sediments overlying the Tobacco Garden Creek Aquifer, the uncertainties associated with definition of leakage and transport parameters would outweigh the benefits and reliability of the numerical modeling. The primary factors supporting adequate aquifer protection as defined by the current WHPA are as follows:

- Hydraulic conductivity of the Tobacco Garden Creek Aquifer (150-200 ft/day) is several orders of magnitude higher that those for overlying sediments $(0.1 \mathrm{ft} / \mathrm{day})$.

- The Tobacco Garden Creek Aquifer is confined, with a static water table at about $7 \mathrm{ft}$ below the surface at well No. 8 [10]. The water table at the site is at 10-13 ft belowground.

- Groundwater flow direction in the deeper, Tobacco Garden Creek Aquifer, is to the north, north-east-north, i.e., COC-impacted site is downgradient from municipal wells.

- The model is using a 10-year time of travel under constant pumping conditions. Actual aquifer usage is based on a demand-driven intermittent pumping. 


\subsection{Exposure Factors}

The primary exposure factors were evaluated in accordance with ASTM International's Standard Guide for Risk-Based Corrective Action [11]. The exposure assessment is based on evaluation of the magnitude, frequency, duration, and route of exposure between a source area and a receptor.

\subsubsection{Exposure Pathways}

The contaminated soil and groundwater is several feet below the ground surface. Unless disturbed (excavation), there is no identifiable direct exposure pathway at the source. The only realistic exposure pathway is indirect via exposure to contaminated groundwater after its migration off-site. With respect to site-specific geotechnical conditions described in detail in previous sections, the COC off-site migration and its leakage to the underlying confined aquifer is extremely limited, with the potentially impacted area outside the zone of groundwater use. In addition, the hydraulic factors (gradient, flow direction, confined nature) and construction of the municipal well further limits potential for COC to enter the water supply chain. The municipal well No. 8 closest to the site $(\sim 700 \mathrm{ft})$, intercepts the source aquifer at $51 \mathrm{ft}$. The well is cased with stainless steel to $78 \mathrm{ft}$, grouted to $60 \mathrm{ft}$, and screened from 78-108 ft.

\subsubsection{Exposure Routes}

In the absence of a direct exposure pathway and the low probability of an indirect exposure pathway, water intake by ingestion is the only realistic scenario for the exposure route. Providing the site is excavated (corrective action, construction, etc.), additional exposure routes would be inhalation of VOCs and dermal contact with contaminated soil.

\subsubsection{Receptors}

The site is located in an industrial zone. Occupational hazards are minimal because of intercepted exposure pathways (below the surface location of impacted soils and groundwater) and open field area. In case of excavation of contaminated soils, a short-term exposure to volatile contaminants would have to be addressed by proper material handling and appropriate personal protective equipment. With respect to high vapor pressure (high volatilization rates for primary carcinogens), the potential exposure would be short term. There is no residential exposure or receptors at or downgradient from the impacted area.

\subsection{CONCLUSIONS AND RECOMMENDATIONS}

The risk analysis presented is based on compilation of information from the site-specific geotechnical investigation, evaluation of contaminant properties, a receptor survey, capture zone analysis, and evaluation of the wellhead protection area for the municipal well field. The project results indicate that the risks associated with contaminant occurrence at the Construction Services, Inc., site are low and, under current conditions, there is no direct or indirect exposure pathway between the contaminated groundwater and soils and potential receptors. follows:

The results of the investigation and primary factors contributing to risk reduction are as 
- Expanded probing activity to tank areas west and north of the facility did not confirm the presence of the free product. The area of impacted soil and groundwater is limited to the tank area southwest of the facility.

- Based on the geological profile and results the LIF and hydraulic testing, residual free product is retained in thin discrete layers or lenses of sandy silt and silty sand within the smear zone of fluctuating groundwater.

- Low values of hydraulic conductivity, high formation resistance to hydraulic and pneumatic flow, and low yield indicate extremely low potential for off-site contaminant transport.

- The hydraulic conductivity of the underlying Tobacco Garden Creek Aquifer is several orders of magnitude higher than that for overlying sediments. In combination with confined aquifer conditions, it minimizes potential for vertical migration to deeper waterbearing sediments.

- Groundwater flow direction in the deeper Tobacco Garden Creek Aquifer is to the north, north-east-north, i.e., the COC-impacted site is downgradient from municipal wells.

- The impacted area does not lie in the capture zone for the municipal well field and is outside of the well head protection area as defined by NDDH.

- There is no direct exposure pathway between the contaminant source and the potential receptor at this time.

- There is no residential exposure or receptors at or downgradient from the impacted area.

It is important to note that site-specific conditions and risk-limiting factors never completely eliminate the risks associated with contaminant occurrence. The site-specific geotechnical conditions considerably limit options for remedial alternatives. The MPE pilot test confirmed the applicability of vacuum enhancement; however, the technology would have to be modified to overcome limitations associated with tight geology and would be more expensive than excavation. In the case where risk elimination is required, the excavation of the impacted area and replacement with inert, well-compacted soil is identified as the economically most feasible option.

Based on the results of the risk analysis presented, it is recommended to:

- Seal and abandon all wells at the site with the exception of wells MW-5, MW-8, and MW-9.

- Initiate biannual water quality monitoring in wells MW-5, MW-8, and MW-9 for target COC, namely BTEX and 1,2 dichloroethane.

- Periodically sample municipal well No. 8 with analyses for BTEX and 1,2dichloroethane. 


\subsection{REFERENCES}

1. Preliminary Subsurface Assessment Report, Former Chinook Pipeline Property Watford City, North Dakota. Braun Intertec Environmental, Inc., 1992.

2. Corrective Action Plan to Mr. Kevin DeTienne, Former Chinook Pipeline Property Watford City, North Dakota. Braun Intertec Environmental, Inc., 1993.

3. Solc J.; Botnen, B.W., 2006, Feasibility of Accelerated Risk Reduction Using Innovative Vacuum-Enhanced Plume Controls, Watford City, North Dakota; Final Report for North Dakota Department of Health; EERC Publication 2006-EERC-06-04; Grand Forks, ND.

4. North Dakota State Water Commission. www.swc.state.nd.us (accessed 2007).

5. U.S. Environmental Protection Agency www.epa.gov/ogwdw/dwh/c-voc/12-dichl.html (accessed 2007).

6. The Merck Index. 12th Edition; Merck \& Co., Inc.: Whitehouse Station, NJ, 1996.

7. Suthersan, S. Natural and Enhanced Remediation Systems. Arcadis - Geraghty \& Miller Environmental Science and Engineering Series, 2001.

8. Nyer, E. In Situ Treatment Technologies Second Edition, Arcadis - Geraghty \& Miller Environmental Science and Engineering Series, 2000.

9. Montgomery, J.H., Welkom, L.M. Groundwater Chemicals Desk Reference. Lewis Publishers, 1990.

10. City of Watford City, McKenzie County, ND - Wellhead Protection Area Delineation, North Dakota Wellhead Protection Program, North Dakota Department of Health - Division of Water Quality, Oct 2006.

11. ASTM Standard Guide for Risk-Based Corrective Action. ASTM International, E 2081-00, Nov 2000. 
APPENDIX A

SITE PLAN 


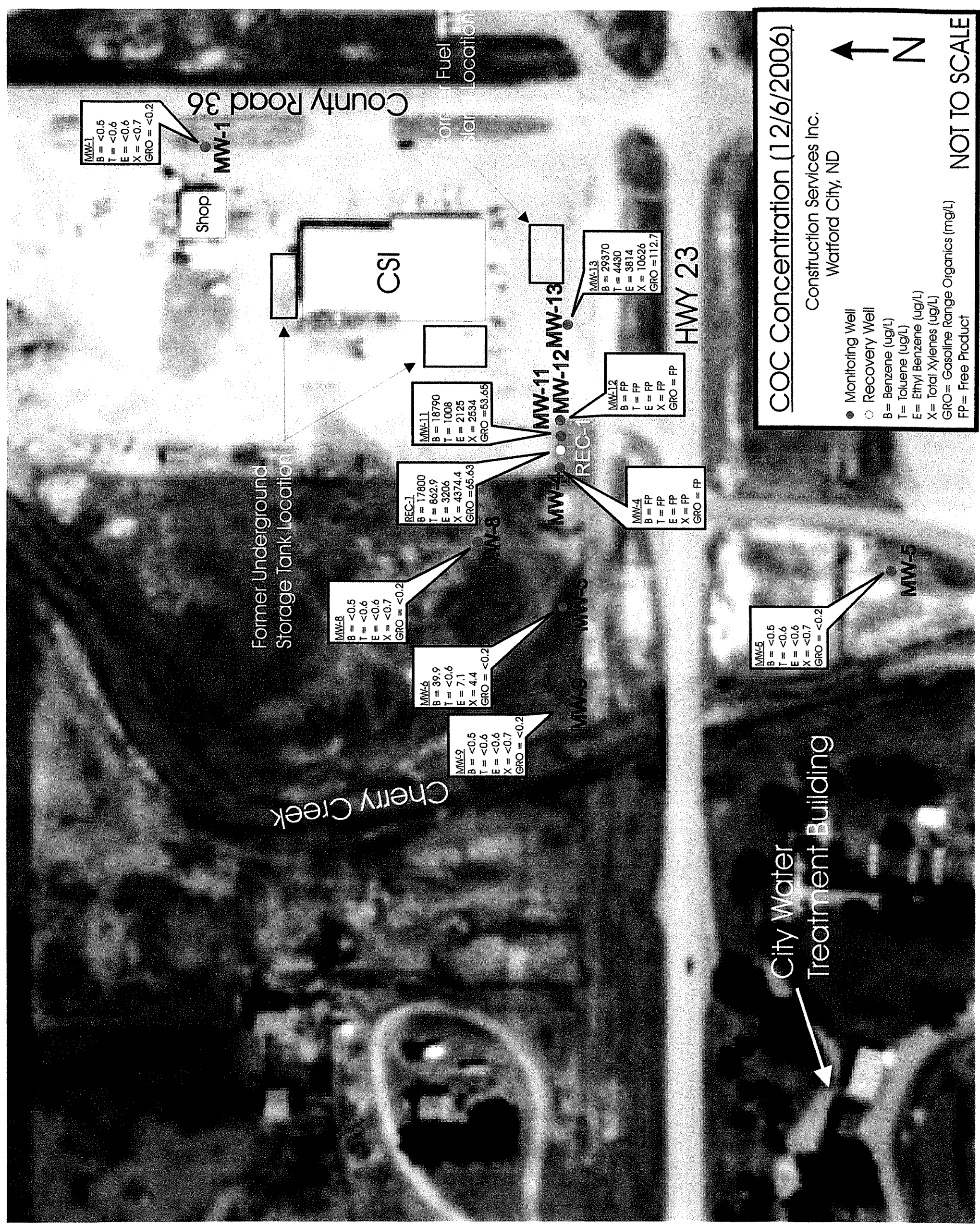


APPENDIX B

LIF DOCUMENTATION 


\section{E pDod Kłunos}
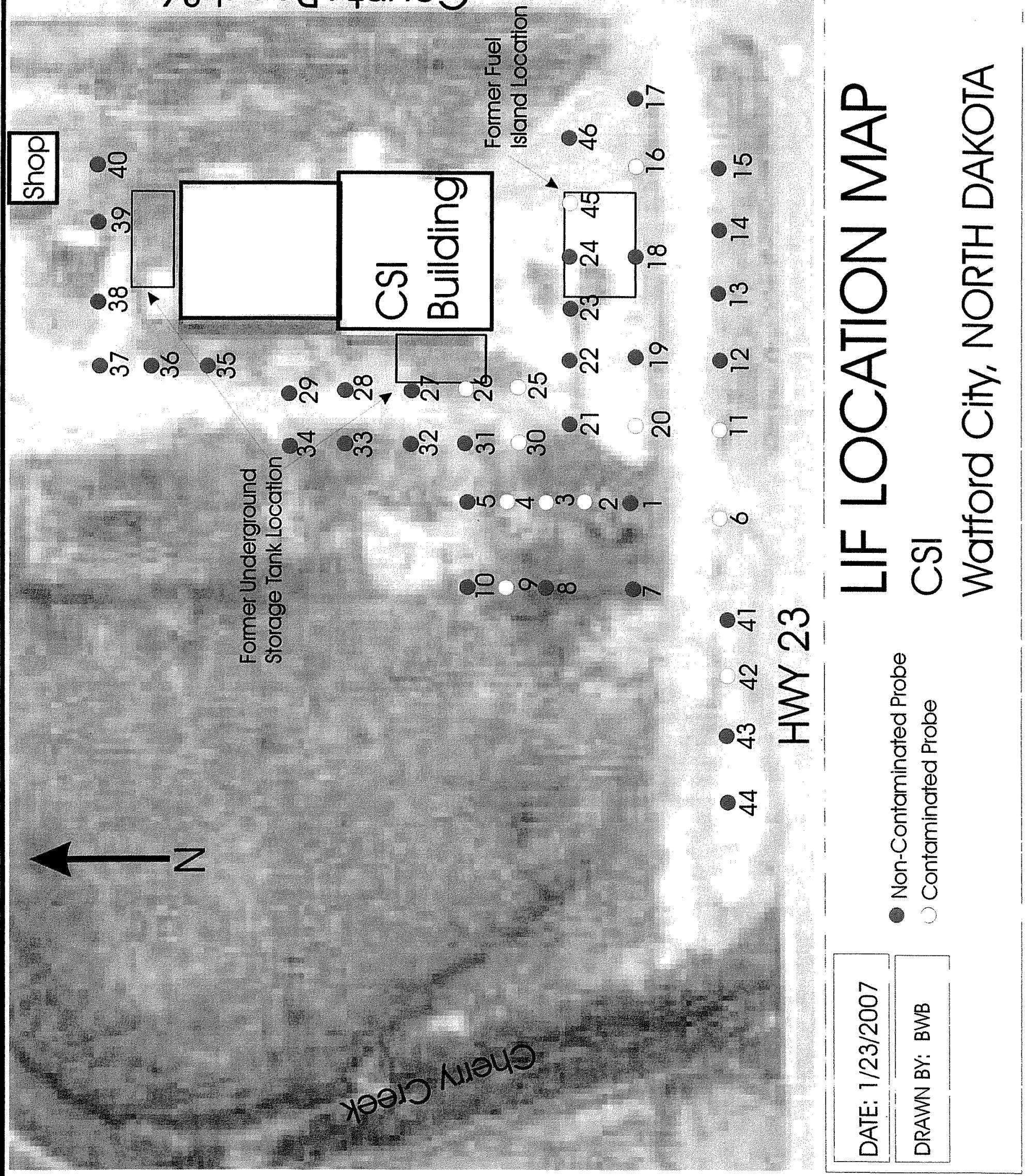
Site: CSI Watford City, ND

Client: EERC

Date/Time: 11/9/2005 @ 4:01:36 PM

ROST Unit: DTI-01
Operator: Steve Adamek

GPS Fix:

Max fluorescence: $0.81 \% @ 0.52 \mathrm{ft}$

Final depth BGS: $17.89 \mathrm{ft}$

\section{WCLIF01}

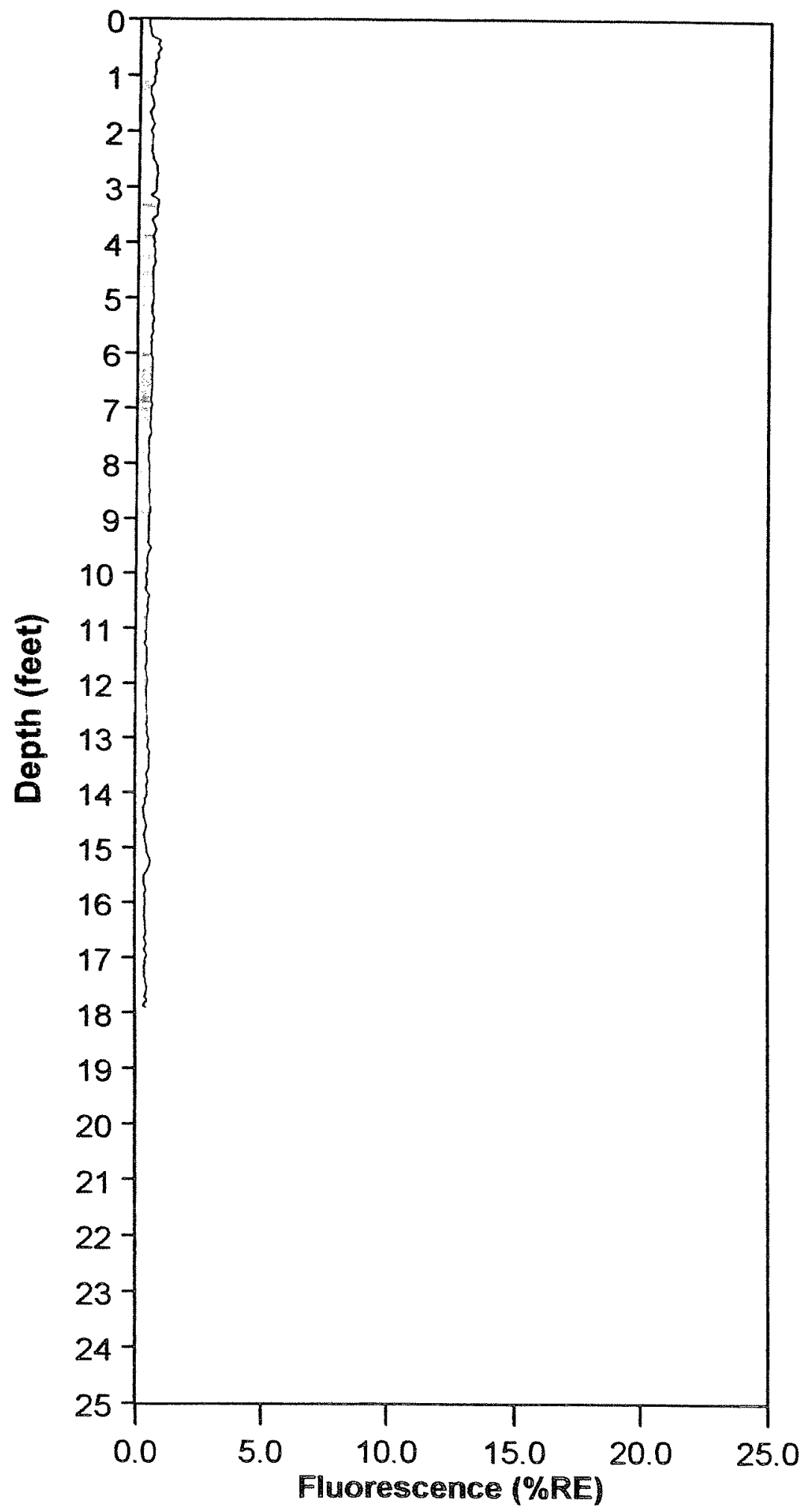

Dakota Technologies, Inc., 2201-A 12th St N., Fargo, ND 58102 (701) 237-4908 whw.dakotatechnologies.com 
ROST Fluorescence Response Data

\begin{tabular}{|l|l|}
\hline Site: CSI Watford City. ND & Operator: Steve Adamek \\
Client: EERC & GPS FIx: \\
DaterTime: $11 / 9 / 2005 @ 4: 24: 24$ PM & Max fluorescence: $100.58 \% @ 9.64 \mathrm{ft}$ \\
ROST Unit: DTI-01 & Final depth BGS: $22.00 \mathrm{ft}$ \\
\hline
\end{tabular}

WCLIF02

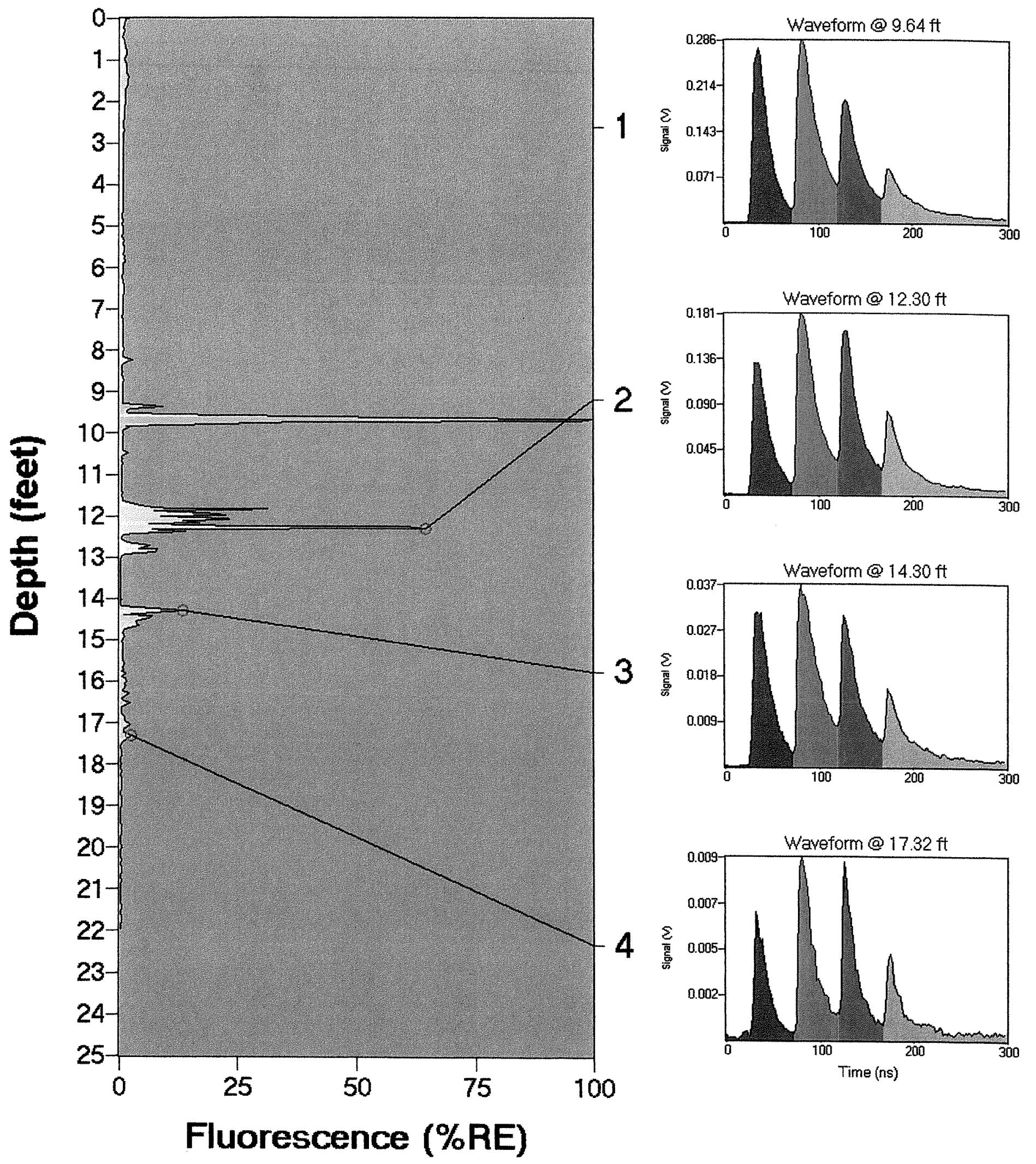

Dakota Technologies. Inc., 2201-A 12th St N.. Fargo. ND 58102 (701) 237-4908 www.dakotatechnologies.com 
ROST Fluorescence Response Data

\begin{tabular}{|l|l|}
\hline Site: CSI Watford City. ND & Operator: Steve Adamek \\
Client: EERC & GPS FIX: \\
DaterTime: 11/912005@ 4:47:06 PM & Max fluorescence: $120.76 \% @ 14.20 \mathrm{ft}$ \\
ROST Unit: DTI-01 & Final depth BGS: $17.90 \mathrm{ft}$ \\
\hline
\end{tabular}

\section{WCLIF03}

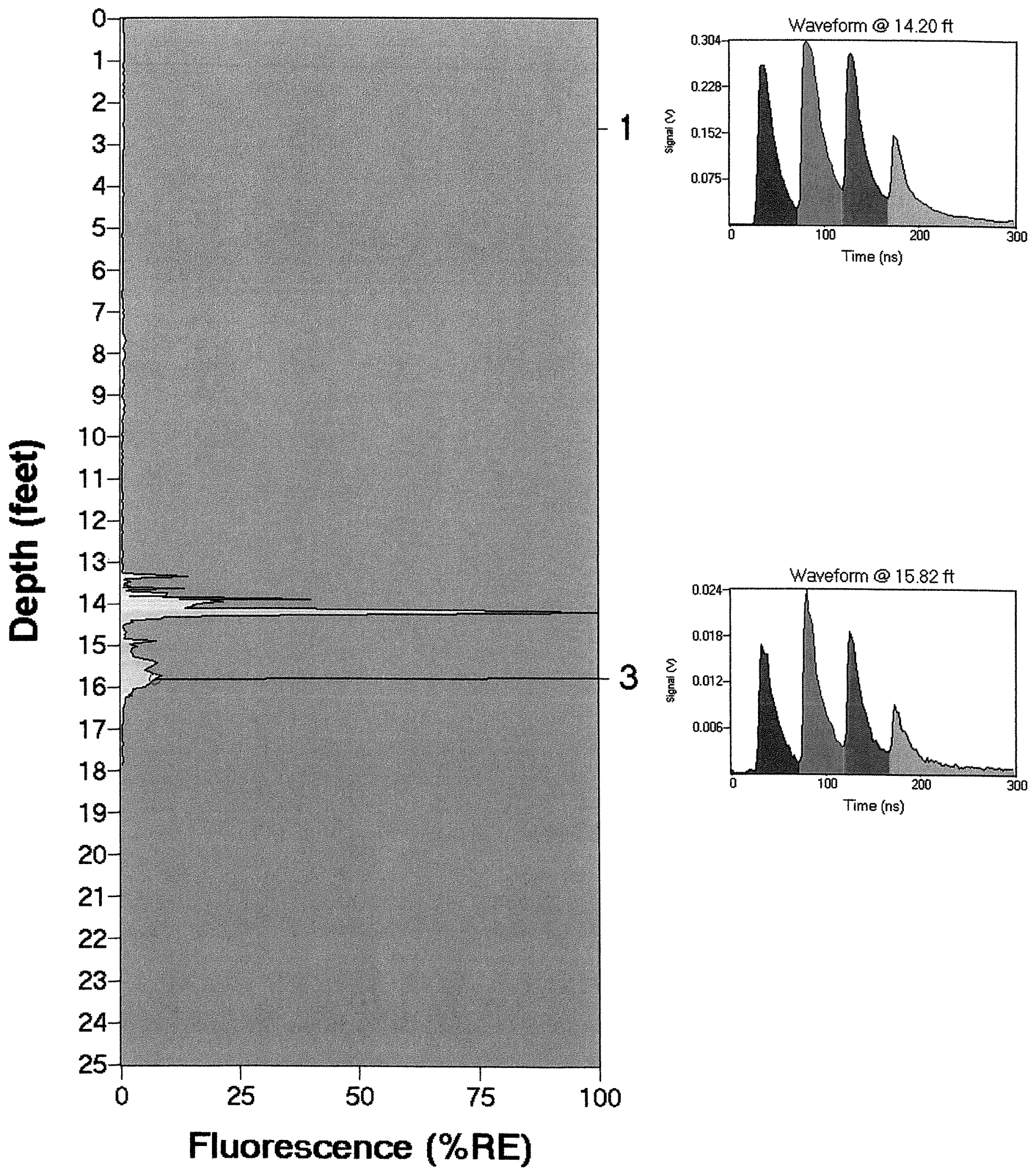




\begin{tabular}{|l|l|}
\hline Site: CSI Watford City, ND & Operator: Steve Adamek \\
Client: EERC & GPS Fix: \\
Date/Time: $11 / 9 / 2005 @ 5: 06.01$ PM & Max fluorescence: $1.82 \% @ 11.68 \mathrm{ft}$ \\
ROST Unit: DTI-01 & Final depth BGS: $18.02 \mathrm{ft}$ \\
\hline
\end{tabular}

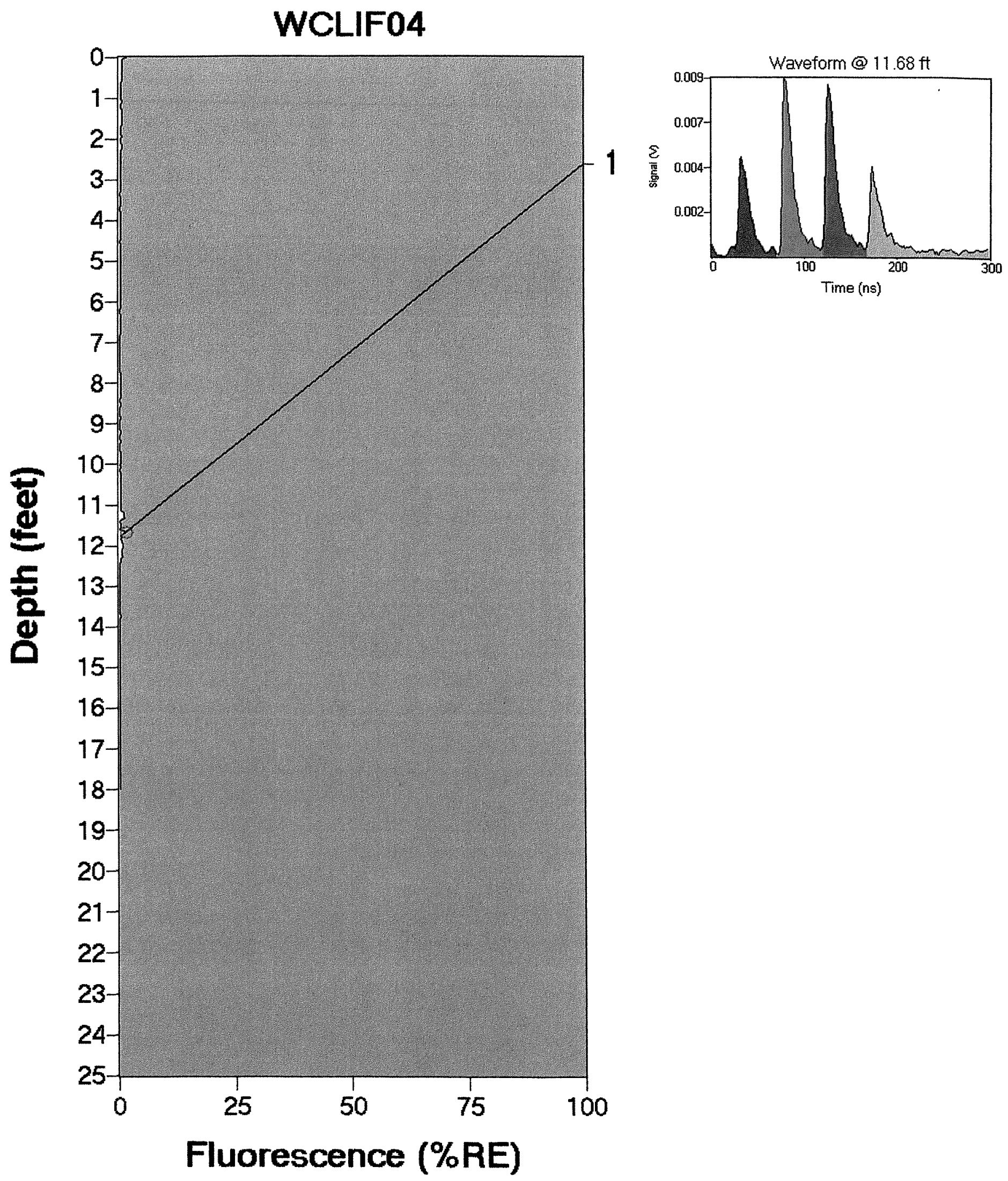

Dakota Technologies, Inc., 2201-A 12th St N. Fargo. ND 58102 (701) 237-4908 wm.dakotatechnologies.com 


\section{ROST Fluorescence Response Data}

\begin{tabular}{l|l} 
Site: CSI Watford City, ND & Operator: Steve Adamek \\
Client: EERC & GPS Fix: \\
Date/Time: $11 / 9 / 2005 @ 5: 21: 42$ PM & Max fluorescence: $1.01 \% @ 0.22 \mathrm{ft}$ \\
ROST Unit: DTI-01 & Final depth BGS: $17.98 \mathrm{ft}$
\end{tabular}

\section{WCLIF05}

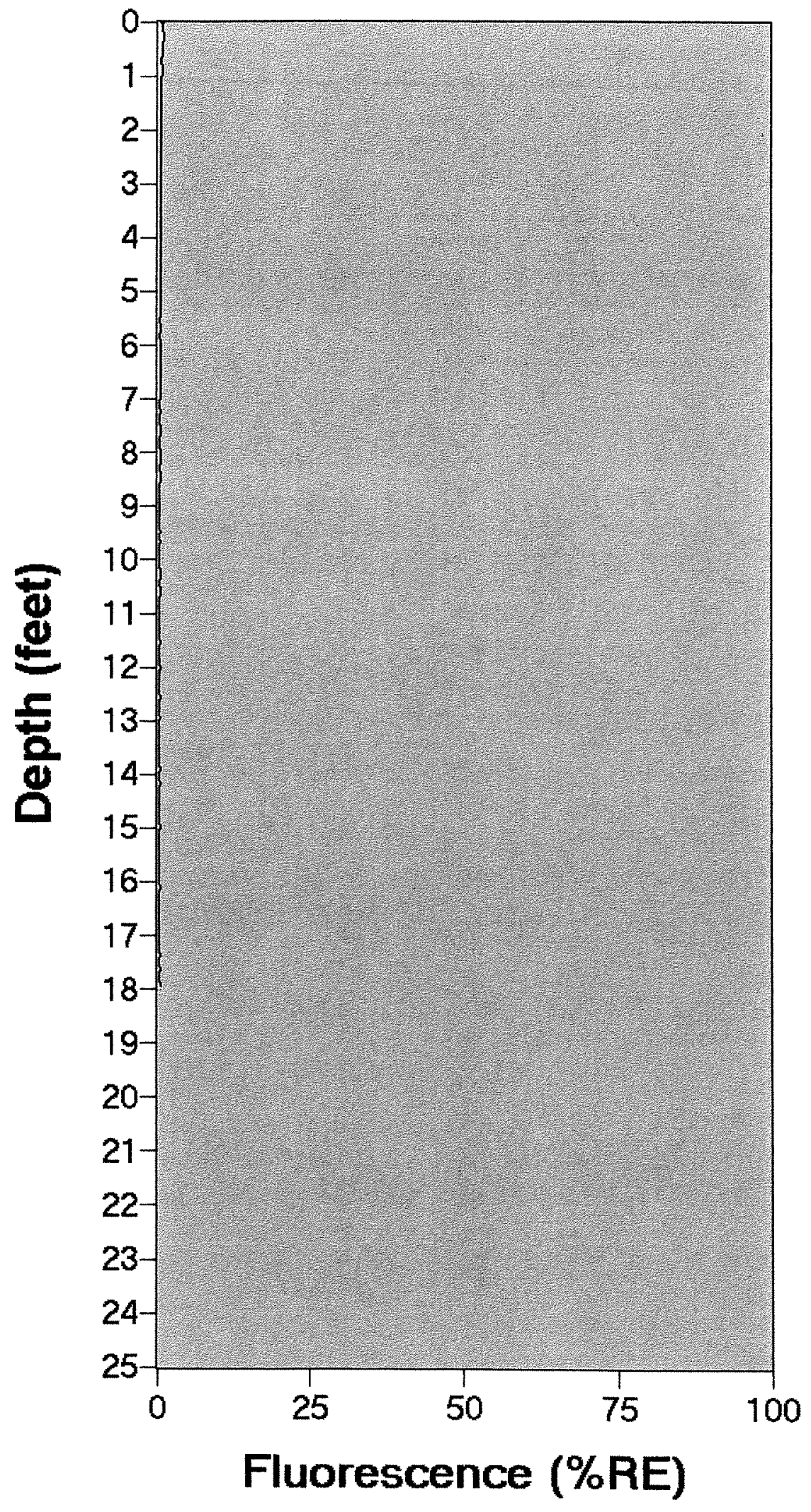

Dakota Technologies. Inc. 2201-A 12th St N. Fargo. ND 58102 (701) 237-4908 www.dakotatechnologies.com 
ROST Fluorescence Response Data

Site: CSI Watford City. ND

Client: EERC

Date/Time:11/9/2005@ 5:37:50 PM

ROST Unit: DTI-01
Operator: Steve Adamek

GPS Fix:

Max fluorescence: $23.49 \% @ 13.39 \mathrm{ft}$

Final depth BGS: $18.02 \mathrm{ft}$

\section{WCLIF06}

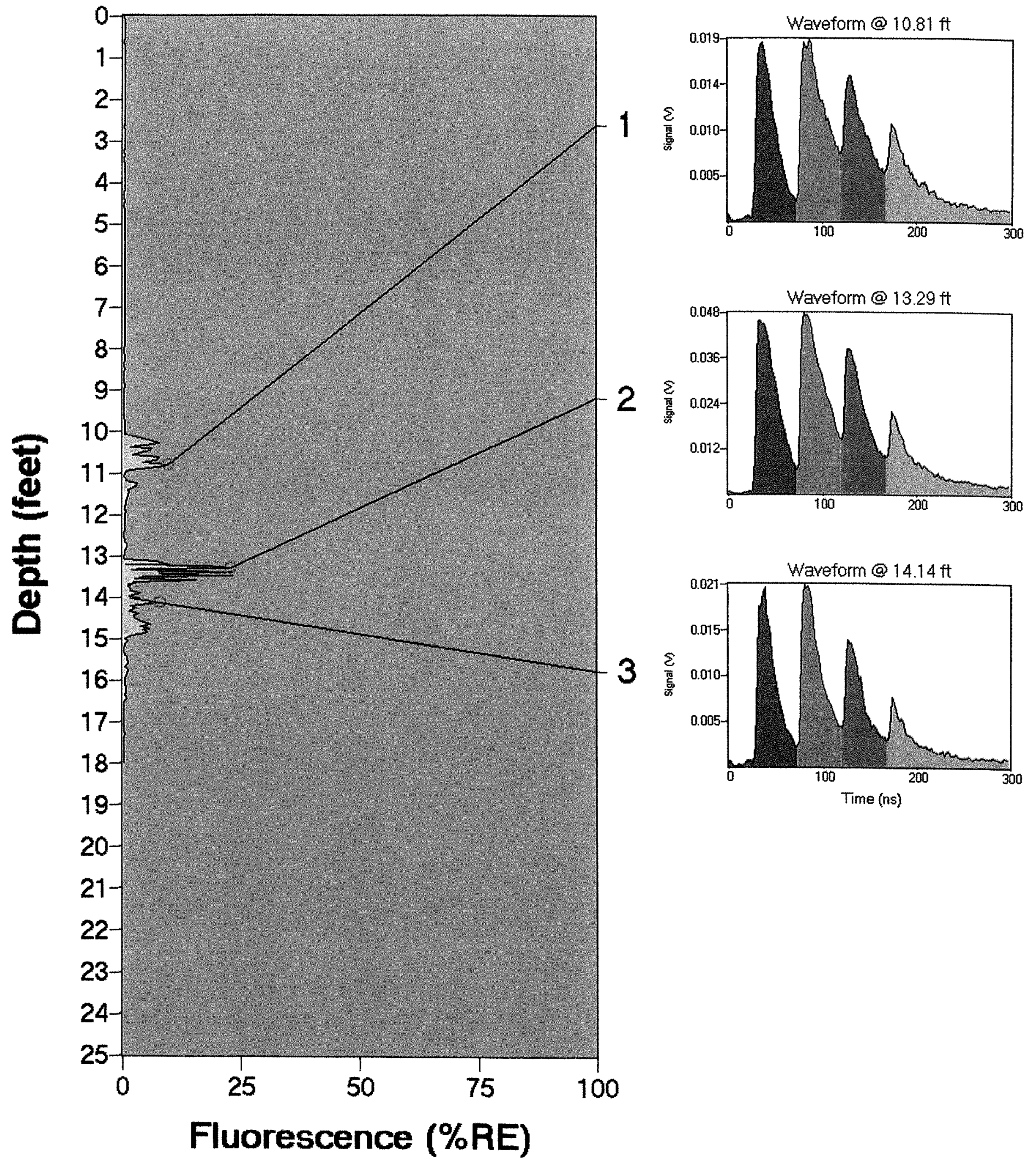


ROST Fluorescence Response Data

Site: CSI Watford City, ND

\section{Client: EERC}

DaterTime: 11/9/2005@ 5:55:58 PM

ROST Unit: DTI-01
Operator: Steve Adamek

GPS FIX:

Max fluorescence: $13.03 \% @ 12.40 \mathrm{ft}$

Final depth BGS: $17.93 \mathrm{ft}$

\section{WCLIF07}

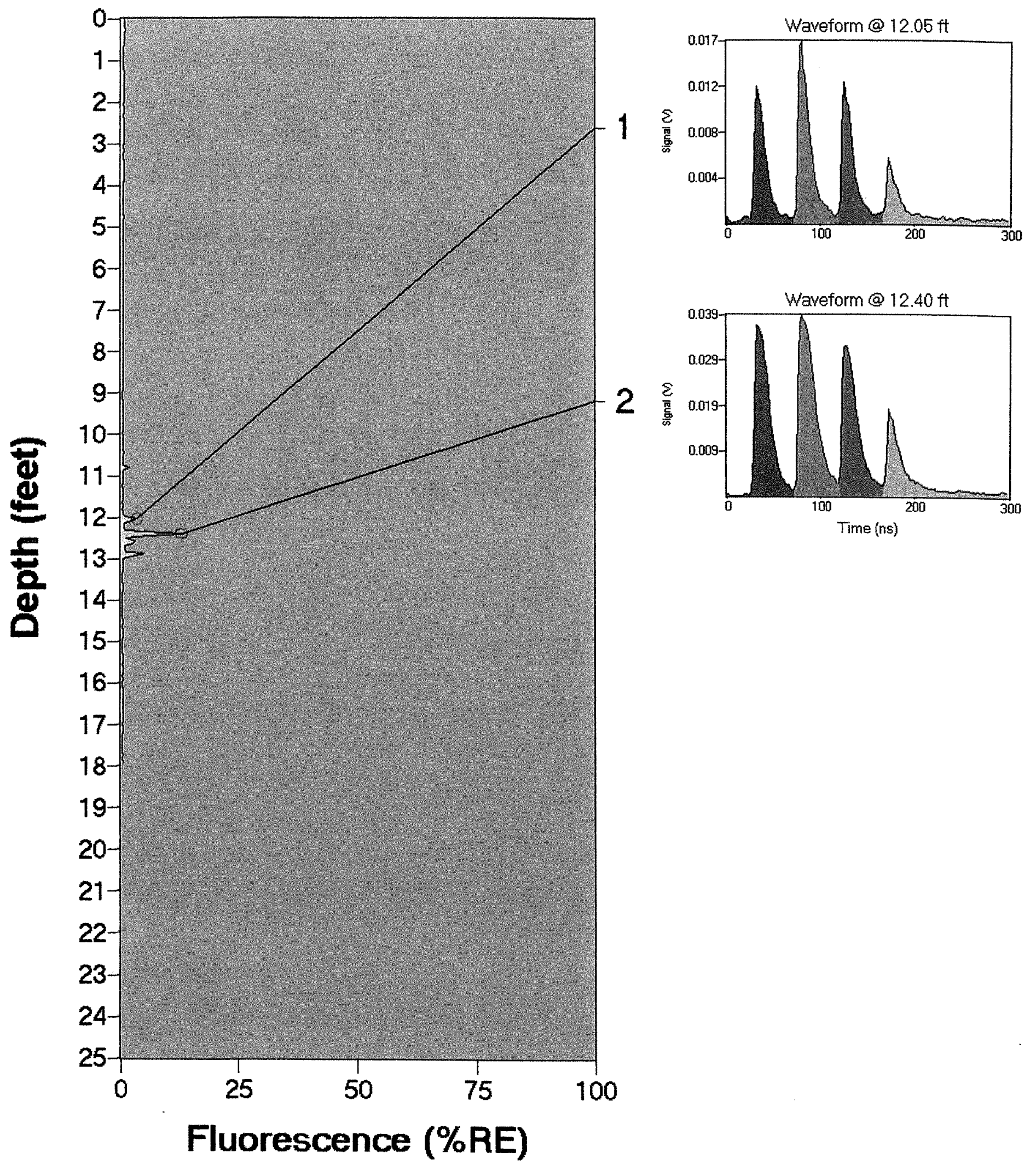


ROST Fluorescence Response Data

\begin{tabular}{l|l} 
Site: CSI Watford City, ND & Operator: Steve Adamek \\
Client: EERC & GPS Fix: \\
DateTime: 11/9/2005@6:12:22 PM & Max fluorescence: $1.43 \% @ 9.33 \mathrm{ft}$ \\
ROST Unit: DTI-01 & Final depth BGS: $17.97 \mathrm{ft}$
\end{tabular}

\section{WCLIF08}

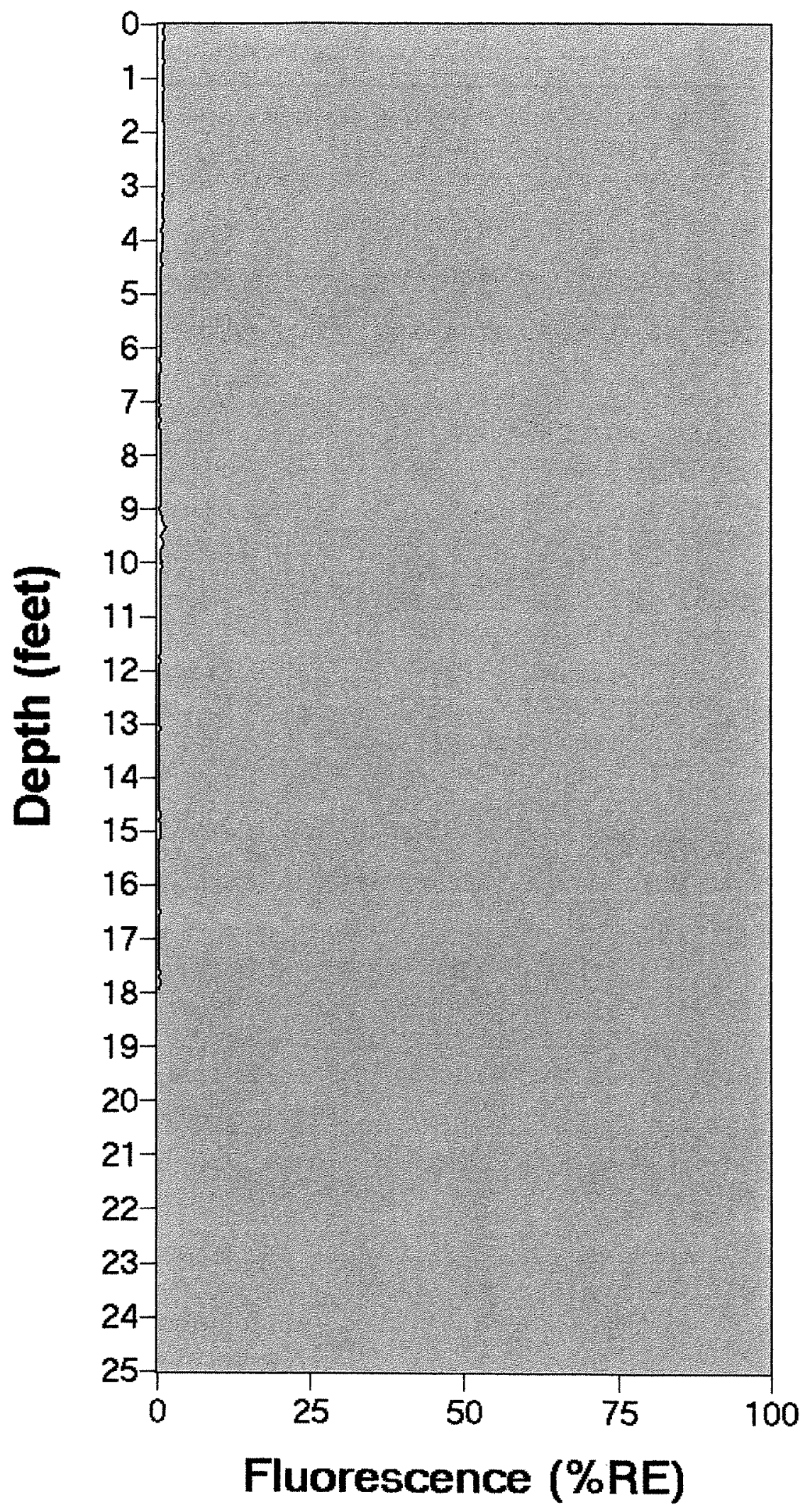

Dakota Technologies, Inc. 2201-A 12th St N.. Fargo. ND 58102 (701) 237-4908 www dakotatechnologies.com 
ROST Fluorescence Response Data

\begin{tabular}{|l|l|}
\hline Site: CSI Watford City, ND & Operator: Steve Adamek \\
Client: EERC & GPS Fix: \\
Date/Time: $11 / 9 / 2005 @ 6: 34: 14$ PM & Max fluorescence: 16.46\%@11.34 ft \\
ROST Unit: DTI-01 & Final depth BGS: 17.87ft \\
\hline
\end{tabular}

\section{WCLIF09}

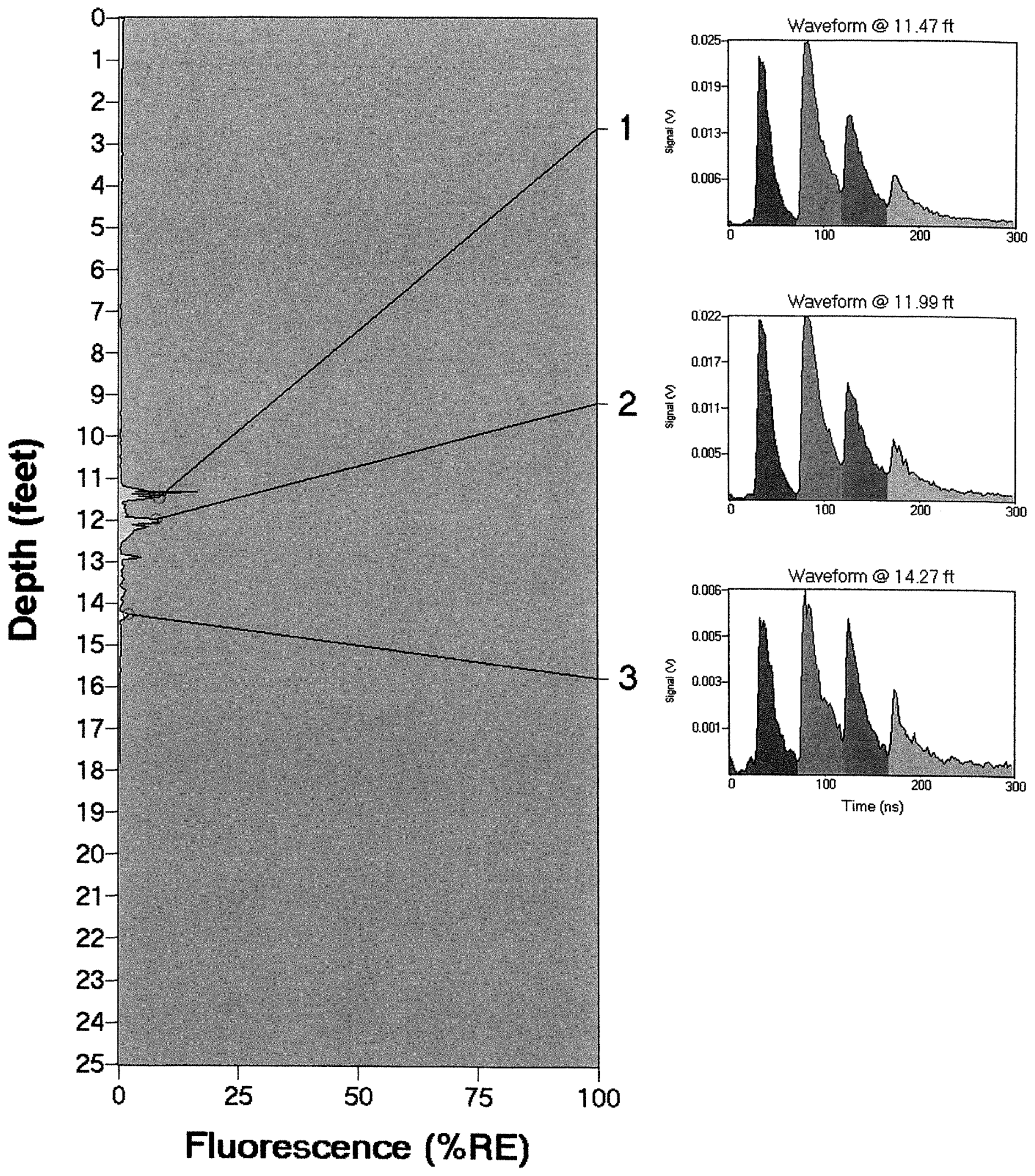


ROST Fluorescence Response Data

\begin{tabular}{l|l} 
Site: CSI Watford City, ND & Operator: Steve Adamek \\
Client: EERC & GPS FIx: \\
Date/Time: 11/10/2005@8:26:02 AM & Max fluorescence: 0.96\%@0.45 ft \\
ROST Unit: DTI-01 & Final depth BGS: $18.02 \mathrm{ft}$
\end{tabular}

WCLIF10

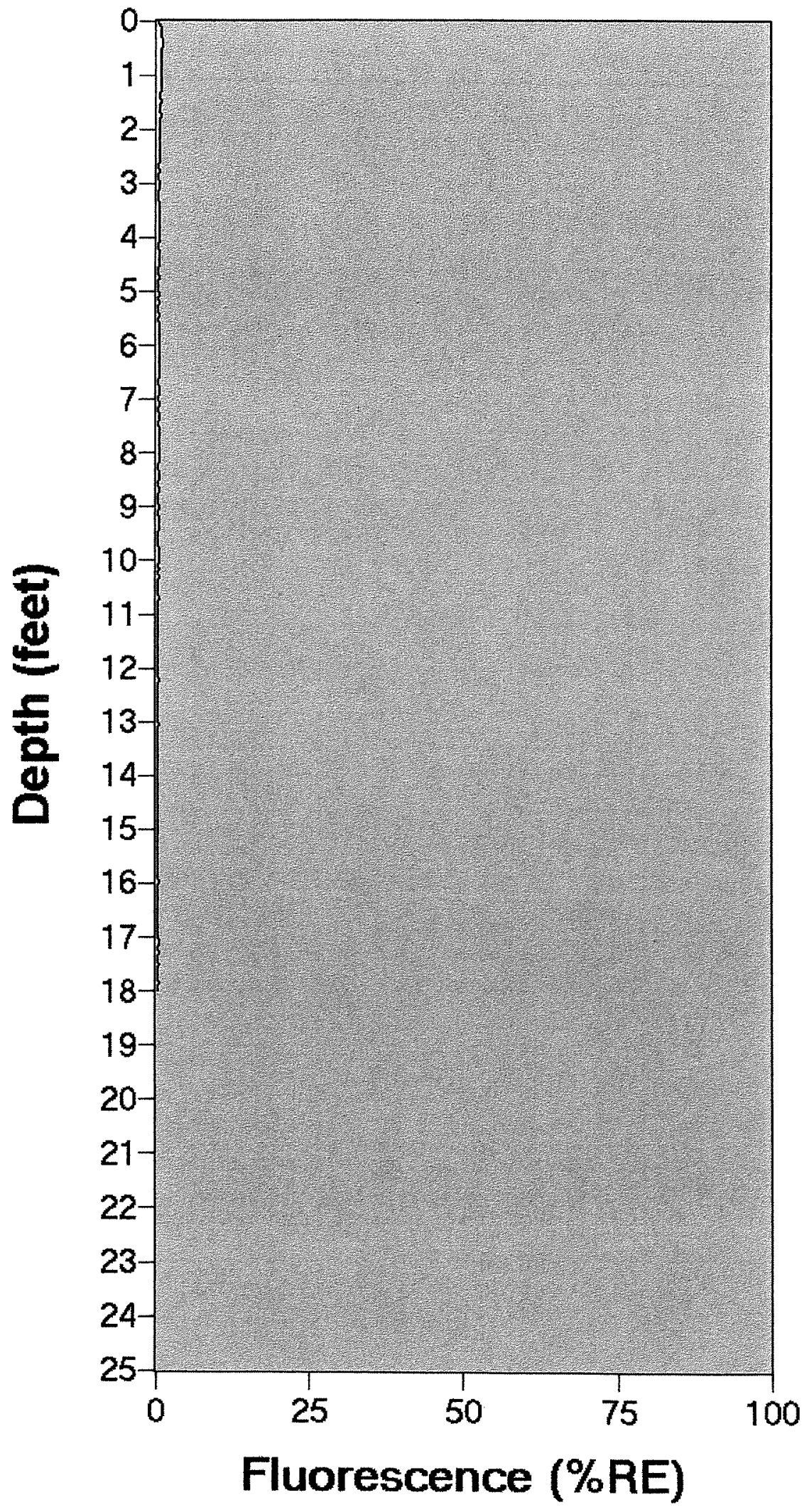

Dakota Technologies. Inc. 2201-A 12th St N. Fargo. ND 58102 (701) 237-4908 www.dakotatechnologies,com 
ROST Fluorescence Response Data

Site: CSI Watford City. ND

Client: EERC

Date/Time: 11/10/2005@8:47:32 AM

ROST Unit: DTI-01
Operator: Steve Adamek

GPS Fix:

Max fluorescence: $37.33 \% @ 13.44 \mathrm{ft}$

Final depth BGS: $20.00 \mathrm{ft}$

\section{WCLIF11}

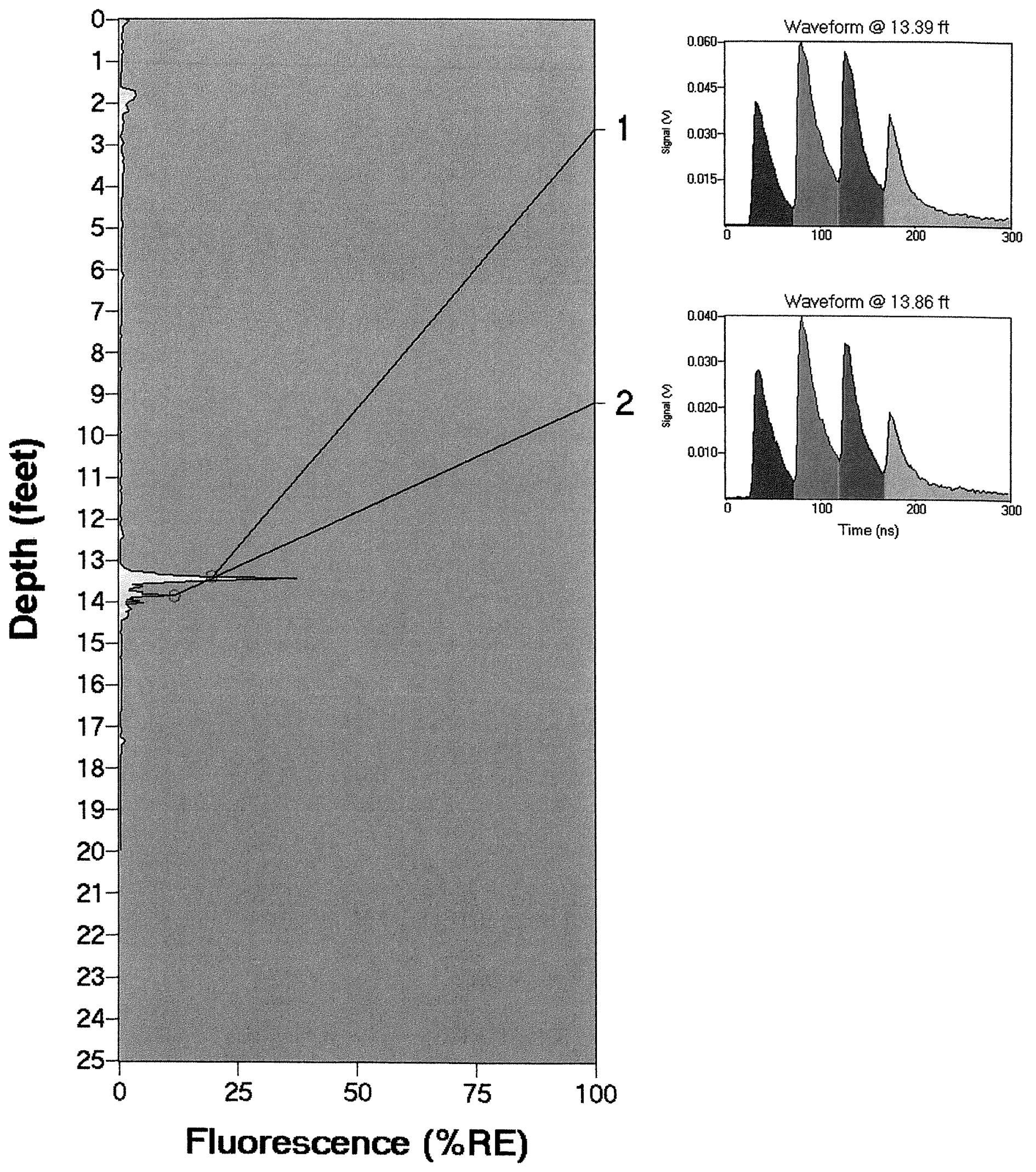


ROST Fluorescence Response Data

\begin{tabular}{|l|l|}
\hline Site: CSI Watford City. ND & Operator: Steve Adamek \\
Client: EERC & GPS Fix: \\
Date/Time: $11 / 10 / 2005 @ 9: 11: 43$ AM & Max fluorescence: $1.38 \% @ 0.54 \mathrm{ft}$ \\
ROST Unit: DTI-01 & Final depth BGS: $18.00 \mathrm{ft}$ \\
\hline
\end{tabular}

\section{WCLIF12}

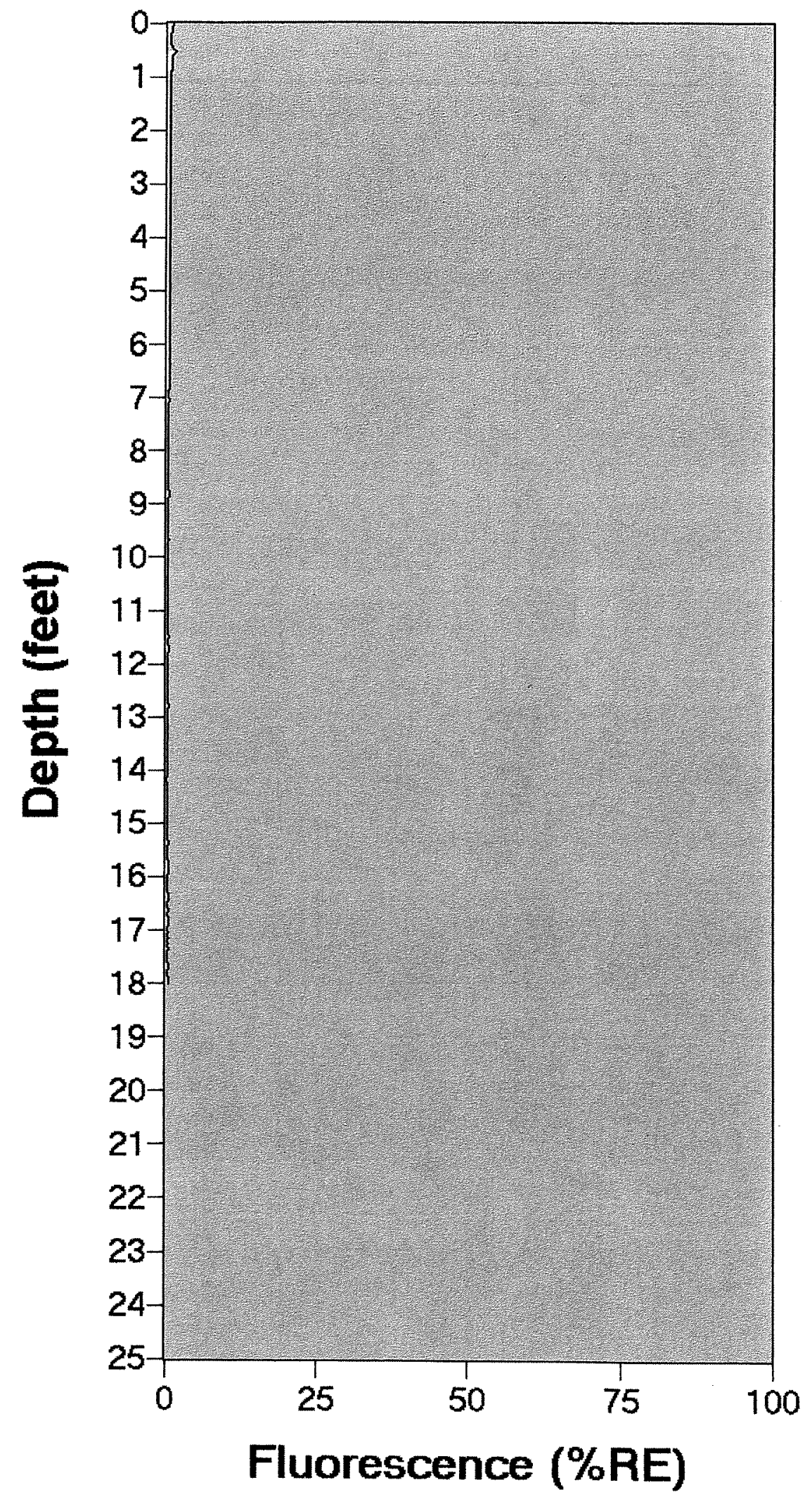

Dakota Technologies. Inc. 2201-A 12th St N. Fargo. ND 58102 (701) 237-4908 whw dakotatechnologies.com 
ROST Fluorescence Response Data

\begin{tabular}{|l|l|}
\hline Site: CSI Watford City. ND & Operator: Steve Adamek \\
Client: EERC & GPS FIX: \\
Date/Time: $11 / 10 / 2005 @ 9: 29: 45$ AM & Max fluorescence: $2.96 \% @ 0.00 \mathrm{ft}$ \\
ROST Unit: DTI-01 & Final depth BGS: $18.04 \mathrm{ft}$ \\
\hline
\end{tabular}

\section{WCLIF13}

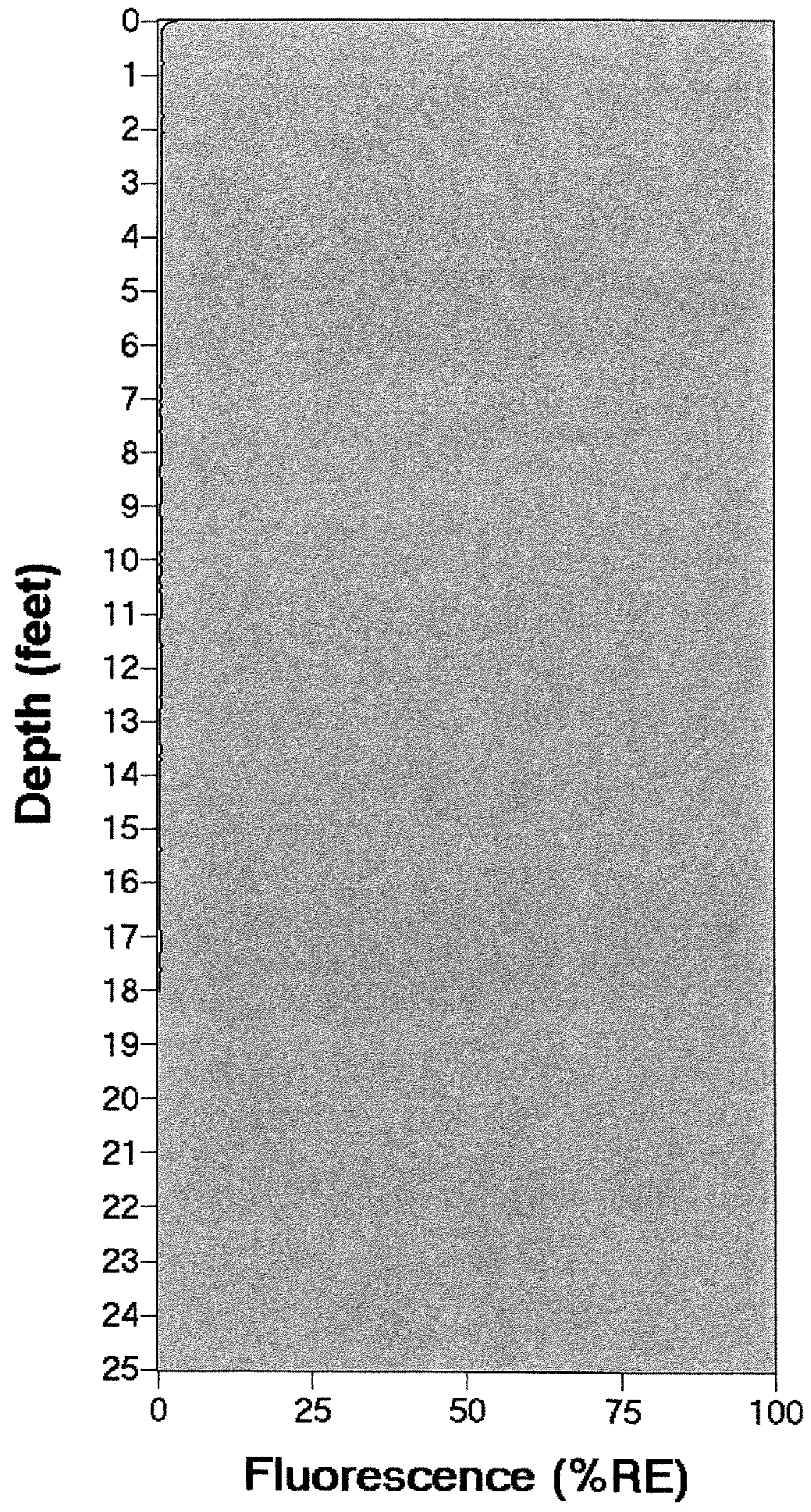

Dakota Technologies. Inc. 2201-A 12th St N.. Fargo. ND 58102 (701) 237-4908 wm. dakotatechnologies.com 
ROST Fluorescence Response Data

Site: CSI Watford City. ND

Client: EERC

Date/Time:11/10/2005@9:51:15 AM

ROST Unit: DTI-01
Operator: Steve Adamek

GPS FIX:

Max fluorescence: $16.25 \% @ 5.55 \mathrm{ft}$

Final depth BGS: $18.00 \mathrm{ft}$
WCLIF14

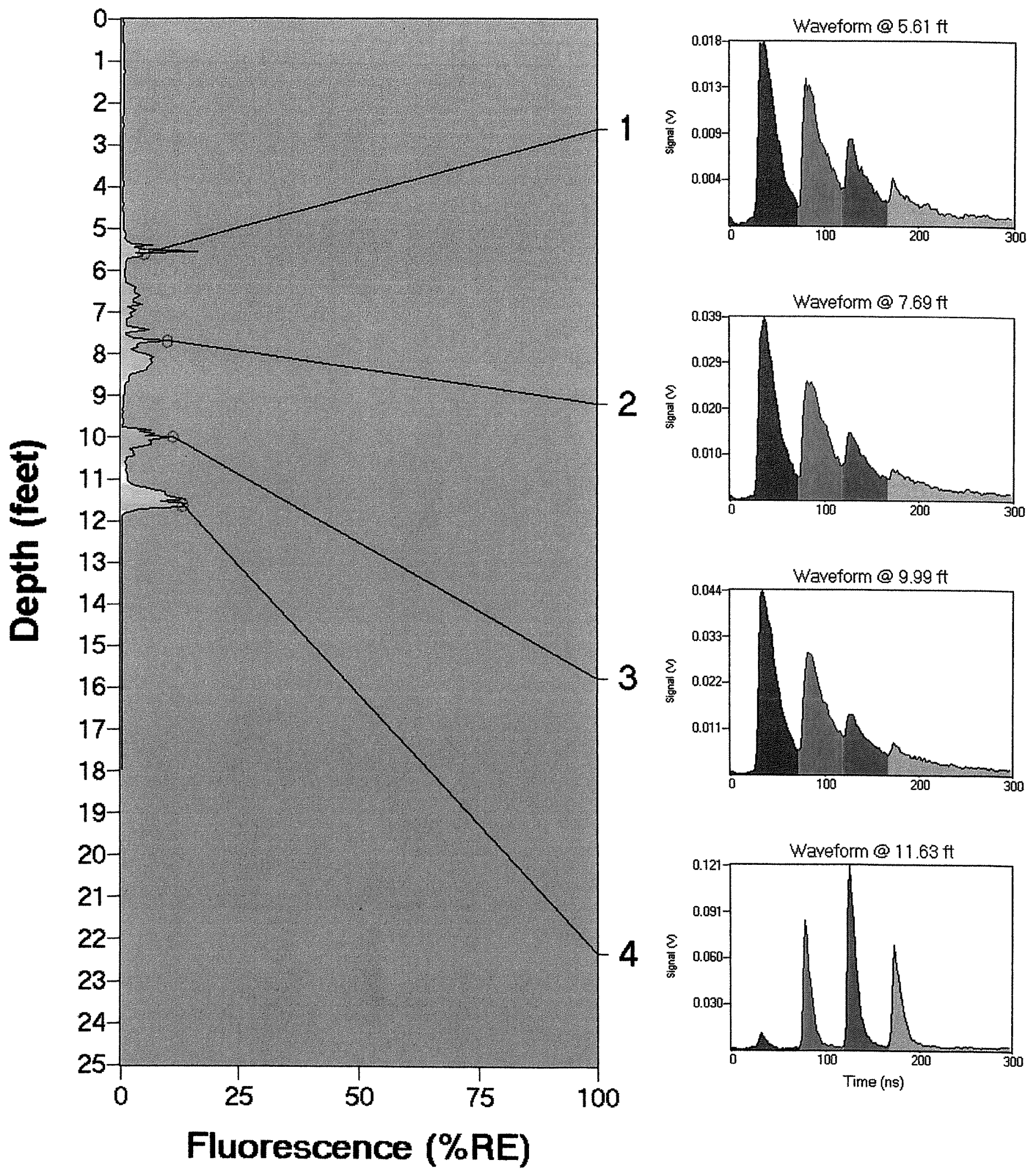


ROST Fluorescence Response Data

Site: CSI Watford City. ND

Client: EERC

DaterTime: 11/10/2005@10:14:59 AM

ROST Unit: DTI-01
Operator: Steve Adamek

GPS FIX:

Max fluorescence: $0.94 \% @ 0.74 \mathrm{ft}$

Final depth BGS: $17.95 \mathrm{ft}$

\section{WCLIF15}

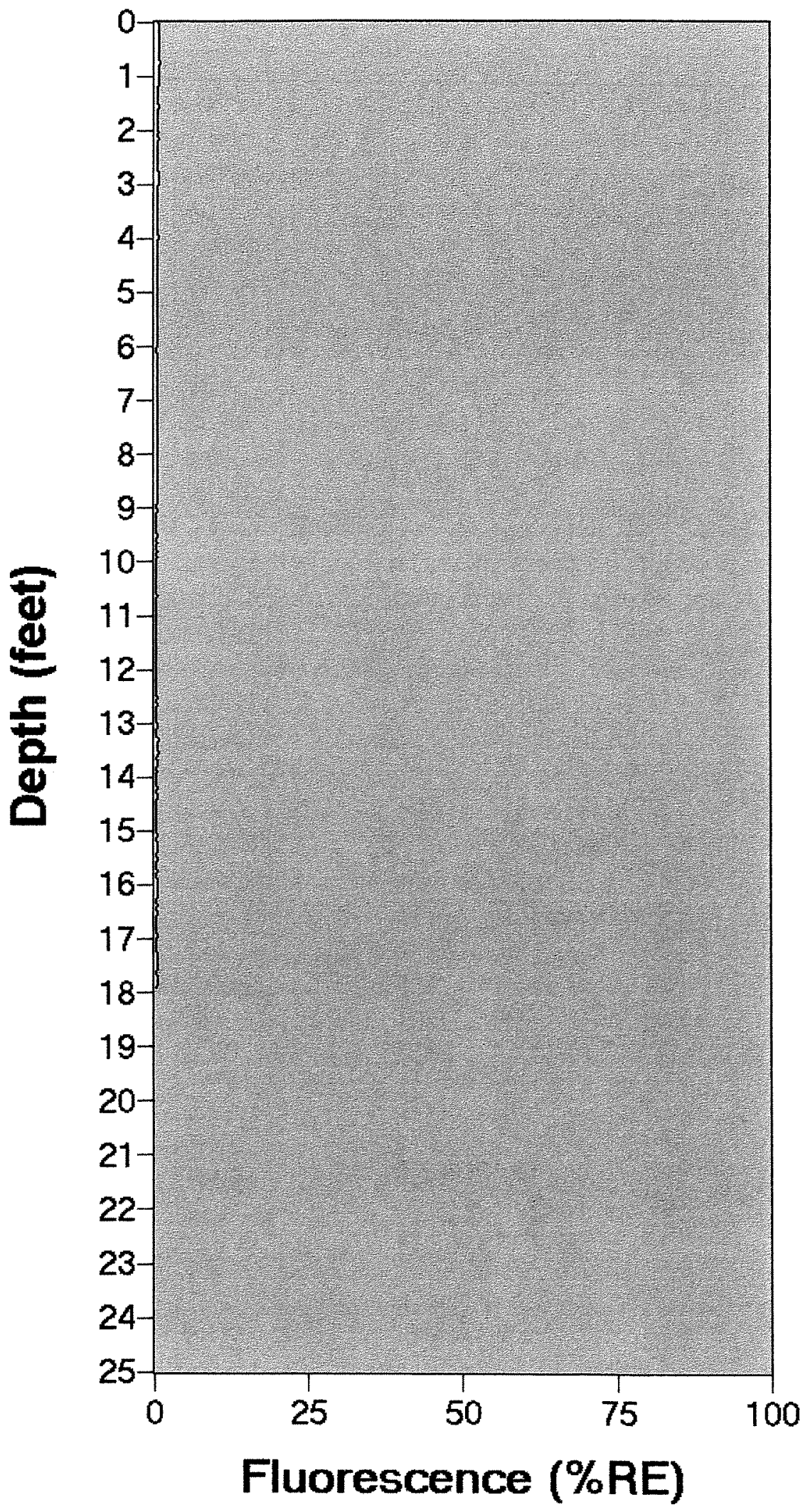

Dakota Technologies. Inc., 2201-A 12th St N. Fargo. ND 58102 (701) 237-4908 www dakotatechnologies.com 
ROST Fluorescence Response Data

\begin{tabular}{|l|l|}
\hline Site: CSI Watford City, ND & Operator: Steve Adamek \\
Client: EERC & GPS Fix: \\
Date/Time: $11 / 10 / 2005 @ 1033.09$ AM & Max fluorescence: $41.74 \% @ 11.28 \mathrm{ft}$ \\
ROST Unit: DTI-01 & Final depth BGS: $17.96 \mathrm{ft}$ \\
\hline
\end{tabular}

\section{WCLIF16}

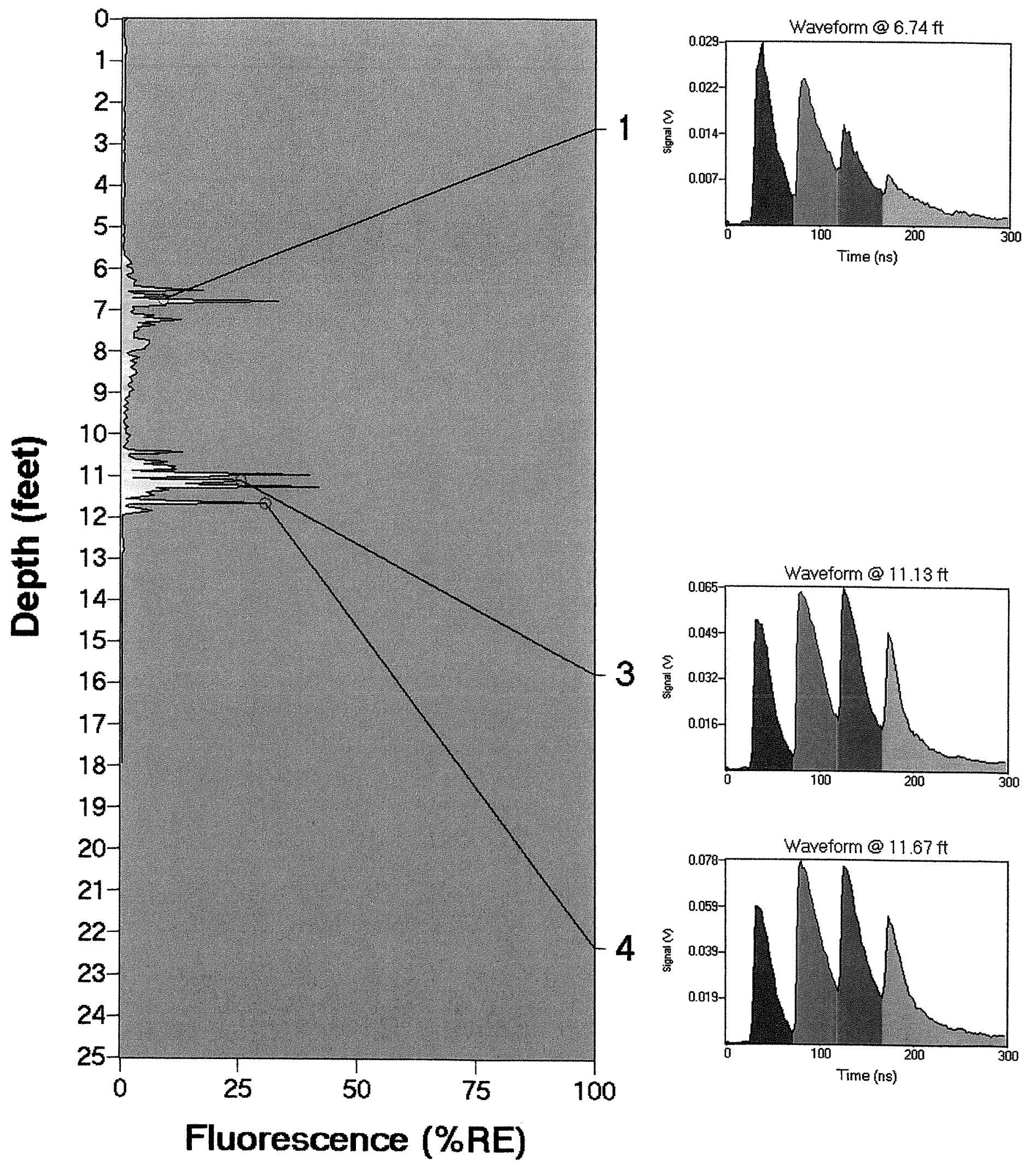

Dakota Technologies. Inc. 2201-A 12th St N.. Fargo. ND 58102 (701) 237-4908 ww dakotatechnologies.com 
ROST Fluorescence Response Data

\begin{tabular}{|l|l|}
\hline Site: CSI Watford City. ND & Operator: Steve Adamek \\
Client: EERC & GPS Fix: \\
Date/Time: $11 / 10 / 2005 @ 10: 54: 26$ AM & Max fluarescence: $1.81 \% @ 5.81 \mathrm{ft}$ \\
ROST Unit: DTI-01 & Final depth BGS: $17.81 \mathrm{ft}$ \\
\hline
\end{tabular}

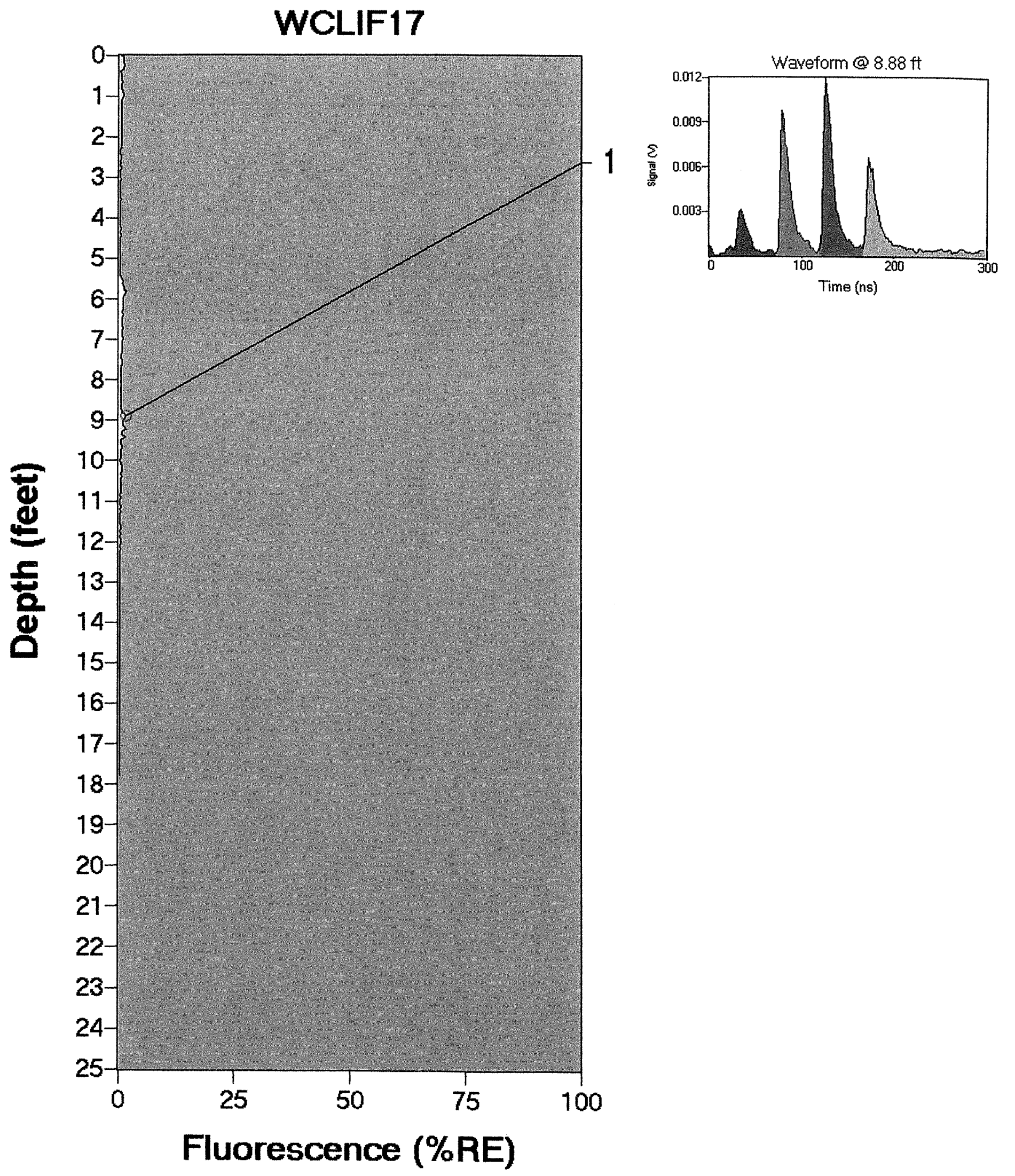


ROST Fluorescence Response Data

Site: CSI Watford City. ND

Client: EERC

Date/Time:11/10/2005@11:12:46 AM

ROST Unit: DTI-01
Operator: Steve Adamek

GPS FIX:

Max fluorescence: $2.98 \% @ 7.12 \mathrm{ft}$

Final depth BGS: $18.00 \mathrm{ft}$

\section{WCLIF18}

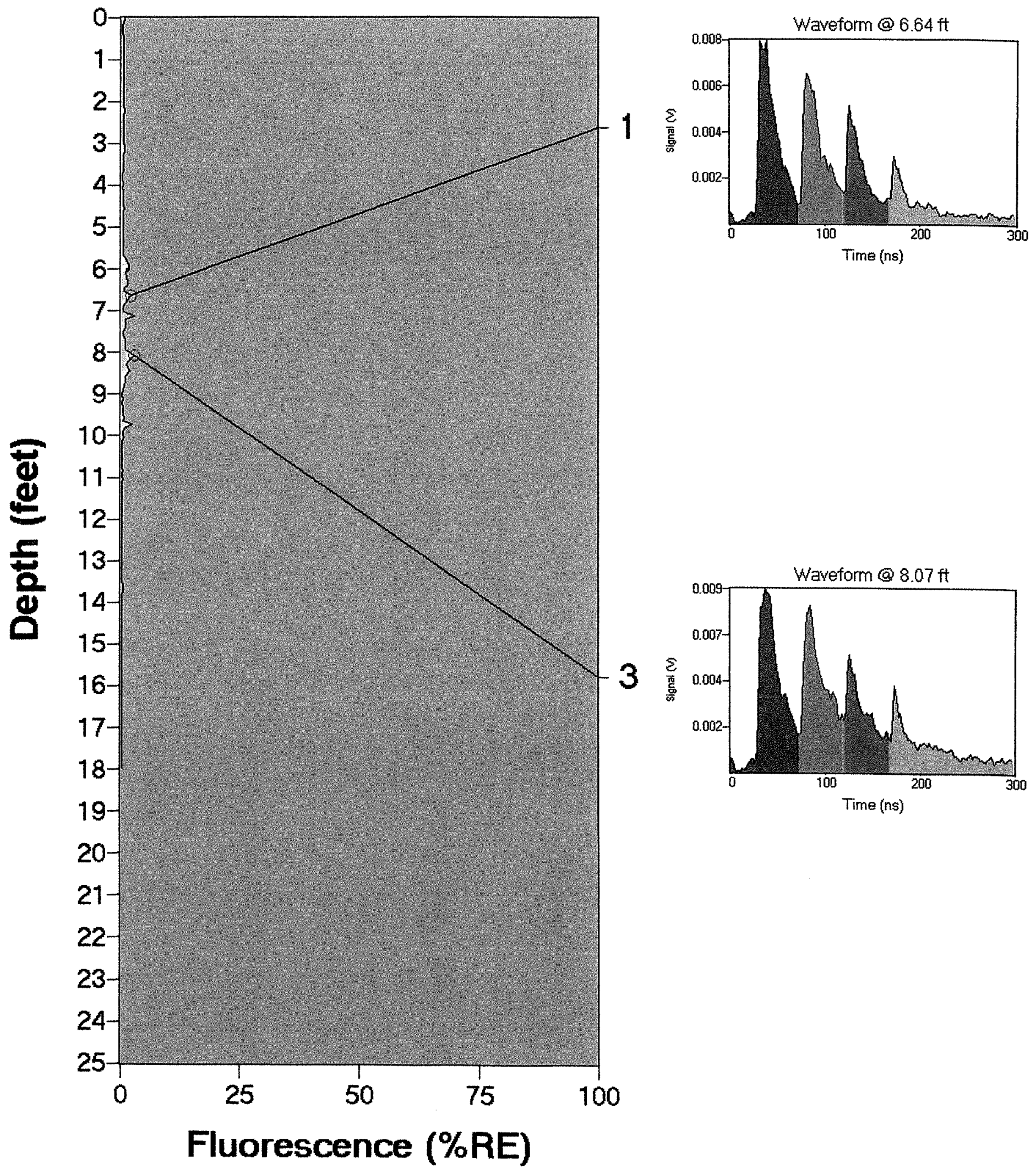


Site: CSI Watford City. ND

Client: EERC

Date/Time: 11/10/2005@ 11:32:56 AM

ROST Unit: DTI-01
Operator: Steve Adamek

GPS FIX:

Max fluorescence: $8.05 \% @ 12.62 \mathrm{ft}$

Final depth BGS: $17.97 \mathrm{ft}$
WCLIF19

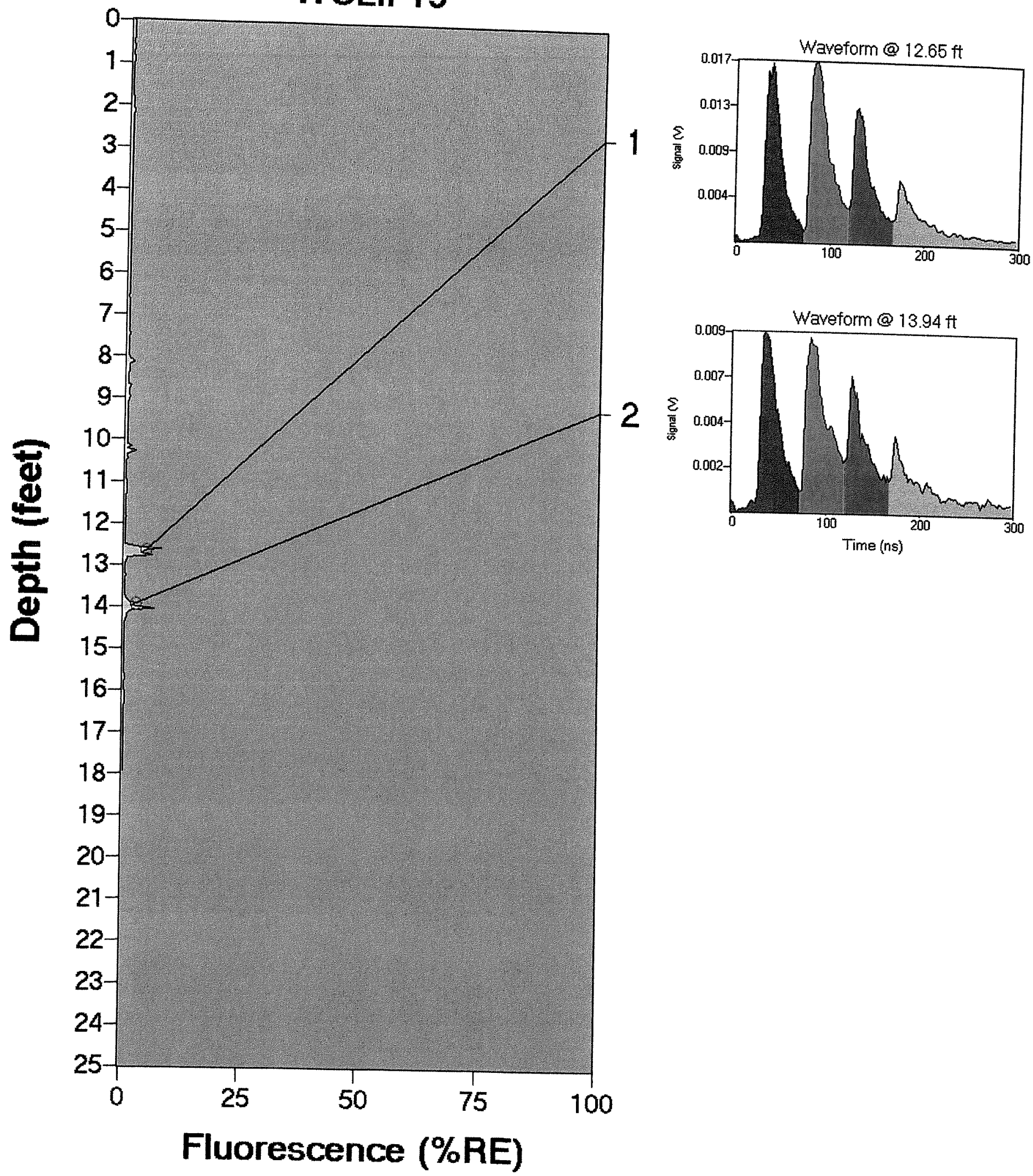




Site: CSI Watford City. ND
Client: EERC
Date/Time: 11/10/2005@11:51:54 AM
ROST Unit: DTI-01

Operator: Steve Adamek

GPS FIX:

Max fluorescence: $52.05 \% @ 7.68 \mathrm{ft}$

Final depth BGS: $17.94 \mathrm{ft}$

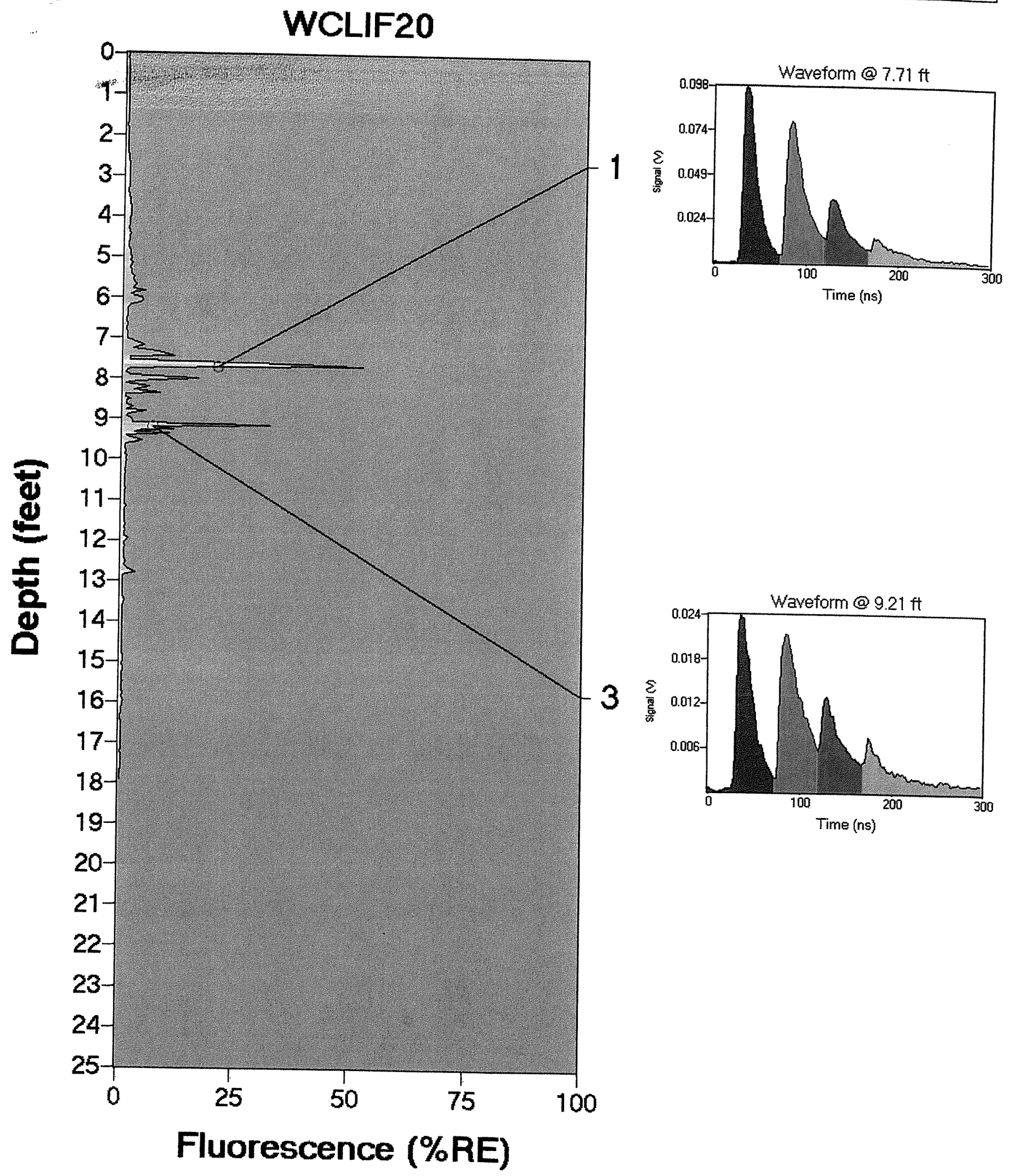

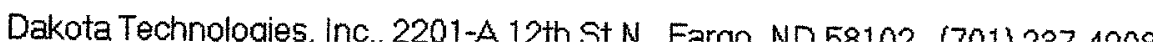


ROST Fluorescence Response Data

\begin{tabular}{|l|l|}
\hline Site: CSI Watford City. ND & Operator: Steve Adamek \\
Client: EERC & GPS Fix: \\
DaterTime: $11 / 10 / 2005 @ 12: 10: 41$ PM & Max fluorescence: $4.67 \% @ 10.22 \mathrm{ft}$ \\
ROST Unit: DTI-01 & Final depth BGS: $17.85 \mathrm{ft}$ \\
\hline
\end{tabular}

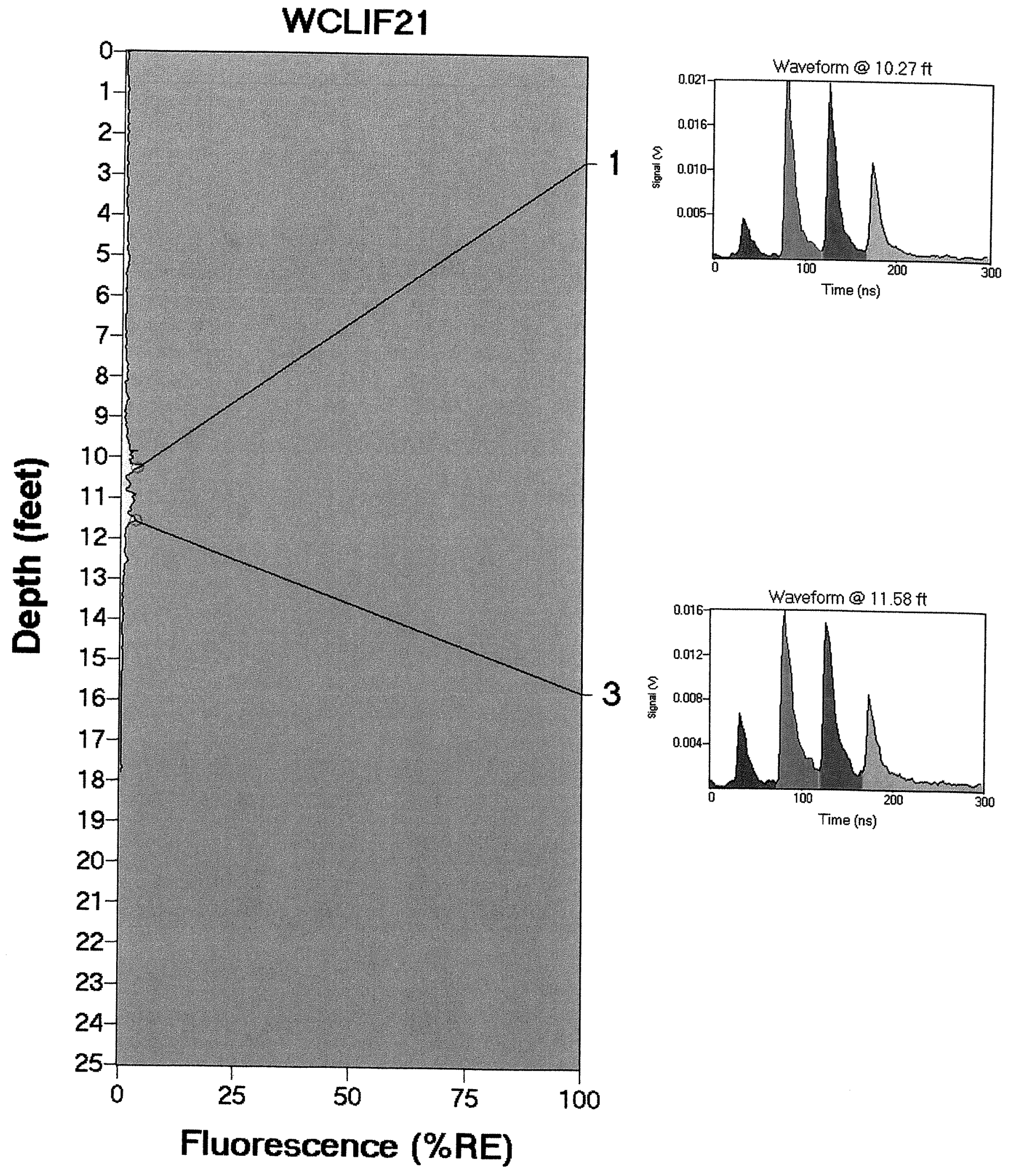

Dakota Technologies. Inc. 2201-A 12th St N.. Fargo. ND 58102 (701) 237-4908 whw dakntatechnninniec mm 
ROST Fluorescence Response Data

\begin{tabular}{l|l}
\hline Site: CSI Watford City. ND & Operator: Steve Adamek \\
Client: EERC & GPS Fix: \\
Date/Time: $11 / 10 / 2005 @ 12: 28: 50 \mathrm{PM}$ & Max fluorescence: $44.57 \% @ 10.50 \mathrm{ft}$ \\
ROST Unit: DTI-01 & Final depth BGS: $20.00 \mathrm{ft}$
\end{tabular}

\section{WCLIF22}

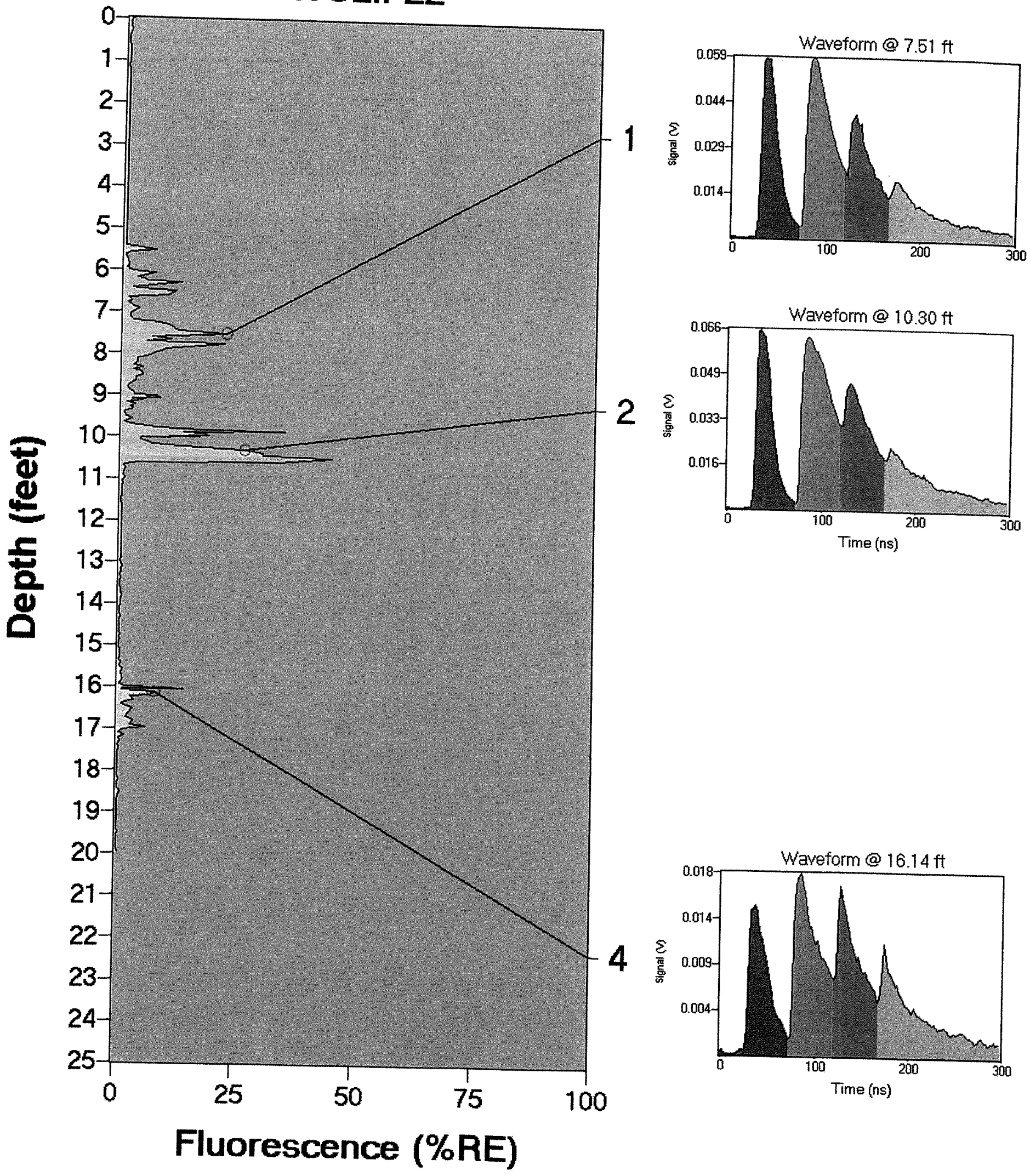

Dakota Technologies. Inc.. 2201-A 12th St N.. Fargo. ND 58102 (701) 237-4908 whw. dakotatechnologies.com 
ROST Fluorescence Response Data

Site: CSI Watford City. ND

Client: EERC

DaterTime:11/10/2005@12:51:24 PM

ROST Unit: DTI-01
Operator: Steve Adamek

GPS Fix:

Max fluorescence: $46.91 \% @ 8.60 \mathrm{ft}$

Final depth BGS: $20.01 \mathrm{ft}$

\section{WCLIF23}

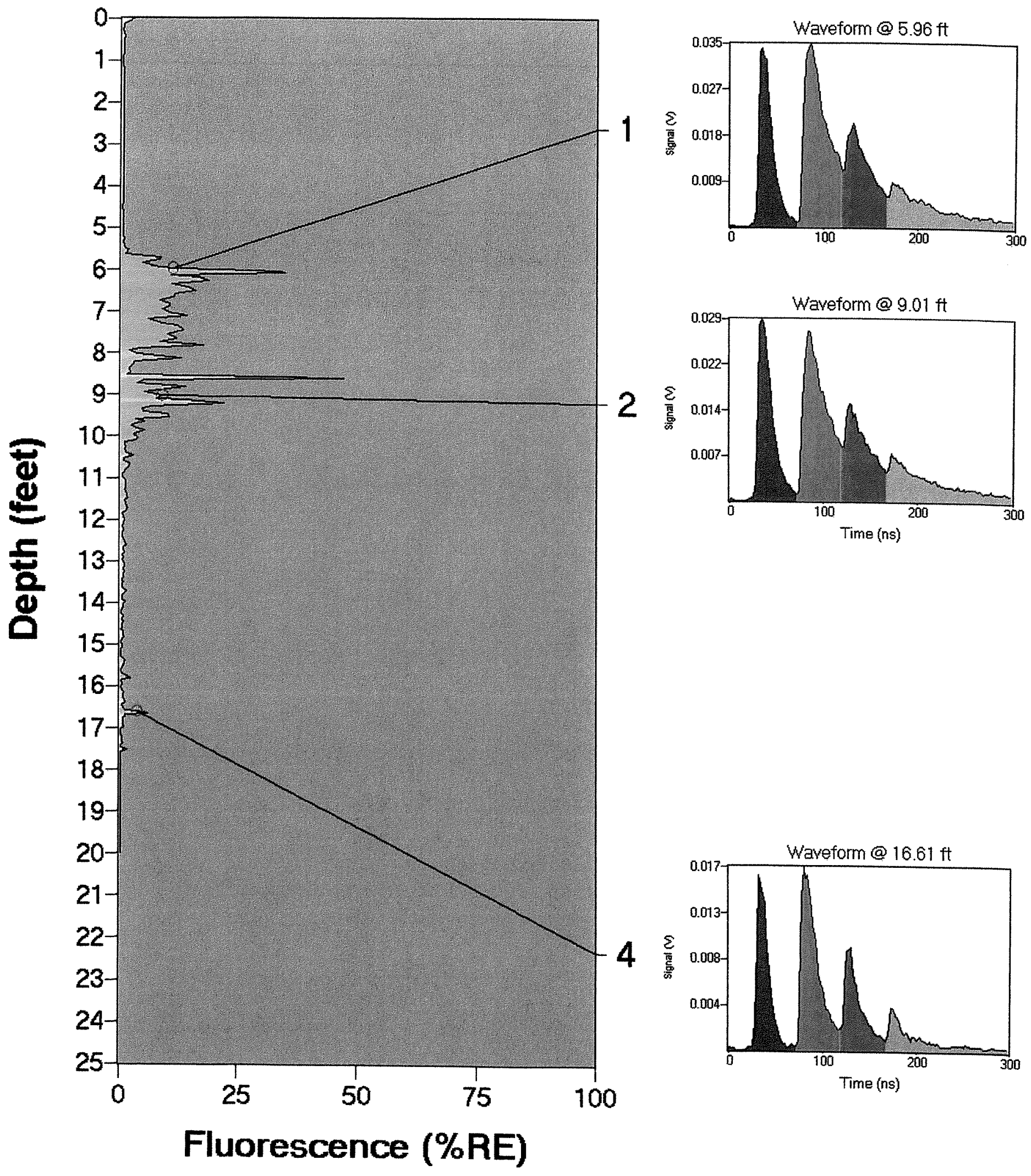


ROST Fluorescence Response Data

\begin{tabular}{l|l}
\hline Site: CSI Watford City. ND & Operator: Steve Adamek \\
Client: EERC & GPS FIx: \\
Date/Time: $11 / 10 / 2005 @ 2: 05: 46$ PM & Max fluorescence: $3.61 \% @ 5.78 \mathrm{ft}$ \\
ROST Unit: DTI-01 & Final depth BGS: $17.88 \mathrm{ft}$ \\
\hline
\end{tabular}

\section{WCLIF24}

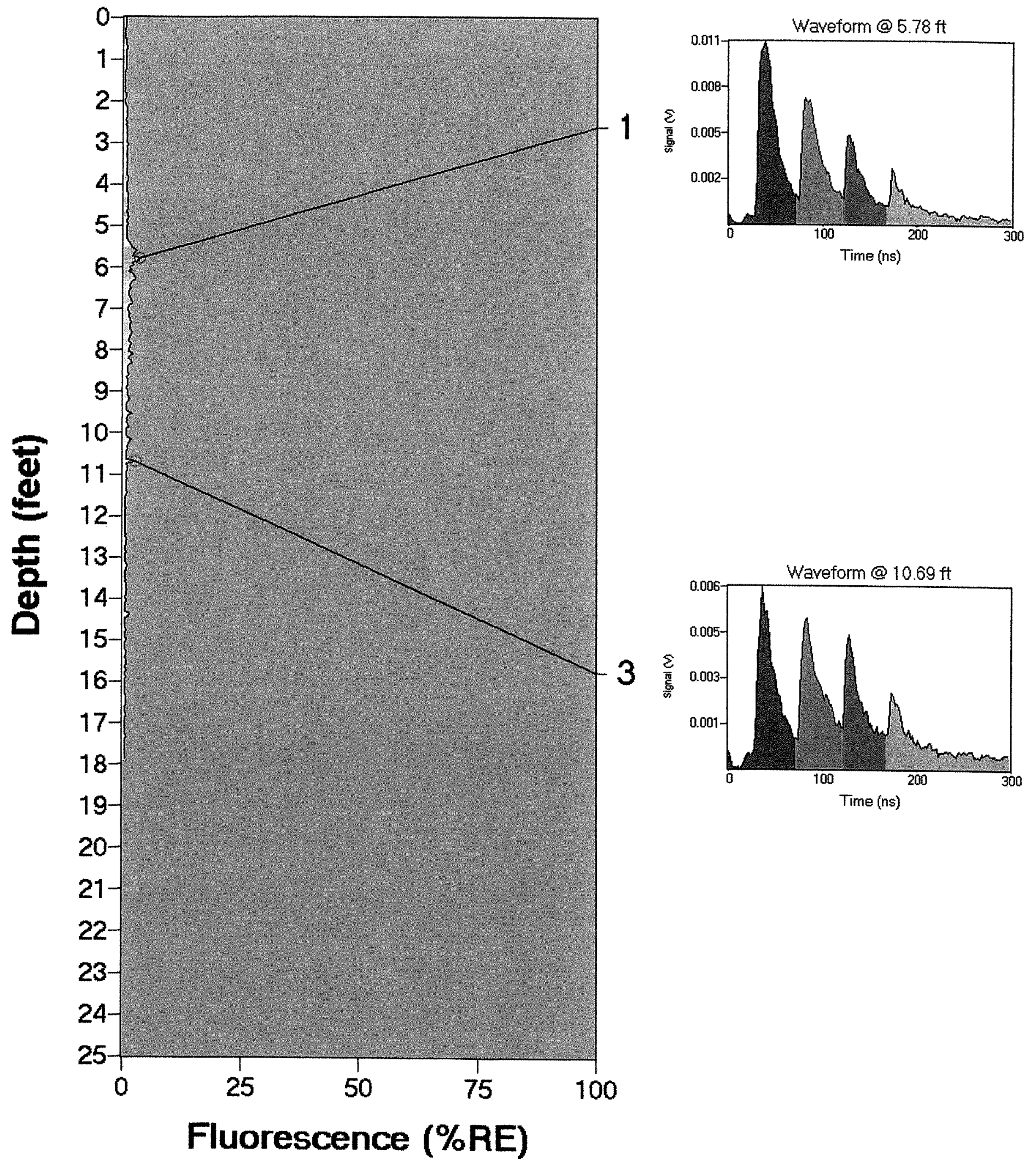

Dakota Technologies. Inc. 2201-A 12th St N.. Fargo. ND 58102 (701) 237-4908 www dakotatechnologies.com 


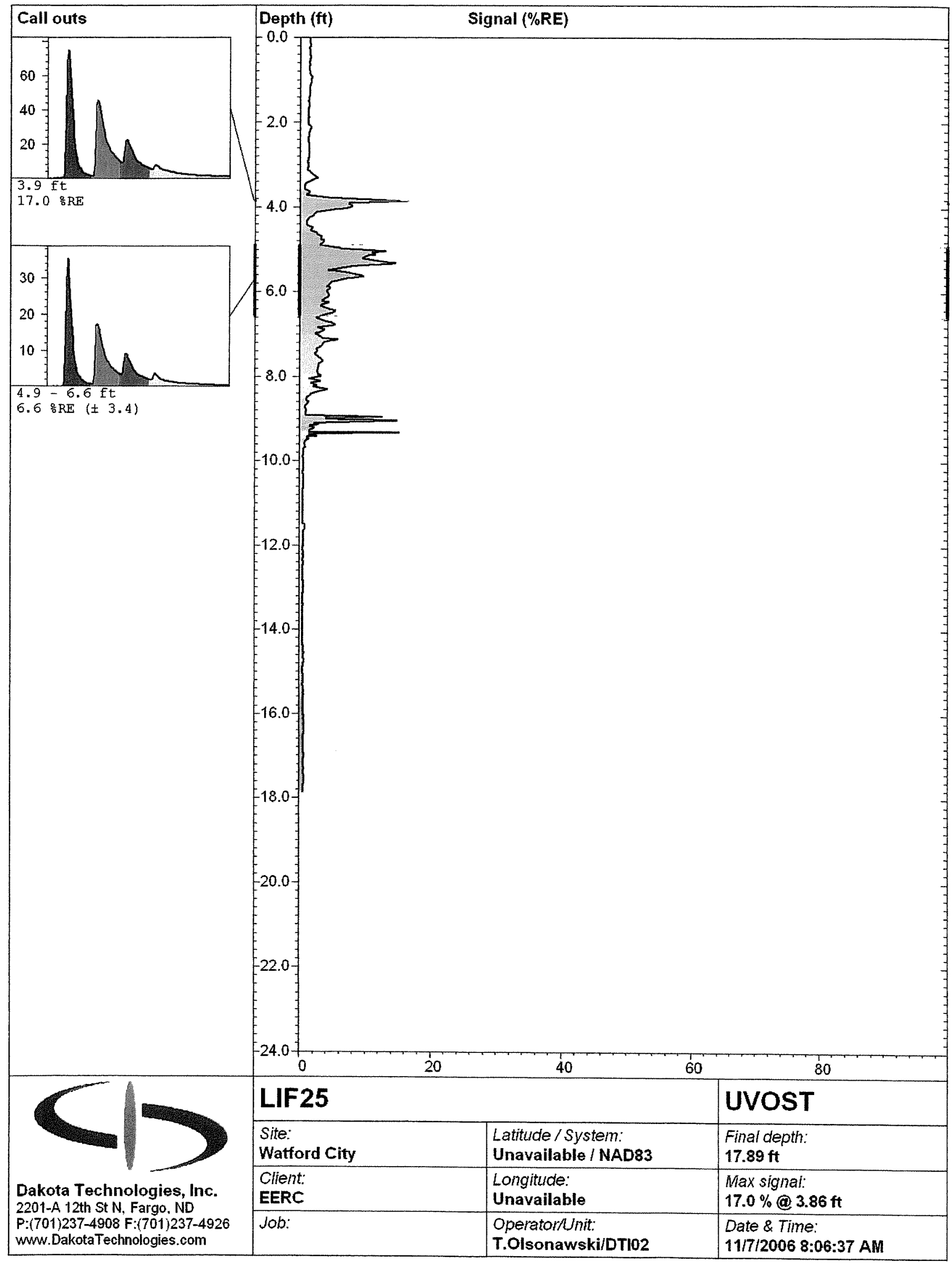




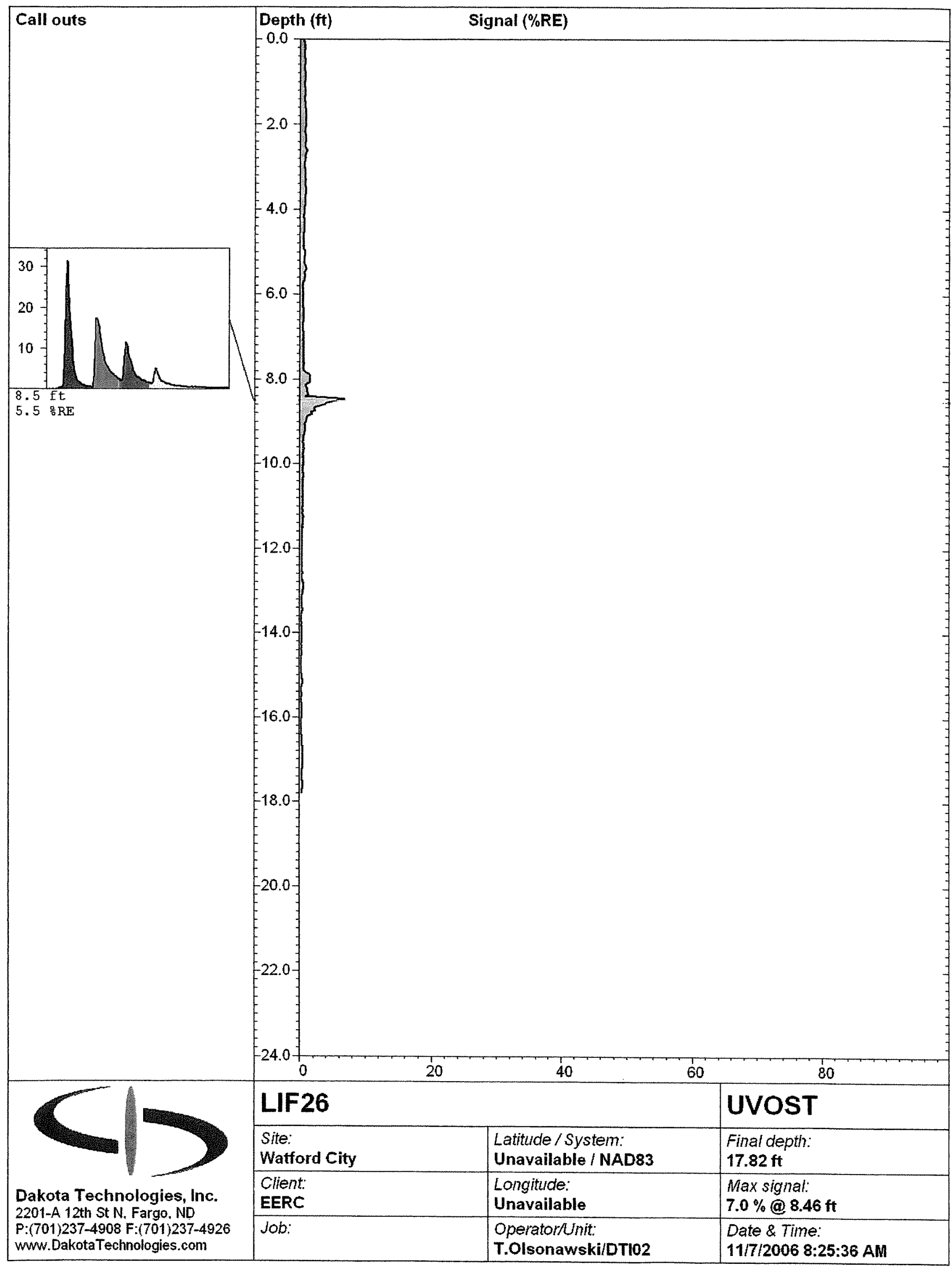




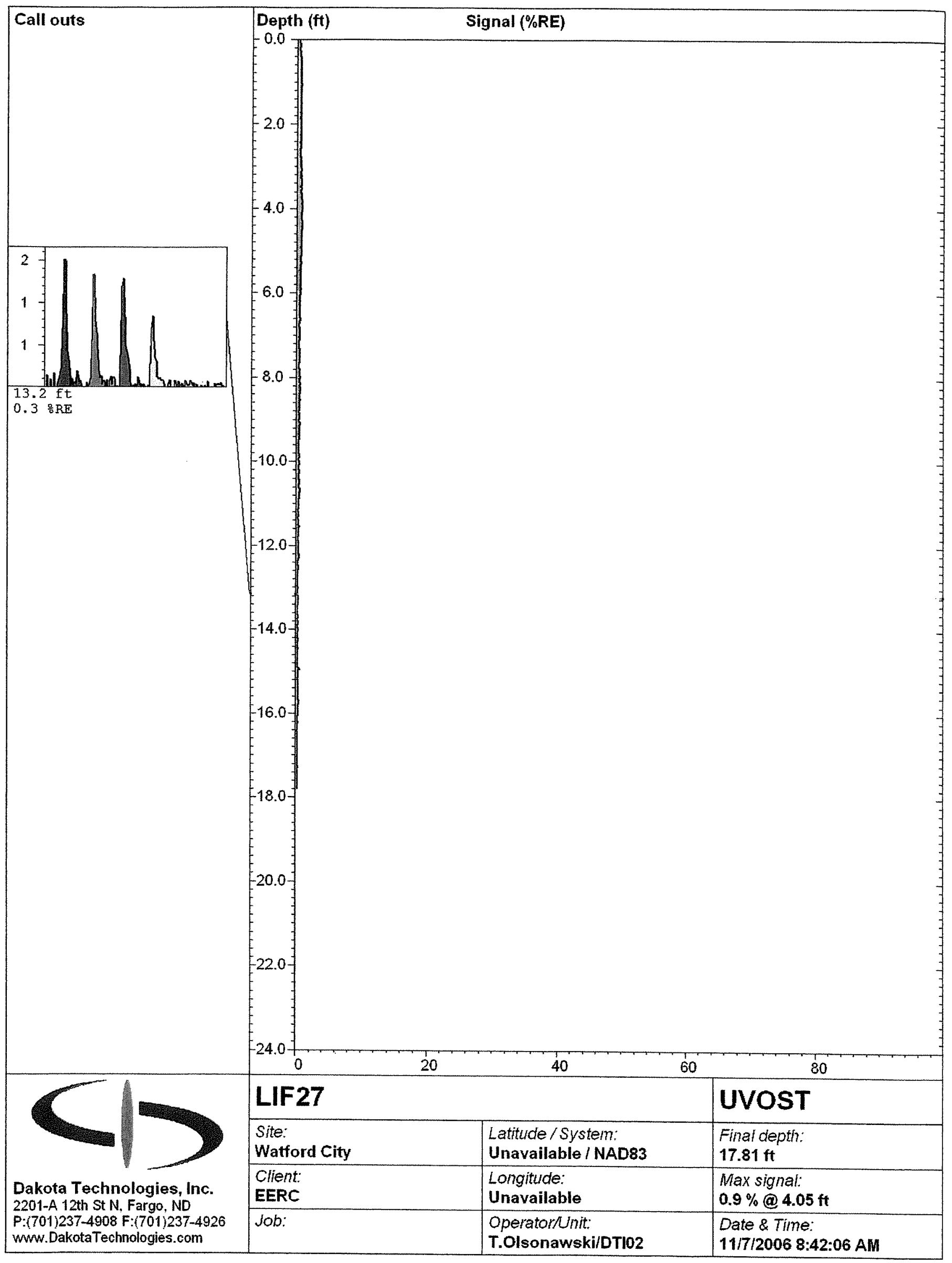




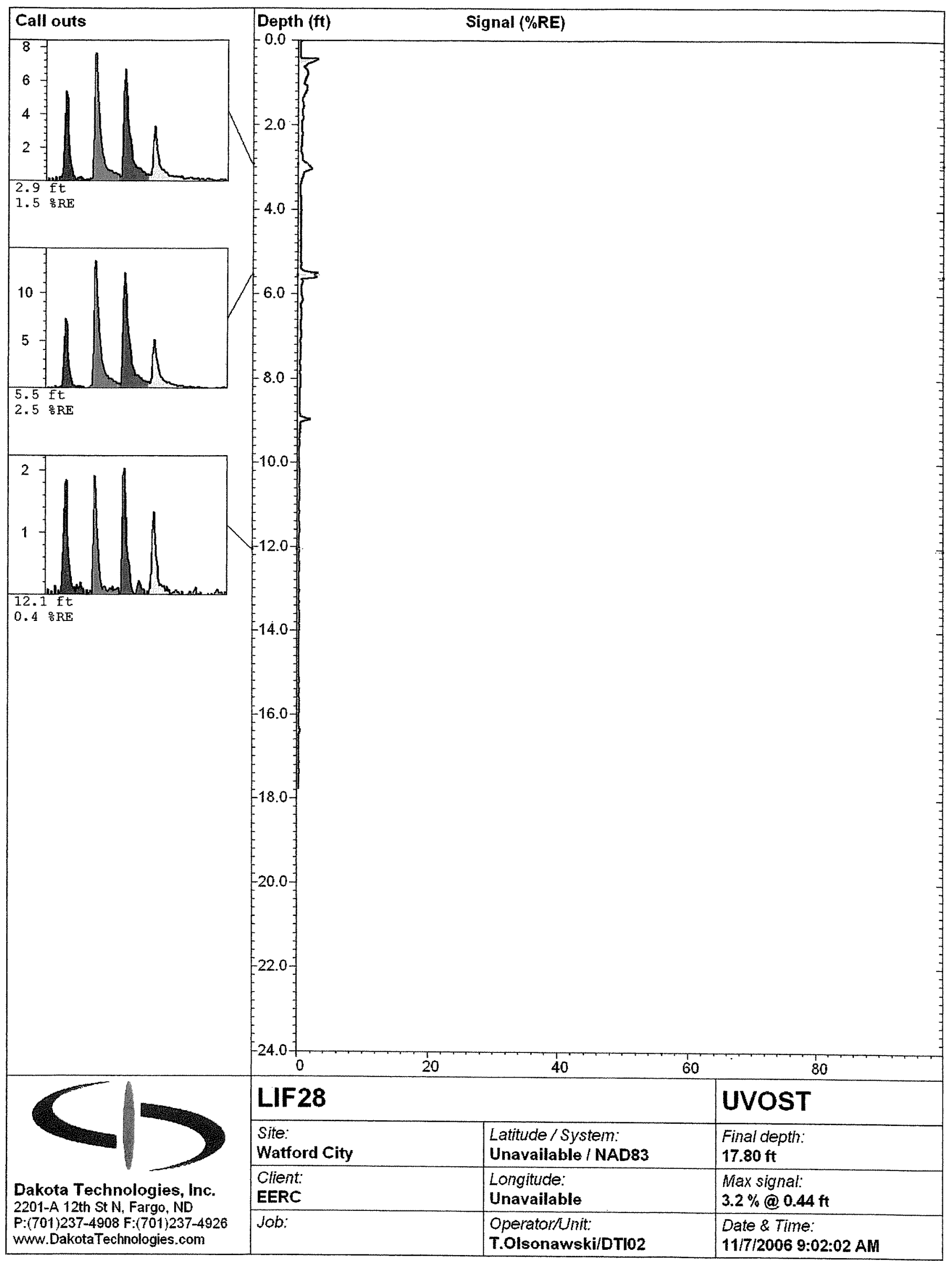




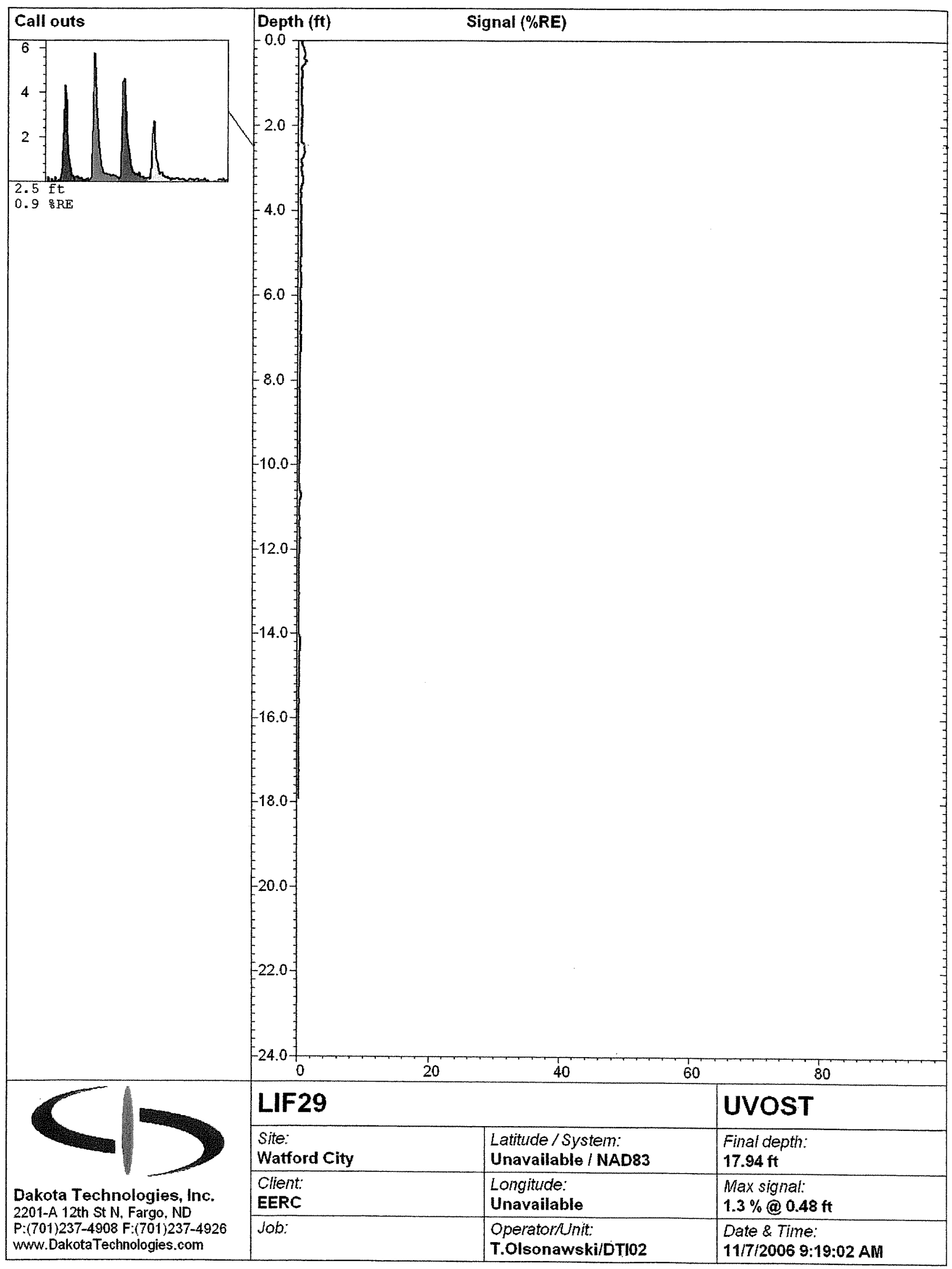




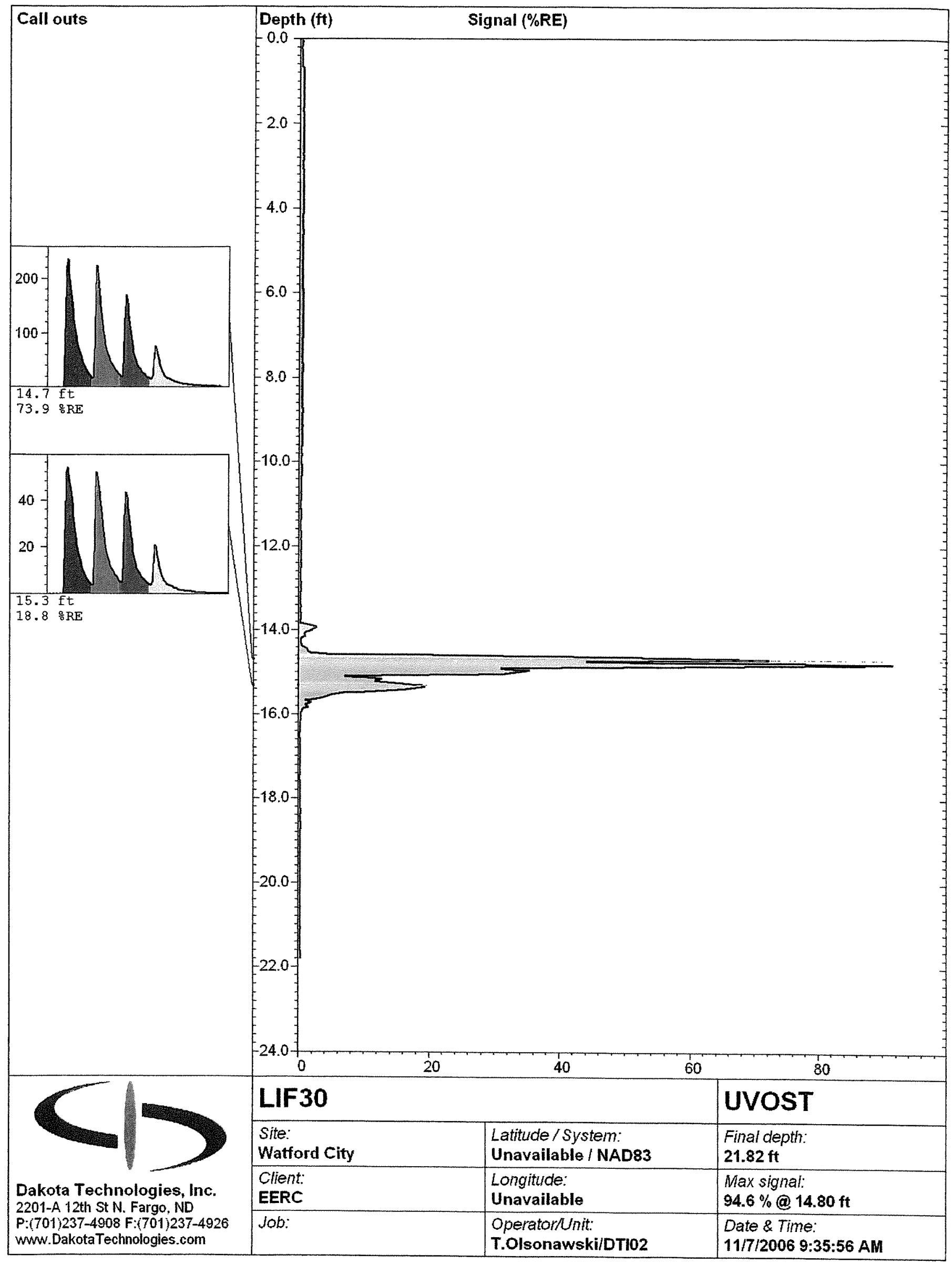




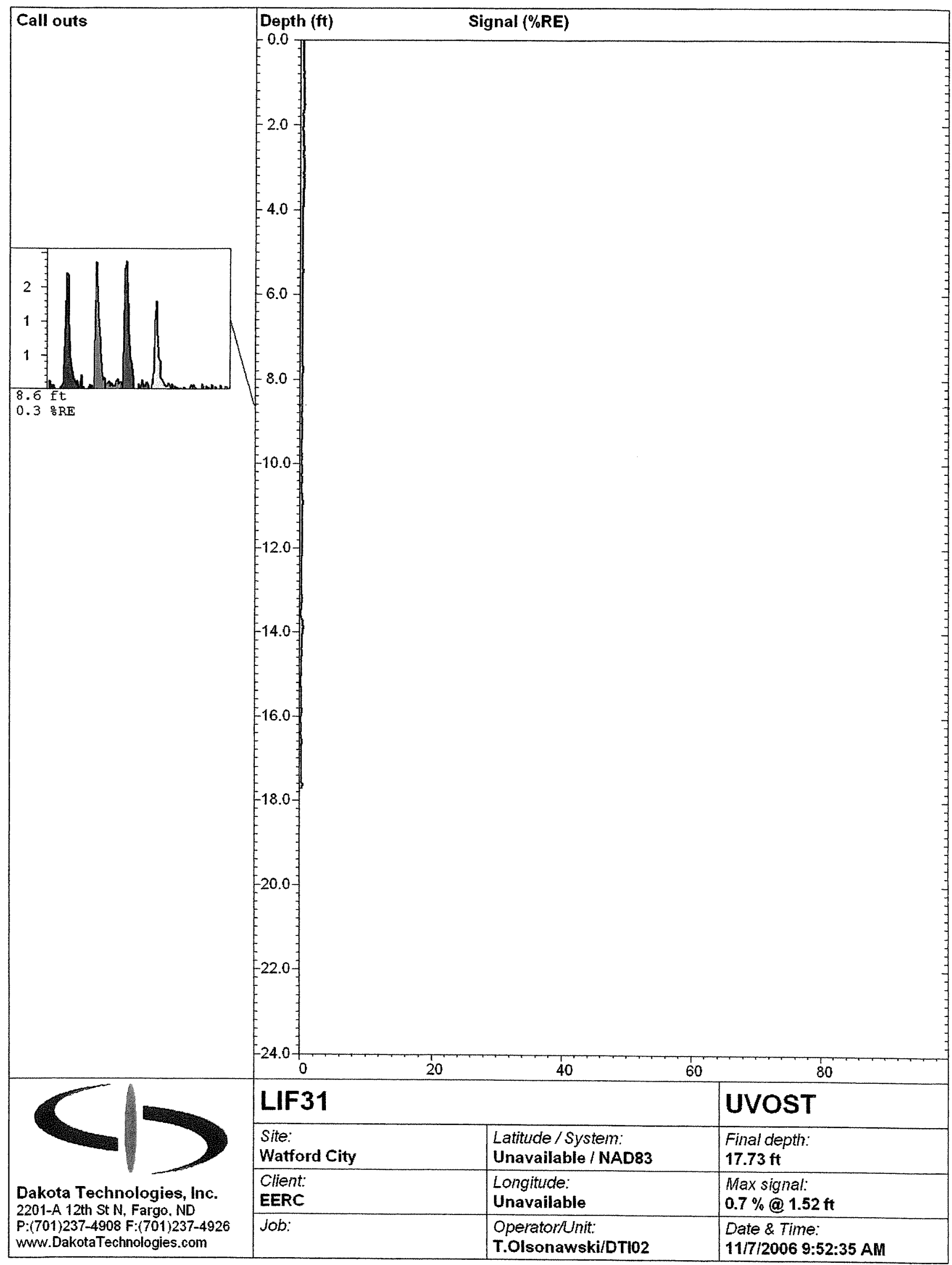




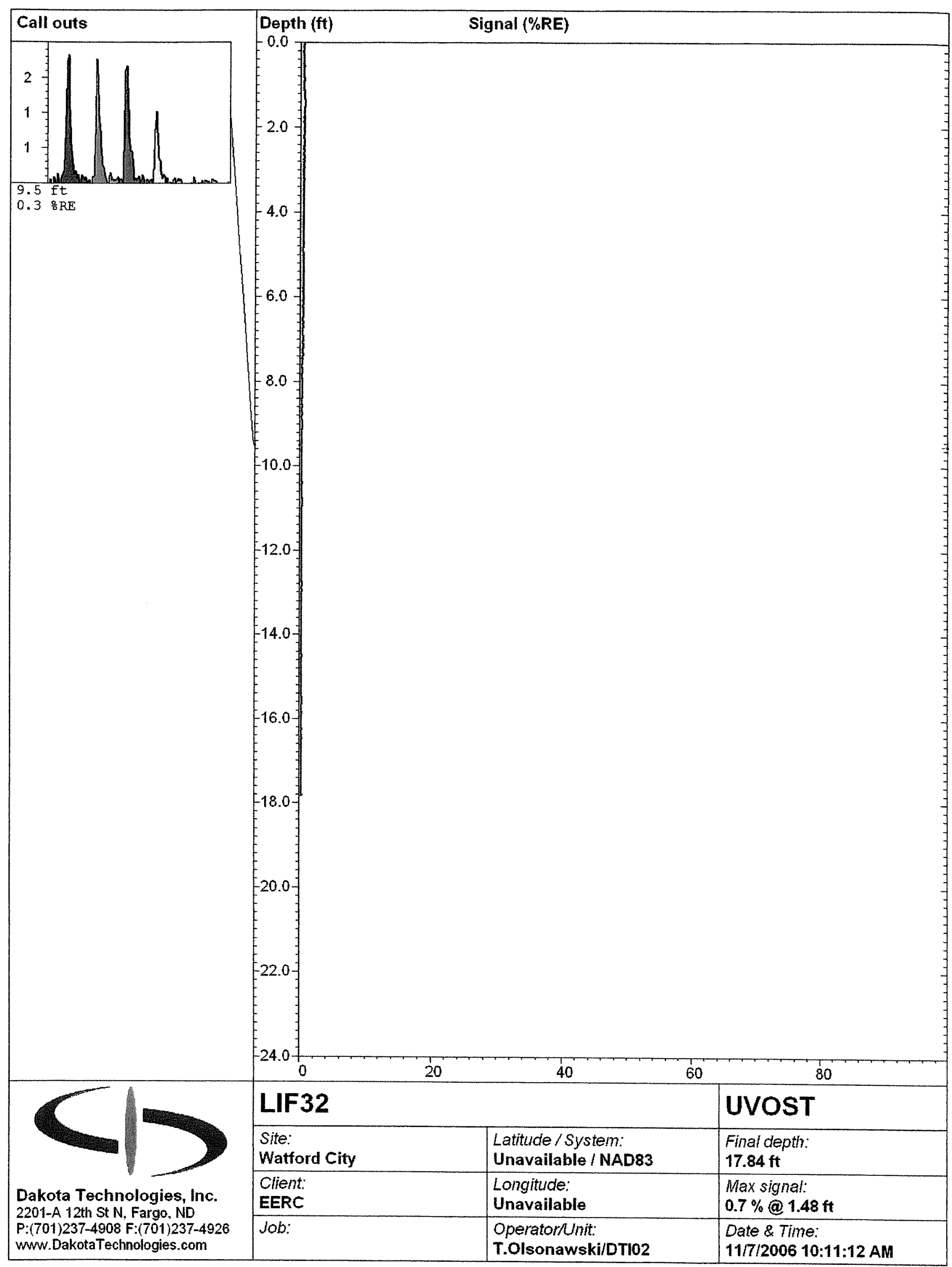




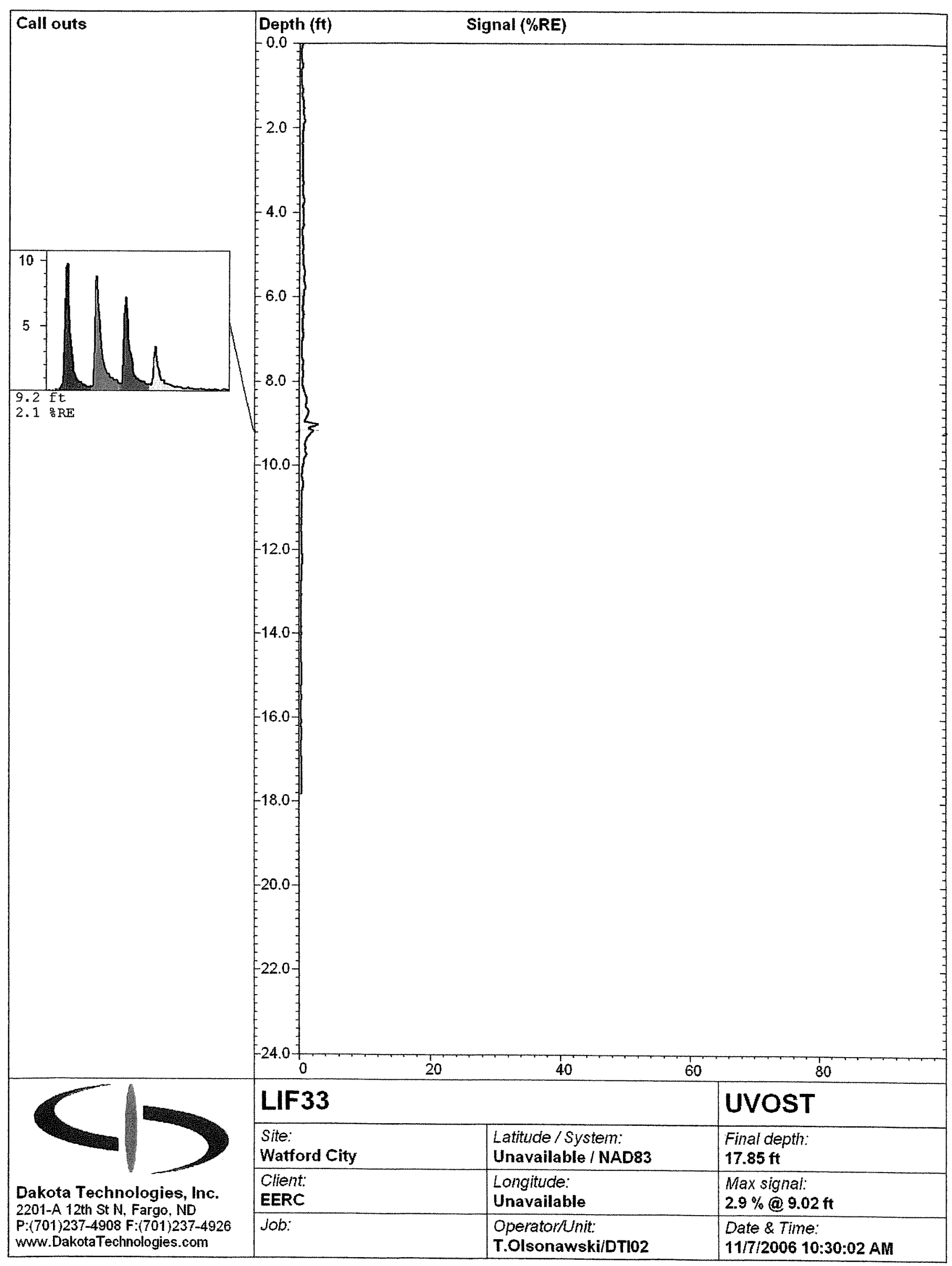




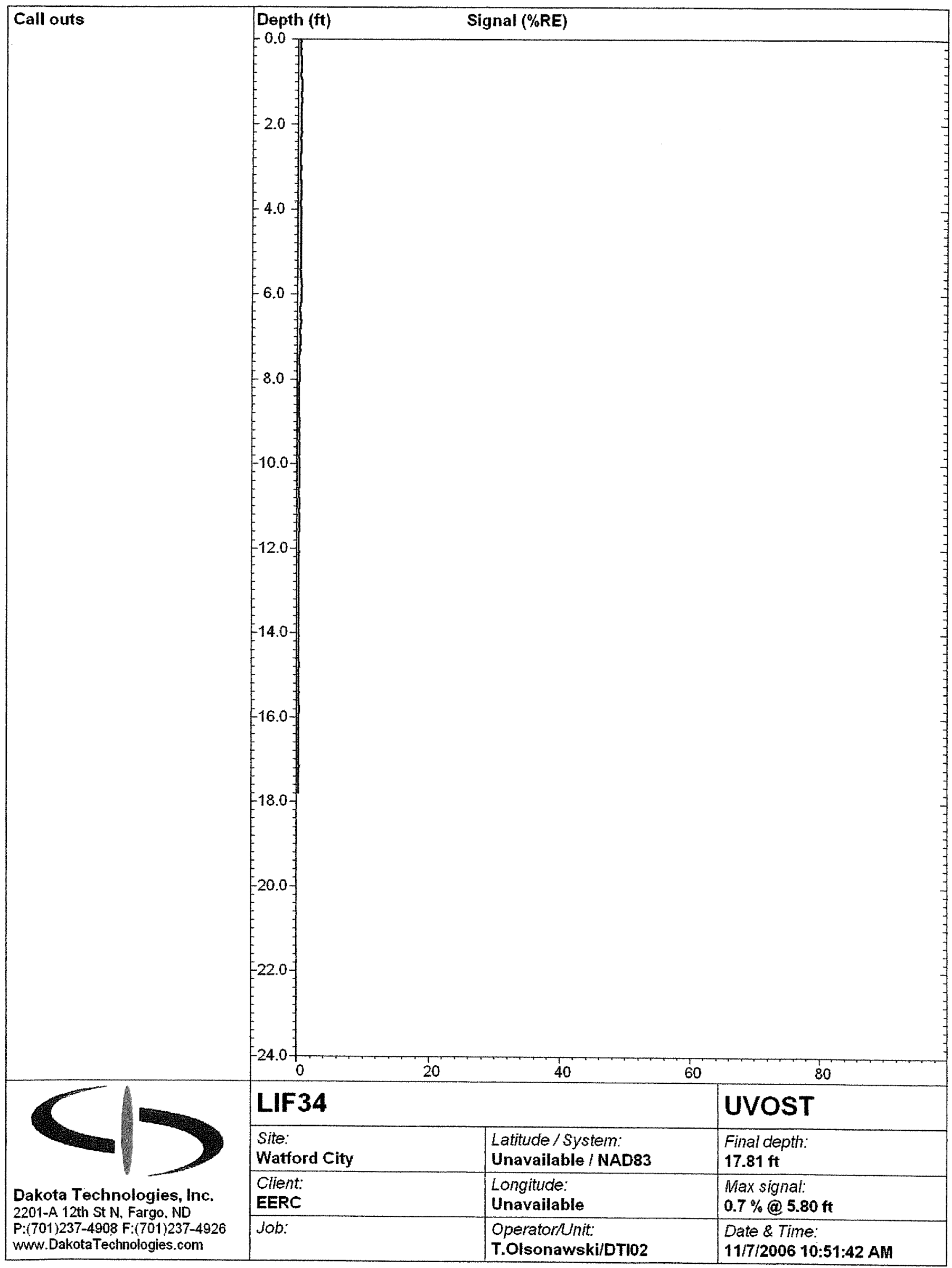




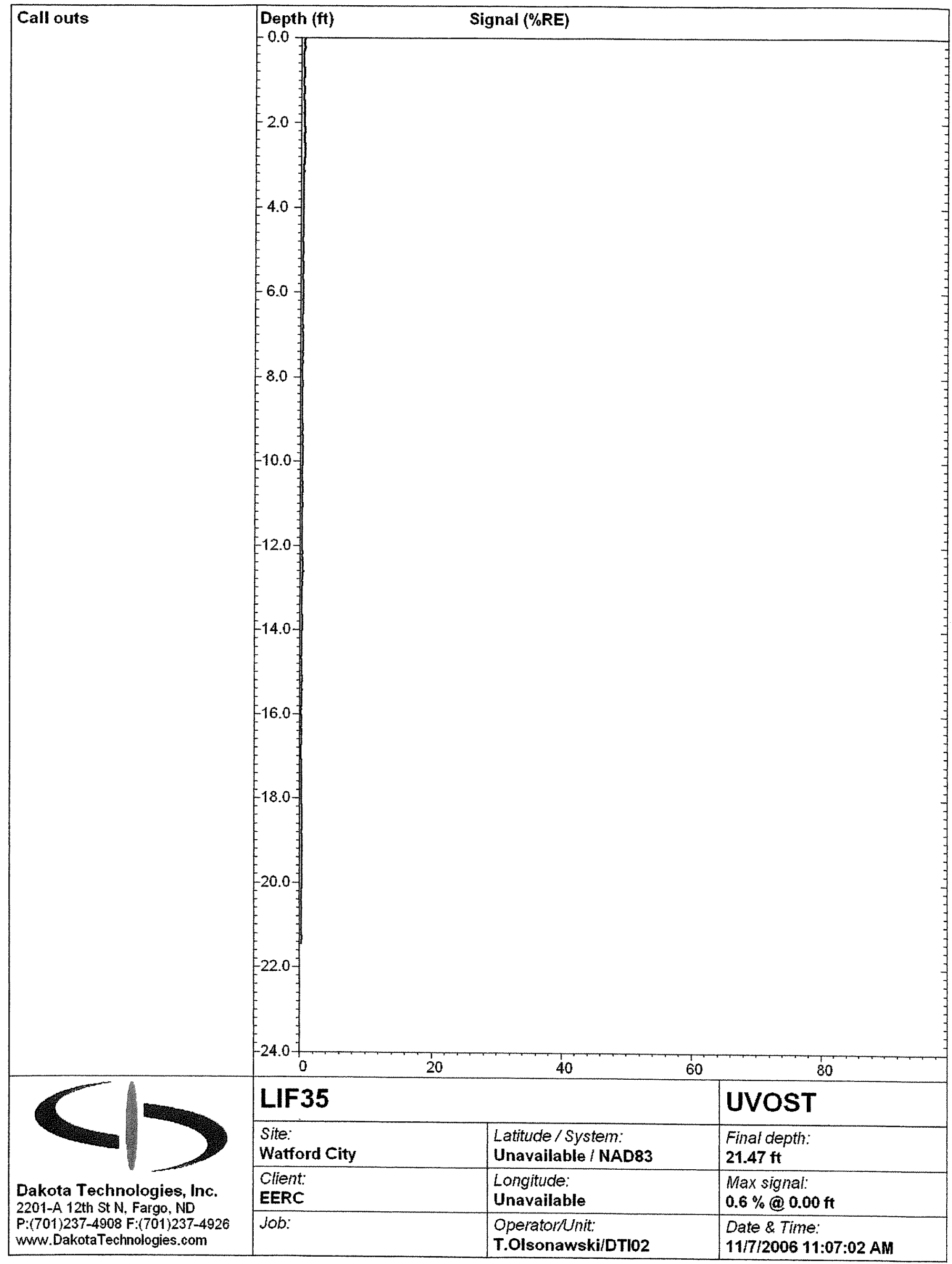




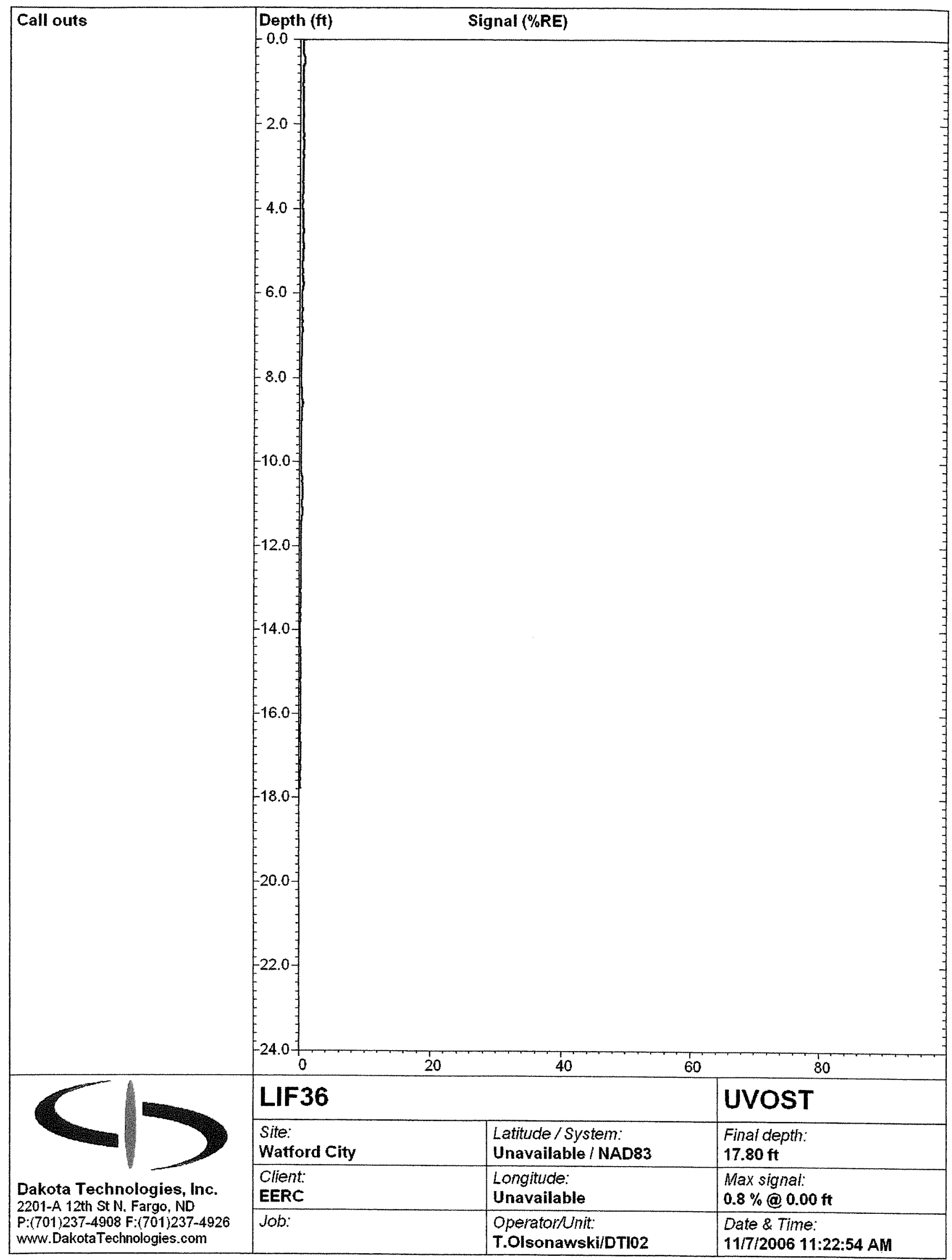




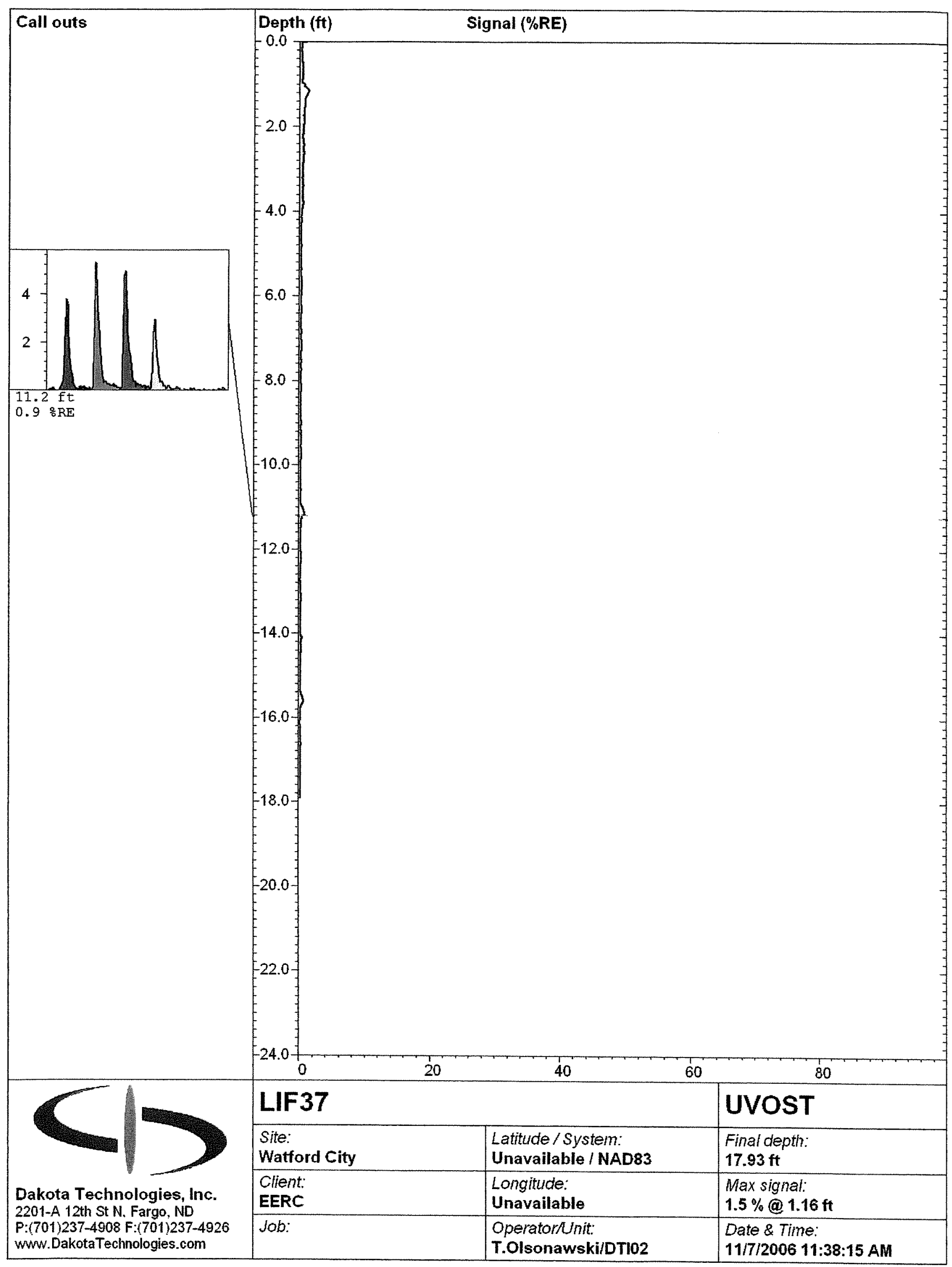




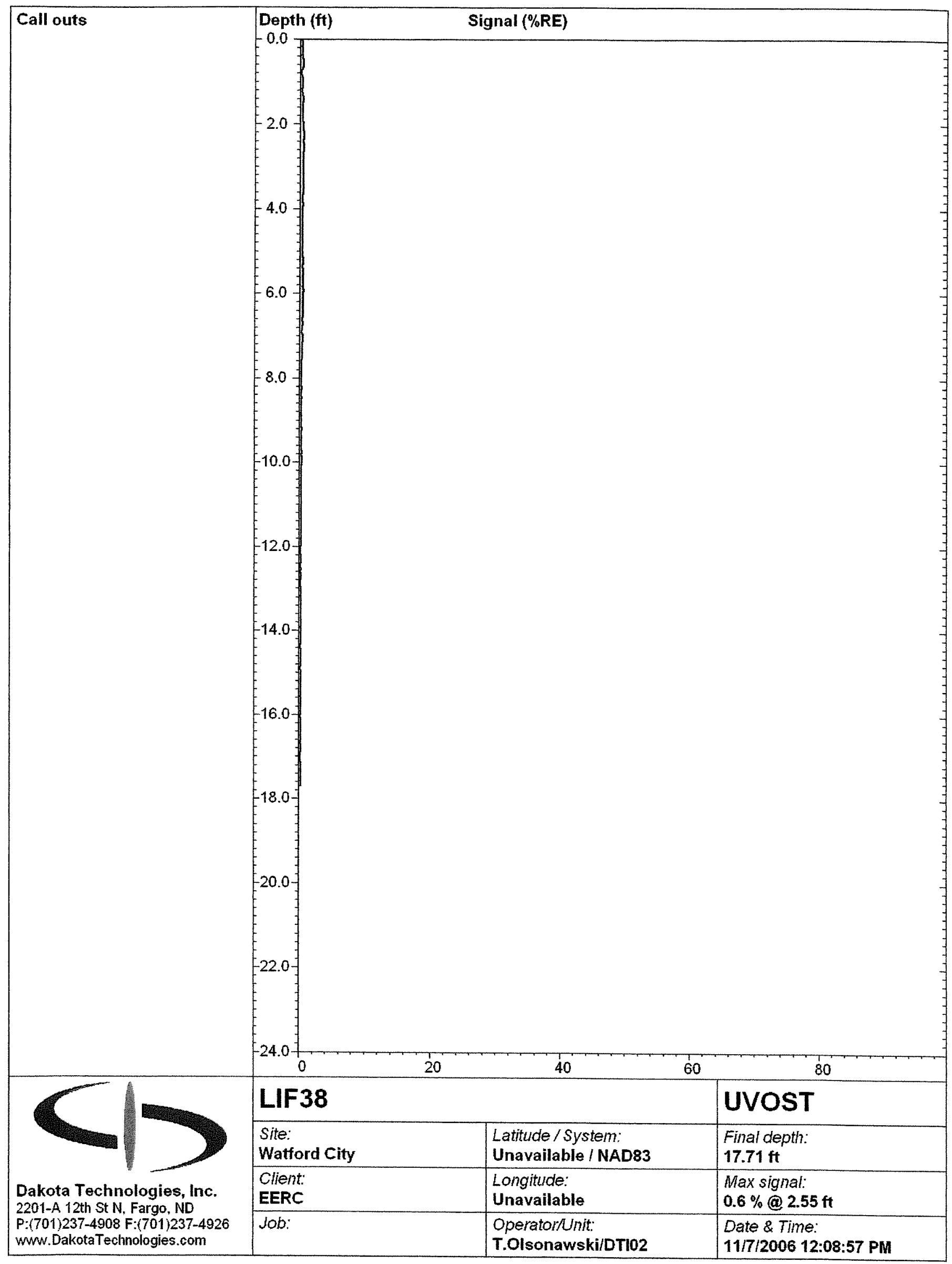




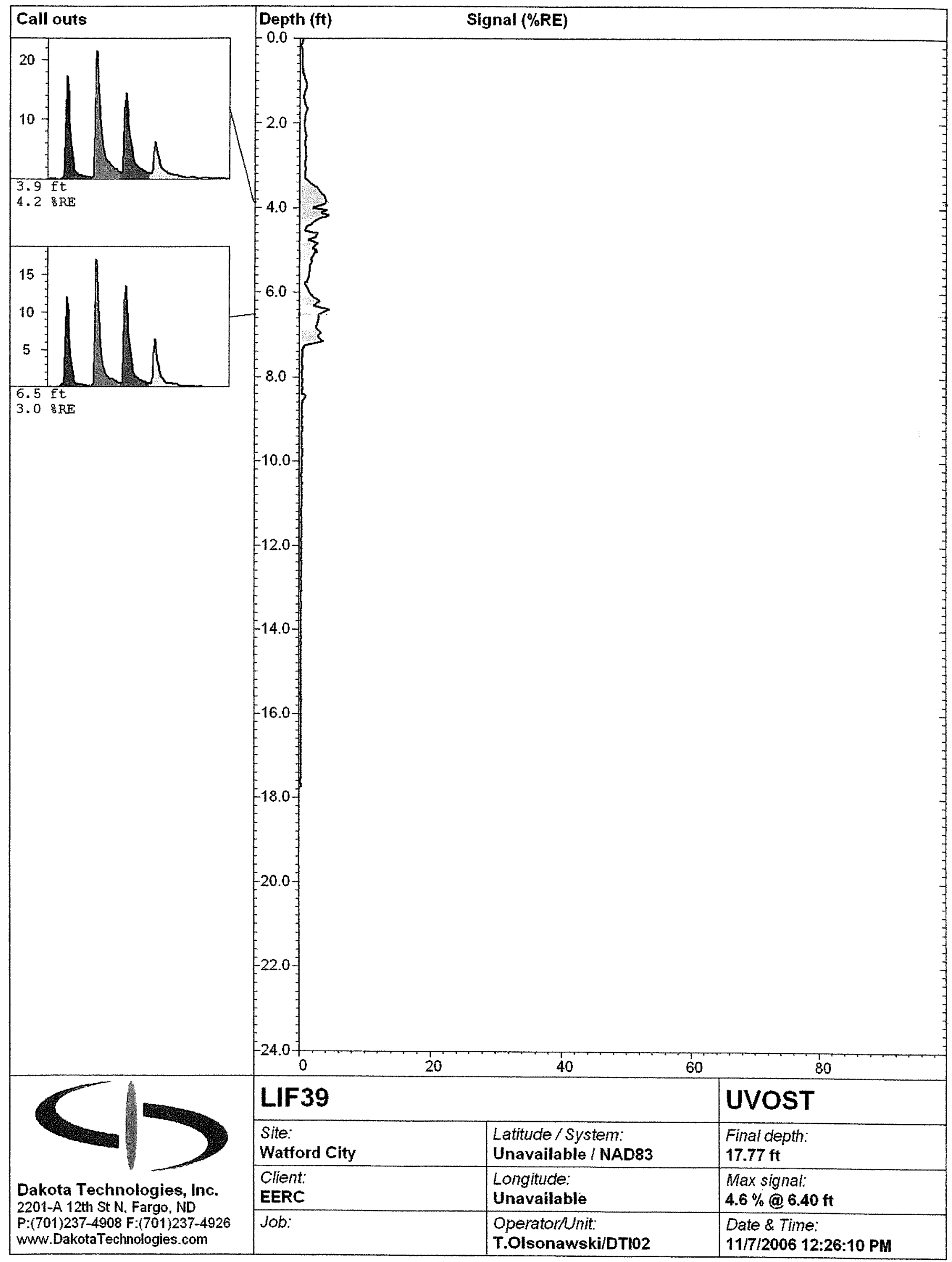




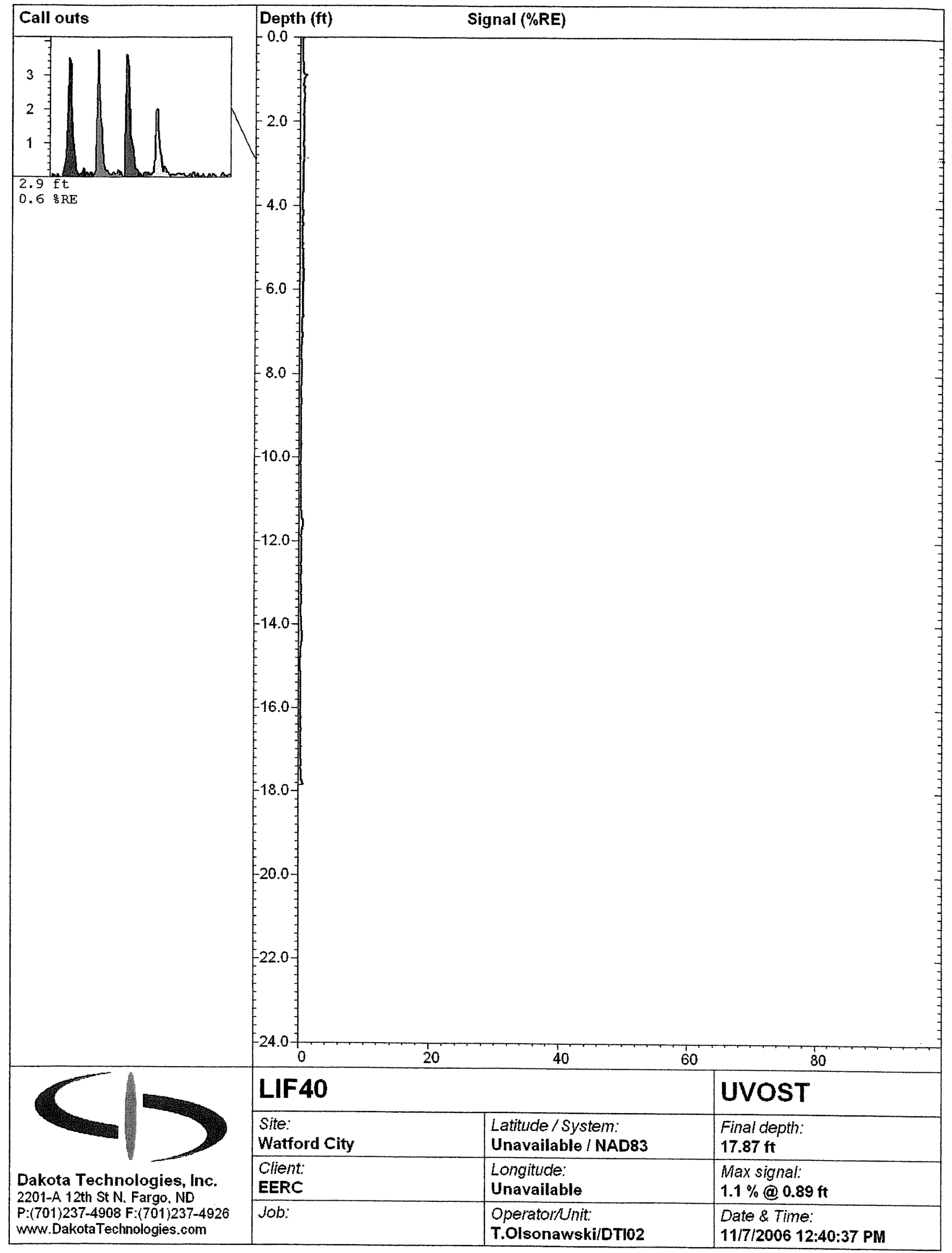




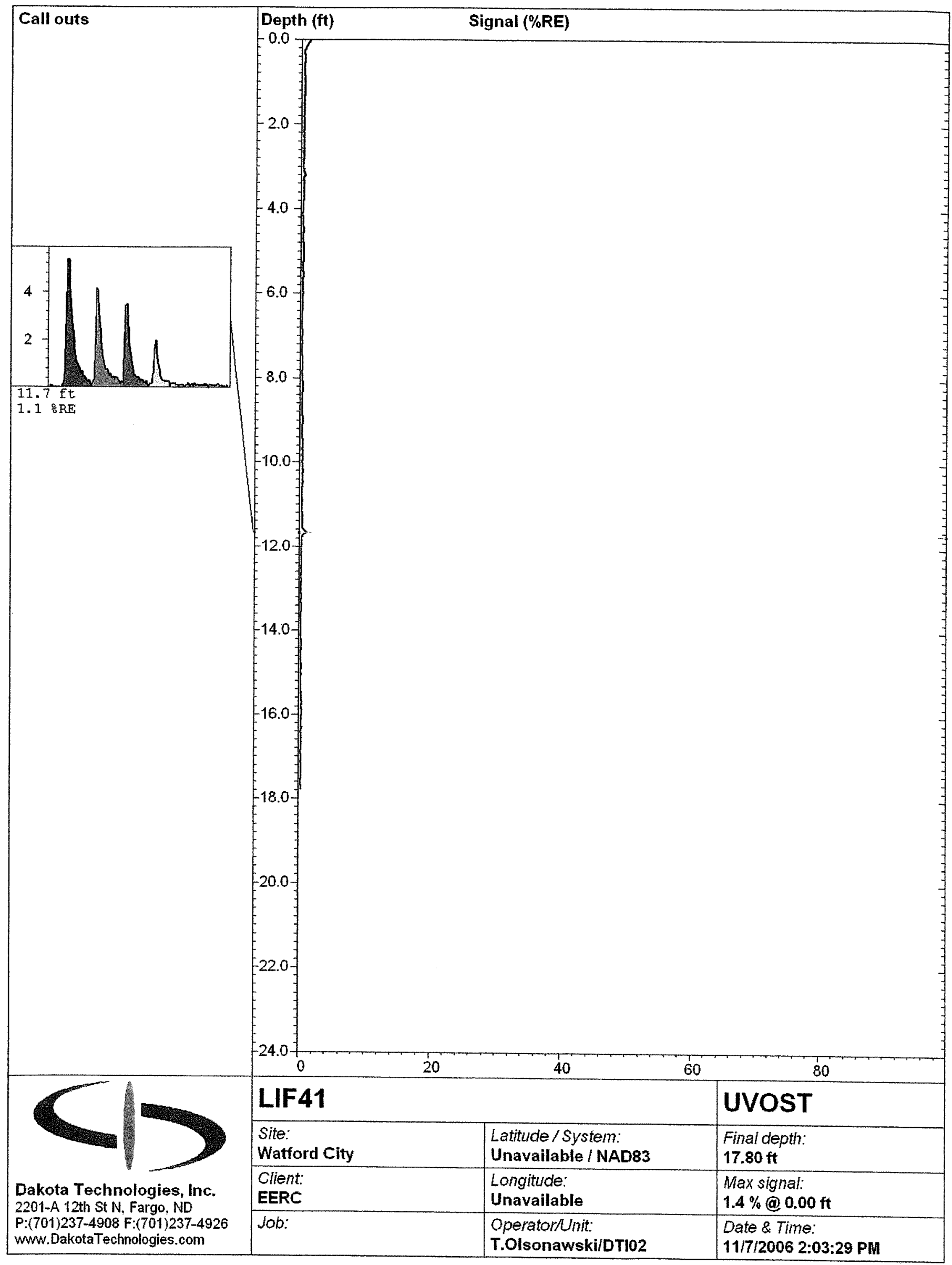




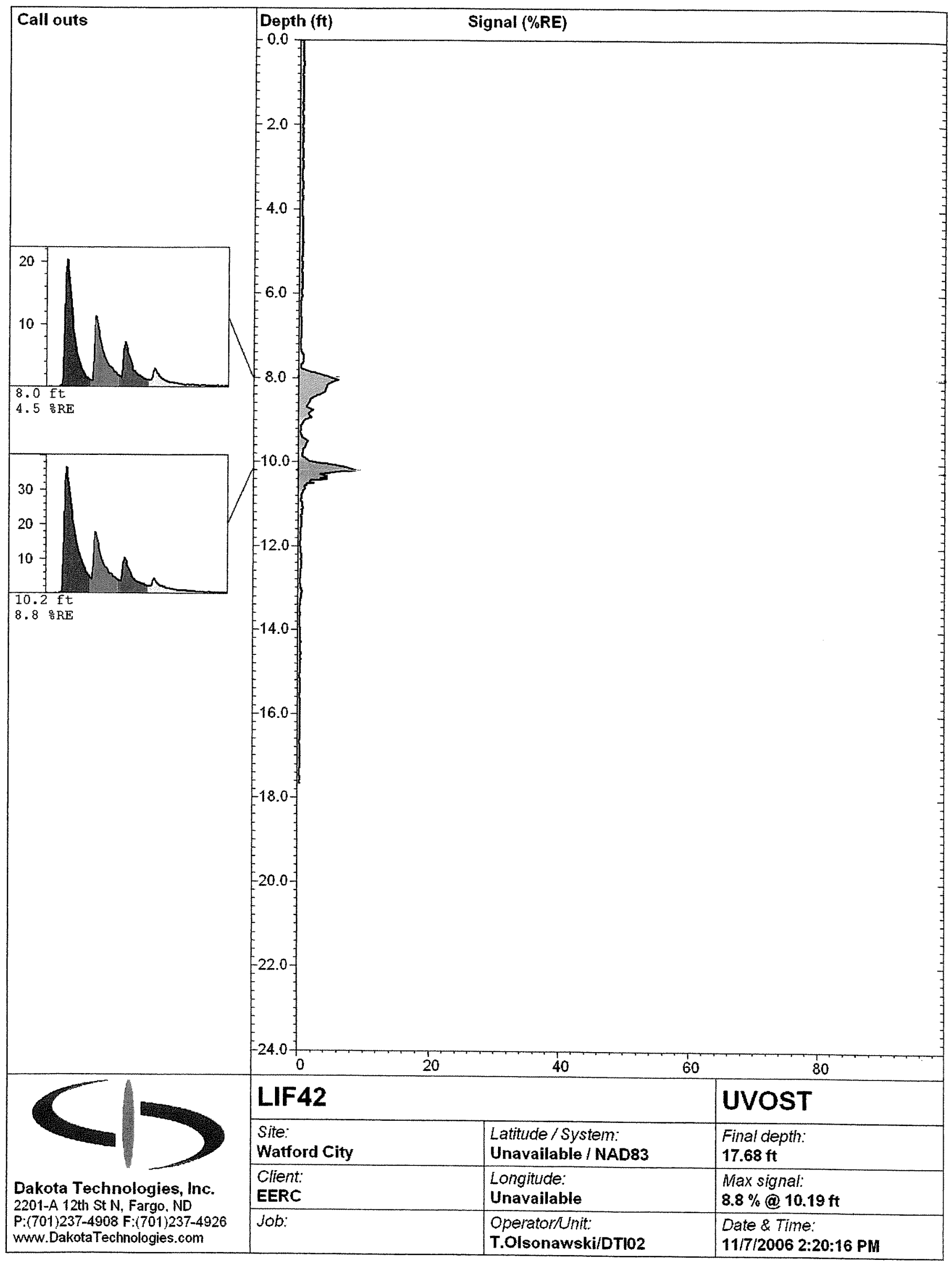




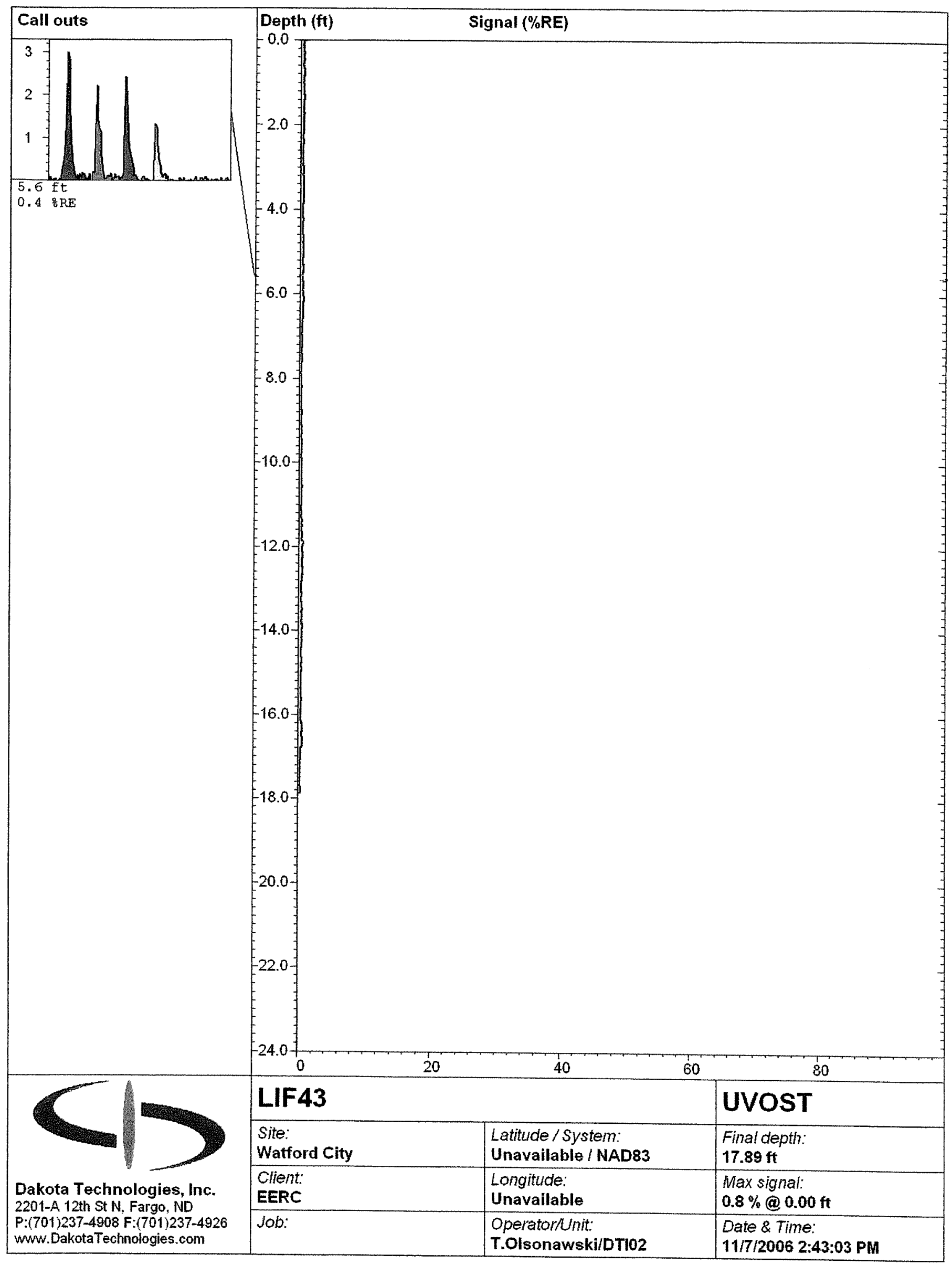




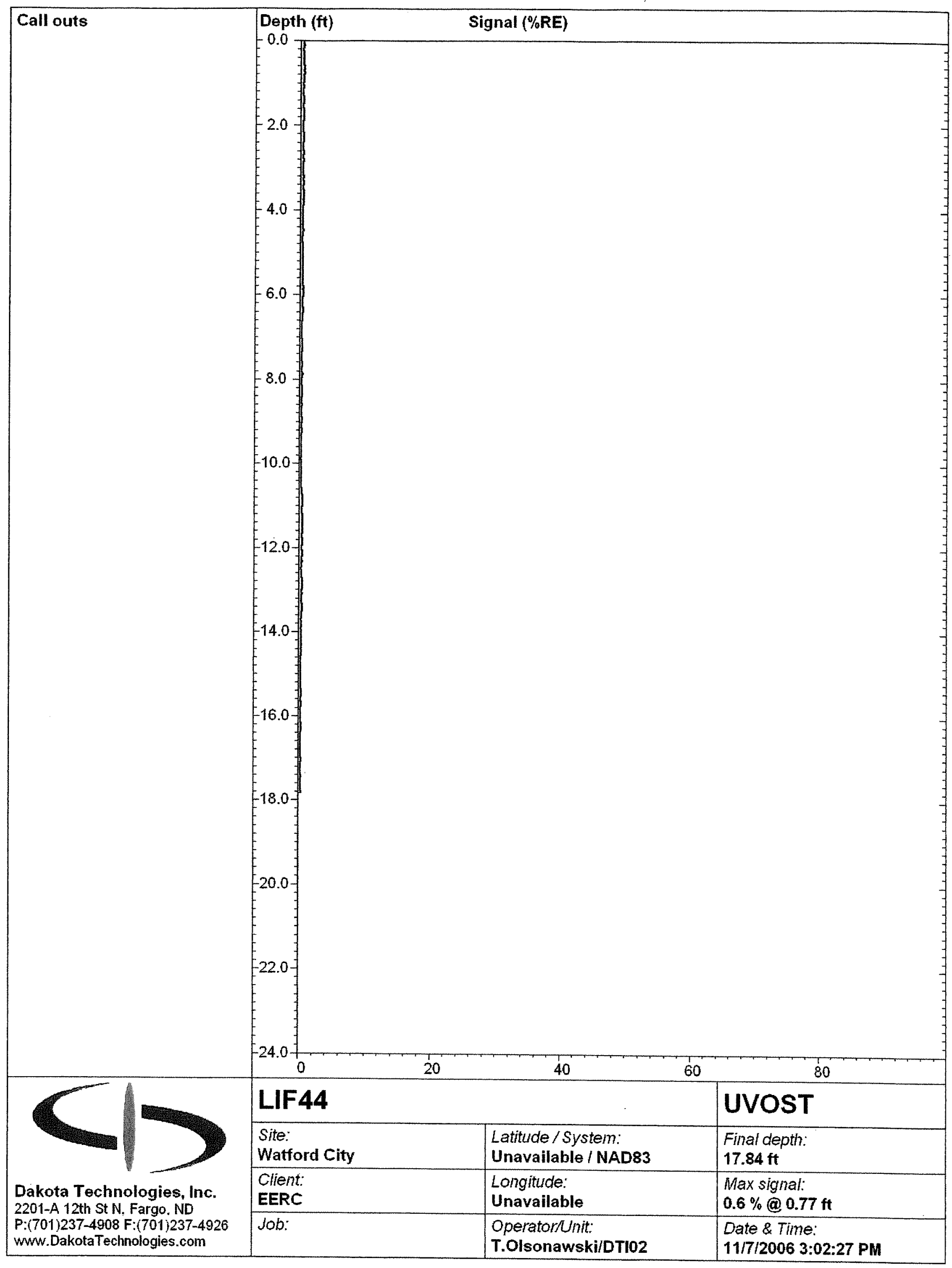




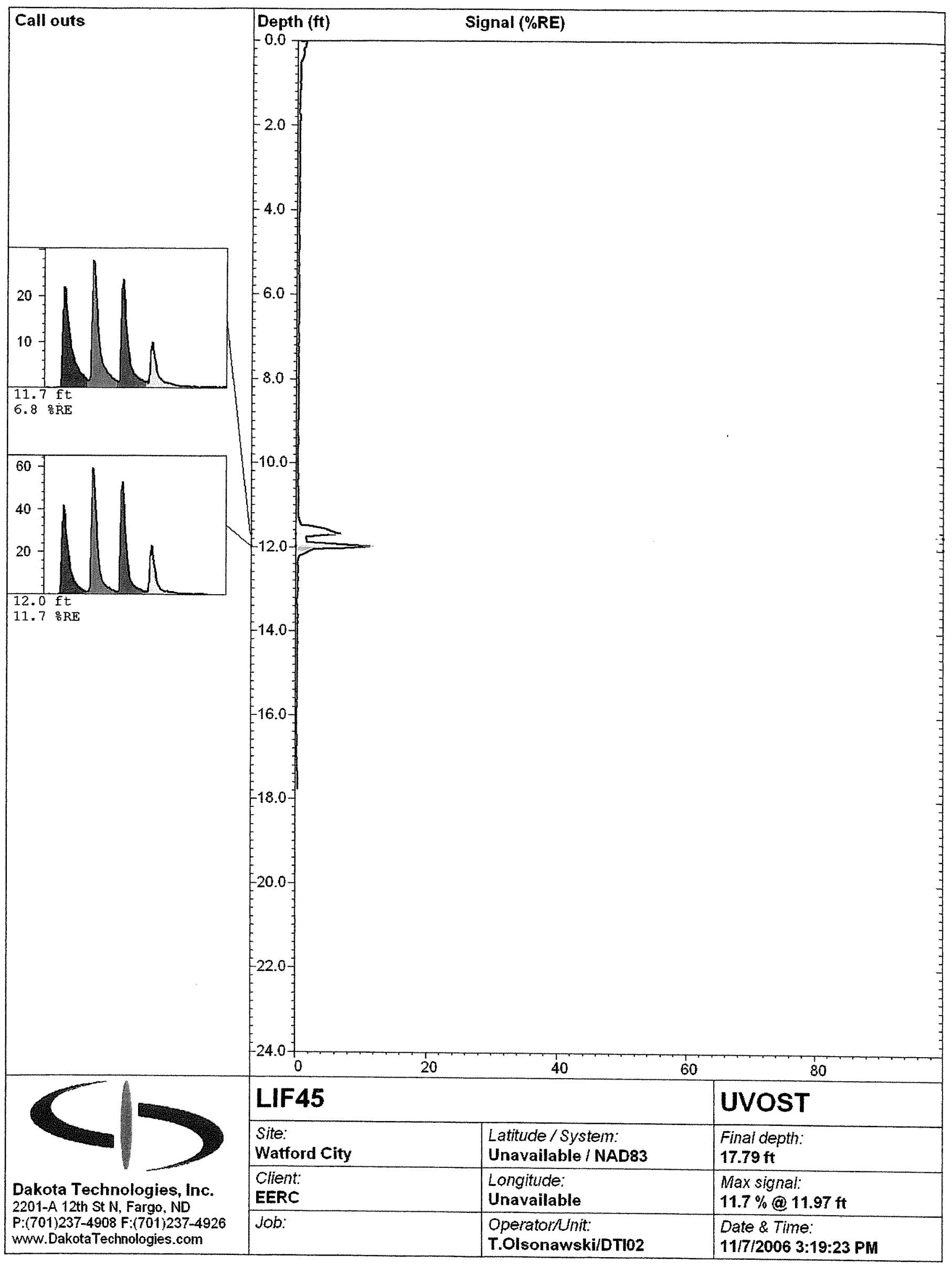




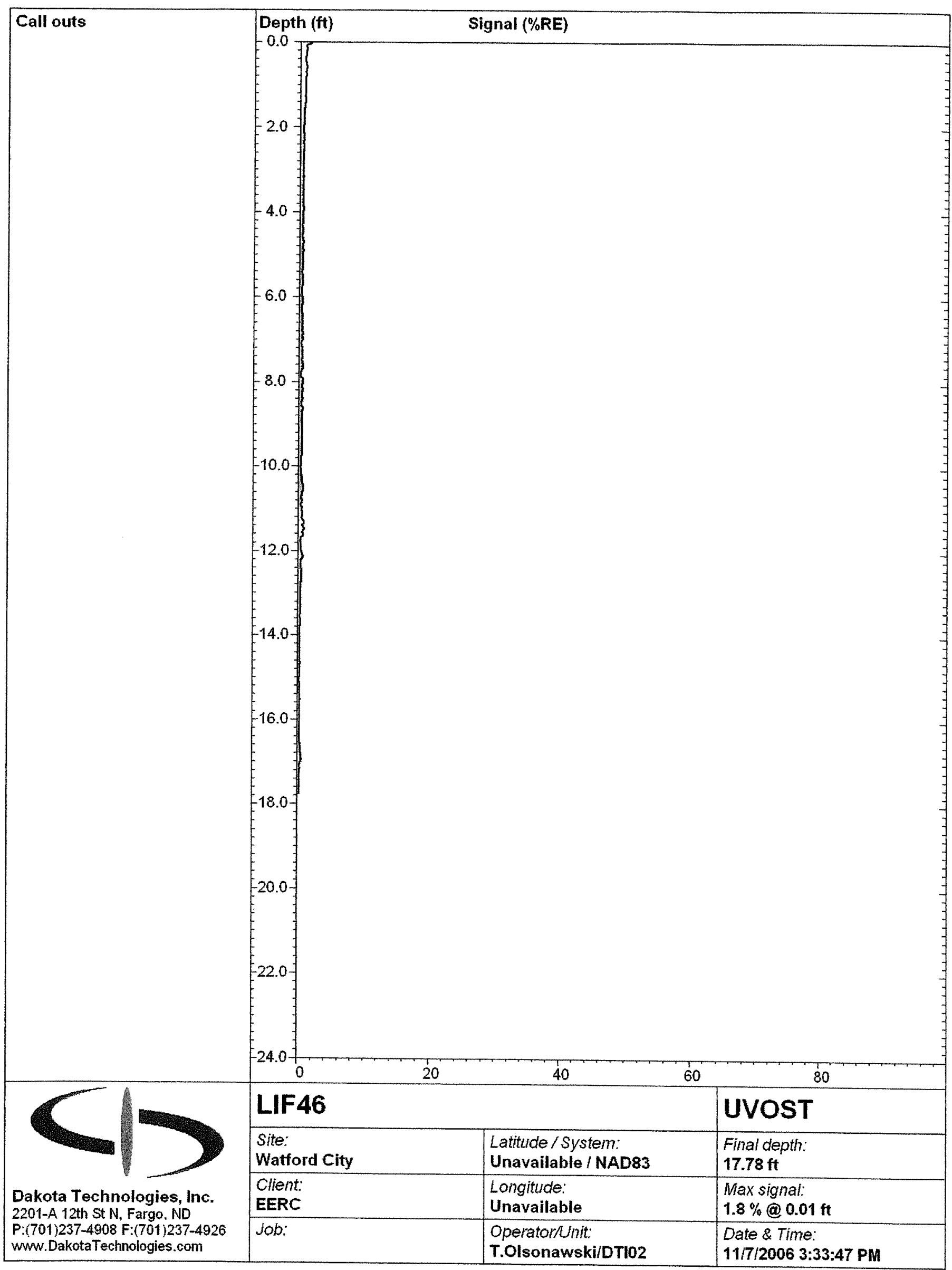


APPENDIX C

\section{GROUNDWATER TABLE MONITORING}




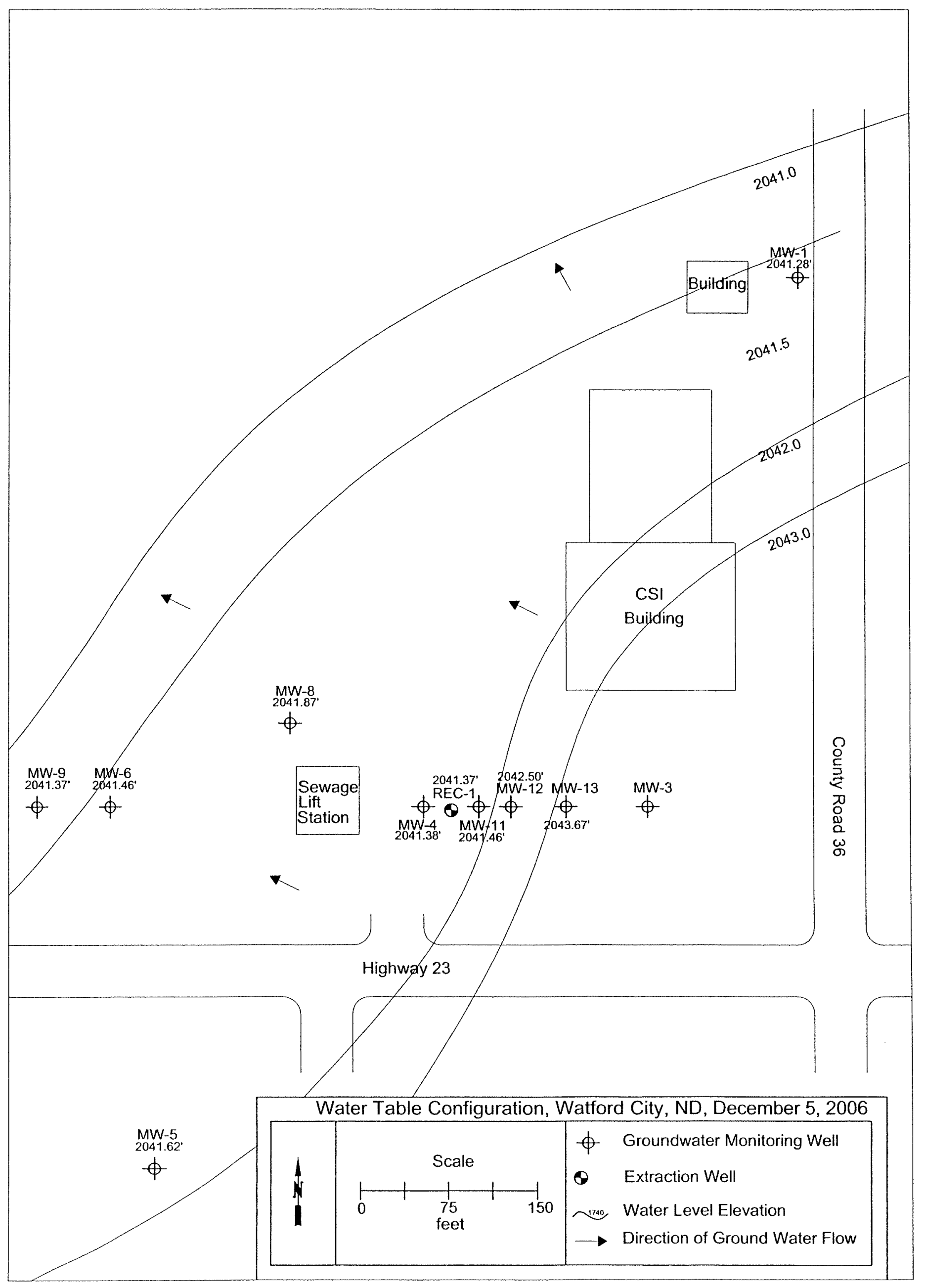




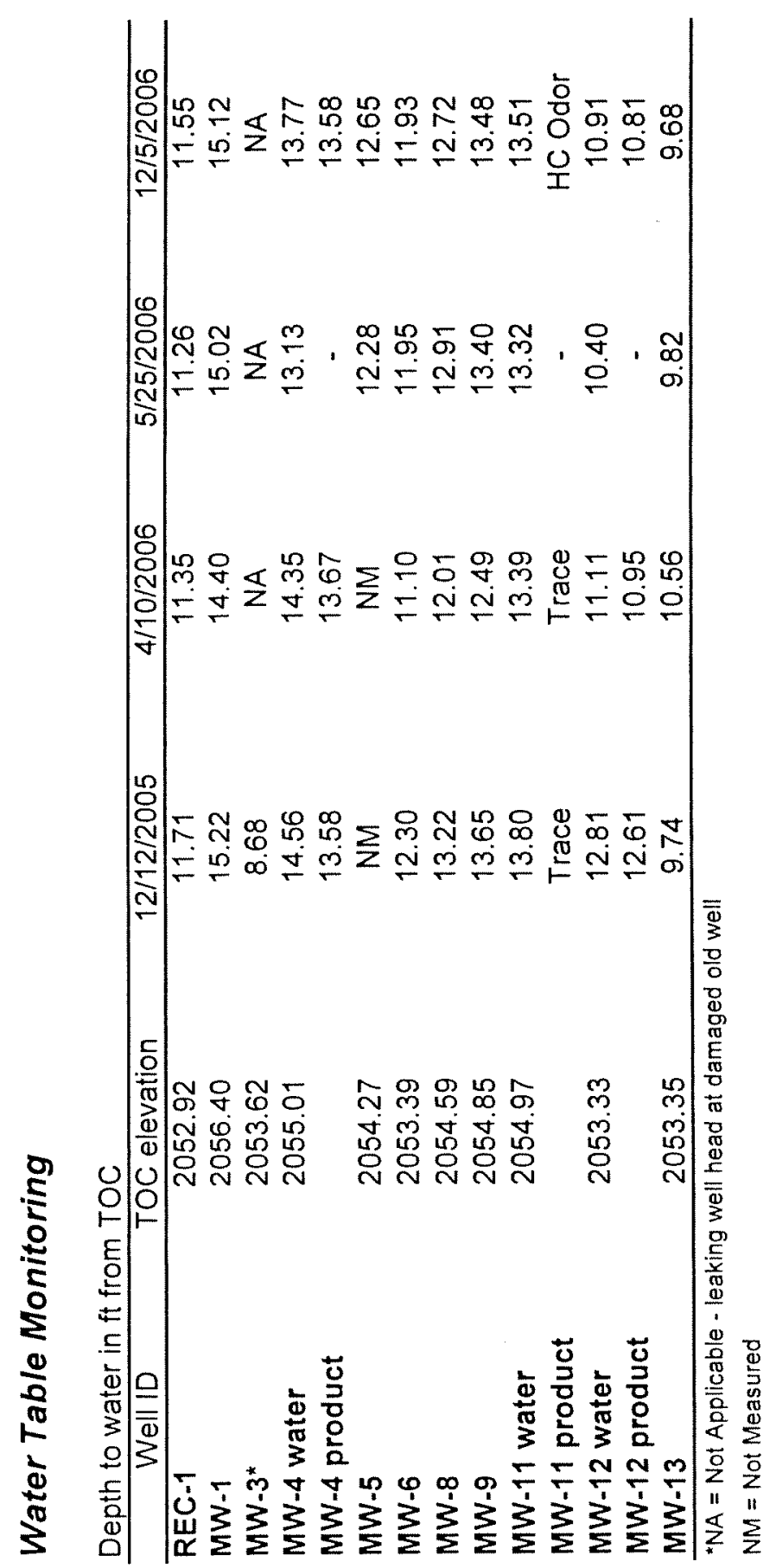




\section{APPENDIX D}

RESULTS OF HYDRAULIC TESTING 


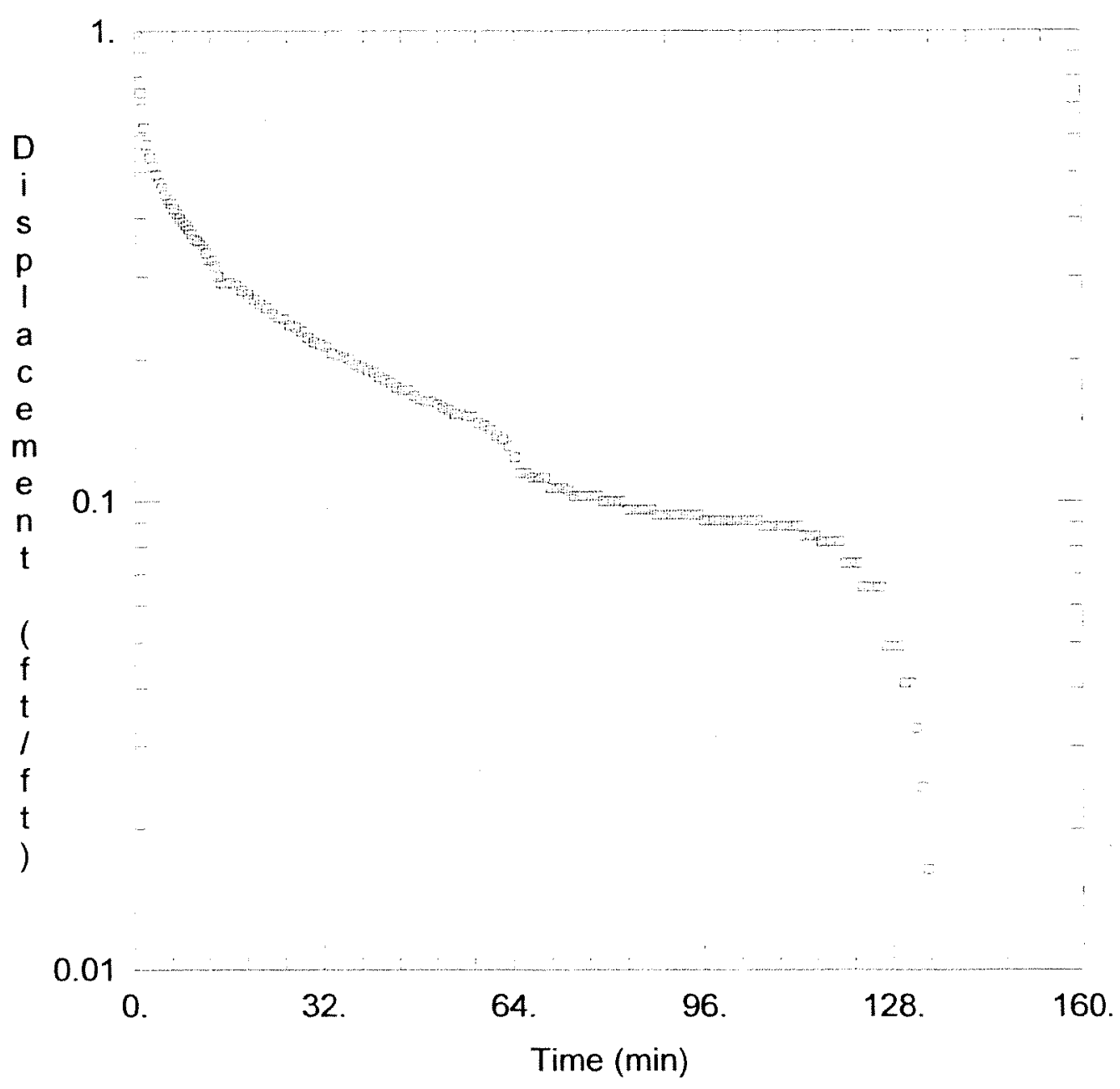

\section{SLUG TEST (SLUG REMOVAL)}

Data Set: C:IBarry FilesIIRTWWatford CitylWatford Slug TestlSlug Out full data.aqt Date: $04 / 03 / 07$ Time: $10: 20: 32$

\section{PROJECT INFORMATION}

Company: EERC

Client: NDDH

Project: Watford City

Location: North Dakota

Test Well: REC-1

Test Date: $12-5-2006$

\section{AQUIFER DATA}

Saturated Thickness: $1.5 \mathrm{ft}$

Anisotropy Ratio $(\mathrm{Kz} / \mathrm{Kr}): 1$.

\section{WELL DATA (REC-1)}

Initial Displacement: $-1.22 \mathrm{ft}$ Total Well Penetration Depth: $6.9 \mathrm{ft}$ Casing Radius: $0.17 \mathrm{ft}$
Static Water Column Height: $6.9 \mathrm{ft}$ Screen Length: $10 . \mathrm{ft}$ Wellbore Radius: $0.45 \mathrm{ft}$

\section{SOLUTION}

Aquifer Model: Unconfined

Solution Method: Bouwer-Rice

$\mathrm{K}=6.412 \mathrm{~F}-5 \mathrm{ft} / \mathrm{min}$

$v 0=-0.5882 \mathrm{ft}$ 
Data Set: C:IBarry FilesIRTIWatford CitylWatford Slug TestlSlug Out full data.aqt

Title: Slug Test (Slug Removal)

Date: $04 / 03 / 07$

Time: $10: 20: 52$

PROJECT INFORMATION

Company: EERC

Client: NDDH

Project: Watford City

Location: North Dakota

Test Date: 12-5-2006

Test Well: REC-1

\section{AQUIFER DATA}

Saturated Thickness: $1.5 \mathrm{ft}$

Anisotropy Ratio (Kz/Kr): 1.

\section{SLUG TEST WELL DATA}

Test Well: : REC-1

$X$ Location: $0 . \mathrm{ft}$

$Y$ Location: $0 . \mathrm{ft}$

Initial Displacement: $-1.22 \mathrm{ft}$

Static Water Column Height: $6.9 \mathrm{ft}$

Casing Radius: $0.17 \mathrm{ft}$

Wellbore Radius: $0.45 \mathrm{ft}$

Well Skin Radius: $0.6 \mathrm{ft}$

Screen Length: $10 . \mathrm{ft}$

Total Well Penetration Depth: $6.9 \mathrm{ft}$

No. of Observations: 151

\begin{tabular}{cccc} 
& \multicolumn{2}{c}{ Observation Data } & \\
Time $(\mathrm{min})$ & Displacement $(\mathrm{ft})$ & Time $(\mathrm{min})$ & Displacement $(\mathrm{ft})$ \\
\hline 0. & 0. & 61. & -0.168 \\
0.5 & -1.214 & 62. & -0.1651 \\
1. & -0.9014 & 63. & -0.1595 \\
1.5 & -0.7606 & 64. & -0.151 \\
2. & -0.6986 & 65. & -0.14 \\
2.5 & -0.6564 & 66. & -0.14 \\
3. & -0.6282 & 67. & -0.137 \\
3.5 & -0.6028 & 68. & -0.137 \\
4. & -0.5803 & 69. & -0.137 \\
4.5 & -0.5662 & 70. & -0.13 \\
5. & -0.5465 & 71. & -0.13 \\
5.5 & -0.5352 & 72. & -0.13 \\
6. & -0.524 & 73. & -0.1285 \\
6.5 & -0.5099 & 74. & -0.125 \\
7. & -0.4986 & 75. & -0.125 \\
7.5 & -0.4873 & 76. & -0.125
\end{tabular}




\begin{tabular}{|c|c|c|c|}
\hline Time (min) & Displacement (ft) & Time (min) & Displacement (ft) \\
\hline 8 & -0.4789 & 77. & -0.125 \\
\hline 8.5 & -0.4704 & 78. & -0.125 \\
\hline 9. & -0.462 & 79 . & -0.122 \\
\hline 9.5 & -0.4507 & 80. & -0.122 \\
\hline 10. & -0.4395 & 81. & -0.122 \\
\hline 10.5 & -0.4366 & 82. & -0.122 \\
\hline 11. & -0.4282 & 83. & -0.117 \\
\hline 11.5 & -0.4197 & 84. & -0.117 \\
\hline 12. & -0.4113 & 85. & -0.117 \\
\hline 12.5 & -0.3972 & 86. & -0.117 \\
\hline 13. & -0.3972 & 87. & -0.117 \\
\hline 13.5 & -0.3859 & 88. & -0.114 \\
\hline 14. & -0.3662 & 89. & -0.114 \\
\hline 14.5 & -0.355 & 90. & -0.114 \\
\hline 15. & -0.3521 & 91. & -0.114 \\
\hline 16. & -0.356 & 92. & -0.114 \\
\hline 17. & -0.351 & 93. & -0.114 \\
\hline 18. & -0.342 & 94. & -0.114 \\
\hline 19. & -0.336 & 95. & -0.114 \\
\hline 20. & -0.328 & 96. & -0.111 \\
\hline 21. & -0.32 & 97. & -0.111 \\
\hline 22. & -0.314 & 98. & -0.111 \\
\hline 23. & -0.305 & 99. & -0.111 \\
\hline 24. & -0.3 & 100. & -0.111 \\
\hline 25. & -0.2975 & 101. & -0.111 \\
\hline 26. & -0.289 & 102. & -0.111 \\
\hline 27. & -0.2862 & 103. & -0.111 \\
\hline 28. & -0.28 & 104. & -0.111 \\
\hline 29. & -0.272 & 105. & -0.111 \\
\hline 30. & -0.266 & 106. & -0.108 \\
\hline 31. & -0.263 & 107. & -0.108 \\
\hline 32. & -0.26 & 108. & -0.108 \\
\hline 33. & -0.252 & 109. & -0.108 \\
\hline 34. & -0.249 & 110. & -0.108 \\
\hline 35. & -0.2468 & 111. & -0.108 \\
\hline 36. & -0.244 & 112. & -0.108 \\
\hline 37. & -0.238 & 113. & -0.103 \\
\hline 38. & -0.235 & 114. & -0.103 \\
\hline 39. & -0.232 & 115. & -0.103 \\
\hline 40. & -0.229 & 116. & -0.1 \\
\hline 41. & -0.224 & 117. & -0.1 \\
\hline 42. & -0.221 & 118. & -0.1 \\
\hline 43. & -0.218 & 119. & -0.1 \\
\hline 44. & -0.213 & 120. & -0.09 \\
\hline 45. & -0.21 & 121. & -0.09 \\
\hline 46. & -0.21 & 122. & -0.09 \\
\hline 47. & -0.2045 & 123. & -0.08 \\
\hline 48. & -0.201 & 124. & -0.08 \\
\hline 49. & -0.1989 & 125. & -0.08 \\
\hline 50. & -0.1989 & 126. & -0.08 \\
\hline 51. & -0.196 & 127. & -0.06 \\
\hline 52. & -0.193 & 128. & -0.06 \\
\hline 53. & -0.1905 & 129. & -0.06 \\
\hline
\end{tabular}




\begin{tabular}{cccc} 
Time $(\mathrm{min})$ & Displacement $(\mathrm{ft})$ & Time $(\mathrm{min})$ & Displacement $(\mathrm{ft})$ \\
\hline 54. & -0.187 & 130. & -0.05 \\
55. & -0.187 & 131. & -0.05 \\
56. & -0.1848 & 132. & -0.04 \\
57. & -0.1848 & 133. & -0.03 \\
58. & -0.179 & 134. & -0.02 \\
59. & -0.1764 & 135. & -0.01 \\
60. & -0.173 & &
\end{tabular}

\section{SOLUTION}

Aquifer Model: Unconfined

Solution Method: Bouwer-Rice

Shape Factor: 2.08

\section{VISUAL ESTIMATION RESULTS}

Estimated Parameters

$\begin{array}{ccl}\text { Parameter } & \text { Estimate } & \\ \mathrm{K} & 6.439 \mathrm{E}-5 & \mathrm{ft} / \mathrm{min} \\ \mathrm{y} 0 & -0.5888 \mathrm{ft}\end{array}$

\section{AUTOMATIC ESTIMATION RESULTS}

\section{Estimated Parameters}

\begin{tabular}{cccc} 
Parameter & Estimate & Std. Error & \\
\cline { 2 - 2 } & $6.412 \mathrm{E}-5$ & $3.755 \mathrm{E}-6$ & $\mathrm{ft} / \mathrm{min}$ \\
$\mathrm{y} 0$ & -0.5882 & $0.01936 \mathrm{ft}$
\end{tabular}

Parameter Correlations

$\begin{array}{ccc}\mathrm{K} & \mathrm{K} & \mathrm{y0} \\ \mathrm{y} 0 & -0.00 & -0.66 \\ 1.00\end{array}$

Residual Statistics

for weighted residuals

Sum of Squares .. $1.097 \mathrm{ft}^{2}$

Variance ......... $0.007365 \mathrm{ft}^{2}$

Std. Deviation..... $0.08582 \mathrm{ft}$

Mean............ $-0.00744 \mathrm{ft}$

No. of Residuals .. 151

No. of Estimates .. 2 
Will locates be done, do we have permission to drill in all quadrants?

Do businesses on-site know that we're coming?

Meet drillers at what time and where, names, phone numbers of drillers.

Location of wells? Number of wells? Type of wells (still 14 " and 42 "?)

What is mission? If delineation, then spread wells out to sides. If to prepare for hydro test, then that's another matter. It's tough to focus on both, you may get incidental results by shooting for hydro focus.

How deep into the water table do you need the wells for hydro test? How much screen above/below water table? Completion of wells.

Assuming groundwater is at 20 feet (average around site), what length of screen for each type of well and where do you want the water level to fall (what is the target interval)?

Also, are we using standard completion methods: sand to two feet over screen then bentonite chips to surface? Then a flush mount? Will they be locked?

Are other wells existing on-site locked, if so can I get the keys to take water levels?

Are we sampling on the way down? If so, what kind, split spoons or simply from auger flights? Is it just baggie/PID sampling? If we are sampling with split spoons, what interval (continuous/ every 5-feet)? Do drillers have DECON equipment for spoons/augers?

If collecting samples for lab, get bottle requirements and all lab information.

Take 40 -hour refresher.

Make reservation at Raddison, get truck reserved

\section{EOUIPMENT}

Drill logs

Rubber gloves

Digital Camera

PID Calibration gas

Baggies

Hard hat

Safery Glasses

Markers/Pens/pencils

Ficld Book

Work boots

Water level indicator/Product indicator

Decon stuff??

Soil classification information from $A K$.

Munsel Soil color Book

Copies of all previous work and workplans

Copy of drill permit that Jarda was talking about.
5- METHANE CYLINDCRS

Bat cAMPBacl (Evoring)

S- cavister (bimit)

Z- tediar bags

Bliti Bpina

Fioure 1

- N. Arrón

- SCALE 
1.

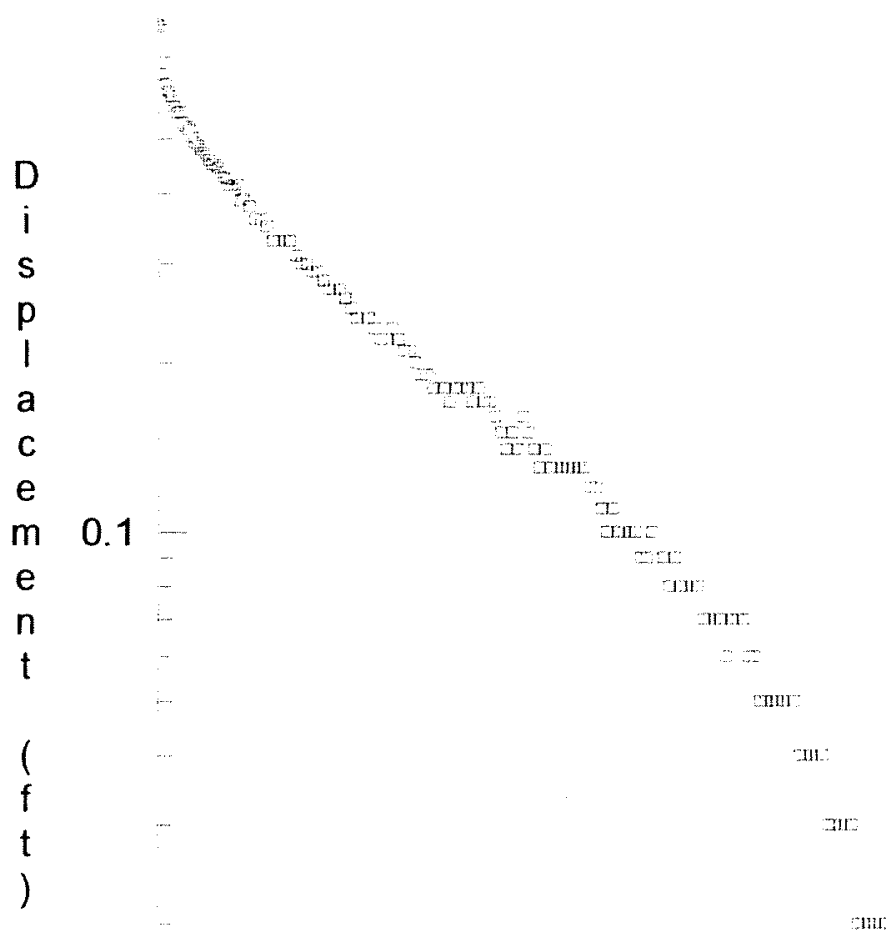

0.01

0.

40.

80.

120.

160.

200.

Time $(\min )$

SLUG TEST (SLUG IN)

Data Set: C:IBarry FilesIIRTIWatford CitylWatford Slug TestlAqtw2.aqt

Date: $04 / 02 / 07$

Time: $12: 36: 39$

\section{PROJECT INFORMATION}

Company: EERC

Client: NDDH

Project: Watford City

Location: North Dakota

Test Well: REC-1

Test Date: 12-5-2006

Saturated Thickness: $0.5 \mathrm{ft}$

\section{AQUIFER DATA}

Anisotropy Ratio (Kz/Kr): 1.

WELL DATA (REC-1)

Initial Displacement: $0.8 \mathrm{ft}$ Total Well Penetration Depth: $6.9 \mathrm{ft}$ Static Water Column Height: $6.9 \mathrm{ft}$ Screen Length: $10 . \mathrm{ft}$

Casing Radius: $0.17 \mathrm{ft}$ Wellbore Radius: $0.45 \mathrm{ft}$

\section{SOLUTION}

Aquifer Model: Unconfined

Solution Method: Bouwer-Rice

$\mathrm{K}=6701 \mathrm{~F}-5 \mathrm{ft} / \mathrm{min}$

$v n=0.5623 \mathrm{ft}$ 
Data Set: C:IBarry Files IRTIWatford CitylWatford Slug TestlAqtw2.aqt

Title: Slug Test (Slug In)

Date: $04 / 02 / 07$

Time: $12: 37: 04$

PROJECT INFORMATION

Company: EERC

Client: NDDH

Project: Watford City

Location: North Dakota

Test Date: 12-5-2006

Test Well: REC-1

\section{AQUIFER DATA}

Saturated Thickness: $0.5 \mathrm{ft}$

Anisotropy Ratio $(\mathrm{Kz} / \mathrm{Kr}): 1$.

\section{SLUG TEST WELL DATA}

Test Well: : REC-1

$X$ Location: $0 . \mathrm{ft}$

Y Location: $0 . \mathrm{ft}$

Initial Displacement: $0.8 \mathrm{ft}$

Static Water Column Height: $6.9 \mathrm{ft}$

Casing Radius: $0.17 \mathrm{ft}$

Wellbore Radius: $0.45 \mathrm{ft}$

Well Skin Radius: $0.6 \mathrm{ft}$

Screen Length: 10. ft

Total Well Penetration Depth: $6.9 \mathrm{ft}$

No. of Observations: 150

\begin{tabular}{cccc} 
& \multicolumn{2}{c}{ Observation Data } & \\
Time $(\mathrm{min})$ & Displacement $(\mathrm{ft})$ & Time $(\mathrm{min})$ & Displacement $(\mathrm{ft})$ \\
\cline { 2 - 3 } 0. & 0. & 60. & 0.15 \\
0.5 & 0.7943 & 61. & 0.14 \\
1. & 0.6535 & 62. & 0.15 \\
1.5 & 0.6281 & 63. & 0.14 \\
2. & 0.6084 & 64. & 0.16 \\
2.5 & 0.5971 & 65. & 0.15 \\
3. & 0.5718 & 66. & 0.14 \\
3.5 & 0.5577 & 67. & 0.13 \\
4. & 0.5605 & 68. & 0.14 \\
4.5 & 0.5352 & 69. & 0.13 \\
5. & 0.5267 & 70. & 0.13 \\
5.5 & 0.5211 & 71. & 0.13 \\
6. & 0.5042 & 72. & 0.13 \\
6.5 & 0.4929 & 73. & 0.13 \\
7. & 0.4873 & 74. & 0.13 \\
7.5 & 0.4788 & 75. & 0.13
\end{tabular}




\begin{tabular}{|c|c|c|c|}
\hline Time (min) & Displacement (ft) & Time (min) & Displacement (ft) \\
\hline 8. & 0.4732 & 76. & 0.12 \\
\hline 8.5 & 0.4676 & 77. & 0.12 \\
\hline 9. & 0.4591 & 78. & 0.11 \\
\hline 9.5 & 0.4507 & 79. & 0.1 \\
\hline 10. & 0.4507 & 80. & 0.11 \\
\hline 10.5 & 0.445 & 81. & 0.1 \\
\hline 11. & 0.4366 & 82. & 0.1 \\
\hline 11.5 & 0.4281 & 83. & 0.1 \\
\hline 12. & 0.4253 & 84. & 0.1 \\
\hline 12.5 & 0.4141 & 85. & 0.09 \\
\hline 13. & 0.4141 & 86. & 0.09 \\
\hline 13.5 & 0.4084 & 87. & 0.1 \\
\hline 14 & 0.4141 & 89. & 0.09 \\
\hline 14.5 & 0.3887 & 90. & 0.08 \\
\hline 15. & 0.3915 & 91. & 0.09 \\
\hline 16. & 0.38 & 92. & 0.08 \\
\hline 17. & 0.36 & 93. & 0.08 \\
\hline 18. & 0.36 & 94. & 0.08 \\
\hline 19. & 0.35 & 95. & 0.08 \\
\hline 20. & 0.33 & 96. & 0.07 \\
\hline 21. & 0.33 & 97. & 0.07 \\
\hline 22. & 0.33 & 98. & 0.07 \\
\hline 23. & 0.33 & 99. & 0.07 \\
\hline 24. & 0.31 & 100. & 0.06 \\
\hline 25. & 0.3 & 101. & 0.07 \\
\hline 26. & 0.299 & 102. & 0.07 \\
\hline 27. & 0.29 & 103. & 0.07 \\
\hline 28. & 0.29 & 104. & 0.06 \\
\hline 29. & 0.28 & 105. & 0.06 \\
\hline 30. & 0.27 & 106. & 0.05 \\
\hline 31. & 0.27 & 107. & 0.05 \\
\hline 32. & 0.27 & 108. & 0.05 \\
\hline 33. & 0.26 & 109. & 0.05 \\
\hline 34. & 0.25 & 110. & 0.05 \\
\hline 35. & 0.24 & 111. & 0.05 \\
\hline 36. & 0.24 & 112. & 0.05 \\
\hline 37. & 0.24 & 113. & 0.04 \\
\hline 38. & 0.23 & 114. & 0.04 \\
\hline 39. & 0.22 & 115. & 0.04 \\
\hline 40. & 0.22 & 116. & 0.04 \\
\hline 41. & 0.23 & 117. & 0.04 \\
\hline 42. & 0.22 & 118. & 0.03 \\
\hline 43. & 0.21 & 119. & 0.03 \\
\hline 44. & 0.21 & 120. & 0.03 \\
\hline 45. & 0.2 & 121. & 0.03 \\
\hline 46. & 0.19 & 122. & 0.03 \\
\hline 47. & 0.19 & 123. & 0.02 \\
\hline 48. & 0.18 & 124. & 0.02 \\
\hline 49. & 0.18 & 125. & 0.02 \\
\hline 50. & 0.18 & 126. & 0.02 \\
\hline 51. & 0.17 & 127. & 0.02 \\
\hline 52. & 0.18 & 128. & 0.02 \\
\hline 53. & 0.18 & 129. & 0.01 \\
\hline
\end{tabular}




$\begin{array}{cccc}\text { Time }(\mathrm{min}) & \text { Displacement }(\mathrm{ft}) & \text { Time }(\mathrm{min}) & \text { Displacement }(\mathrm{ft}) \\ 54 . & 0.18 & 130 . & 0.01 \\ 55 . & 0.17 & 131 . & 0.01 \\ 56 . & 0.18 & 132 . & 0.01 \\ 57 . & 0.17 & 133 . & 0.01 \\ 58 . & 0.17 & 134 . & 0.01 \\ 59 . & 0.16 & 135 . & 0 .\end{array}$

SOLUTION

Aquifer Model: Unconfined

Solution Method: Bouwer-Rice

Shape Factor: 2.08

VISUAL ESTIMATION RESULTS

Estimated Parameters

$\begin{array}{ccl}\text { Parameter } & \text { Estimate } & \\ \text { K } & 6.701 \mathrm{E}-5 & \mathrm{ft} / \mathrm{min} \\ \mathrm{y} 0 & 0.5623 \mathrm{ft}\end{array}$




\section{APPENDIX E}

WELLHEAD PROTECTION AREA 


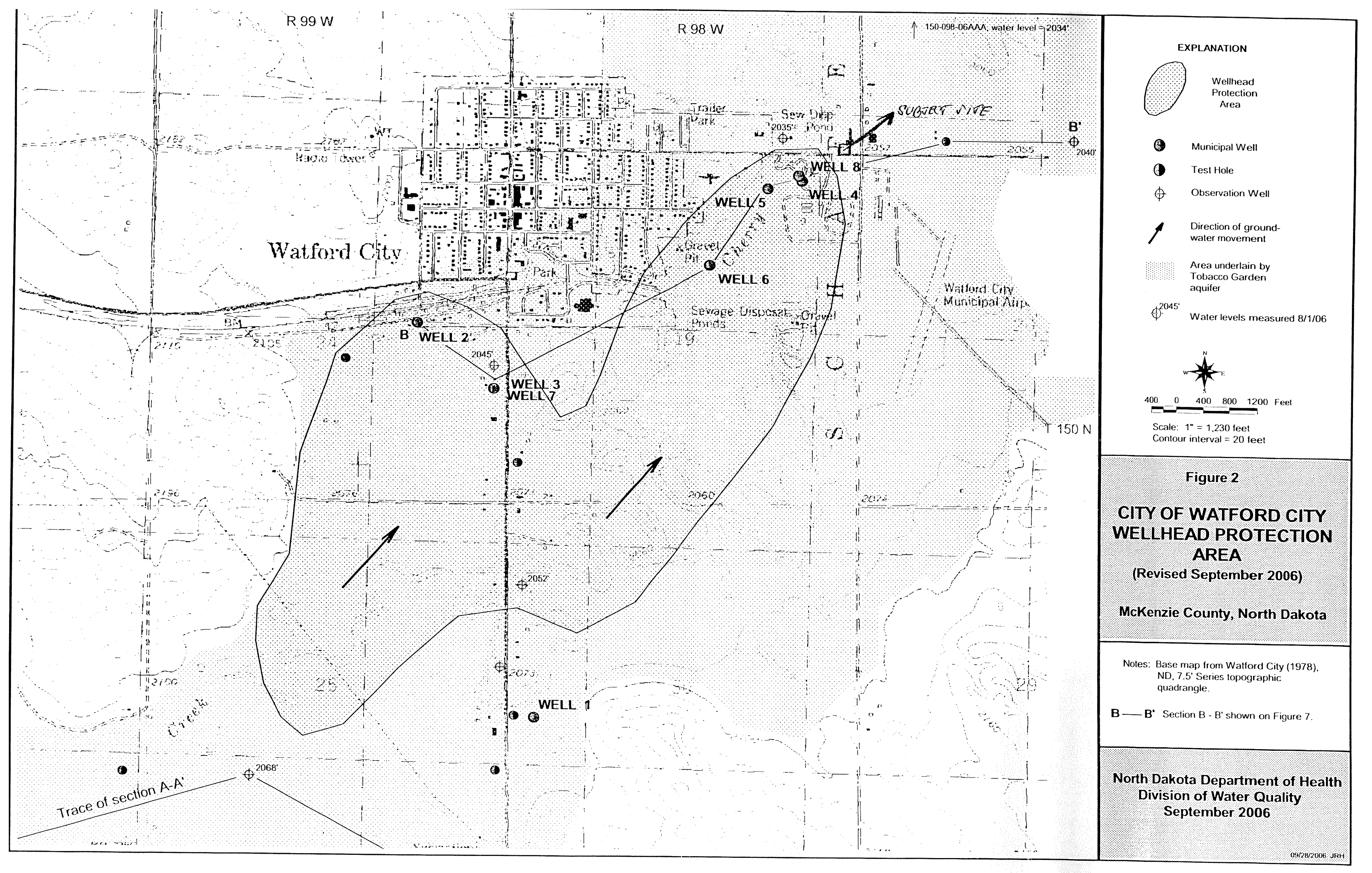


APPENDIX F

SUMMARY OF ANALYTICAL DATA 


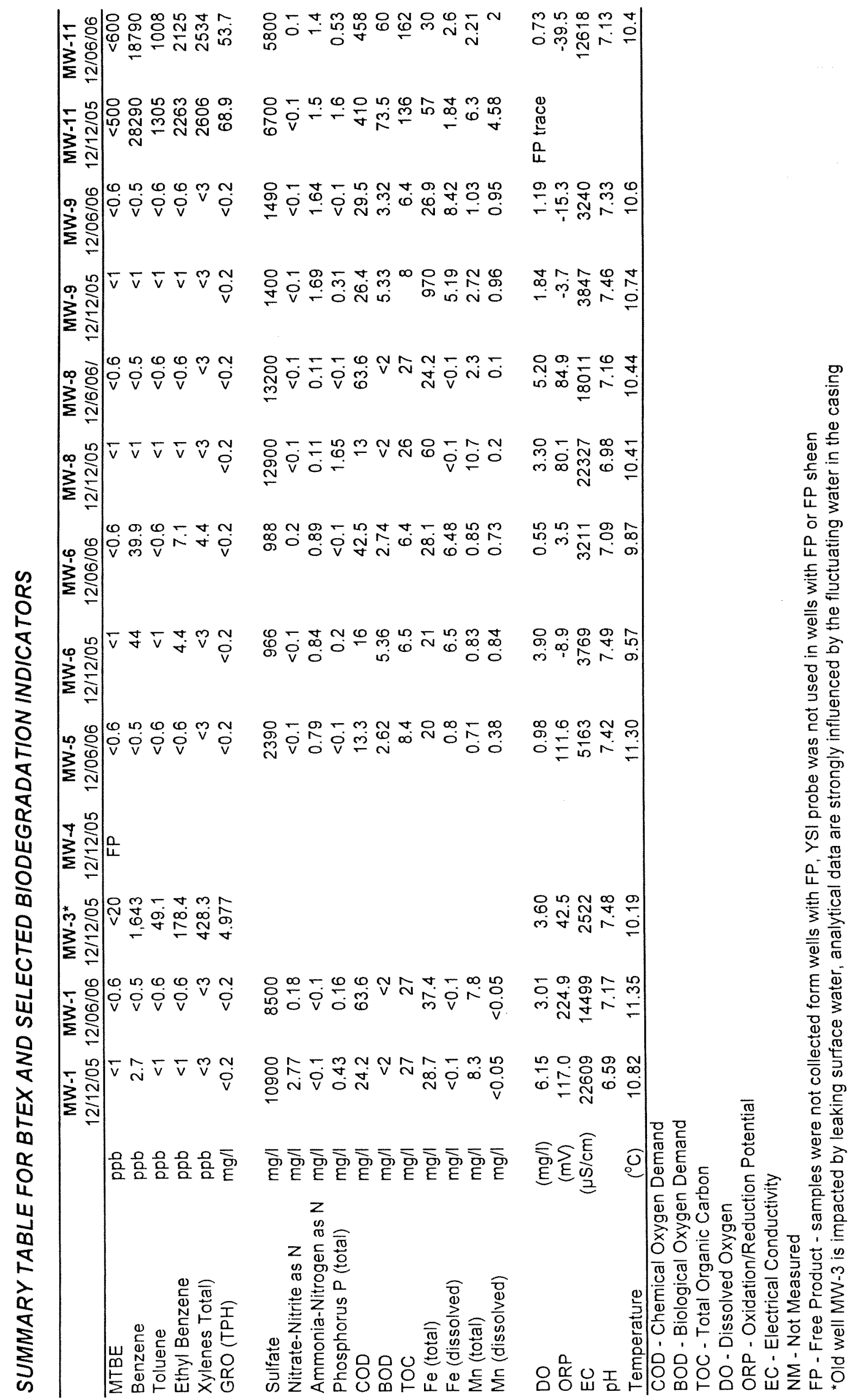




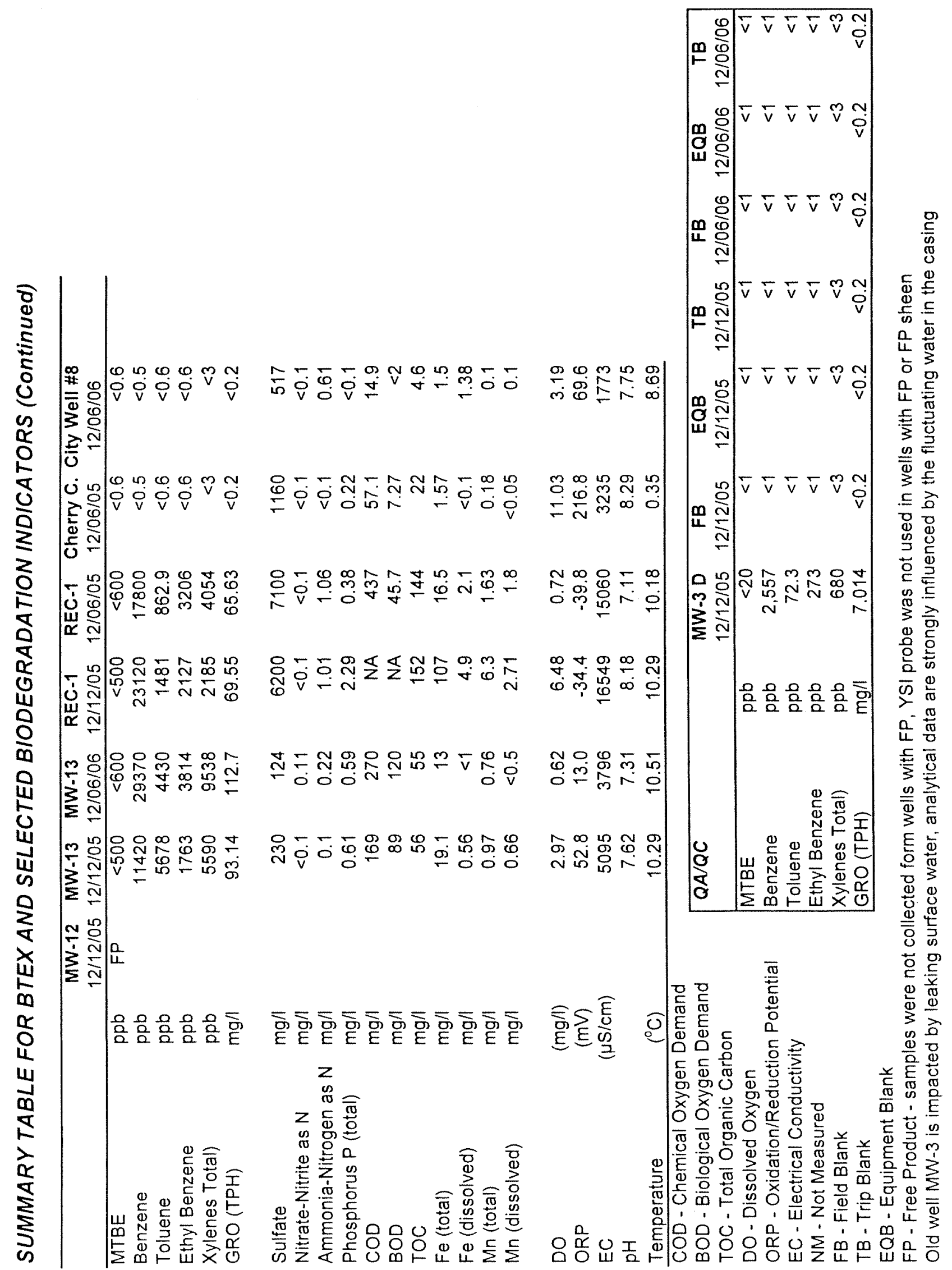




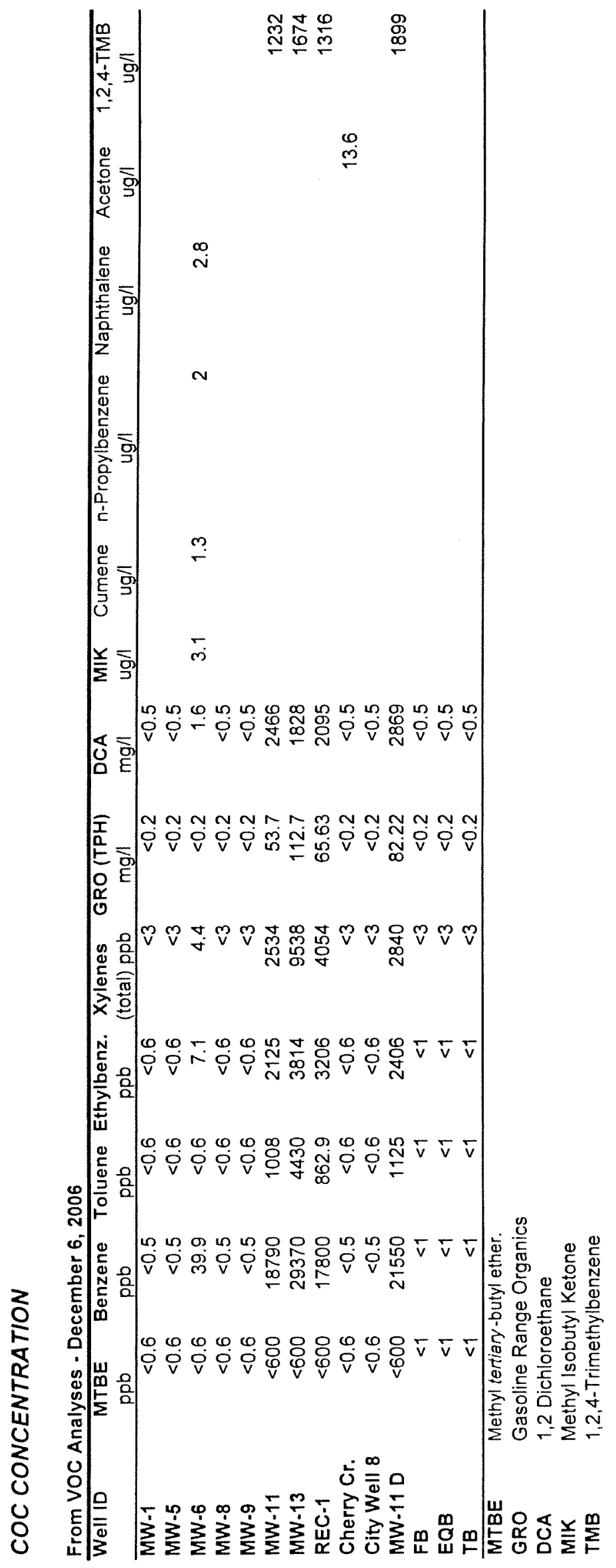




\section{APPENDIX G}

COMPLETE ANALYTICAL DOCUMENTATION 
APPENDIX G-1

GROUNDWATER ANALYSES - COC 
JARDA SOLC

ENERGY \& ENVIRONMENTAL RESEARCH CTR

UNIVERSITY OF NORTH DAKOTA

GRAND FORKS ND 58203

Sample Description: MW-1

W5630
Page: 1 of 2

Report Date: 15 Dec 06

Lab Number: 06-A54130

Work Order \#:82-2656

Account \#: 007033

Sample Matrix: GROUNDWATER

Date Sampled: 6 Dec 06 8:45

Date Received: 8 Dec 06

PO \#: CSI WATFORD

Temp at Receipt: $6.0 \mathrm{C}$

\begin{tabular}{|c|c|c|c|c|c|c|c|c|c|c|}
\hline \multirow[b]{2}{*}{ Chloroethane } & \multicolumn{2}{|c|}{$\begin{array}{l}\text { As Received } \\
\text { Result }\end{array}$} & \multirow{2}{*}{$\begin{array}{l}\text { Method } \\
\text { RL }\end{array}$} & \multicolumn{3}{|c|}{$\begin{array}{l}\text { Method } \\
\text { Reference }\end{array}$} & \multicolumn{3}{|c|}{$\begin{array}{l}\text { Date } \\
\text { Analyzed }\end{array}$} & \multirow{2}{*}{$\begin{array}{l}\text { Analyst } \\
\text { DWR }\end{array}$} \\
\hline & $<0.7$ & $\mathrm{ug} / \mathrm{L}$ & & SW8021 & $\mathrm{MDH}$ & $465 \mathrm{~F}$ & 13 & Dec & 06 & \\
\hline Chlorome thane & $<1$ & $\mathrm{ug} / \mathrm{L}$ & 1 & SW8021 & $\mathrm{MDH}$ & $465 \mathrm{~F}$ & 13 & Dec & 06 & DWR \\
\hline Bromomethane & $<0.7$ & $\mathrm{ug} / \mathrm{L}$ & 0.7 & SW8021 & MDH & $465 \mathrm{~F}$ & 13 & Dec & 06 & DWR \\
\hline Dichlorodifluoromethane & $<0.9$ & $\mathrm{ug} / \mathrm{L}$ & 0.9 & SW8021 & $\mathrm{MDH}$ & $465 \mathrm{~F}$ & 13 & Dec & 06 & DWR \\
\hline Vinyl chloride & $<0.5$ & $\mathrm{ug} / \mathrm{L}$ & 0.5 & SWB02I & MDH & $465 \mathrm{~F}$ & 13 & Dec & 06 & DWR \\
\hline Methylene Chloride & $<0.7$ & $\mathrm{ug} / \mathrm{L}$ & 0.7 & SW8021 & $\mathrm{MDH}$ & $465 \mathrm{~F}$ & 13 & Dec & 06 & DWR \\
\hline Trichlorofluoromethane & $<0.9$ & ug/L & 0.9 & SW8021 & $\mathrm{MDH}$ & $465 \mathrm{~F}$ & 13 & Dec & 06 & DWR \\
\hline 1,1-Dichloroethere & $<0.5$ & $\mathrm{ug} / \mathrm{L}$ & 0.5 & SW8021 & $\mathrm{MDH}$ & $465 \mathrm{~F}$ & 13 & Dec & 06 & DWR \\
\hline 1,1-Dichloroethane & $<0.8$ & $\mathrm{ug} / \mathrm{L}$ & 0.8 & SW802I & $\mathrm{MDH}$ & $465 \mathrm{~F}$ & 13 & Dec & 06 & DWR \\
\hline trans-1,2-Dichloroethene & $<0.5$ & $\mathrm{ug} / \mathrm{L}$ & 0.5 & SW8021 & $\mathrm{MDH}$ & $465 \mathrm{~F}$ & 13 & Dec & 06 & DWR \\
\hline Chloroform & $<0.8$ & $\mathrm{ug} / \mathrm{L}$ & 0.8 & SW8021 & $\mathrm{MDH}$ & $465 \mathrm{~F}$ & 13 & Dec & 06 & DWR \\
\hline 1,2-Dichloroethane & $<0.5$ & $\mathrm{ug} / \mathrm{L}$ & 0.5 & SWB021 & MDH & $465 \mathrm{~F}$ & 13 & Dec & 06 & DWR \\
\hline 1,1,1-Trichloroethane & $<0.9$ & $\mathrm{ug} / \mathrm{L}$ & 0.9 & SW8021 & $\mathrm{MDH}$ & $465 \mathrm{~F}$ & 13 & Dec & 06 & DWR \\
\hline Carbon Tetrachloride & $<0.8$ & $\mathrm{ug} / \mathrm{L}$ & 0.8 & SWB021 & MDH & $465 \mathrm{~F}$ & 13 & Dec & 06 & DWR \\
\hline Bromodichloromethane & $<0.5$ & $\mathrm{ug} / \mathrm{L}$ & 0.5 & SW8021 & $\mathrm{MDH}$ & $465 \mathrm{~F}$ & 13 & Dec & 06 & DWR \\
\hline 1,2-Dichloropropane & $<0.7$ & $\mathrm{ug} / \mathrm{L}$ & 0.7 & SWB021 & MDH & $465 \mathrm{~F}$ & 13 & Dec & 06 & DWR \\
\hline trans - 1,3-Dichloropropene & $<0.5$ & $\mathrm{ug} / \mathrm{L}$ & 0.5 & SW8021 & $\mathrm{MDH}$ & $465 \mathrm{~F}$ & 13 & Dec & 06 & DWR \\
\hline Trichloroethene & $<0.5$ & $\mathrm{ug} / \mathrm{L}$ & 0.5 & SWB021 & $\mathrm{MDH}$ & $465 \mathrm{~F}$ & 13 & Dec & 06 & DWR \\
\hline Chlorodibromome thane & $<0.5$ & $\mathrm{ug} / \mathrm{L}$ & 0.5 & SW8021 & $\mathrm{MDH}$ & $465 \mathrm{~F}$ & 13 & Dec & 06 & DWR \\
\hline 1,1,2-Trichloroethane & $<0.6$ & $\mathrm{ug} / \mathrm{L}$ & 0.6 & SW8021 & $\mathrm{MDH}$ & $465 \mathrm{~F}$ & 13 & Dec & 06 & DWR \\
\hline cis-1,3-Dichloropropene & $<0.5$ & $\mathrm{ug} / \mathrm{L}$ & 0.5 & SW8021 & $\mathrm{MDH}$ & $465 \mathrm{~F}$ & 13 & Dec & 06 & DWR \\
\hline Bromoform & $<1$ & $\mathrm{ug} / \mathrm{L}$ & 1 & SW8021 & $\mathrm{MDH}$ & $465 \mathrm{~F}$ & 13 & Dec & 06 & DWR \\
\hline $1,1,2,2$-Tetrachloroethane & $<0.5$ & $\mathrm{ug} / \mathrm{L}$ & 0.5 & SW8021 & $\mathrm{MDH}$ & $465 \mathrm{~F}$ & 13 & Dec & 06 & DWR \\
\hline Tetrachloroethene & $<0.7$ & $\mathrm{ug} / \mathrm{L}$ & 0.7 & SW8021 & $\mathrm{MDH}$ & $465 \mathrm{~F}$ & 13 & Dec & 06 & DWR \\
\hline Chlorobenzene & $<0.6$ & $\mathrm{ug} / \mathrm{L}$ & 0.6 & SWBO21 & $\mathrm{MDH}$ & $465 \mathrm{~F}$ & 13 & Dec & 06 & DWR \\
\hline Benzene & $<0.5$ & $u g / L$ & 0.5 & SWBO21 & $\mathrm{MDH}$ & $465 \mathrm{~F}$ & 13 & Dec & 06 & DWR \\
\hline Toluene & $<0.6$ & $\mathrm{ug} / \mathrm{L}$ & 0.6 & SWBO21 & $\mathrm{MDH}$ & $465 \mathrm{~F}$ & 13 & Dec & 06 & DWR \\
\hline Ethyl Benzene & $<0.6$ & $\mathrm{ug} / \mathrm{L}$ & 0.6 & SW8021 & MDH & $465 \mathrm{~F}$ & 13 & Dec & 06 & DWR \\
\hline 1,2-Dichlorobenzene & $<0.5$ & $\mathrm{ug} / \mathrm{L}$ & 0.5 & SW8021 & MDH & $465 \mathrm{~F}$ & 13 & Dec & 06 & DWR \\
\hline 1.3-Dichlorobenzene & $<0.5$ & $\mathrm{ug} / \mathrm{L}$ & 0.5 & SW8021 & MDH & $465 \mathrm{~F}$ & 13 & Dec & 06 & DWR \\
\hline 1.4-Dichlorobenzene & $<0.5$ & $\mathrm{ug} / \mathrm{L}$ & 0.5 & $5 W 8021$ & $\mathrm{MDH}$ & $465 \mathrm{~F}$ & 13 & Dec & 06 & DWR \\
\hline cis-1,2-Dichloroethene & $<0.5$ & $\mathrm{ug} / \mathrm{L}$ & 0.5 & SW8021 & $\mathrm{MDH}$ & $465 \mathrm{~F}$ & 13 & Dec & 06 & DWR \\
\hline 1.3-Dichloropropane & $<0.6$ & $\mathrm{ug} / \mathrm{L}$ & 0.6 & SWB021 & MDH & $465 \mathrm{~F}$ & 13 & Dec & 06 & DWR \\
\hline 1.2,3-Trichloropropane & $<0.5$ & $\mathrm{ug} / \mathrm{L}$ & 0.5 & SW8021 & $\mathrm{MDH}$ & $465 \mathrm{~F}$ & 13 & Dec & 06 & DWR \\
\hline Allyl chloride & $<0.7$ & $\mathrm{ug} / \mathrm{L}$ & 0.7 & SWB021 & $\mathrm{MDH}$ & $465 \mathrm{~F}$ & 13 & Dec & 06 & DWR \\
\hline 1.2-Dibromoethane & $<0.5$ & $\mathrm{ug} / \mathrm{L}$ & 0.5 & SW8021 & $\mathrm{MDH}$ & $465 \mathrm{~F}$ & 13 & Dec & 06 & DWR \\
\hline Methyl Ethyl Ketone & $<5$ & $\mathrm{ug} / \mathrm{L}$ & 5 & SWB 021 & $\mathrm{MDH}$ & $465 \mathrm{~F}$ & 13 & Dec & 06 & DWR \\
\hline Methyl Isobutyl Ketone & $<1.4$ & $\mathrm{ug} / \mathrm{L}$ & 1.4 & SWB021 & $\mathrm{MDH}$ & $465 \mathrm{~F}$ & 13 & Dec & 06 & DWR \\
\hline Tetrahydrofuran & $<5$ & $\mathrm{ug} / \mathrm{L}$ & 5 & SWB021 & $\mathrm{MDH}$ & $465 \mathrm{~F}$ & 13 & Dec & 06 & DWR \\
\hline $\mathrm{m}$-xylene and $\mathrm{p}$-xylene & $<0.7$ & $\mathrm{ug} / \mathrm{L}$ & 0.7 & SW8021 & $\mathrm{MDH}$ & $465 \mathrm{~F}$ & 13 & Dec & 06 & DWR \\
\hline o-xylene & $<0.3$ & $\mathrm{ug} / \mathrm{L}$ & 0.3 & SW8021 & MDH & $465 \mathrm{~F}$ & 13 & Dec & 06 & DWR \\
\hline
\end{tabular}

$R L=$ Reporting Limit 


\author{
JARDA SOLC \\ ENERGY \& ENVIRONMENTAL RESEARCH CTR \\ UNIVERSITY OF NORTH DAKOTA \\ GRAND FORKS ND 58203
}

\section{Sample Description: $M W-1$}

W5630

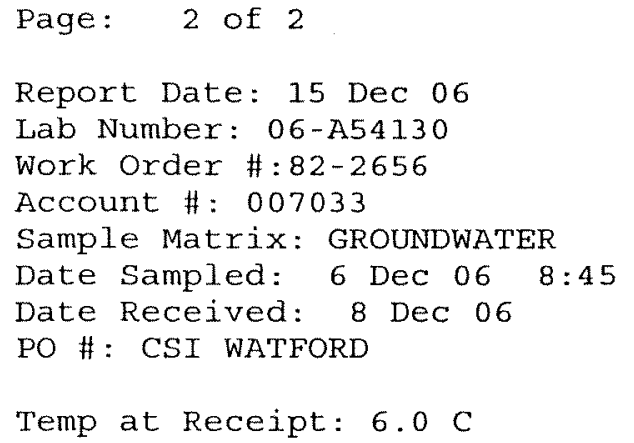

Temp at Receipt: $6.0 \mathrm{C}$

\begin{tabular}{|c|c|c|c|c|c|c|c|c|c|}
\hline \multirow[b]{2}{*}{ Cumene } & \multicolumn{2}{|c|}{$\begin{array}{l}\text { As Received } \\
\text { Result }\end{array}$} & \multirow{2}{*}{$\begin{array}{l}\text { Method } \\
\text { RL } \\
0.7\end{array}$} & \multicolumn{3}{|c|}{$\begin{array}{l}\text { Method } \\
\text { Reference }\end{array}$} & \multicolumn{2}{|c|}{$\begin{array}{l}\text { Date } \\
\text { Analyzed }\end{array}$} & \multirow{2}{*}{$\begin{array}{l}\text { Analyst } \\
\text { DWR }\end{array}$} \\
\hline & $<0.7$ & $\mathrm{ug} / \mathrm{L}$ & & SW802I & $\mathrm{MDH}$ & $465 \mathrm{~F}$ & 13 & Dec 06 & \\
\hline 1,1,1,2-Tetrachloroethane & $<0.5$ & $\mathrm{ug} / \mathrm{L}$ & 0.5 & SW802I & MDH & $465 \mathrm{~F}$ & 13 & Dec 06 & DWR \\
\hline 1.1-Dichloropropene & $<0.5$ & $\mathrm{ug} / \mathrm{L}$ & 0.5 & SWB 021 & MDH & $465 \mathrm{~F}$ & 13 & Dec 06 & DWR \\
\hline Dichlorofluoromethane & $<0.5$ & $\mathrm{ug} / \mathrm{L}$ & 0.5 & SW802I & MDH & $465 \mathrm{~F}$ & 13 & Dec 06 & DWR \\
\hline Trichlorotrifluoroethane & $<0.9$ & $\mathrm{ug} / \mathrm{L}$ & 0.9 & SW8021 & $\mathrm{MDH}$ & $465 \mathrm{~F}$ & 13 & Dec 06 & DWR \\
\hline Ethyl Ether & $<0.8$ & $\mathrm{ug} / \mathrm{L}$ & 0.8 & SW8021 & $\mathrm{MDH}$ & $465 \mathrm{~F}$ & 13 & Dec 06 & DWR \\
\hline Acetone & $<10$ & $\mathrm{ug} / \mathrm{L}$ & 10 & SW8021 & $\mathrm{MDH}$ & $465 \mathrm{~F}$ & 13 & Dec 06 & DWR \\
\hline Dibromomethane & $<0.7$ & $\mathrm{ug} / \mathrm{L}$ & 0.7 & SW8021 & $\mathrm{MDH}$ & $465 \mathrm{~F}$ & 13 & Dec 06 & DWR \\
\hline 2,2-Dichloropropane & $<1$ & $\mathrm{ug} / \mathrm{L}$ & 1 & SW8021 & $\mathrm{MDH}$ & $465 \mathrm{~F}$ & 13 & Dec 06 & DWR \\
\hline Bromochloromethane & $<0.6$ & $\mathrm{ug} / \mathrm{L}$ & 0.6 & SW8021 & MDH & $465 F$ & 13 & Dec 06 & DWR \\
\hline Methyl tert-butyl Ether & $<0.6$ & $\mathrm{ug} / \mathrm{L}$ & 0.6 & SW8 021 & $\mathrm{MDH}$ & $465 \mathrm{~F}$ & 13 & Dec 06 & DWR \\
\hline Styrene & $<0.5$ & $\mathrm{ug} / \mathrm{L}$ & 0.5 & SW8021 & $\mathrm{MDH}$ & $465 \mathrm{~F}$ & 13 & Dec 06 & DWR \\
\hline n-propylbenzene & $<0.8$ & $\mathrm{ug} / \mathrm{L}$ & 0.8 & SW8021 & MDH & $465 \mathrm{~F}$ & 13 & Dec 06 & DWR \\
\hline Bromobenzene & $<0.5$ & $\mathrm{ug} / \mathrm{L}$ & 0.5 & SW8021 & $\mathrm{MDH}$ & $465 \mathrm{~F}$ & 13 & Dec 06 & DWR \\
\hline 2-Chlorotoluene & $<0.8$ & $\mathrm{ug} / \mathrm{L}$ & 0.8 & SW8021 & $\mathrm{MDH}$ & $465 \mathrm{~F}$ & 13 & Dec 06 & DWR \\
\hline 1,3,5-Trimethylbenzene & $<0.9$ & $\mathrm{ug} / \mathrm{L}$ & 0.9 & SW8021 & $\mathrm{MDH}$ & $465 \mathrm{~F}$ & 13 & Dec 06 & DWR \\
\hline 4 -Chlorotoluene & $<0.6$ & $\mathrm{ug} / \mathrm{L}$ & 0.6 & SW8 021 & $\mathrm{MDH}$ & $465 \mathrm{~F}$ & 13 & Dec 06 & DWR \\
\hline t-Butylbenzene & $<0.6$ & $\mathrm{ug} / \mathrm{L}$ & 0.6 & SW8 021 & MDH & $465 \mathrm{~F}$ & 13 & Dec 06 & DWR \\
\hline 1,2,4-Trimethylbenzene & $<0.7$ & $\mathrm{ug} / \mathrm{L}$ & 0.7 & SW8 021 & MDH & $465 \mathrm{~F}$ & 13 & Dec 06 & DWR \\
\hline sec-Butylbenzene & $<0.7$ & $\mathrm{ug} / \mathrm{L}$ & 0.7 & SW8021 & $\mathrm{MDH}$ & $465 \mathrm{~F}$ & 13 & Dec 06 & DWR \\
\hline p-Isopropyltoluene & $<0.7$ & $\mathrm{ug} / \mathrm{L}$ & 0.7 & SW8021 & $\mathrm{MDH}$ & $465 \mathrm{~F}$ & 13 & Dec 06 & DWR \\
\hline n-Butylbenzene & $<0.7$ & $\mathrm{ug} / \mathrm{L}$ & 0.7 & SW8021 & $\mathrm{MDH}$ & $465 \mathrm{~F}$ & 13 & Dec 06 & DWR \\
\hline 1,2-Dibromo-3-chloropropane & $<1$ & $\mathrm{ug} / \mathrm{L}$ & 1 & SW8021. & MDH & $465 \mathrm{~F}$ & 13 & Dec 06 & DWR \\
\hline 1,2,4-Trichlorobenzene & $<0.5$ & $\mathrm{ug} / \mathrm{L}$ & 0.5 & SW8021 & MDH & $465 \mathrm{~F}$ & 13 & Dec 06 & DWR \\
\hline Hexachlorobutadiene & $<0.7$ & $\mathrm{ug} / \mathrm{L}$ & 0.7 & SW8021 & MDH & $465 \mathrm{~F}$ & 13 & Dec 06 & DWR \\
\hline Naphthalene & $<1$ & $\mathrm{ug} / \mathrm{L}$ & 1 & SW8021 & $\mathrm{MDH}$ & $465 \mathrm{~F}$ & 13 & Dec 06 & DWR \\
\hline 1,2,3-Trichlorobenzene & $<0.6$ & $\mathrm{ug} / \mathrm{L}$ & 0.6 & SW8021 & MDH & $465 \mathrm{~F}$ & 13 & Dec 06 & DWR \\
\hline GRO (TPH) & $<0.2$ & $\mathrm{mg} / \mathrm{L}$ & 0.2 & $8015 \mathrm{~B} / \mathrm{O}$ & OAI & & 8 & Dec 06 & $\mathrm{RDC}$ \\
\hline
\end{tabular}

GC VOC Sample pH $<2$

1-CHLORO-4-FLUOROBENZENE (SURROGATE) RECOVERY： $100 \%$

BTEX/GRO Sample $\mathrm{pH}<2$

BTEX SURROGATE RECOVERY: $104 \%$

GRO SURROGATE RECOVERY : $106 \%$ 
JARDA SOLC

ENERGY \& ENVIRONMENTAL RESEARCH CTR

UNIVERSITY OF NORTH DAKOTA

GRAND FORKS ND 58203

Sample Description: MW-5

W5631
Page: 1 of 2

Report Date: 15 Dec 06

Lab Number: 06-A54131

Work Order \#:82-2656

Account \#: 007033

Sample Matrix: GROUNDWATER

Date Sampled: 6 Dec 06 15:15

Date Received: 8 Dec 06

PO \# : CSI WATFORD

Temp at Receipt: $6.0 \mathrm{C}$

\begin{tabular}{|c|c|c|c|c|c|c|c|c|c|c|}
\hline \multirow{2}{*}{$\overline{\text { Chloroethane }}$} & \multicolumn{2}{|c|}{$\begin{array}{l}\text { As Received } \\
\text { Result }\end{array}$} & \multirow{2}{*}{$\begin{array}{l}\text { Method } \\
\text { RL } \\
0.7\end{array}$} & \multicolumn{3}{|c|}{$\begin{array}{l}\text { Method } \\
\text { Reference }\end{array}$} & \multicolumn{3}{|c|}{$\begin{array}{l}\text { Date } \\
\text { Analyzed }\end{array}$} & \multirow{2}{*}{$\frac{\text { Analyst }}{\text { DWR }}$} \\
\hline & $<0.7$ & $\mathrm{ug} / \mathrm{L}$ & & SW8021 & $\mathrm{MDH}$ & $465 \mathrm{~F}$ & 13 & Dec & 06 & \\
\hline Chloromethane & $<1$ & $\mathrm{ug} / \mathrm{L}$ & 1 & SW8021 & $\mathrm{MDH}$ & $465 \mathrm{~F}$ & 13 & Dec & 06 & DWR \\
\hline Bromomethane & $<0.7$ & $\mathrm{ug} / \mathrm{L}$ & 0.7 & SWB 021 & $\mathrm{MDH}$ & $465 \mathrm{~F}$ & 13 & Dec & 06 & DWR \\
\hline Dichlorodifluoromethane & $<0.9$ & $\mathrm{ug} / \mathrm{L}$ & 0.9 & SW8021 & $\mathrm{MDH}$ & $465 \mathrm{~F}$ & 13 & Dec & 06 & DWR \\
\hline Vinyl Chloride & $<0.5$ & $\mathrm{ug} / \mathrm{L}$ & 0.5 & SW8021 & $\mathrm{MDH}$ & $465 \mathrm{~F}$ & 13 & Dec & 06 & DWR \\
\hline Methylene Chloride & $<0.7$ & $\mathrm{ug} / \mathrm{L}$ & 0.7 & SW8021 & $\mathrm{MDH}$ & $465 \mathrm{~F}$ & 13 & Dec & 06 & DWR \\
\hline Trichlorof luorome thane & $<0.9$ & $\mathrm{ug} / \mathrm{L}$ & 0.9 & SW8021 & MDH & $465 \mathrm{~F}$ & 13 & Dec & 06 & DWR \\
\hline 1,1-Dichloroethene & $<0.5$ & $\mathrm{ug} / \mathrm{L}$ & 0.5 & SW8021 & $\mathrm{MDH}$ & $465 \mathrm{~F}$ & 13 & Dec & 06 & DWR \\
\hline 1,1-Dichloroethane & $<0.8$ & $\mathrm{ug} / \mathrm{L}$ & 0.8 & SWB 021 & $\mathrm{MDH}$ & $465 \mathrm{~F}$ & 13 & Dec & 06 & DWR \\
\hline trans-1,2-Dichloroethene & $<0.5$ & $\mathrm{ug} / \mathrm{L}$ & 0.5 & SW8 021 & $\mathrm{MDH}$ & $465 \mathrm{~F}$ & 13 & Dec & 06 & DWR \\
\hline Chloroform & $<0.8$ & $\mathrm{ug} / \mathrm{L}$ & 0.8 & SWB 021 & $\mathrm{MDH}$ & $465 \mathrm{~F}$ & 13 & Dec & 06 & DWR \\
\hline 1.2-Dichloroethane & $<0.5$ & $u g / L$ & 0.5 & SWB 021 & $\mathrm{MDH}$ & $465 \mathrm{~F}$ & 13 & Dec & 06 & DWR \\
\hline 1,1,1-Trichloroe thane & $<0.9$ & $\mathrm{ug} / \mathrm{L}$ & 0.9 & SW8021 & $\mathrm{MDH}$ & $465 \mathrm{~F}$ & 13 & Dec & 06 & DWR \\
\hline Carbon Tetrachloride & $<0.8$ & $\mathrm{ug} / \mathrm{L}$ & 0.8 & SW8021 & $\mathrm{MDH}$ & $465 \mathrm{~F}$ & 13 & Dec & 06 & DWR \\
\hline Bromodichloromethane & $<0.5$ & $\mathrm{ug} / \mathrm{L}$ & 0.5 & SW8021 & $\mathrm{MDH}$ & $465 \mathrm{~F}$ & 13 & Dec & 06 & DWR \\
\hline 1,2-Dichloropropane & $<0.7$ & $\mathrm{ug} / \mathrm{L}$ & 0.7 & SW8021 & MDH & $465 \mathrm{~F}$ & 13 & Dec & 06 & DWR \\
\hline trans-1,3-Dichloropropene & $<0.5$ & $\mathrm{ug} / \mathrm{L}$ & 0.5 & SW802I & MDH & $465 \mathrm{~F}$ & 13 & Dec & 06 & DWR \\
\hline Trichloroethene & $<0.5$ & $\mathrm{ug} / \mathrm{L}$ & 0.5 & SW8021 & MDH & $465 \mathrm{~F}$ & 13 & Dec & 06 & DWR \\
\hline Chlorodibromomethane & $<0.5$ & $\mathrm{ug} / \mathrm{L}$ & 0.5 & SW8021 & $\mathrm{MDH}$ & $465 \mathrm{~F}$ & 13 & Dec & 06 & DWR \\
\hline 1,1,2-Trichloroethane & $<0.6$ & $\mathrm{ug} / \mathrm{L}$ & 0.6 & Sw8021 & $\mathrm{MDH}$ & $465 \mathrm{~F}$ & 13 & Dec & 06 & DWR \\
\hline cis-1,3-Dichloropropene & $<0.5$ & $\mathrm{ug} / \mathrm{L}$ & 0.5 & SWB 021 & $\mathrm{MDH}$ & $465 \mathrm{~F}$ & 13 & Dec & 06 & DWR \\
\hline Bromoform & $<1$ & $\mathrm{ug} / \mathrm{L}$ & 1 & SW8021 & $\mathrm{MDH}$ & $465 \mathrm{~F}$ & 13 & Dec & 06 & DWR \\
\hline 1,1,2,2-Tetrachloroethane & $<0.5$ & $\mathrm{ug} / \mathrm{L}$ & 0.5 & SWB021 & $\mathrm{MDH}$ & $465 \mathrm{~F}$ & 13 & Dec & 06 & DWR \\
\hline Tetrachloroethene & $<0.7$ & $\mathrm{ug} / \mathrm{L}$ & 0.7 & Sw8021 & MDH & $465 \mathrm{~F}$ & 13 & Dec & 06 & DWR \\
\hline Chlorobenzene & $<0.6$ & $\mathrm{ug} / \mathrm{L}$ & 0.6 & SW8021 & $\mathrm{MDH}$ & $465 \mathrm{~F}$ & 13 & Dec & 06 & DWR \\
\hline Benzene & $<0.5$ & $\mathrm{ug} / \mathrm{L}$ & 0.5 & SW8021 & MDH & $465 \mathrm{~F}$ & 13 & Dec & 06 & DWR \\
\hline Toluene & $<0.6$ & $u g / L$ & 0.6 & SW8021 & $\mathrm{MDH}$ & $465 \mathrm{~F}$ & 13 & Dec & 06 & DWR \\
\hline Ethyl Benzene & $<0.6$ & $\mathrm{ug} / \mathrm{L}$ & 0.6 & SWB 021 & $\mathrm{MDH}$ & $465 \mathrm{~F}$ & 13 & Dec & 06 & DWR \\
\hline 1.2-Dichlorobenzene & $<0.5$ & $\mathrm{ug} / \mathrm{L}$ & 0.5 & SW8021 & $\mathrm{MDH}$ & $465 \mathrm{~F}$ & 13 & Dec & 06 & DWR \\
\hline 1.3-Dichlorobenzene & $<0.5$ & $\mathrm{ug} / \mathrm{L}$ & 0.5 & SW8021 & $\mathrm{MDH}$ & $465 \mathrm{~F}$ & 13 & Dec & 06 & DWR \\
\hline 1.4-Dichlorobenzene & $<0.5$ & $\mathrm{ug} / \mathrm{L}$ & 0.5 & SW8021 & MDH & $465 \mathrm{~F}$ & 13 & Dec & 06 & DWR \\
\hline cis-1,2-Dichloroethene & $<0.5$ & $\mathrm{ug} / \mathrm{L}$ & 0.5 & SWB 021 & $\mathrm{MDH}$ & $465 \mathrm{~F}$ & 13 & Dec & 06 & DWR \\
\hline 1,3-Dichloropropane & $<0.6$ & $\mathrm{ug} / \mathrm{L}$ & 0.6 & SW8021 & $\mathrm{MDH}$ & $465 \mathrm{~F}$ & 13 & Dec & 06 & DWR \\
\hline 1,2,3-Trichloropropane & $<0.5$ & $\mathrm{ug} / \mathrm{L}$ & 0.5 & Sw8 021 & $\mathrm{MDH}$ & $465 \mathrm{~F}$ & 13 & Dec & 06 & DWR \\
\hline Allyl Chloride & $<0.7$ & $\mathrm{ug} / \mathrm{L}$ & 0.7 & SW8 021 & MDH & $465 \mathrm{~F}$ & 13 & Dec & 06 & DWR \\
\hline 1.2-Dibromoethane & $<0.5$ & $\mathrm{ug} / \mathrm{L}$ & 0.5 & SW8021 & $\mathrm{MDH}$ & $465 \mathrm{~F}$ & 13 & Dec & 06 & DWR \\
\hline Methyl Ethyl Ketone & $<5$ & $\mathrm{ug} / \mathrm{L}$ & 5 & SW8 021 & $\mathrm{MDH}$ & $465 \mathrm{~F}$ & 13 & Dec & 06 & DWR \\
\hline Methyl Isobutyl Ketone & $<1.4$ & $\mathrm{ug} / \mathrm{L}$ & 1.4 & SW8021 & $\mathrm{MDH}$ & $465 \mathrm{~F}$ & 13 & Dec & 06 & DWR \\
\hline Tetrahydrofuran & $<5$ & $\mathrm{ug} / \mathrm{L}$ & 5 & SW8 021 & $\mathrm{MDH}$ & $465 \mathrm{~F}$ & 13 & Dec & 06 & DWR \\
\hline$m$-xylene and $p$-xylene & $<0.7$ & $\mathrm{ug} / \mathrm{L}$ & 0.7 & SW8 021 & MDH & $465 \mathrm{~F}$ & 13 & Dec & 06 & DWR \\
\hline o-xylene & $<0.3$ & $\mathrm{ug} / \mathrm{L}$ & 0.3 & SW8027 & $\mathrm{MDH}$ & $465 \mathrm{~F}$ & 13 & Dec & 06 & DWR \\
\hline
\end{tabular}

RL = Reporting Limit 


\author{
JARDA SOLC \\ ENERGY \& ENVIRONMENTAL RESEARCH CTR \\ UNIVERSITY OF NORTH DAKOTA \\ GRAND FORKS ND 58203
}

Sample Description: $M W-5$

W5631

\author{
Page: 2 of 2 \\ Report Date: 15 Dec 06 \\ Lab Number: 06-A54131 \\ Work Order \#: 82-2656 \\ Account \#: 007033 \\ Sample Matrix: GROUNDWATER \\ Date Sampled: 6 Dec 06 15:15 \\ Date Received: 8 Dec 06 \\ PO \# : CSI WATFORD \\ Temp at Receipt: $6.0 \mathrm{C}$
}

\begin{tabular}{|c|c|c|c|c|c|c|c|c|c|c|}
\hline \multirow[b]{2}{*}{ Cumene } & \multicolumn{2}{|c|}{$\begin{array}{l}\text { As Received } \\
\text { Result }\end{array}$} & \multirow{2}{*}{$\begin{array}{l}\text { Method } \\
\text { RL } \\
0.7\end{array}$} & \multicolumn{3}{|c|}{$\begin{array}{l}\text { Method } \\
\text { Reference }\end{array}$} & \multicolumn{3}{|c|}{ Date } & \multirow{2}{*}{$\frac{\text { Analyst }}{\text { DWR }}$} \\
\hline & $<0.7$ & $u g / L$ & & SW8021 & $\mathrm{MDH}$ & $465 \mathrm{~F}$ & 13 & Dec & 06 & \\
\hline $1,1,1,2$-Tetrachloroethane & $<0.5$ & $\mathrm{ug} / \mathrm{L}$ & 0.5 & SW8021 & $\mathrm{MDH}$ & $465 \mathrm{~F}$ & 13 & Dec & 06 & DWR \\
\hline 1.1-Dichloropropene & $<0.5$ & $\mathrm{ug} / \mathrm{L}$ & 0.5 & SW8 021 & $\mathrm{MDH}$ & $465 \mathrm{~F}$ & 13 & Dec & 06 & DWR \\
\hline Dichlorofluoromethane & $<0.5$ & $\mathrm{ug} / \mathrm{L}$ & 0.5 & SW8021 & MDH & $465 \mathrm{~F}$ & 13 & Dec & 06 & DWR \\
\hline Trichlorotrifluoroethane & $<0.9$ & $\mathrm{ug} / \mathrm{L}$ & 0.9 & SW8021 & $\mathrm{MDH}$ & $465 F$ & 13 & Dec & 06 & DWR \\
\hline Ethyl Ether & $<0.8$ & $\mathrm{ug} / \mathrm{L}$ & 0.8 & SW8021 & MDH & $465 \mathrm{~F}$ & 13 & Dec & 06 & DWR \\
\hline Acetone & $<10$ & $\mathrm{ug} / \mathrm{L}$ & 10 & SWB 021 & $\mathrm{MDH}$ & $465 \mathrm{~F}$ & 13 & Dec & 06 & DWR \\
\hline Dibromomethane & $<0.7$ & $\mathrm{ug} / \mathrm{L}$ & 0.7 & SW8021. & MDH & $465 \mathrm{~F}$ & 13 & Dec & 06 & DWR \\
\hline 2,2-Dichloropropane & $<1$ & $\mathrm{ug} / \mathrm{L}$ & 1 & SW8021 & $\mathrm{MDH}$ & $465 \mathrm{~F}$ & 13 & Dec & 06 & DWR \\
\hline Bromochloromethane & $<0.6$ & $\mathrm{ug} / \mathrm{L}$ & 0.6 & SW8021 & $\mathrm{MDH}$ & $465 \mathrm{~F}$ & 13 & Dec & 06 & DWR \\
\hline Methyl tert-butyl Ether & $<0.6$ & $\mathrm{ug} / \mathrm{L}$ & 0.6 & SW8021. & $\mathrm{MDH}$ & $465 \mathrm{~F}$ & 13 & Dec & 06 & DWR \\
\hline styrene & $<0.5$ & $\mathrm{ug} / \mathrm{L}$ & 0.5 & SW8021 & $\mathrm{MDH}$ & $465 \mathrm{~F}$ & 13 & Dec & 06 & DWR \\
\hline n-Propylbenzene & $<0.8$ & $\mathrm{ug} / \mathrm{L}$ & 0.8 & SW8021 & $\mathrm{MDH}$ & $465 \mathrm{~F}$ & 13 & Dec & 06 & DWR \\
\hline Bromobenzene & $<0.5$ & $\mathrm{ug} / \mathrm{I}$ & 0.5 & SW8021 & MDH & $465 \mathrm{~F}$ & 13 & Dec & 06 & DWR \\
\hline 2-Chlorotoluene & $<0.8$ & $\mathrm{ug} / \mathrm{L}$ & 0.8 & SW8021 & $\mathrm{MDH}$ & $465 \mathrm{~F}$ & 13 & Dec & 06 & DWR \\
\hline $1,3,5$-Trimethylbenzene & $<0.9$ & $\mathrm{ug} / \mathrm{L}$ & 0.9 & SW8021 & $\mathrm{MDH}$ & $465 \mathrm{~F}$ & 13 & Dec & 06 & DWR \\
\hline 4-Chlorotoluene & $<0.6$ & $\mathrm{ug} / \mathrm{L}$ & 0.6 & SWBO2I & MDH & $465 F$ & 13 & Dec & 06 & DWR \\
\hline t-Butylbenzene & $<0.6$ & $\mathrm{ug} / \mathrm{L}$ & 0.6 & SW8021 & $\mathrm{MDH}$ & $465 F$ & 13 & Dec & 06 & DWR \\
\hline $1,2,4$-Trimethylbenzene & $<0.7$ & $\mathrm{ug} / \mathrm{L}$ & 0.7 & SW8021 & $\mathrm{MDH}$ & $465 \mathrm{~F}$ & 13 & Dec & 06 & DWR \\
\hline sec-Butylbenzene & $<0.7$ & $\mathrm{ug} / \mathrm{L}$ & 0.7 & SW8021 & $\mathrm{MDH}$ & $465 \mathrm{~F}$ & 13 & Dec & 06 & DWR \\
\hline$p$-Isopropyltoluene & $<0.7$ & $\mathrm{ug} / \mathrm{L}$ & 0.7 & SW8021 & MDH & $465 \mathrm{~F}$ & 13 & Dec & 06 & DWR \\
\hline n-Butylbenzene & $<0.7$ & $\mathrm{ug} / \mathrm{L}$ & 0.7 & SW8021 & MDH & $465 \mathrm{~F}$ & 13 & Dec & 06 & DWR \\
\hline 1,2-Dibromo-3-chloropropane & $<1$ & $u g / L$ & 1 & SW8021 & MDH & $465 \mathrm{~F}$ & 13 & Dec & 06 & DWR \\
\hline 1.2.4-Trichlorobenzene & $<0.5$ & $\mathrm{ug} / \mathrm{L}$ & 0.5 & $5 W 8021$ & $\mathrm{MDH}$ & $465 \mathrm{~F}$ & 13 & Dec & 06 & DWR \\
\hline Hexachlorobutadiene & $<0.7$ & $\mathrm{ug} / \mathrm{L}$ & 0.7 & SWBO21 & $\mathrm{MDH}$ & $465 \mathrm{~F}$ & 13 & Dec & 06 & DWR \\
\hline Naphthalene & $<1$ & $\mathrm{ug} / \mathrm{L}$ & 1 & SWBO21 & $\mathrm{MDH}$ & $465 \mathrm{~F}$ & 13 & Dec & 06 & DWR \\
\hline 1,2,3-Trichlorobenzene & $<0.6$ & $\mathrm{ug} / \mathrm{L}$ & 0.6 & SW8021 & MDH & $465 \mathrm{~F}$ & 13 & DeC & 06 & DWR \\
\hline GRO (TPH) & $<0.2$ & $\mathrm{mg} / \mathrm{L}$ & 0.2 & $8015 B / C$ & $D A 1$ & & B & Dec & 06 & RDC \\
\hline
\end{tabular}

GC VOC Sample $\mathrm{pH}<2$

1-CHLORO-4-FLUOROBENZENE (SURROGATE) RECOVERY: $101 \%$

BTEX/GRO Sample pH $<2$

BTEX SURROGATE RECOVERY: $104 \%$

GRO SURROGATE RECOVERY : $97 \%$

$R L=$ Reporting Limit 


\author{
JARDA SOLC \\ ENERGY \& ENVIRONMENTAL RESEARCH CTR \\ UNIVERSITY OF NORTH DAKOTA \\ GRAND FORKS ND 58203
}

Sample Description: $M W-6$

W5 632

\author{
Page: 1 of 2 \\ Report Date: 15 Dec 06 \\ Lab Number: 06-A54132 \\ work Order \#:82-2656 \\ Account \#: 007033 \\ Sample Matrix: GROUNDWATER \\ Date Sampled: 6 Dec 06 13:55 \\ Date Received: 8 Dec 06 \\ PO \#: CSI WATFORD \\ Temp at Receipt: $6.0 \mathrm{C}$
}

\begin{tabular}{|c|c|c|c|c|c|c|c|c|c|c|}
\hline \multirow[b]{2}{*}{ Chloroethane } & \multicolumn{2}{|c|}{$\begin{array}{l}\text { As Received } \\
\text { Result }\end{array}$} & \multirow{2}{*}{$\begin{array}{l}\text { Method } \\
\text { RL } \\
0.7\end{array}$} & \multicolumn{3}{|c|}{$\begin{array}{l}\text { Method } \\
\text { Reference }\end{array}$} & \multicolumn{3}{|c|}{$\begin{array}{l}\text { Date } \\
\text { Analyzed }\end{array}$} & \multirow{2}{*}{$\begin{array}{l}\text { Analyst } \\
\text { DWR }\end{array}$} \\
\hline & $<0.7$ & $\mathrm{ug} / \mathrm{L}$ & & SW8021 & $\mathrm{MDH}$ & $465 \mathrm{~F}$ & 13 & Dec & 06 & \\
\hline Chloromethane & $<1$ & $\mathrm{ug} / \mathrm{L}$ & 1 & SW8021 & $\mathrm{MDH}$ & $465 \mathrm{~F}$ & 13 & Dec & 06 & DWR \\
\hline Bromomethane & $<0.7$ & $\mathrm{ug} / \mathrm{L}$ & 0.7 & SW8021 & $\mathrm{MDH}$ & $465 \mathrm{~F}$ & 13 & Dec & 06 & DWR \\
\hline Dichlorodifluoromethane & $<0.9$ & $\mathrm{ug} / \mathrm{L}$ & 0.9 & SW8021 & MDH & $465 \mathrm{~F}$ & 13 & Dec & 06 & DWR \\
\hline Vinyl chloride & $<0.5$ & $\mathrm{ug} / \mathrm{I}$ & 0.5 & SW8021 & MDH & $465 \mathrm{~F}$ & 13 & DeC & 06 & DWR \\
\hline Methylene Chloride & $<0.7$ & $\mathrm{ug} / \mathrm{L}$ & 0.7 & SW8021 & $\mathrm{MDH}$ & $465 \mathrm{~F}$ & 13 & Dec & 06 & DWR \\
\hline Trichlorof luorome thane & $<0.9$ & $\mathrm{ug} / \mathrm{L}$ & 0.9 & SW8021 & $\mathrm{MDH}$ & $465 \mathrm{~F}$ & 13 & Dec & 06 & DWR \\
\hline 1,1-Dichloroethene & $<0.5$ & $\mathrm{ug} / \mathrm{L}$ & 0.5 & SW8021 & MDH & $465 \mathrm{~F}$ & 13 & Dec & 06 & DWR \\
\hline 1,1-Dichloroethane & $<0.8$ & $\mathrm{ug} / \mathrm{L}$ & 0.8 & SW8021 & MDH & $465 \mathrm{~F}$ & 13 & Dec & 06 & DWR \\
\hline $\operatorname{trans}-1,2$-Dichloroethene & $<0.5$ & $\mathrm{ug} / \mathrm{L}$ & 0.5 & 5W8021 & MDH & $465 \mathrm{~F}$ & 13 & Dec & 06 & DWR \\
\hline Chloroform & $<0.8$ & $\mathrm{ug} / \mathrm{L}$ & 0.8 & SW8021 & MDH & $465 \mathrm{~F}$ & 13 & Dec & 06 & DWR \\
\hline 1,2-Dichloroethane & 1.6 & $\mathrm{ug} / \mathrm{L}$ & 0.5 & SW8021 & MDH & $465 F$ & 13 & Dec & 06 & DWR \\
\hline $1,1,1$-Trichloroethane & $<0.9$ & $\mathrm{ug} / \mathrm{L}$ & 0.9 & SW8021 & MDH & $465 \mathrm{~F}$ & 13 & Dec & 06 & DWR \\
\hline Carbon Tetrachloride & $<0.8$ & $\mathrm{ug} / \mathrm{L}$ & 0.8 & SW8021 & MDH & $465 \mathrm{~F}$ & 13 & Dec & 06 & DWR \\
\hline Bromodichloromethane & $<0.5$ & $\mathrm{ug} / \mathrm{I}$ & 0.5 & SW8021 & MDH & $465 \mathrm{~F}$ & 13 & Dec & 06 & DWR \\
\hline 1,2-Dichloropropane & $<0.7$ & $\mathrm{ug} / \mathrm{L}$ & 0.7 & SW8021 & MDH & $465 F$ & 13 & Dec & 06 & DWR \\
\hline trans-1,3-Dichloropropene & $=0.5$ & $\mathrm{ug} / \mathrm{L}$ & 0.5 & SW8021 & MDH & $465 \mathrm{~F}$ & 13 & Dec & 06 & DWR \\
\hline Trichloroethene & $<0.5$ & $\mathrm{ug} / \mathrm{L}$ & 0.5 & Sw8021 & MDH & $465 \mathrm{~F}$ & 13 & Dec & 06 & DWR \\
\hline Chlorodibromomethane & $<0.5$ & $\mathrm{ug} / \mathrm{L}$ & 0.5 & Sw8021 & MDH & $465 \mathrm{~F}$ & 13 & Dec & 06 & DWR \\
\hline 1,1,2-Trichloroethane & $<0.6$ & $\mathrm{ug} / \mathrm{I}$ & 0.6 & SWB021 & MDH & $465 \mathrm{~F}$ & 13 & Dec & 06 & DWR \\
\hline cis-1,3-Dichloropropene & $<0.5$ & $\mathrm{ug} / \mathrm{L}$ & 0.5 & SW8021 & MDH & $465 \mathrm{~F}$ & 13 & Dec & 06 & DWR \\
\hline Bromoform & $<1$ & $\mathrm{ug} / \mathrm{L}$ & 1 & SW8021 & MDH & $465 \mathrm{~F}$ & 13 & Dec & 06 & DWR \\
\hline $1,1,2,2$-Tetrachloroethane & $<0.5$ & $\mathrm{ug} / \mathrm{L}$ & 0.5 & SW8021 & MDH & $465 \mathrm{~F}$ & 13 & Dec & 06 & DWR \\
\hline Tetrachloroethene & $<0.7$ & $\mathrm{ug} / \mathrm{L}$ & 0.7 & SWB021 & MDH & $465 \mathrm{~F}$ & 13 & Dec & 06 & DWR \\
\hline Chlorobenzene & $<0.6$ & $\mathrm{ug} / \mathrm{L}$ & 0.6 & SWB021 & $\mathrm{MDH}$ & $465 \mathrm{~F}$ & 13 & Dec & 06 & DWR \\
\hline Benzene & 39.9 & $\mathrm{ug} / \mathrm{L}$ & 0.5 & SW8021 & MDH & $465 \mathrm{~F}$ & 13 & Dec & 06 & DWR \\
\hline Toluene & $<0.6$ & $\mathrm{ug} / \mathrm{I}$ & 0.6 & SW8021 & $\mathrm{MDH}$ & $465 \mathrm{~F}$ & 13 & Dec & 06 & DWR \\
\hline Ethyl Benzene & 7.1 & $\mathrm{ug} / \mathrm{L}$ & 0.6 & SW8021 & MDH & $465 \mathrm{~F}$ & 13 & Dec & 06 & DWR \\
\hline 1,2-Dichlorobenzene & $<0.5$ & $\mathrm{ug} / \mathrm{L}$ & 0.5 & SW8021 & $\mathrm{MDH}$ & $465 \mathrm{~F}$ & 13 & Dec & 06 & DWR \\
\hline 1,3-Dichlorobenzene & $<0.5$ & $u g / L$ & 0.5 & SWB 021 & $\mathrm{MDH}$ & $465 \mathrm{~F}$ & 13 & Dec & 06 & DWR \\
\hline 1,4-Dichlorobenzene & $<0.5$ & $\mathrm{ug} / \mathrm{L}$ & 0.5 & SWB021 & $\mathrm{MDH}$ & $465 \mathrm{~F}$ & 13 & Dec & 06 & DWR \\
\hline cis-1,2-Dichloroethene & $<0.5$ & $\mathrm{ug} / \mathrm{L}$ & 0.5 & SW8 021 & MDH & $465 \mathrm{~F}$ & 13 & Dec & 06 & DWR \\
\hline 1,3-Dichloropropane & $=0.6$ & $\mathrm{ug} / \mathrm{L}$ & 0.6 & SW8021 & $\mathrm{MDH}$ & $465 \mathrm{~F}$ & 13 & Dec & 06 & DWR \\
\hline 1,2,3-Trichloropropane & $<0.5$ & $\mathrm{ug} / \mathrm{L}$ & 0.5 & SW8021 & MDH & $465 \mathrm{~F}$ & 13 & Dec & 06 & DWR \\
\hline Allyl Chloride & $<0.7$ & $\mathrm{ug} / \mathrm{L}$ & 0.7 & SW8021 & $\mathrm{MDH}$ & $465 \mathrm{~F}$ & 1.3 & Dec & 06 & DWR \\
\hline 1.2-Dibromoethane & $<0.5$ & $\mathrm{ug} / \mathrm{L}$ & 0.5 & SW8021 & MDH & $465 \mathrm{~F}$ & 13 & Dec & 06 & DWR \\
\hline Methyl Ethyl Ketone & $<5$ & $\mathrm{ug} / \mathrm{L}$ & 5 & Sw8021 & MDH & $465 \mathrm{~F}$ & 13 & Dec & 06 & DWR \\
\hline Methyl Isobutyl Ketone & 3.1 & $\mathrm{ug} / \mathrm{L}$ & 1.4 & SW8021 & $\mathrm{MDH}$ & $465 \mathrm{~F}$ & 13 & Dec & 06 & DWR \\
\hline Tetrahydrofuran & $<5$ & $\mathrm{ug} / \mathrm{L}$ & 5 & SW8 021 & $\mathrm{MDH}$ & $465 \mathrm{~F}$ & 13 & Dec & 06 & DWR \\
\hline m-Xylene and p-Xylene & 4.4 & $\mathrm{ug} / \mathrm{L}$ & 0.7 & SW8021 & $\mathrm{MDH}$ & $465 \mathrm{~F}$ & 13 & Dec & 06 & DWR \\
\hline & & & 0.3 & SW8021 & $\mathrm{MDH}$ & $465 \mathrm{~F}$ & 13 & Dec & 06 & DWR \\
\hline
\end{tabular}

RL = Reporting Limit 
JARDA SOLC

ENERGY \& ENVIRONMENTAL RESEARCH CTR

UNIVERSITY OF NORTH DAKOTA

GRAND FORKS ND 58203

\section{Sample Description: MW-6}

W5632

\begin{tabular}{|c|c|c|c|c|c|c|c|c|c|c|}
\hline \multirow[b]{2}{*}{ Cumene } & \multicolumn{2}{|c|}{$\begin{array}{l}\text { As Received } \\
\text { Result }\end{array}$} & \multirow{2}{*}{$\begin{array}{l}\text { Method } \\
\text { RL. } \\
0.7\end{array}$} & \multicolumn{3}{|c|}{$\begin{array}{l}\text { Method } \\
\text { Reference }\end{array}$} & \multicolumn{3}{|c|}{$\begin{array}{l}\text { Date } \\
\text { Analyzed }\end{array}$} & \multirow{2}{*}{$\begin{array}{l}\text { Analyst } \\
\text { DWR }\end{array}$} \\
\hline & 1.3 & $\mathrm{ug} / \mathrm{L}$ & & SWB021 & $\mathrm{MDH}$ & $465 \mathrm{~F}$ & 13 & $\operatorname{Dec} 0$ & 06 & \\
\hline $1,1,1,2$-Tetrachloroethane & $<0.5$ & $\mathrm{ug} / \mathrm{L}$ & 0.5 & SW8021 & $\mathrm{MDH}$ & $465 \mathrm{~F}$ & 13 & Dec 0 & 06 & DWR \\
\hline 1.1-Dichloropropene & $<0.5$ & $\mathrm{ug} / \mathrm{L}$ & 0.5 & SW8021 & $\mathrm{MDH}$ & $465 \mathrm{~F}$ & 13 & Dec 0 & 06 & DWR \\
\hline Dichlorofluoromethane & $<0.5$ & $\mathrm{ug} / \mathrm{L}$ & 0.5 & SW8021 & $\mathrm{MDH}$ & $465 \mathrm{~F}$ & 13 & Dec 0 & 06 & DWR \\
\hline Trichlorotrifluoroethane & $<0.9$ & $\mathrm{ug} / \mathrm{L}$ & 0.9 & SWB021 & $\mathrm{MDH}$ & $465 \mathrm{~F}$ & 13 & Dec 0 & 06 & DWR \\
\hline Ethyl Ether & $<0.8$ & $\mathrm{ug} / \mathrm{L}$ & 0.8 & SW8021 & MDH & $465 \mathrm{~F}$ & 13 & Dec 0 & 06 & DWR \\
\hline Acetone & $<10$ & $\mathrm{ug} / \mathrm{L}$ & 10 & SW8021 & MDH & $465 \mathrm{~F}$ & 13 & Dec 0 & 06 & DWR \\
\hline Dibromomethane & $<0.7$ & $\mathrm{ug} / \mathrm{L}$ & 0.7 & SW8021 & MDH & $465 \mathrm{~F}$ & 13 & Dec $c$ & 06 & DWR \\
\hline 2.2-Dichloropropane & $<1$ & $\mathrm{ug} / \mathrm{L}$ & 1 & SW8021 & MDH & $465 \mathrm{~F}$ & 13 & Dec 0 & 06 & DWR \\
\hline Bromochloromethane & $<0.6$ & $\mathrm{ug} / \mathrm{L}$ & 0.6 & SW8021 & MDH & $465 \mathrm{~F}$ & 13 & Dec 0 & 06 & DWR \\
\hline Methyl tert-butyl Ether & $<0.6$ & $\mathrm{ug} / \mathrm{L}$ & 0.6 & SW8021 & MDH & $465 \mathrm{~F}$ & 13 & Dec 0 & 06 & DWR \\
\hline styrene & $<0.5$ & $\mathrm{ug} / \mathrm{L}$ & 0.5 & SW8021 & $\mathrm{MDH}$ & $465 \mathrm{~F}$ & 13 & Dec 0 & 06 & DWR \\
\hline n-Propylbenzene & 2.0 & $\mathrm{ug} / \mathrm{L}$ & 0.8 & SW8021 & $\mathrm{MDH}$ & $465 F$ & 13 & Dec 0 & 06 & DWR \\
\hline Bromobenzene & $<0.5$ & $\mathrm{ug} / \mathrm{L}$ & 0.5 & Sw8 021 & MDH & $465 \mathrm{~F}$ & 13 & Dec 0 & 06 & DWR \\
\hline 2-Chlorotoluene & $<0.8$ & $\mathrm{ug} / \mathrm{L}$ & 0.8 & SWB 021 & $\mathrm{MDH}$ & $465 \mathrm{~F}$ & 13 & Dec 0 & 06 & DWR \\
\hline 1,3,5-Trimethylbenzene & $<0.9$ & $\mathrm{ug} / \mathrm{L}$ & 0.9 & SW8021 & $\mathrm{MDH}$ & $465 \mathrm{~F}$ & 13 & Dec $c$ & 06 & DWR \\
\hline 4-chlorotoluene & $<0.6$ & $\mathrm{ug} / \mathrm{L}$ & 0.6 & SW8 021 & MDH & $465 \mathrm{~F}$ & 13 & Dec $c$ & 06 & DWR \\
\hline t-Butylbenzene & $<0.6$ & $\mathrm{ug} / \mathrm{L}$ & 0.6 & SW8021 & $\mathrm{MDH}$ & $465 \mathrm{~F}$ & 13 & Dec $c$ & 06 & DWR \\
\hline 1,2,4-Trimethylbenzene & 0.8 & $\mathrm{ug} / \mathrm{L}$ & 0.7 & SW8021 & $\mathrm{MDH}$ & $465 \mathrm{~F}$ & 13 & Dec $c$ & 06 & DWR \\
\hline sec-Butylbenzene & $<0.7$ & $\mathrm{ug} / \mathrm{L}$ & 0.7 & SW8021 & $\mathrm{MDH}$ & $465 \mathrm{~F}$ & 13 & Dec $c$ & 06 & DWR \\
\hline p-Isopropyl tol uene & $<0.7$ & $\mathrm{ug} / \mathrm{L}$ & 0.7 & SW8021 & $\mathrm{MDH}$ & $465 \mathrm{~F}$ & 13 & Dec & 06 & DWR \\
\hline n-Butylbenzene & $<0.7$ & $\mathrm{ug} / \mathrm{L}$ & 0.7 & SW8021 & $\mathrm{MDH}$ & $465 \mathrm{~F}$ & 13 & Dec & 06 & DWR \\
\hline 1.2-Dibromo-3-chloropropane & $<1$ & $\mathrm{ug} / \mathrm{L}$ & 1 & SW8021 & $\mathrm{MDH}$ & $465 \mathrm{~F}$ & 13 & Dec & 06 & DWR \\
\hline 1,2,4-Trichlorobenzene & $<0.5$ & $\mathrm{ug} / \mathrm{L}$ & 0.5 & SW8021 & $\mathrm{MDH}$ & $465 \mathrm{~F}$ & 13 & Dec & 06 & DWR \\
\hline Hexachlorobutadiene & $<0.7$ & $\mathrm{ug} / \mathrm{L}$ & 0.7 & SW8021 & $\mathrm{MDH}$ & $465 \mathrm{~F}$ & 13 & Dec & 06 & DWR \\
\hline Naphthalene & 2.8 & $\mathrm{ug} / \mathrm{L}$ & 1.0 & SW8021 & $\mathrm{MDH}$ & $465 \mathrm{~F}$ & 13 & Dec & 06 & DWR \\
\hline 1,2,3-Trichlorobenzene & $<0.6$ & $\mathrm{ug} / \mathrm{L}$ & 0.6 & SW8021 & $\mathrm{MDH}$ & $465 \mathrm{~F}$ & 13 & Dec & 06 & DWR \\
\hline GRO (TPH) & $<0.2$ & $\mathrm{mg} / \mathrm{L}$ & 0.2 & $8015 \mathrm{~B} / \mathrm{C}$ & OA1 & & 8 & Dec & 06 & $\mathrm{RDC}$ \\
\hline
\end{tabular}

GC VOC sample $\mathrm{pH}<2$

1-CHLORO-4-FLUOROBENZENE (SURROGATE) RECOVERY: $100 \%$

BTEX/GRO Sample pH $<2$

BTEX SURROGATE RECOVERY: $109 \%$

GRO SURROGATE RECOVERY: $109 \%$

Work Order \#:82-2656
Page: 2 of 2

Report Date: 15 Dec 06

Lab Number: 06-A54132

Account \#: 007033

Sample Matrix: GROUNDWATER

Date Sampled: 6 Dec 06 13:55

Date Received: 8 Dec 06

PO \# : CSI WATFORD

Temp at Receipt: $6.0 \mathrm{C}$ 
JARDA SOLC

ENERGY \& ENVIRONMENTAL RESEARCH CTR

UNIVERSITY OF NORTH DAKOTA

GRAND FORKS ND 58203

Sample Description: MW-8

W5633

As Received

Result

Chloroethane

Chloromethane

Bromomethane

Dichlorodifluoromethane

vinyl Chloride

Methylene chloride

Trichlor of luorome thane

1.1-Dichloroethene

1,1-Dichloroethane

trans-1,2-Dichloroethene

Chloroform

1,2-Dichloroethane

1,1,1-Trichloroet hane

Carbon Tetrachloride

Bromodichlorome thane

1,2-Dichloropropane

trans-1, 3-Dichloropropene

Trichloroethene

Chlorodibromome thane

1,1,2-Trichloroethane

cis-1,3-Dichloropropene

Bromoform

1, 1, 2,2-Tetrachloroethane

Tetrachloroethene

Chlorobenzene

Benzene

Toluene

Ethyl Benzene

1,2-Dichlorobenzene

1,3-Dichlorobenzene

1. 4-Dichlorobenzene

Cis-1,2-Dichloroethene

1,3-Dichloropropane

1,2,3-Trichloropropane

Allyl Chloride

1,2-Dibromoethane

Methyl Ethyl Ketone

Methyl Isobutyl Ketone

Tetrahydrofuran

$m-x y l e n e$ and $p-x y l e n e$

o-xylene

$<0.7 \quad \mathrm{ug} /$

$<1 \quad \mathrm{ug} / \mathrm{L}$

$<0.7$

$<0.9$

$<0.5$

$<0.7$

$<0.9$

$<0.5$

$<0.8$

$<0.5$

$<0.8$

$<0.5$

$<0.9$

$<0.8$

$<0.5$

$<0.7$

$<0.5$

$<0.5$

$<0.5$

$<0.6$

$<0.5$

$<1$

$<0.5$

$<0.7$

$<0.6$

$<0.5$

$<0.6$

$<0.6$

$<0.5$

$<0.5$

$<0.5$

$<0.5$

$<0.6$

$<0.5$

$<0.7$

$<0.5$

$<5$

$<1.4$

$<5$

$<0.7$

$<0.3$ $\mathrm{ug} / \mathrm{L}$

$\mathrm{ug} / \mathrm{L}$

ug/L

$\mathrm{ug} / \mathrm{L}$

$\mathrm{ug} / \mathrm{L}$

$u g / L$

$\mathrm{ug} / \mathrm{L}$

$\mathrm{ug} / \mathrm{L}$

$\mathrm{ug} / \mathrm{L}$

$\mathrm{ug} / \mathrm{L}$,

$u g / L$

$\mathrm{ug} / \mathrm{L}$,

$\mathrm{ug} / \mathrm{L}$

ug/L

$\mathrm{ug} / \mathrm{L}$

$\mathrm{ug} / \mathrm{L}$

$\mathrm{ug} / \mathrm{L}$

$\mathrm{ug} / \mathrm{L}$

$\mathrm{ug} / \mathrm{L}$

$\mathrm{ug} / \mathrm{L}$

$\mathrm{ug} / \mathrm{L}$

$\mathrm{ug} / \mathrm{L}$

$u g / L$

ug $/ 1$.

$\mathrm{ug} / \mathrm{L}$

ug/L

$\mathrm{ug} / \mathrm{L}$

$\mathrm{ug} / \mathrm{L}$

$\mathrm{ug} / \mathrm{L}$

$\mathrm{ug} / \mathrm{L}$

$\mathrm{ug} / \mathrm{L}$.

$\mathrm{ug} / \mathrm{L}$

$\mathrm{ug} / \mathrm{L}$

$\mathrm{ug} / \mathrm{L}$

$\mathrm{ug} / \mathrm{L}$

$\mathrm{ug} / \mathrm{L}$

$\mathrm{ug} / \mathrm{I}$

$\mathrm{ug} / \mathrm{L}$

$\mathrm{ug} / \mathrm{L}$
Page: 1 of 2

Report Date: 15 Dec 06

Lab Number: 06-A54133

Work Order \#:82-2656

Account \#: 007033

Sample Matrix: GROUNDWATER

Date Sampled: 6 Dec 06 13:30

Date Received: 8 Dec 06

PO \#: CSI WATFORD

Temp at Receipt: $6.0 \mathrm{C}$
Method Date

Reference Analyzed

13 Dec 06

13 DeC 06

13 DeC 06

13 Dec 06

13 Dec 06

13 Dec 06

13 Dec 06

13 DeC $O 6$

13 Dec 06

13 Dec 06

13 Dec 06

13 DeC 06

13 DeC 06

13 Dec 06

13 DeC 06

13 Dec 06

13 Dec 06

13 Dec 06

13 Dec 06

13 Dec 06

13 Dec 06

13 DeC 06

13 Dec 06

13 Dec 06

13 Dec 06

13 Dec 06

13 Dec 06

13 DeC 06

13 Dec 06

13 Dec 06

13 Dec 06

13 Dec 06

13 Dec 06

13 Dec 06

13 Dec 06

13 Dec 06

13 Dec 06

13 Dec 06

13 Dec 06

13 DeC 06

13 Dec 06
Analyst

DWR

DWR

DWR

DWR

DWR

DWR

DWR

DWR

DWR

DWR

DWR

DWR

DWR

DWR

DWR

DWR

DWR

DWR

DWR

DWR

DWR

DWR

DWR

DWR

DWR

DWR

DWR

DWR

DWR

DWR

DWR

DWR

DWR

DWR

DWR

DWR

DWR

DWR

DWR

DWR

DWR

$R L=$ Reporting Limit 
JARDA SOLC

ENERGY \& ENVIRONMENTAL RESEARCH CTR

UNIVERSITY OF NORTH DAKOTA

GRAND FORKS ND 58203

\section{Sample Description: $M W-8$}

W5633

\begin{tabular}{|c|c|c|c|c|c|c|c|c|c|c|}
\hline \multirow[b]{2}{*}{ Cumene } & \multicolumn{2}{|c|}{$\begin{array}{l}\text { As Received } \\
\text { Result }\end{array}$} & \multirow{2}{*}{$\begin{array}{l}\text { Method } \\
\text { RL. } \\
0.7\end{array}$} & \multicolumn{3}{|c|}{$\begin{array}{l}\text { Method } \\
\text { Reference }\end{array}$} & \multicolumn{3}{|c|}{$\begin{array}{l}\text { Date } \\
\text { Analyzed }\end{array}$} & \multirow{2}{*}{$\frac{\text { Analyst }}{\text { DWR }}$} \\
\hline & $<0.7$ & $\mathrm{ug} / \mathrm{L}$ & & SW8021 & MDH & $465 \mathrm{~F}$ & 13 & Dec 0 & 06 & \\
\hline $1,1,1,2$-Tetrachloroethane & $<0.5$ & $\mathrm{ug} / \mathrm{L}$ & 0.5 & SW8021 & $\mathrm{MDH}$ & $465 \mathrm{~F}$ & 13 & Dec 0 & 06 & DWR \\
\hline 1.1-Dichloropropene & $<0.5$ & $\mathrm{ug} / \mathrm{L}$ & 0.5 & SW8021 & MDH 4 & $465 \mathrm{~F}$ & 13 & Dec 0 & 06 & DWR \\
\hline Dichlorofluoromethane & $<0.5$ & $\mathrm{ug} / \mathrm{L}$ & 0.5 & SW802I & $\mathrm{MDH}$ & $465 \mathrm{~F}$ & 13 & Dec 0 & 06 & DWR \\
\hline Trichlorotrifluoroethane & $<0.9$ & $\mathrm{ug} / \mathrm{L}$ & 0.9 & Sw8021. & MDH & $465 \mathrm{~F}$ & 13 & Dec 0 & 06 & DWR \\
\hline Ethyl Ether & $<0.8$ & $\mathrm{ug} / \mathrm{L}$ & 0.8 & SWB 021 & $\mathrm{MDH}$ & $465 \mathrm{~F}$ & 13 & Dec 0 & 06 & DWR \\
\hline Acetone & $<10$ & $\mathrm{ug} / \mathrm{L}$ & 10 & SW8021 & $\mathrm{MDH}$ & $465 \mathrm{~F}$ & 13 & Dec 0 & 06 & DWR \\
\hline Dibromomethane & $<0.7$ & $\mathrm{ug} / \mathrm{L}$ & 0.7 & SW8021 & MDH & $465 \mathrm{~F}$ & 13 & Dec 0 & 06 & DWR \\
\hline 2,2-Dichloropropane & $<1$ & $\mathrm{ug} / \mathrm{L}$ & 1 & SW8021 & $\mathrm{MDH}$ & $465 \mathrm{~F}$ & 13 & Dec 0 & 06 & DWR \\
\hline Bromochlorome thane & $<0.6$ & $\mathrm{ug} / \mathrm{L}$ & 0.6 & SW8021 & $\mathrm{MDH}$ & $465 \mathrm{~F}$ & 13 & Dec 0 & 06 & DWR \\
\hline Methyl tert-butyl Ether & $<0.6$ & $\mathrm{ug} / \mathrm{L}$ & 0.6 & SW8021 & $\mathrm{MDH}$ & $465 \mathrm{~F}$ & 13 & Dec 0 & 06 & DWR \\
\hline Styrene & $<0.5$ & $\mathrm{ug} / \mathrm{L}$ & 0.5 & SW802I & $\mathrm{MDH}$ & $465 \mathrm{~F}$ & 13 & Dec 0 & 06 & DWR \\
\hline n-Propylbenzene & $<0.8$ & $\mathrm{ug} / \mathrm{L}$ & 0.8 & SW8021 & $\mathrm{MDH}$ & $465 \mathrm{~F}$ & 13 & Dec 0 & 06 & DWR \\
\hline Bromobenzene & $<0.5$ & $\mathrm{ug} / \mathrm{L}$ & 0.5 & SW802I & $\mathrm{MDH}$ & $465 \mathrm{~F}$ & 13 & Dec 0 & 06 & DWR \\
\hline 2-Chlorotoluene & $<0.8$ & $\mathrm{ug} / \mathrm{L}$ & 0.8 & SWB02I & $\mathrm{MDH}$ & $465 \mathrm{~F}$ & 13 & Dec a & 06 & DWR \\
\hline 1,3,5-Trimethylbenzene & $<0.9$ & $\mathrm{ug} / \mathrm{ta}$ & 0.9 & SW8021 & $\mathrm{MDH}$ & $465 \mathrm{~F}$ & 13 & Dec 0 & 06 & DWR \\
\hline 4-Chlorotoluene & $<0.6$ & $\mathrm{ug} / \mathrm{L}$ & 0.6 & SW8021 & $\mathrm{MDH}$ & $465 \mathrm{~F}$ & 13 & Dec 0 & 06 & DWR \\
\hline t-Butylbenzene & $<0.6$ & $\mathrm{ug} / \mathrm{L}$ & 0.6 & SW8021 & $\mathrm{MDH}$ & $465 \mathrm{~F}$ & 13 & Dec 0 & 06 & DWR \\
\hline 1,2,4-Trimethylbenzene & $<0.7$ & $\mathrm{ug} / \mathrm{L}$ & 0.7 & SW802I & $\mathrm{MDH}$ & $465 \mathrm{~F}$ & 13 & Dec 0 & 06 & DWR \\
\hline sec-Butylbenzene & $<0.7$ & $\mathrm{ug} / \mathrm{L}$ & 0.7 & SWB 021 & $\mathrm{MDH}$ & $465 \mathrm{~F}$ & 13 & Dec 0 & 06 & DWR \\
\hline p-Isopropyl toluene & $<0.7$ & $\mathrm{ug} / \mathrm{L}$ & 0.7 & SWB 021 & $\mathrm{MDH}$ & $465 \mathrm{~F}$ & 13 & Dec 0 & 06 & DWR \\
\hline n-Butylbenzene & $<0.7$ & $\mathrm{ug} / \mathrm{L}$ & 0.7 & SW8021 & $\mathrm{MDH}$ & $465 \mathrm{~F}$ & 13 & Dec 0 & 06 & DWR \\
\hline 1,2-Dibromo-3-chloropropane & $<1$ & $\mathrm{ug} / \mathrm{L}$ & 1 & SW8021 & MDH & $465 \mathrm{~F}$ & 13 & Dec 0 & 06 & DWR \\
\hline 1,2,4-Trichlorobenzene & $<0.5$ & $\mathrm{ug} / \mathrm{L}$ & 0.5 & SW8 021 & $\mathrm{MDH}$ & $465 \mathrm{~F}$ & 13 & Dec 0 & 06 & DWR \\
\hline Hexachlorobutadiene & $<0.7$ & $\mathrm{ug} / \mathrm{L}$ & 0.7 & SWB 021 & $\mathrm{MDH}$ & $465 \mathrm{~F}$ & 13 & Dec 0 & 06 & DWR \\
\hline Naphthalene & $<1$ & $\mathrm{ug} / \mathrm{I}$ & 1 & SW8 021 & $\mathrm{MDH}$ & $465 \mathrm{~F}$ & 13 & Dec 0 & 06 & DWR \\
\hline 1,2,3-Trichlorobenzene & $<0.6$ & $\mathrm{ug} / \mathrm{L}$ & 0.6 & SW8021 & $\mathrm{MDH}$ & $465 \mathrm{~F}$ & 13 & Dec 0 & 06 & DWR \\
\hline GRO (TPH) & $<0.2$ & $\mathrm{mg} / \mathrm{L}$ & 0.2 & $8015 \mathrm{~B} / \mathrm{C}$ & DA1 & & 8 & Dec 0 & 06 & RDC \\
\hline
\end{tabular}

GC VOC Sample $\mathrm{pH}<2$

1-CHLORO-4-FLUOROBENZENE (SURROGATE) RECOVERY： $100 \%$

BTEX/GRO Sample pH $<2$

BTEX SURROGATE RECOVERY: $104 \frac{2}{6}$

GRO SURROGATE RECOVERY: $95 \%$
Page: $\quad 2$ of 2

Report Date: 15 Dec 06

Lab Number: 06-A54133

Work Order \#:82-2656

Account \#: 007033

Sample Matrix: GROUNDWATER

Date Sampled: 6 Dec 06 13:30

Date Received: 8 Dec 06

PO \#: CSI WATFORD

Temp at Receipt: $6.0 \mathrm{C}$ 


\author{
JARDA SOLC \\ ENERGY \& ENVIRONMENTAL RESEARCH CTR \\ UNIVERSITY OF NORTH DAKOTA \\ GRAND FORKS ND 58203
}

\section{Sample Description: $M W-9$}

W5634

\author{
Page: 1 of 2 \\ Report Date: 15 Dec 06 \\ Lab Number: 06-A54134 \\ work Order \#:82-2656 \\ Account \#: 007033 \\ Sample Matrix: GROUNDWATER \\ Date sampled: 6 Dec 06 14:30 \\ Date Received: 8 Dec 06 \\ PO \#: CSI WATFORD \\ Temp at Receipt: $6.0 \mathrm{C}$
}

As Received

Method

Method

Reference

Date

Analyst

Chloroethane
Chloromethane
Bromomethane

Bromomethane

$<0.7 \quad \mathrm{ug} / \mathrm{L}$

RL

SWB $021 \mathrm{MDH} 465 \mathrm{~F}$

SW8021 MDH 465F

SW8021 MDH $465 \mathrm{~F}$

SW8021 MDH $465 \mathrm{~F}$

SW8021 MDH $465 \mathrm{~F}$

SW8021 MDH $465 \mathrm{~F}$

SW8021 MDH 465F

SW8021 MDH $465 \mathrm{~F}$

SW8021 MDH 465F

SW8021 MDH $465 \mathrm{~F}$

SW8021 MDH $465 \mathrm{~F}$

SW8021 MDH 465F

SW8021 MDH 465F

SW8021 MDH 465F

SW8021 MDH 465F

SW8021 MDH $465 \mathrm{~F}$

SW8021 MDH $465 \mathrm{~F}$

SW8021 MDH 465F

SW8021 MDH $465 F$

SW8021 MDH $465 \mathrm{~F}$

SWBO21 MDH $465 \mathrm{~F}$

SW8021 MDH $465 \mathrm{~F}$

SW8021 MDH $465 \mathrm{~F}$

SW8021 MDH $465 \mathrm{~F}$

SW8021 MDH $465 F$

SW8021 MDH 465F

SW8021 MDH $465 \mathrm{~F}$

SW8021 MDH 465F

SW8021 MDH 465F

SW8021 MDH $465 \mathrm{~F}$

SW8021 MDH $465 \mathrm{~F}$

SW8021 MDH 465F

SW8021 MDH 465F

SW8021 MDH $465 \mathrm{~F}$

SW8021 MDH 465F

SW8021 MDH 465F

SW8021 MDH 465F

SW8021 MDH 465F

SW8021 MDH $465 \mathrm{~F}$

SW8021 MDH $465 \mathrm{~F}$

SW8021 MDH $465 \mathrm{~F}$

\begin{tabular}{|c|c|c|c|}
\hline 13 & DeC & 06 & DWR. \\
\hline 13 & DeC & 06 & DWR \\
\hline 13 & Dec & 06 & DWR \\
\hline 13 & Dec & 06 & DWR \\
\hline 13 & Dec & 06 & DWR \\
\hline 13 & DeC & 06 & DWR \\
\hline 13 & Dec & 06 & DWR \\
\hline 13 & Dec & 06 & DWR \\
\hline 13 & Dec & 06 & DWR \\
\hline 13 & Dec & 06 & DWR \\
\hline 13 & Dec & 06 & DWR \\
\hline 13 & Dec & 06 & DWR \\
\hline 13 & Dec & 06 & DWR \\
\hline 13 & Dec & 06 & DWR \\
\hline 13 & Dec & 06 & DWR \\
\hline 13 & Dec & 06 & DWR \\
\hline 13 & Dec & 06 & DWR \\
\hline 13 & Dec & 06 & DWR \\
\hline 13 & Dec & 06 & DWR \\
\hline 13 & Dec & 06 & DWR \\
\hline 13 & Dec & 06 & DWR \\
\hline 13 & Dec & 06 & DWR \\
\hline 13 & Dec & 06 & DWR \\
\hline 13 & Dec & 06 & DWR \\
\hline 13 & Dec & 06 & DWR \\
\hline 13 & Dec & 06 & DWR \\
\hline 13 & Dec & 06 & DWR \\
\hline 13 & Dec & 06 & DWR \\
\hline 13 & Dec & 06 & DWR \\
\hline 13 & Dec & 06 & DWR \\
\hline 13 & Dec & 06 & DWR \\
\hline 13 & DeC & 06 & DWR \\
\hline 13 & Dec & 06 & DWR \\
\hline 13 & Dec & 06 & DWR \\
\hline 13 & DeC & 06 & DWR \\
\hline 13 & Dec & 06 & DWR \\
\hline 13 & Dec & 06 & DWR \\
\hline 13 & Dec & 06 & DWR \\
\hline 13 & Dec & 06 & DWR \\
\hline 13 & DeC & 06 & DWR \\
\hline 13 & Dec & 06 & DWR \\
\hline
\end{tabular}

$m-x y l e n e$ and $p-x y l e n e$

o-xylene

$<5$

$<0.3$

$\mathrm{ug} / \mathrm{L}$

$\mathrm{ug} / \mathrm{L}$ 
JARDA SOLC

ENERGY \& ENVIRONMENTAL RESEARCH CTR UNIVERSITY OF NORTH DAKOTA

GRAND FORKS ND 58203

Sample Description: MW-9

W5 634
Page: 2 of 2

Report Date: 15 Dec 06

Lab Number: $06-$ A54134

Work Order \#: 82-2656

Account \#: 007033

Sample Matrix: GROUNDWATER

Date Sampled: 6 Dec 06 14:30

Date Received: 8 Dec 06

PO \#: CSI WATFORD

Temp at Receipt: $6.0 \mathrm{C}$

\begin{tabular}{|c|c|c|c|c|c|c|c|c|c|c|}
\hline \multirow[b]{2}{*}{ Cumene } & \multicolumn{2}{|c|}{$\begin{array}{l}\text { As Received } \\
\text { Result }\end{array}$} & \multirow{2}{*}{$\begin{array}{l}\text { Method } \\
\text { RL. } \\
0.7\end{array}$} & \multicolumn{3}{|c|}{$\begin{array}{l}\text { Method } \\
\text { Reference }\end{array}$} & \multicolumn{3}{|c|}{$\begin{array}{l}\text { Date } \\
\text { Analyzed }\end{array}$} & \multirow{2}{*}{$\begin{array}{l}\text { Analyst } \\
\text { DWR }\end{array}$} \\
\hline & $<0.7$ & $\mathrm{ug} / \mathrm{L}$ & & SW8021 & $\mathrm{MDH}$ & $465 \mathrm{~F}$ & 13 & Dec & 06 & \\
\hline $1,1,1,2$-Tetrachloroethane & $<0.5$ & $\mathrm{ug} / \mathrm{L}$ & 0.5 & SW8021 & MDH & $465 \mathrm{~F}$ & 13 & Dec & 06 & DWR \\
\hline 1.1-Dichloropropene & $<0.5$ & $u g / 1$ & 0.5 & SW8021 & $\mathrm{MDH}$ & $465 \mathrm{~F}$ & 13 & Dec & 06 & DWR \\
\hline Dichlorof I uoromethane & $<0.5$ & $u g / L$ & 0.5 & SW8021 & $\mathrm{MDH}$ & $465 \mathrm{~F}$ & 13 & Dec & 06 & DWR \\
\hline Trichlorotrifluoroethane & $<0.9$ & $\mathrm{ug} / \mathrm{L}$ & 0.9 & SW8021 & $\mathrm{MDH}$ & $465 \mathrm{~F}$ & 13 & Dec & 06 & DWR \\
\hline Ethyl Ether & $<0.8$ & $\mathrm{ug} / \mathrm{L}$ & 0.8 & SW8021 & $\mathrm{MDH}$ & $465 \mathrm{~F}$ & 13 & Dec & 06 & DWR \\
\hline Acetone & $<10$ & $u g / L$ & 10 & SW8021 & $\mathrm{MDH}$ & $465 \mathrm{~F}$ & 13 & Dec & 06 & DWR \\
\hline Dibromomethane & $<0.7$ & $\mathrm{ug} / \mathrm{L}$ & 0.7 & SW8021 & $\mathrm{MDH}$ & $465 \mathrm{~F}$ & 13 & Dec & 06 & DWR \\
\hline 2,2-Dichloropropane & $<1$ & $\mathrm{ug} / \mathrm{L}$ & 1 & SW8021 & $\mathrm{MDH}$ & $465 \mathrm{~F}$ & 13 & Dec & 06 & DWR \\
\hline Bromochloromethane & $<0.6$ & ug/I. & 0.6 & SW8021. & $\mathrm{MDH}$ & $465 \mathrm{~F}$ & 13 & Dec & 06 & DWR \\
\hline Methyl tert-butyl Ether & $<0.6$ & $\mathrm{ug} / \mathrm{L}$ & 0.6 & SW8021 & $\mathrm{MDH}$ & $465 \mathrm{~F}$ & 13 & Dec & 06 & DWR \\
\hline Styrene & $<0.5$ & $\mathrm{ug} / \mathrm{L}$ & 0.5 & SW8021 & $\mathrm{MDH}$ & $465 \mathrm{~F}$ & 13 & Dec & 06 & DWR \\
\hline n-Propylbenzene & $<0.8$ & $\mathrm{ug} / \mathrm{L}$ & 0.8 & SW8021 & MDH & $465 \mathrm{~F}$ & 13 & Dec & 06 & DWR \\
\hline Bromobenzene & $<0.5$ & $\mathrm{ug} / \mathrm{L}$ & 0.5 & $5 W 8021$ & $\mathrm{MDH}$ & $465 \mathrm{~F}$ & 13 & Dec & 06 & DWR \\
\hline 2-Chlorotoluene & $<0.8$ & $\mathrm{ug} / \mathrm{L}$ & 0.8 & SW8021 & $\mathrm{MDH}$ & $465 \mathrm{~F}$ & 13 & Dec & 06 & DWR \\
\hline $1,3,5$-Trimethyl benzene & $<0.9$ & $\mathrm{ug} / \mathrm{L}$ & 0.9 & SW8021 & $\mathrm{MDH}$ & $465 \mathrm{~F}$ & 13 & DeC & 06 & DWR \\
\hline 4-Chlorotoluene & $<0.6$ & $\mathrm{ug} / \mathrm{L}$ & 0.6 & SW8021 & $\mathrm{MDH}$ & $465 \mathrm{~F}$ & 13 & Dec & 06 & DWR \\
\hline t-Butylbenzene & $<0.6$ & $\mathrm{ug} / \mathrm{L}$ & 0.6 & SW8021 & MDH & $465 \mathrm{~F}$ & 13 & Dec & 06 & DWR \\
\hline $1,2,4$-Trimethyl benzene & $<0.7$ & $\mathrm{ug} / \mathrm{L}$ & 0.7 & SWBO21 & $\mathrm{MDH}$ & $465 \mathrm{~F}$ & 13 & Dec & 06 & DWR \\
\hline sec-Butylbenzene & $<0.7$ & $\mathrm{ug} / \mathrm{L}$ & 0.7 & SW8021 & MDH & $465 \mathrm{~F}$ & 13 & Dec & 06 & DWR \\
\hline p-Isopropyltoluene & $<0.7$ & $\mathrm{ug} / \mathrm{L}$ & 0.7 & SWBO21 & $\mathrm{MDH}$ & $465 \mathrm{~F}$ & 13 & Dec & 06 & DWR \\
\hline n-Butylbenzene & $<0.7$ & $u g / L$ & 0.7 & SW8021 & $\mathrm{MDH}$ & $465 \mathrm{~F}$ & 13 & Dec & 06 & DWR \\
\hline 1.2-Dibromo-3-chloropropane & $<1$ & $\mathrm{ug} / \mathrm{L}$ & 1 & SWB021 & MDH & $465 \mathrm{~F}$ & 13 & Dec & 06 & DWR \\
\hline $1,2,4-\operatorname{Trichlorobenzene}$ & $<0.5$ & $\mathrm{ug} / \mathrm{L}$ & 0.5 & SW8021 & $\mathrm{MDH}$ & $465 \mathrm{~F}$ & 13 & Dec & 06 & DWR \\
\hline Hexachlorobutadiene & $<0.7$ & $\mathrm{ug} / \mathrm{L}$ & 0.7 & SW8021 & $\mathrm{MDH}$ & $465 \mathrm{~F}$ & 13 & Dec & 06 & DWR \\
\hline Naphthalene & $<1$ & $\mathrm{ug} / \mathrm{L}$ & 1. & SW8021 & $\mathrm{MDH}$ & $465 \mathrm{~F}$ & 13 & Dec & 06 & DWR \\
\hline 1,2,3-Trichlorobenzene & $<0.6$ & $\mathrm{ug} / \mathrm{L}$ & 0.6 & SW8021 & $\mathrm{MDH}$ & $465 \mathrm{~F}$ & 13 & Dec & 06 & DWR \\
\hline GRO (TPH) & $<0.2$ & $\mathrm{mg} / \mathrm{L}$ & 0.2 & $8015 \mathrm{~B} / \mathrm{C}$ & DAI & & $B$ & Dec & 06 & RDC \\
\hline
\end{tabular}

GC VOC Sample $\mathrm{pH}<2$

1-CHLORO-4-FLUOROBENZENE (SURROGATE) RECOVERY: $100 \%$

BTEX/GRO Sample $\mathrm{pH}<2$

BTEX SURROGATE RECOVERY: $102 \%$

GRO SURROGATE RECOVERY: $96 \%$ 
JARDA SOLC

ENERGY \& ENVIRONMENTAL RESEARCH CTR

UNIVERSITY OF NORTH DAKOTA

GRAND FORKS ND 58203

Sample Description: MW-11

W5637
Page: 1 of 2

Report Date: 15 Dec 06

Lab Number: $06-A 54137$

work Order \#: $82-2656$

Account \#: 007033

Sample Matrix: GROUNDWATER

Date Sampled: 6 Dec 06 10:15

Date Received: 8 Dec 06

PO \# : CSI WATFORD

Temp at Receipt: $6.0 \mathrm{C}$

\begin{tabular}{|c|c|c|c|c|c|c|c|c|c|}
\hline \multirow{2}{*}{ Chloroethane } & \multicolumn{2}{|c|}{$\begin{array}{l}\text { As Received } \\
\text { Result }\end{array}$} & \multirow{2}{*}{$\begin{array}{l}\text { Method } \\
\text { RL } \\
0.7\end{array}$} & \multicolumn{3}{|c|}{$\begin{array}{l}\text { Method } \\
\text { Reference }\end{array}$} & \multicolumn{2}{|c|}{$\begin{array}{l}\text { Date } \\
\text { Analyzed }\end{array}$} & \multirow{2}{*}{$\begin{array}{l}\text { Analyst } \\
\text { DWR }\end{array}$} \\
\hline & $<700 \#$ & $\mathrm{ug} / \mathrm{L}$ & & SWB 021 & $\mathrm{MDH}$ & $465 \mathrm{~F}$ & 14 & Dec 06 & \\
\hline Chloromethane & $<1000 \#$ & $\mathrm{ug} / \mathrm{L}$ & 1 & SW8 021 & $\mathrm{MDH}$ & $465 \mathrm{~F}$ & 14 & Dec 06 & DWR \\
\hline Bromomethane & $<700 \#$ & $\mathrm{ug} / \mathrm{L}$ & 0.7 & SW8021 & MDH & $465 \mathrm{~F}$ & 14 & Dec 06 & DWR \\
\hline Dichlorodif Iuorome thane & $<900 \#$ & $\mathrm{ug} / \mathrm{L}$ & 0.9 & SW8021 & $\mathrm{MDH}$ & $465 \mathrm{~F}$ & 14 & Dec 06 & DWR \\
\hline vinyl Chloride & $<500 \#$ & ug/L & 0.5 & SW8021 & $\mathrm{MDH}$ & $465 \mathrm{~F}$ & 1.4 & Dec 06 & DWR \\
\hline Methylene Chloride & $<700 \#$ & $\mathrm{ug} / \mathrm{L}$ & 0.7 & SW8021 & $\mathrm{MDH}$ & $465 \mathrm{~F}$ & 14 & Dec 06 & DWR \\
\hline Trichlorofluoromethane & $<900 \#$ & $\mathrm{ug} / \mathrm{L}$ & 0.9 & SW8021 & $\mathrm{MDH}$ & $465 \mathrm{~F}$ & 14 & Dec 06 & DWR \\
\hline 1,1-Dichloroethene & $<500 \#$ & $\mathrm{ug} / \mathrm{L}$ & 0.5 & SW8021 & MDH & $465 \mathrm{~F}$ & 14 & Dec 06 & DWR \\
\hline 1,1-Dichloroethane & $<800 \#$ & $\mathrm{ug} / \mathrm{L}$ & 0.8 & SW8021 & $\mathrm{MDH}$ & $465 \mathrm{~F}$ & 14 & Dec 06 & DWR \\
\hline trans $-1,2-$ Dichloroethene & $<500 \#$ & $\mathrm{ug} / \mathrm{L}$ & 0.5 & SW8021 & MDH & $465 \mathrm{~F}$ & 14 & Dec 06 & DWR \\
\hline Chloroform & $<800 \#$ & $\mathrm{ug} / \mathrm{L}$ & 0.8 & SW8021 & MDH & $465 \mathrm{~F}$ & 14 & Dec 06 & DWR \\
\hline 1.2-Dichloroethane & 2466 & $\mathrm{ug} / \mathrm{L}$ & 0.5 & 5W8021 & $\mathrm{MDH}$ & $465 \mathrm{~F}$ & 14 & Dec 06 & DWR \\
\hline 1,1,1-Trichloroethane & $<900$ 茫 & $\mathrm{ug} / \mathrm{L}$ & 0.9 & Sw8021 & $\mathrm{MDH}$ & $465 \mathrm{~F}$ & 14 & Dec 06 & DWR \\
\hline Carbon Tetrachloride & $<800 \#$ & $\mathrm{ug} / \mathrm{L}$ & 0.8 & SW8021 & $\mathrm{MDH}$ & $465 \mathrm{~F}$ & 14 & Dec 06 & DWR \\
\hline Bromodichlorome thane & $<500 \#$ & $\mathrm{ug} / \mathrm{L}$ & 0.5 & SWBO21 & $\mathrm{MDH}$ & $465 \mathrm{~F}$ & 14 & Dec 06 & DWR \\
\hline 1.2-Dichloropropane & $<700 \#$ & $\mathrm{ug} / \mathrm{L}$ & 0.7 & SW802I & $\mathrm{MDH}$ & $465 \mathrm{~F}$ & 14 & Dec 06 & DWR \\
\hline trans-1.3-Dichloropropene & $<500 \#$ & $\mathrm{ug} / \mathrm{I}$ & 0.5 & SW8021 & $\mathrm{MDH}$ & $465 \mathrm{~F}$ & 14 & Dec 06 & DWR \\
\hline Trichloroethene & $<500 \mathrm{\#}$ & $\mathrm{ug} / \mathrm{L}$ & 0.5 & SWBO21 & $\mathrm{MDH}$ & $465 \mathrm{~F}$ & 14 & Dec 06 & DWR \\
\hline Chlorodibromome thane & $<500$ \# & $\mathrm{ug} / \mathrm{L}$ & 0.5 & SW8021 & $\mathrm{MDH}$ & $465 \mathrm{~F}$ & 14 & Dec 06 & DWR \\
\hline 1,1,2-Trichloroethane & $<600$ \# & $\mathrm{ug} / \mathrm{L}$ & 0.6 & SW8021 & $\mathrm{MDH}$ & $465 \mathrm{~F}$ & 14 & Dec 06 & DWR \\
\hline cis-1,3-Dichloropropene & $<500 \#$ & $\mathrm{ug} / \mathrm{L}$ & 0.5 & SW8021 & $\mathrm{MDH}$ & $465 \mathrm{~F}$ & 14 & Dec 06 & DWR \\
\hline Bromoform & $<1000$ & $\mathrm{ug} / \mathrm{t}$ & 1 & SW8021 & $\mathrm{MDH}$ & $465 \mathrm{~F}$ & 14 & Dec 06 & DWR \\
\hline $1,1,2,2$-Tetrachloroe thane & $<500 \#$ & $\mathrm{ug} / \mathrm{L}$ & 0.5 & SW8021 & $\mathrm{MDH}$ & $465 \mathrm{~F}$ & 14 & Dec 06 & DWR \\
\hline Tetrachloroethene & $<700 \#$ & $\mathrm{ug} / \mathrm{L}$ & 0.7 & SW8021 & $\mathrm{MDH}$ & $465 \mathrm{~F}$ & 14 & Dec 06 & DWR \\
\hline Chlorobenzene & $<600 \#$ & $\mathrm{ug} / \mathrm{L}$ & 0.6 & SWB 021 & $\mathrm{MDH}$ & $465 \mathrm{~F}$ & 14 & Dec 06 & DWR \\
\hline Benzene & 18790 & $\mathrm{ug} / \mathrm{L}$ & 0.5 & SW8021 & MDH & $465 \mathrm{~F}$ & 14 & Dec 06 & DWR \\
\hline Toluene & 1008 & $\mathrm{ug} / \mathrm{L}$ & 0.6 & SW8021 & $\mathrm{MDH}$ & $465 \mathrm{~F}$ & 14 & Dec 06 & DWR \\
\hline Ethyl Benzene & 2125 & $\mathrm{ug} / \mathrm{L}$ & 0.6 & SWB 021 & $\mathrm{MDH}$ & $465 \mathrm{~F}$ & 14 & Dec 06 & DWR \\
\hline 1.2-Dichlorobenzene & $<500 \#$ & $\mathrm{ug} / \mathrm{L}$ & 0.5 & SW8021 & $\mathrm{MDH}$ & $465 \mathrm{~F}$ & 14 & Dec 06 & DWR \\
\hline 1.3-Dichlorobenzene & $<500 \#$ & $\mathrm{ug} / \mathrm{L}$ & 0.5 & SW8021 & MDH & $465 \mathrm{~F}$ & 14 & Dec 06 & DWR \\
\hline 1,4-Dichlorobenzene & $<500 \#$ & $\mathrm{ug} / \mathrm{L}$ & 0.5 & SW8021 & MDH & $465 \mathrm{~F}$ & 14 & Dec 06 & DWR \\
\hline cis-1,2-Dichloroethene & $<500 \#$ & $\mathrm{ug} / \mathrm{L}$ & 0.5 & SW8021 & MDH & $465 \mathrm{~F}$ & 14 & Dec 06 & DWR \\
\hline 1,3-Dichloropropane & $<600 \#$ & $\mathrm{ug} / \mathrm{L}$ & 0.6 & SW8021 & MDH & $465 \mathrm{~F}$ & 14 & Dec 06 & DWR \\
\hline $1,2,3$-Trichloropropane & $<500 \#$ & $\mathrm{ug} / \mathrm{L}$ & 0.5 & SW8021 & MDH & $465 \mathrm{~F}$ & 14 & Dec 06 & DWR \\
\hline Allyl Chloride & $<700$ \# & $\mathrm{ug} / \mathrm{L}$ & 0.7 & SW8021 & MDH & $465 \mathrm{~F}$ & 14 & Dec 06 & DWR \\
\hline 1,2-Dibromoethane & $<500 \#$ & $\mathrm{ug} / \mathrm{L}$ & 0.5 & SW8021 & $\mathrm{MDH}$ & $465 \mathrm{~F}$ & 14 & Dec 06 & DWR \\
\hline Methyl Ethyl Ketone & $<5000 \#$ & $\mathrm{ug} / \mathrm{L}$ & 5 & SW802I & $\mathrm{MDH}$ & $465 \mathrm{~F}$ & 14 & Dec 06 & DWR \\
\hline Methyl Isobutyl Ketone & $<1400$ & $\mathrm{ug} / \mathrm{L}$ & 1.4 & SW8021 & MDH & $465 \mathrm{~F}$ & 14 & Dec 06 & DWR \\
\hline Tetrahydrofuran & $<5000 \#$ & $\mathrm{ug} / \mathrm{L}$ & 5 & SWB021 & $\mathrm{MDH}$ & $465 \mathrm{~F}$ & 14 & Dec 06 & DWR \\
\hline $\mathrm{m}$-xylene and p-xylene & 2534 & $\mathrm{ug} / \mathrm{L}$ & 0.7 & SW8021 & MDH & $465 \mathrm{~F}$ & 14 & Dec 06 & DWR \\
\hline & & & 0.3 & SW8021 & MDH & $465 \mathrm{~F}$ & 14 & Dec 06 & DWR \\
\hline
\end{tabular}

RL = Reporting Limit 
JARDA SOLC

ENERGY \& ENVIRONMENTAL RESEARCH CTR

UNIVERSITY OF NORTH DAKOTA

GRAND FORKS ND 58203

Sample Description: MW-11

W5637

\author{
Page: 2 of 2 \\ Report Date: 15 Dec 06 \\ Lab Number: 06-A54137 \\ Work Order \#:82-2656 \\ Account \#: 007033 \\ Sample Matrix: GROUNDWATER \\ Date Sampled: 6 Dec 06 10:15 \\ Date Received: 8 Dec 06 \\ PO \# : CSI WATFORD \\ Temp at Receipt: $6.0 \mathrm{C}$
}

\begin{tabular}{|c|c|c|c|c|c|c|c|c|c|}
\hline \multirow[b]{2}{*}{ Cumene } & \multicolumn{2}{|c|}{$\begin{array}{l}\text { As Received } \\
\text { Result }\end{array}$} & \multirow{2}{*}{$\begin{array}{l}\begin{array}{l}\text { Method } \\
\text { RL }\end{array} \\
0.7\end{array}$} & \multicolumn{3}{|c|}{$\begin{array}{l}\text { Method } \\
\text { Reference }\end{array}$} & \multicolumn{2}{|c|}{$\begin{array}{l}\text { Date } \\
\text { Analyzed }\end{array}$} & \multirow{2}{*}{$\frac{\text { Analyst }}{\text { DWR }}$} \\
\hline & $<700 \#$ & $\mathrm{ug} / \mathrm{L}$ & & SW8021 & $\mathrm{MDH}$ & $465 \mathrm{~F}$ & 14 & Dec 06 & \\
\hline $1,1,1,2$ - Tetrachloroethane & $<500 \#$ & $\mathrm{ug} / \mathrm{L}$ & 0.5 & SW802I & MDH & $465 \mathrm{~F}$ & 14 & Dec 06 & DWR \\
\hline 1.1-Dichloropropene & $<500 \#$ & $\mathrm{ug} / \mathrm{L}$ & 0.5 & SW8021 & $\mathrm{MDH}$ & $465 \mathrm{~F}$ & 14 & Dec 06 & DWR \\
\hline Dichlorofluoromethane & $<500 \#$ & $\mathrm{ug} / \mathrm{L}$ & 0.5 & SW8021 & $\mathrm{MDH}$ & $465 \mathrm{~F}$ & 14 & Dec 06 & DWR \\
\hline Trichlorotrifluoroethane & $<900 \#$ & $\mathrm{ug} / \mathrm{L}$ & 0.9 & SW8021 & $\mathrm{MDH}$ & $465 \mathrm{~F}$ & 14 & Dec 06 & DWR \\
\hline Ethyl Ether & $<800 \#$ & $u g / L$ & 0.8 & SW8021 & $\mathrm{MDH}$ & $465 F$ & 14 & Dec 06 & DWR \\
\hline Acetone & $<10000 \#$ & $\mathrm{ug} / \mathrm{L}$ & 10 & Sw8 021 & $\mathrm{MDH}$ & $465 F$ & 14 & Dec 06 & DWR \\
\hline Dibromomethane & $<700 \#$ & $\mathrm{ug} / \mathrm{L}$ & 0.7 & SW8021 & $\mathrm{MDH}$ & $465 F$ & 14 & Dec 06 & DWR \\
\hline 2,2-Dichloropropane & $<1000 \#$ & $\mathrm{ug} / \mathrm{L}$ & 1 & SW8021 & $\mathrm{MDH}$ & $465 \mathrm{~F}$ & 14 & Dec 06 & DWR \\
\hline Bromochloromethane & $<600 \#$ & $\mathrm{ug} / \mathrm{L}$ & 0.6 & SWBO21 & $\mathrm{MDH}$ & $465 F$ & 14 & Dec 06 & DWR \\
\hline Methyl tert-butyl Ether & $<600 \#$ & $\mathrm{ug} / \mathrm{L}$ & 0.6 & SW8021 & $\mathrm{MDH}$ & $465 \mathrm{~F}$ & 14 & Dec 06 & DWR \\
\hline Styrene & $<500$ 柏 & $\mathrm{ug} / \mathrm{L}$ & 0.5 & SW8021 & $\mathrm{MDH}$ & $465 \mathrm{~F}$ & 14 & Dec 06 & DWR \\
\hline n-Propylbenzene & $<800$ \# & $\operatorname{ug} / \mathrm{L}$ & 0.8 & SW8021 & MDH & $465 \mathrm{~F}$ & 14 & Dec 06 & DWR \\
\hline Bromobenzene & $<500 \#$ & $\mathrm{ug} / \mathrm{L}$ & 0.5 & SW8021 & MDH & $465 \mathrm{~F}$ & 14 & Dec 06 & DWR \\
\hline 2-Chlorotoluene & $<800$ 扯 & $\mathrm{ug} / \mathrm{L}$ & 0.8 & SW802I & $\mathrm{MDH}$ & $465 \mathrm{~F}$ & 14 & Dec 06 & DWR \\
\hline $1,3,5$-Trimethylbenzene & $<900 \#$ & $\mathrm{ug} / \mathrm{L}$ & 0.9 & SWB 021 & $\mathrm{MDH}$ & $465 \mathrm{~F}$ & 14 & Dec 06 & DWR \\
\hline 4-Chlorotoluene & $<600 \#$ & $\mathrm{ug} / \mathrm{L}$ & 0.6 & SWB 021 & $\mathrm{MDH}$ & $465 \mathrm{~F}$ & 14 & Dec 06 & DWR \\
\hline $\mathrm{t}$-Butylbenzene & $<600$ \# & $\mathrm{ug} / \mathrm{L}$ & 0.6 & SW8021 & $\mathrm{MDH}$ & $465 \mathrm{~F}$ & 14 & Dec 06 & DWR \\
\hline 1.2,4-Trimethylbenzene & 1232 & $\mathrm{ug} / \mathrm{L}$ & 0.7 & SW8021 & $\mathrm{MDH}$ & $465 \mathrm{~F}$ & 14 & Dec 06 & DWR \\
\hline sec-Butylbenzene & $<700 \#$ & $\mathrm{ug} / \mathrm{L}$ & 0.7 & SW8021 & $\mathrm{MDH}$ & $465 \mathrm{~F}$ & 14 & Dec 06 & DWR \\
\hline p-Isopropyltoluene & $<700 \#$ & $\mathrm{ug} / \mathrm{L}$ & 0.7 & SWBO21 & $\mathrm{MDH}$ & $465 \mathrm{~F}$ & 14 & Dec 06 & DWR \\
\hline n-Butylbenzene & $<700 \#$ & $\mathrm{ug} / \mathrm{L}$ & 0.7 & SW8021 & $\mathrm{MDH}$ & $465 \mathrm{~F}$ & 14 & Dec 06 & DWR \\
\hline 1,2-Dibromo-3-chloropropane & $<1000 \#$ & $\mathrm{ug} / \mathrm{L}$ & 1 & SW8021 & $\mathrm{MDH}$ & $465 \mathrm{~F}$ & 14 & Dec 06 & DWR \\
\hline 1,2,4-Trichlorobenzene & $<500 \#$ & $\mathrm{ug} / \mathrm{L}$ & 0.5 & SW8021 & MDH & $465 \mathrm{~F}$ & 14 & Dec 06 & DWR \\
\hline Hexachlorobutadiene & $<700$ \# & $\mathrm{ug} / \mathrm{L}$ & 0.7 & SW8021 & MDH & $465 \mathrm{~F}$ & 14 & Dec 06 & DWR \\
\hline Naphthalene & $<1000 \#$ & $\mathrm{ug} / \mathrm{L}$ & $I$ & SW8021 & $\mathrm{MDH}$ & $465 \mathrm{~F}$ & 14 & Dec 06 & DWR \\
\hline $1,2,3$-Trichlorobenzene & $<600 \#$ & $\mathrm{ug} / \mathrm{L}$ & 0.6 & SW8021 & $\mathrm{MDH}$ & $465 \mathrm{~F}$ & 14 & Dec 06 & DWR \\
\hline GRO (TPH $)$ & 53.65 & $\mathrm{mg} / \mathrm{L}$ & 0.200 & $8015 \mathrm{~B} / \mathrm{C}$ & OA 1 & & 11 & Dec 06 & $\mathrm{RDC}$ \\
\hline
\end{tabular}

GC VoC sample $p H>2$

1-CHLORO-4-FLUOROBENZENE (SURROGATE) RECOVERY: 99 :

BTEX/GRO Sample pH $>2$

BTEX SURROGATE RECOVERY : $98 \%$

GRO SURROGATE RECOVERY: $107 \%$

GRO(TPH) pattern is characteristic of gasoline.

RL = Reporting Limit 


\author{
JARDA SOLC \\ ENERGY \& ENVIRONMENTAL RESEARCH CTR \\ UNIVERSITY OF NORTH DAKOTA \\ GRAND FORKS ND 58203
}

Sample Description: MW-13

W5638

\author{
Page: 1 of 2 \\ Report Date: 15 Dec 06 \\ Lab Number: 06-A54138 \\ Work Order \#: 82-2656 \\ Account \#: 007033 \\ Sample Matxix: GROUNDWATER \\ Date Sampled: 6 Dec 06 9:30 \\ Date Received: 8 Dec 06 \\ PO \# : CSI WATFORD \\ Temp at Receipt: $6.0 \mathrm{C}$
}

\begin{tabular}{|c|c|c|c|c|c|c|c|c|c|c|c|}
\hline \multirow[b]{2}{*}{ Chloroethane } & \multicolumn{3}{|c|}{$\begin{array}{l}\text { As Received } \\
\text { Result }\end{array}$} & \multirow{2}{*}{$\begin{array}{l}\text { Method } \\
\text { RL } \\
0.7\end{array}$} & \multicolumn{3}{|c|}{$\begin{array}{l}\text { Method } \\
\text { Reference }\end{array}$} & \multicolumn{3}{|c|}{$\begin{array}{l}\text { Date } \\
\text { Analyzed }\end{array}$} & \multirow{2}{*}{$\begin{array}{l}\text { Analyst } \\
\text { DWR }\end{array}$} \\
\hline & $<700 \#$ & $\$$ & $\mathrm{ug} / \mathrm{L}$ & & SW8021 & $\mathrm{MDH}$ & $465 \mathrm{~F}$ & 14 & Dec & 06 & \\
\hline Chloromethane & $<1000$ & $\$$ & $\mathrm{ug} / \mathrm{L}$ & 1 & SW8021 & $\mathrm{MDH}$ & $465 \mathrm{~F}$ & 1.4 & Dec & 06 & DWR \\
\hline Bromomethane & $<700 \#$ & $\#$ & $\mathrm{ug} / \mathrm{L}$ & 0.7 & Sw8021 & $\mathrm{MDH}$ & $465 \mathrm{~F}$ & 14 & Dec & 06 & DWR \\
\hline Dichlorodifluoromethane & $<900 \#$ & $\#$ & $\mathrm{ug} / \mathrm{L}$ & 0.9 & Sw8021 & $\mathrm{MDH}$ & $465 \mathrm{~F}$ & 14 & Dec & 06 & DWR \\
\hline Vinyl Chloride & $<500 \#$ & \# & $\mathrm{ug} / \mathrm{L}$ & 0.5 & SW8021 & MDH & $465 \mathrm{~F}$ & 14 & Dec & 06 & DWR \\
\hline Methylene Chloride & $<700 \#$ & \# & $\mathrm{ug} / \mathrm{L}$ & 0.7 & SW8021 & MDH & $465 \mathrm{~F}$ & 14 & Dec & 06 & DWR \\
\hline Trichlorofluoromethane & $<900 \#$ & \# & $\mathrm{ug} / \mathrm{L}$ & 0.9 & SW8021 & $\mathrm{MDH}$ & $465 \mathrm{~F}$ & 14 & Dec & 06 & DWR \\
\hline 1,1-Dichloroethene & $<500 \#$ & $\#$ & $\mathrm{ug} / \mathrm{L}$ & 0.5 & SW8021 & MDH & $465 \mathrm{~F}$ & 14 & Dec & 06 & DWR \\
\hline 1,1-Dichloroethane & $<800 \#$ & \# & $\mathrm{ug} / \mathrm{L}$ & 0.8 & SWB 021 & MDH & $465 \mathrm{~F}$ & 14 & Dec & 06 & DWR \\
\hline trans-1,2-Dichloroethene & $<500 \#$ & $\#$ & $\mathrm{ug} / \mathrm{L}$ & 0.5 & SW802I & MDH & $465 \mathrm{~F}$ & 14 & Dec & 06 & DWR \\
\hline Chioroform & $<800 \#$ & \# & $\mathrm{ug} / \mathrm{L}$ & 0.8 & SWB 021 & MDH & $465 \mathrm{~F}$ & 14 & Dec & 06 & DWR \\
\hline 1,2-Dichloroethane & 1828 & & $\mathrm{ug} / \mathrm{L}$ & 0.5 & SW8 021 & MDH & $465 \mathrm{~F}$ & 14 & Dec & 06 & DWR \\
\hline 1, 1, 1-Trichloroethane & $<900 \#$ & \# & $\mathrm{ug} / \mathrm{L}$ & 0.9 & SWB 021 & MDH & $465 \mathrm{~F}$ & 14 & Dec & 06 & DWR \\
\hline Carbon Tetrachloride & $<800 \#$ & \# & $\mathrm{ug} / \mathrm{L}$ & 0.8 & SW8021 & $\mathrm{MDH}$ & $465 \mathrm{~F}$ & 14 & Dec & 06 & DWR \\
\hline Bromodichlorome thane & $<500 \#$ & $\#$ & $\mathrm{ug} / \mathrm{L}$ & 0.5 & SW8 021 & $\mathrm{MDH}$ & $465 \mathrm{~F}$ & 14 & Dec & 06 & DWR \\
\hline 1,2-Dichloropropane & $<700 \#$ & \# & $\mathrm{ug} / \mathrm{L}$ & 0.7 & SW8021 & $\mathrm{MDH}$ & $465 \mathrm{~F}$ & 14 & Dec & 06 & DWR \\
\hline trans-1,3-Dichloropropene & $<500 \#$ & \# & $\mathrm{ug} / \mathrm{L}$ & 0.5 & SW8021 & $\mathrm{MDH}$ & $465 F$ & 14 & Dec & 06 & DWR \\
\hline Trichloroethene & $<500 \#$ & $\#$ & $\mathrm{ug} / \mathrm{L}$ & 0.5 & SWB021 & MDH & $465 \mathrm{~F}$ & 14 & Dec & 06 & DWR \\
\hline Chlorodibromome thane & $<500$ & \# & $\mathrm{ug} / \mathrm{L}$ & 0.5 & Sw8021 & $\mathrm{MDH}$ & $465 \mathrm{~F}$ & 14 & Dec & 06 & DWR \\
\hline 1,1,2-Trichloroethane & $<600 \#$ & \# & $\mathrm{ug} / \mathrm{L}$ & 0.6 & SW8021 & $\mathrm{MDH}$ & $465 \mathrm{~F}$ & 14 & Dec & 06 & DWR \\
\hline cis-1,3-Dichloropropene & $<500 \#$ & $\#$ & $\mathrm{ug} / \mathrm{L}$ & 0.5 & SW8021 & $\mathrm{MDH}$ & $465 \mathrm{~F}$ & 14 & Dec & 06 & DWR \\
\hline Bromoform & $<1000$ & $\#$ & $\mathrm{ug} / \mathrm{L}$ & 1 & SW8021 & MDH & $465 \mathrm{~F}$ & 14 & Dec & 06 & DWR \\
\hline 1,1,2,2-Tetrachloroethane & $<500 \#$ & \# & $\mathrm{ug} / \mathrm{L}$ & 0.5 & SW8021 & MDH & $465 \mathrm{~F}$ & 14 & Dec & 06 & DWR \\
\hline Tetrachloroethene & $<700 \#$ & $\#$ & $\mathrm{ug} / \mathrm{L}$ & 0.7 & SW8021 & $\mathrm{MDH}$ & $465 \mathrm{~F}$ & 14 & Dec & 06 & DWR \\
\hline Chlorobenzene & $<600 \#$ & \# & $\mathrm{ug} / \mathrm{L}$ & 0.6 & Sw8021 & $\mathrm{MDH}$ & $465 \mathrm{~F}$ & 14 & Dec & 06 & DWR \\
\hline Benzene & 29370 & & $\mathrm{ug} / \mathrm{L}$ & 0.5 & SW8021 & $\mathrm{MDH}$ & $465 \mathrm{~F}$ & 14 & Dec & 06 & DWR \\
\hline Toluene & 4430 & & $\mathrm{ug} / \mathrm{L}$ & 0.6 & SW8021 & $\mathrm{MDH}$ & $465 \mathrm{~F}$ & 14 & Dec & 06 & DWR \\
\hline Ethyl Benzene & 3814 & & $\mathrm{ug} / \mathrm{L}$ & 0.6 & SW8021 & $\mathrm{MDH}$ & $465 \mathrm{~F}$ & 14 & Dec & 06 & DWR \\
\hline 1,2-Dichlorobenzene & $<500$ & $\#$ & $u g / L$ & 0.5 & SW8021 & $\mathrm{MDH}$ & $465 \mathrm{~F}$ & 14 & Dec & 06 & DWR \\
\hline 1,3-Dichlorobenzene & $<500 \#$ & \# & $\mathrm{ug} / \mathrm{L}$ & 0.5 & SW8021 & MDH & $465 \mathrm{~F}$ & 14 & Dec & 06 & DWR \\
\hline 1, A-Dichlorobenzene & $<500 \#$ & $\#$ & $\mathrm{ug} / \mathrm{L}$ & 0.5 & SW8021 & $\mathrm{MDH}$ & $465 \mathrm{~F}$ & 14 & Dec & 06 & DWR \\
\hline cis-1,2-Dichloroethene & $<500 \#$ & $\#$ & $\mathrm{ug} / \mathrm{L}$ & 0.5 & 5W8021 & MDH & $465 \mathrm{~F}$ & 14 & Dec & 06 & DWR \\
\hline 1,3-Dichloropropane & $=600 \#$ & \# & $\mathrm{ug} / \mathrm{L}$ & 0.6 & SWBO21 & $\mathrm{MDH}$ & $465 \mathrm{~F}$ & 14 & Dec & 06 & DWR \\
\hline 1,2,3-Trichloropropane & $<500 \#$ & \# & $\mathrm{ug} / \mathrm{L}$ & 0.5 & SWB 021 & $\mathrm{MDH}$ & $465 \mathrm{~F}$ & 14 & Dec & 06 & DWR \\
\hline Allyl Chloride & $<700 \#$ & \# & $\mathrm{ug} / \mathrm{L}$ & 0.7 & SW8021 & MDH & $465 \mathrm{~F}$ & 14 & Dec & 06 & DWR \\
\hline 1,2-Dibromoethane & $<500$ 抹 & 拉 & $\mathrm{ug} / \mathrm{L}$ & 0.5 & SW8021 & $\mathrm{MDH}$ & $465 \mathrm{~F}$ & 14 & Dec & 06 & DWR \\
\hline Methyl Ethyl Ketone & $<5000$ & \# & $\mathrm{ug} / \mathrm{L}$ & 5 & Sw8021 & $\mathrm{MDH}$ & $465 \mathrm{~F}$ & 14 & Dec & 06 & DWR \\
\hline Methyl Isobutyl Ketone & $<1400$ & \# & $\mathrm{ug} / \mathrm{L}$ & 1.4 & SW8021 & $\mathrm{MDH}$ & $465 \mathrm{~F}$ & 14 & Dec & 06 & DWR \\
\hline Tetrahydrofuran & $<5000$ & $\#$ & $\mathrm{ug} / \mathrm{L}$ & 5 & SWB 021 & $\mathrm{MDH}$ & $465 \mathrm{~F}$ & 14 & Dec & 06 & DWR \\
\hline m-xylene and $p-x_{y}$ lene & 9538 & & $\mathrm{ug} / \mathrm{L}$ & 0.7 & SW8 021 & $\mathrm{MDH}$ & $465 \mathrm{~F}$ & 14 & Dec & 06 & DWR \\
\hline & & & & & SW8021 & $\mathrm{MDH}$ & $465 \mathrm{~F}$ & 14 & Dec & 06 & DWR \\
\hline
\end{tabular}

$R L=$ Reporting Limit 
JARDA SOLC

ENERGY \& ENVIRONMENTAL RESEARCH CTR UNIVERSITY OF NORTH DAKOTA GRAND FORKS ND 58203

Sample Description: $M W-13$

W5638

\author{
Page: 2 of 2 \\ Report Date: 15 Dec 06 \\ Lab Number: 06-A54138 \\ Work Order \#:82-2656 \\ Account \#: 007033 \\ Sample Matrix: GROUNDWATER \\ Date Sampled: 6 Dec 06 9:30 \\ Date Received: 8 Dec 06 \\ PO \#: CSI WATFORD \\ Temp at Receipt: $6.0 \mathrm{C}$
}

\begin{tabular}{|c|c|c|c|c|c|c|c|c|c|c|c|}
\hline \multirow[b]{2}{*}{ Cumene } & \multicolumn{3}{|c|}{$\begin{array}{l}\text { As Received } \\
\text { Result }\end{array}$} & \multirow{2}{*}{$\begin{array}{l}\text { Method } \\
\text { RL } \\
0.7\end{array}$} & \multicolumn{3}{|c|}{$\begin{array}{l}\text { Method } \\
\text { Reference }\end{array}$} & \multicolumn{3}{|c|}{$\begin{array}{l}\text { Date } \\
\text { Analyzed }\end{array}$} & \multirow{2}{*}{$\begin{array}{l}\text { Analyst } \\
\text { DWR }\end{array}$} \\
\hline & $<$ & $700 \$$ & $\mathrm{ug} / \mathrm{L}$ & & SW8021 & $\mathrm{MDH}$ & $465 \mathrm{~F}$ & 14 & Dec & 06 & \\
\hline $1,1,1,2$-Tetrachloroethane & $<$ & $500 \#$ & $\mathrm{ug} / \mathrm{L}$ & 0.5 & SW8021 & $\mathrm{MDH}$ & $465 \mathrm{~F}$ & 14 & Dec & 06 & DWR \\
\hline 1,1-Dichloropropene & $<$ & $500 \#$ & $\mathrm{ug} / \mathrm{L}$ & 0.5 & SW8021 & $\mathrm{MDH}$ & $465 \mathrm{~F}$ & 14 & Dec & 06 & DWR \\
\hline Dichlorofluoromethane & $<$ & $500 \#$ & $\mathrm{ug} / \mathrm{L}$ & 0.5 & SW8021 & MDH & $465 \mathrm{~F}$ & 14 & Dec & 06 & DWR \\
\hline Trichlorotrifluoroethane & $<$ & $900 \#$ & $u g / L$ & 0.9 & SW8021 & MDH & $465 \mathrm{~F}$ & 14 & Dec & 06 & DWR \\
\hline Ethyl Ether & $<$ & $800 \#$ & $\mathrm{ug} / \mathrm{L}$ & 0.8 & SW8021 & $\mathrm{MDH}$ & $465 \mathrm{~F}$ & 14 & Dec & 06 & DWR \\
\hline Acetone & $<$ & $10000 \#$ & $\mathrm{ug} / \mathrm{L}$ & 10 & SWB 021 & MDH & $465 \mathrm{~F}$ & 14 & Dec & 06 & DWR \\
\hline Dibromomethane & $<$ & $700 \#$ & $\mathrm{ug} / \mathrm{L}$ & 0.7 & SW8021 & MDH & $465 F$ & 14 & Dec & 06 & DWR \\
\hline 2.2-Dichloropropane & $<$ & 1000 & $\mathrm{ug} / \mathrm{L}$ & 1 & SW8021 & MDH & $465 \mathrm{~F}$ & 14 & Dec & 06 & DWR \\
\hline Bromochloromethane & $<$ & $600 \#$ & $\mathrm{ug} / \mathrm{L}$ & 0.6 & SW8021 & MDH & $465 \mathrm{~F}$ & 14 & Dec & 06 & DWR \\
\hline Methyl tert-butyl Ether & $<$ & $600 \#$ & $\mathrm{ug} / \mathrm{L}$ & 0.6 & SW8021 & MDH & $465 \mathrm{~F}$ & 14 & Dec & 06 & DWR \\
\hline Styrene & $<$ & $500 \#$ & $\mathrm{ug} / \mathrm{L}$ & 0.5 & SW8021 & $\mathrm{MDH}$ & $465 \mathrm{~F}$ & 14 & Dec & 06 & DWR \\
\hline n-Propylbenzene & $<$ & $800 \#$ & $\mathrm{ug} / \mathrm{L}$ & 0.8 & SWBO 21 & $\mathrm{MDH}$ & $465 \mathrm{~F}$ & 14 & Dec & 06 & DWR \\
\hline Bromobenzene & $<$ & $500 \#$ & $\mathrm{ug} / \mathrm{L}$ & 0.5 & SW8021 & MDH & $465 \mathrm{~F}$ & 14 & Dec & 06 & DWR \\
\hline 2-Chlorotoluene & $<$ & 800 梢 & $\mathrm{ug} / \mathrm{L}$ & 0.8 & SW8021 & $\mathrm{MDH}$ & $465 \mathrm{~F}$ & 14 & Dec & 06 & DWR \\
\hline $1,3,5$-Trimethylbenzene & $<$ & $900 \#$ & $\mathrm{ug} / \mathrm{L}$ & 0.9 & SW8021. & $\mathrm{MDH}$ & $465 \mathrm{~F}$ & 14 & Dec & 06 & DWR \\
\hline 4-Chlorotoluene & $<$ & $600 \#$ & $\mathrm{ug} / \mathrm{L}$ & 0.6 & SW8021 & $\mathrm{MDH}$ & $465 \mathrm{~F}$ & 14 & Dec & 06 & DWR \\
\hline$t$-Butylbenzene & $<$ & $600 \#$ & $\mathrm{ug} / \mathrm{L}$ & 0.6 & SW8021 & MDH & $465 \mathrm{~F}$ & 14 & Dec & 06 & DWR \\
\hline 1.2,4-Trimethylbenzene & 16 & 74 & $\mathrm{ug} / \mathrm{L}$ & 0.7 & SW8021 & $\mathrm{MDH}$ & $465 \mathrm{~F}$ & 14 & Dec & 06 & DWR \\
\hline sec-Butylbenzene & $<$ & $700 \#$ & $\mathrm{ug} / \mathrm{L}$ & 0.7 & SW8021 & $\mathrm{MDH}$ & $465 F$ & 14 & Dec & 06 & DWR \\
\hline p-Isopropyl toluene & $<$ & $700 \mathrm{H}$ & $u g / L$ & 0.7 & SWBO21 & $\mathrm{MDH}$ & $465 \mathrm{~F}$ & 14 & Dec & 06 & DWR \\
\hline n-Butylbenzene & $<$ & $700 \#$ & $\mathrm{ug} / \mathrm{L}$ & 0.7 & SW8021 & $\mathrm{MDH}$ & $465 \mathrm{~F}$ & 14 & Dec & 06 & DWR \\
\hline 1.2-Dibromo-3-chloropropane & $<$ & $1000 \#$ & $\mathrm{ug} / \mathrm{L}$ & 1 & SW8021 & MDH & $465 \mathrm{~F}$ & 14 & Dec & 06 & DWR \\
\hline 1,2,4-Trichlorobenzene & $<$ & $500 \#$ & $\mathrm{ug} / \mathrm{L}$ & 0.5 & SWB021 & MDH & $465 \mathrm{~F}$ & 14 & Dec & 06 & DWR \\
\hline Hexachlorobutadiene & $<$ & $700 H$ & $\mathrm{ug} / \mathrm{L}$ & 0.7 & SW8021 & $\mathrm{MDH}$ & $465 \mathrm{~F}$ & 14 & Dec & 06 & DWR \\
\hline Naphthalene & $<$ & $1000 \#$ & $\mathrm{ug} / \mathrm{L}$ & 1 & SW8021 & $\mathrm{MDH}$ & $465 \mathrm{~F}$ & 14 & Dec & 06 & DWR \\
\hline 1.2.3-Trichlorobenzene & & 600 \# & $\mathrm{ug} / \mathrm{L}$ & 0.6 & SW8021 & MDH & $465 \mathrm{~F}$ & 14 & Dec & 06 & DWR \\
\hline GRO (TPH) & 11 & 12.7 & $\mathrm{mg} / \mathrm{L}$ & 0.200 & $8015 B / C$ & DA1 & & 11 & Dec & 06 & $\mathrm{RDC}$ \\
\hline
\end{tabular}

GC VOC Sample $\mathrm{pH}<2$

1-CHLORO-4-FLUOROBENZENE (SURROGATE) RECOVERY: $101 \%$

BTEX/GRO Sample pH $<2$

BTEX SURROGATE RECOVERY: $93 \%$

GRO SURROGATE RECOVERY: $114 \%$

GRO(TPH) pattern is characteristic of gasoline.

$R L=$ Reporting Limit

Elevated "Less Than Result" $(<)$ : Due to sample matrix $=$ Due to sample quantity
\#- Due to sample concentration

+ - Due to extract volume 
JARDA SOLC

ENERGY \& ENVIRONMENTAL RESEARCH CTR

UNIVERSITY OF NORTH DAKOTA

GRAND FORKS ND 58203

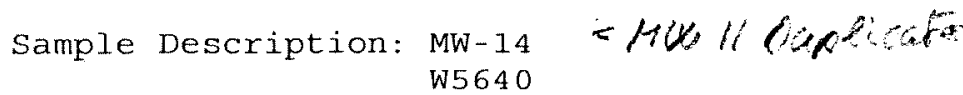

Page: 1 of 2

Report Date: 15 Dec 06

Lab Number: 06-A54140

Work Order \#:82-2656

Account \#: 007033

Sample Matrix: GROUNDWATER

Date Sampled: 6 Dec 06 10:30

Date Received: 8 Dec 06

PO \#: CSI WATFORD

Temp at Receipt: $6.0 \mathrm{C}$

\begin{tabular}{|c|c|c|c|c|c|c|c|c|c|c|c|}
\hline \multirow{2}{*}{ Chloroethane } & \multicolumn{3}{|c|}{$\begin{array}{l}\text { As Received } \\
\text { Result }\end{array}$} & \multirow{2}{*}{$\begin{array}{l}\text { Method } \\
\text { RL } \\
0.7\end{array}$} & \multicolumn{3}{|c|}{$\begin{array}{l}\text { Method } \\
\text { Reference }\end{array}$} & \multicolumn{3}{|c|}{$\begin{array}{l}\text { Date } \\
\text { Analyzed }\end{array}$} & \multirow{2}{*}{$\begin{array}{l}\text { Analyst } \\
\text { DWR }\end{array}$} \\
\hline & $<700$ \# & \# & $\mathrm{ug} / \mathrm{L}$ & & SWB021 & $\mathrm{MDH}$ & $465 \mathrm{~F}$ & 14 & Dec & 06 & \\
\hline Chloromethane & $<1000$ & $\#$ & $\mathrm{ug} / \mathrm{L}$ & 1 & SW8021 & $\mathrm{MDH}$ & $465 \mathrm{~F}$ & 14 & Dec & 06 & DWR \\
\hline Bromomethane & $<700 \#$ & $\#$ & $\mathrm{ug} / \mathrm{L}$ & 0.7 & SWB021 & MDH 4 & $465 \mathrm{~F}$ & 14 & Dec & 06 & DWR \\
\hline Dichlorodifluoromethane & $<900 \#$ & $\#$ & $\mathrm{ug} / \mathrm{L}$ & 0.9 & SW8021 & MDH 4 & $465 \mathrm{~F}$ & 14 & Dec & 06 & DWR \\
\hline Vinyl Chloride & $<500 \#$ & $\#$ & ug/L & 0.5 & SW8021 & MDH 4 & $465 \mathrm{~F}$ & 14 & Dec & 06 & DWR \\
\hline Methylene Chloride & $<700$ & $\#$ & $\mathrm{ug} / \mathrm{L}$ & 0.7 & SW8021 & MDH 4 & $465 \mathrm{~F}$ & 14 & Dec & 06 & DWR \\
\hline Trichiorof Iuoromethane & $<900 \#$ & \# & $\mathrm{ug} / \mathrm{L}$ & 0.9 & SW802I & MDH & $465 \mathrm{~F}$ & 14 & Dec & 06 & DWR \\
\hline 1,1-Dichloroethene & $<500 \#$ & \# & $\mathrm{ug} / \mathrm{L}$ & 0.5 & SW802I & $\mathrm{MDH}$ & $465 \mathrm{~F}$ & 14 & Dec & 06 & DWR \\
\hline 1, 1-Dichloroethane & $<800 \#$ & $\#$ & $\mathrm{ug} / \mathrm{L}$ & 0.8 & Sw8021 & MDH & $465 \mathrm{~F}$ & 14 & Dec & 06 & DWR \\
\hline trans-1,2-Dichloroethene & $<500 \#$ & \# & $\mathrm{ug} / \mathrm{L}$ & 0.5 & SW8021 & $\mathrm{MDH}$ & $465 \mathrm{~F}$ & 1.4 & Dec & 06 & DWR \\
\hline Chloroform & $<800 \#$ & $\#$ & ug/L & 0.8 & SW8021 & $\mathrm{MDH}$ & $465 \mathrm{~F}$ & 14 & Dec & 06 & DWR \\
\hline 1,2-Dichloroethane & 2869 & & $\mathrm{ug} / \mathrm{L}$ & 0.5 & SW8021 & MDH & $465 \mathrm{~F}$ & 14 & Dec & 06 & DWR \\
\hline 1,1,1-Trichloroethane & $<900 \#$ & \# & $\mathrm{ug} / \mathrm{L}$ & 0.9 & SWB021 & $\mathrm{MDH}$ & $465 \mathrm{~F}$ & 14 & Dec & 06 & DWR \\
\hline Carbon Tetrachloride & $<800 \#$ & $\#$ & $\mathrm{ug} / \mathrm{L}$ & 0.8 & SW8021 & $\mathrm{MDH}$ & $465 \mathrm{~F}$ & 14 & Dec & 06 & DWR \\
\hline Bromodichloromethane & $<500 \#$ & \# & $\mathrm{ug} / \mathrm{L}$ & 0.5 & SWB 021 & $\mathrm{MDH}$ & $465 \mathrm{~F}$ & 14 & Dec & 06 & DWR \\
\hline 1,2-Dichloropropane & $<700 \#$ & \# & $\mathrm{ug} / \mathrm{L}$ & 0.7 & SW8021 & MDH & $465 \mathrm{~F}$ & 14 & Dec & 06 & DWR \\
\hline trans-1,3-Dichloropropene & $<500 \#$ & \# & $\mathrm{ug} / \mathrm{L}$ & 0.5 & SW8 021 & $\mathrm{MDH}$ & $465 \mathrm{~F}$ & 14 & Dec & 06 & DWR \\
\hline Trichloroethene & $<500 \#$ & \# & $\mathrm{ug} / \mathrm{L}$ & 0.5 & SW8021 & MDH & $465 \mathrm{~F}$ & 14 & Dec & 06 & DWR \\
\hline Chlorodibromomethane & $<500 \#$ & \# & $\mathrm{ug} / \mathrm{L}$ & 0.5 & SW8021 & $\mathrm{MDH}$ & $465 \mathrm{~F}$ & 14 & Dec & 06 & DWR \\
\hline 1,1,2-Trichloroethane & $<600 \mathrm{H}$ & \# & $\mathrm{ug} / \mathrm{L}$ & 0.6 & SWB021 & $\mathrm{MDH}$ & $465 \mathrm{~F}$ & 14 & Dec & 06 & DWR \\
\hline cis-1,3-Dichloropropene & $<500 \#$ & \# & $\mathrm{ug} / \mathrm{L}$ & 0.5 & SW8021 & $\mathrm{MDH}$ & $465 \mathrm{~F}$ & 14 & Dec & 06 & DWR \\
\hline Bromoform & $<1000$ & 㭅 & $\mathrm{ug} / \mathrm{L}$ & 1 & SW8021 & MDH & $465 \mathrm{~F}$ & 14 & Dec & 06 & DWR \\
\hline $1,1,2,2$-Tetrachloroethane & $<500 \#$ & $\#$ & $\mathrm{ug} / \mathrm{L}$ & 0.5 & SW8021 & $\mathrm{MDH}$ & $465 \mathrm{~F}$ & 14 & Dec & 06 & DWR \\
\hline Tetrachloroethene & $<700 \#$ & \# & $\mathrm{ug} / \mathrm{L}$ & 0.7 & SW8021 & MDH & $465 \mathrm{~F}$ & 14 & Dec & 06 & DWR \\
\hline Chlorobenzene & $<600 \#$ & \# & $\mathrm{ug} / \mathrm{L}$ & 0.6 & Sw8 021 & $\mathrm{MDH}$ & $465 \mathrm{~F}$ & 14 & Dec & 06 & DWR \\
\hline Benzene & 21550 & & $\mathrm{ug} / \mathrm{L}$ & 0.5 & SW8021 & $\mathrm{MDH}$ & $465 \mathrm{~F}$ & 14 & Dec & 06 & DWR \\
\hline Toluene & 1125 & & $\mathrm{ug} / \mathrm{L}$ & 0.6 & SW8 021 & $\mathrm{MDH}$ & $465 \mathrm{~F}$ & 14 & Dec & 06 & DWR \\
\hline Ethyl Benzene & 2406 & & $\mathrm{ug} / \mathrm{L}$ & 0.6 & SW8021 & $\mathrm{MDH}$ & $465 \mathrm{~F}$ & 14 & Dec & 06 & DWR \\
\hline 1.2-Dichlorobenzene & $<500 \#$ & $\#$ & $\mathrm{ug} / \mathrm{L}$ & 0.5 & SW8021 & $\mathrm{MDH}$ & $465 \mathrm{~F}$ & 14 & Dec & 06 & DWR \\
\hline 1.3-Dichlorobenzene & $<500 \#$ & $\#$ & $\mathrm{ug} / \mathrm{L}$ & 0.5 & SW8021 & MDH & $465 \mathrm{~F}$ & 14 & Dec & 06 & DWR \\
\hline 1,4-Dichlorobenzene & $<500 \#$ & \# & $\mathrm{ug} / \mathrm{L}$ & 0.5 & SW8021 & MDH & $465 \mathrm{~F}$ & 14 & Dec & 06 & DWR \\
\hline cis-1,2-Dichloroethene & $<500$ & \# & $\mathrm{ug} / \mathrm{L}$ & 0.5 & SW8021 & MDH & $465 \mathrm{~F}$ & 14 & Dec & 06 & DWR \\
\hline 1,3-Dichloropropane & $<600 \#$ & $\#$ & $\mathrm{ug} / \mathrm{L}$ & 0.6 & SW8021 & $\mathrm{MDH}$ & $465 \mathrm{~F}$ & 14 & Dec & 06 & DWR \\
\hline 1.2,3-Trichloropropane & $<500$ & 护 & $\mathrm{ug} / \mathrm{L}$ & 0.5 & SW8021 & $\mathrm{MDH}$ & $465 \mathrm{~F}$ & 14 & Dec & 06 & DWR \\
\hline Allyl Chloride & $<700$ & 掊 & $\mathrm{ug} / \mathrm{L}$ & 0.7 & SW8021 & $\mathrm{MDH}$ & $465 \mathrm{~F}$ & 14 & Dec & 06 & DWR \\
\hline 1.2-Dibromoethane & $<500$ \# & 邦 & $\mathrm{ug} / \mathrm{L}$ & 0.5 & SW8021 & $\mathrm{MDH}$ & $465 \mathrm{~F}$ & 14 & Dec & 06 & DWR \\
\hline Methyl Ethyl Ketone & $<5000$ & $\#$ & ug/L & 5 & SWB021 & MDH & $465 \mathrm{~F}$ & 14 & Dec & 06 & DWR \\
\hline Methyl Isobutyl Ketone & $<1400$ & $\#$ & $\mathrm{ug} / \mathrm{L}$ & 1.4 & SW8021 & $\mathrm{MDH}$ & $465 \mathrm{~F}$ & 14 & Dec & 06 & DWR \\
\hline Tetrahydrofuran & $<5000$ & $\#$ & $\mathrm{ug} / \mathrm{L}$ & 5 & SW8021 & MDH & $465 \mathrm{~F}$ & 14 & Dec & 06 & DWR \\
\hline$m-x y l e n e$ and $p-x y l e n e$ & 2840 & & $\mathrm{ug} / \mathrm{L}$ & 0.7 & SW8021 & $\mathrm{MDH}$ & $465 \mathrm{~F}$ & 14 & Dec & 06 & DWR \\
\hline o-xylene & $<300 \#$ & $\$$ & $\mathrm{ug} / \mathrm{L}$ & 0.3 & Swg021 & MDH & $465 \mathrm{~F}$ & 14 & Dec & 06 & DWR \\
\hline
\end{tabular}

$R L=$ Reporting Limit 
JARDA SOLC

ENERGY \& ENVIRONMENTAL RESEARCH CTR

UNIVERSITY OF NORTH DAKOTA

GRAND FORKS ND 58203

\section{Sample Description: MW-14}

W5640

\begin{tabular}{|c|c|c|c|c|c|c|c|c|c|c|}
\hline \multirow[b]{2}{*}{ Cumene } & \multicolumn{3}{|c|}{$\begin{array}{l}\text { As Received } \\
\text { Result }\end{array}$} & \multirow{2}{*}{$\begin{array}{l}\text { Method } \\
\text { RL } \\
0.7\end{array}$} & \multicolumn{3}{|c|}{$\begin{array}{l}\text { Method } \\
\text { Reference }\end{array}$} & \multicolumn{2}{|c|}{$\begin{array}{l}\text { Date } \\
\text { Analyzed }\end{array}$} & \multirow{2}{*}{$\begin{array}{l}\text { Analyst } \\
\text { DWR }\end{array}$} \\
\hline & $<$ & $700 \#$ & $\mathrm{ug} / \mathrm{L}$ & & SW8021 & MDH & $465 \mathrm{~F}$ & 14 & Dec 06 & \\
\hline 1,1,1,2-Tetrachloroethane & $<$ & $500 \#$ & $\mathrm{ug} / \mathrm{L}$ & 0.5 & SWB021 & MDH & $465 \mathrm{~F}$ & 14 & Dec 06 & DWR \\
\hline 1,1-Dichloropropene & $<$ & $500 \#$ & $\mathrm{ug} / \mathrm{L}$ & 0.5 & SW802I & MDH & $465 \mathrm{~F}$ & 14 & Dec 06 & DWR \\
\hline Dichlorofluoromethane & $<$ & $500 \#$ & $\mathrm{ug} / \mathrm{L}$ & 0.5 & SW8021 & $\mathrm{MDH}$ & $465 \mathrm{~F}$ & 14 & Dec 06 & DWR \\
\hline Trichlorotrifluoroethane & $<$ & $900 \#$ & $\mathrm{ug} / \mathrm{L}$ & 0.9 & SW802I & $\mathrm{MDH}$ & $465 \mathrm{~F}$ & 14 & Dec 06 & DWR \\
\hline Ethyl Ether & $<$ & $800 \#$ & $\mathrm{ug} / \mathrm{L}$ & 0.8 & SWB021 & $\mathrm{MDH}$ & $465 \mathrm{~F}$ & 14 & Dec 06 & DWR \\
\hline Acetone & $<$ & 10000 & ug/L & 10 & SW8021 & $\mathrm{MDH}$ & $465 \mathrm{~F}$ & 14 & Dec 06 & DWR \\
\hline Dibromomethane & $<$ & 700 & $\mathrm{ug} / \mathrm{L}$ & 0.7 & SW8021 & $\mathrm{MDH}$ & $465 \mathrm{~F}$ & 14 & Dec 06 & DWR \\
\hline 2,2-Dichloropropane & $<$ & $1000 \#$ & $\mathrm{ug} / \mathrm{L}$ & 1 & SW8021 & $\mathrm{MDH}$ & $465 \mathrm{~F}$ & 14 & Dec 06 & DWR \\
\hline Bromochloromethane & $<$ & $600 \#$ & $\mathrm{ug} / \mathrm{L}$ & 0.6 & SW8021 & $\mathrm{MDH}$ & $465 \mathrm{~F}$ & 14 & Dec 06 & DWR \\
\hline Methyl tert-butyl Ether & $<$ & $600 \#$ & $\mathrm{ug} / \mathrm{L}$ & 0.6 & SW8021 & MDH & $465 F$ & 14 & Dec 06 & DWR \\
\hline styrene & $<$ & $500 \#$ & $\mathrm{ug} / \mathrm{L}$ & 0.5 & SW802I & MDH & $465 \mathrm{~F}$ & 14 & Dec 06 & DWR \\
\hline n-Propylbenzene & $<$ & $800 \#$ & $\mathrm{ug} / \mathrm{L}$ & 0.8 & SW8021 & MDH & $465 \mathrm{~F}$ & 14 & Dec 06 & DWR \\
\hline Bromobenzene & $<$ & $500 \#$ & $\mathrm{ug} / \mathrm{L}$ & 0.5 & Sw8021 & $\mathrm{MDH}$ & $465 \mathrm{~F}$ & 14 & Dec 06 & DWR \\
\hline 2-Chlorotoluene & $<$ & $800 \#$ & $\mathrm{ug} / \mathrm{L}$ & 0.8 & SWB 021 & $\mathrm{MDH}$ & $465 \mathrm{~F}$ & 14 & Dec 06 & DWR \\
\hline 1,3,5-Trimethylbenzene & $<$ & $900 \#$ & $\mathrm{ug} / \mathrm{L}$ & 0.9 & SW8 021 & MDH & $465 \mathrm{~F}$ & 14 & Dec 06 & DWR \\
\hline 4-Chlorotoluene & $<$ & $600 \#$ & $\mathrm{ug} / \mathrm{L}$ & 0.6 & SW8021 & $\mathrm{MDH}$ & $465 \mathrm{~F}$ & 14 & Dec 06 & DWR \\
\hline t-ButyIbenzene & & $600 \#$ & $\mathrm{ug} / \mathrm{L}$ & 0.6 & SW8021 & $\mathrm{MDH}$ & $465 \mathrm{~F}$ & 14 & Dec 06 & DWR \\
\hline $1,2,4$-Trimethylbenzene & & 16 & $\mathrm{ug} / \mathrm{L}$ & 0.7 & SW8021 & $\mathrm{MDH}$ & $465 \mathrm{~F}$ & 14 & Dec 06 & DWR \\
\hline sec-Butylbenzene & & $700 \#$ & $\mathrm{ug} / \mathrm{L}$ & 0.7 & SW8021 & MDH & $465 \mathrm{~F}$ & 14 & Dec 06 & DWR \\
\hline p-Isopropyltoluene & $<$ & $700 \#$ & $\mathrm{ug} / \mathrm{L}$ & 0.7 & SW8021 & MDH & $465 \mathrm{~F}$ & 14 & Dec 06 & DWR \\
\hline n-Butylbenzene & & $700 \#$ & $\mathrm{ug} / \mathrm{L}$ & 0.7 & SWB 021 & MDH & $465 \mathrm{~F}$ & 14 & Dec 06 & DWR \\
\hline 1,2-Dibromo-3-chloropropane & & $1000 \#$ & $\mathrm{ug} / \mathrm{L}$ & 1 & SW8021 & $\mathrm{MDH}$ & $465 \mathrm{~F}$ & 14 & Dec 06 & DWR \\
\hline $1,2,4$-Trichlorobenzene & & $500 \#$ & $\mathrm{ug} / \mathrm{L}$ & 0.5 & SW8021 & MDH & $465 \mathrm{~F}$ & 14 & Dec 06 & DWR \\
\hline Hexachlorobutadiene & & $700 \#$ & $\mathrm{ug} / \mathrm{L}$ & 0.7 & SW8021 & $\mathrm{MDH}$ & $465 \mathrm{~F}$ & 14 & Dec 06 & DWR \\
\hline Naphthalene & & $1000 \#$ & $\mathrm{ug} / \mathrm{L}$ & 1 & SW8021 & MDH & $465 \mathrm{~F}$ & 14 & Dec 06 & DWR \\
\hline 1,2,3-Trichlorobenzene & & $600 \mathrm{\#}$ & $\mathrm{ug} / \mathrm{L}$ & 0.6 & SW8021 & $\mathrm{MDH}$ & $465 \mathrm{~F}$ & 14 & Dec 06 & DWR \\
\hline GRO (TPH) & & .22 & $\mathrm{mg} / \mathrm{L}$ & 0.200 & $8015 \mathrm{~B} / \mathrm{C}$ & OAI & & 11 & Dec 06 & RDC \\
\hline
\end{tabular}

GC VoC Sample $\mathrm{pH}>2$

1-CHLORO-4-FLUOROBENZENE (SURROGATE) RECOVERY: $99 \div$

BTEX/GRO Sample $\mathrm{pH}>2$

BTEX SURROGATE RECOVERY: $99 \%$

GRO SURROGATE RECOVERY : $110 \%$

GRO(TPH) pattern is characteristic of gasoline.
Page: 2 of 2

Report Date: 15 Dec 06

Lab Number: $06-A 54140$

work Order \#:82-2656

Account \#: 007033

Sample Matrix: GROUNDWATER

Date Sampled: 6 Dec 06 10:30

Date Received: 8 Dec 06

PO \#: CSI WATFORD

Temp at Receipt: $6.0 \mathrm{C}$

A

$4 \Omega T$ 
JARDA SOLC

ENERGY \& ENVIRONMENTAL RESEARCH CTR

UNIVERSTTY OF NORTH DAKOTA

GRAND FORKS ND 58203

Sample Description: REC-1

W5639
Page: 1 of 2

Report Date: 15 Dec 06

Lab Number: 06-A54139

Work Order \#:82-2656

Account \#: 007033

Sample Matrix: GROUNDWATER

Date Sampled: 6 Dec 06 12:00

Date Received: 8 Dec 06

PO \# : CSI WATFORD

Temp at Receipt: $6.0 \mathrm{C}$

\begin{tabular}{|c|c|c|c|c|c|c|c|c|c|c|}
\hline \multirow[b]{2}{*}{ Chloroethane } & \multicolumn{2}{|c|}{$\begin{array}{l}\text { As Received } \\
\text { Result }\end{array}$} & \multirow{2}{*}{$\begin{array}{l}\text { Method } \\
\text { RL } \\
0.7\end{array}$} & \multicolumn{3}{|c|}{$\begin{array}{l}\text { Method } \\
\text { Reference }\end{array}$} & \multicolumn{3}{|c|}{ Date } & \multirow{2}{*}{$\begin{array}{l}\text { Analyst } \\
\text { DWR }\end{array}$} \\
\hline & $<700 \#$ & $\mathrm{ug} / \mathrm{L}$ & & SW8021 & $\mathrm{MDH}$ & $465 \mathrm{~F}$ & 14 & Dec & 06 & \\
\hline Chloromethane & $<1000 \#$ & $\mathrm{ug} / \mathrm{L}$ & 1 & SW8021 & $\mathrm{MDH}$ & $465 \mathrm{~F}$ & 14 & Dec & 06 & DWR \\
\hline Bromomethane & $<700 \#$ & $\mathrm{ug} / \mathrm{L}$ & 0.7 & SWB 021 & $\mathrm{MDH}$ & $465 \mathrm{~F}$ & 14 & Dec & 06 & DWR \\
\hline Dichlorodifluoromethane & $=900 \#$ & $\mathrm{ug} / \mathrm{I}$ & 0.9 & SWBO21 & $\mathrm{MDH}$ & $465 \mathrm{~F}$ & 14 & Dec & 06 & DWR \\
\hline Vinyl Chloride & $<500 \#$ & $\mathrm{ug} / \mathrm{L}$ & 0.5 & SW8021 & $\mathrm{MDH}$ & $465 \mathrm{~F}$ & 14 & Dec & 06 & DWR \\
\hline Methylene chloride & $<700 \#$ & ug/L & 0.7 & SW8021 & $\mathrm{MDH}$ & $465 \mathrm{~F}$ & 14 & Dec & 06 & DWR \\
\hline Trichlor of luoromethane & $<900$ & $\mathrm{ug} / \mathrm{L}$ & 0.9 & SW8021 & $\mathrm{MDH}$ & $465 \mathrm{~F}$ & 14 & Dec & 06 & DWR \\
\hline 1,1-Dichloroethene & $<500 \#$ & $\mathrm{ug} / \mathrm{L}$ & 0.5 & SW8021 & $\mathrm{MDH}$ & $465 \mathrm{~F}$ & 14 & Dec & 06 & DWR \\
\hline 1, I-Dichloroethane & $<800 \#$ & $\mathrm{ug} / \mathrm{L}$ & 0.8 & SW8021 & $\mathrm{MDH}$ & $465 \mathrm{~F}$ & 14 & Dec & 06 & DWR \\
\hline trans-1,2-Dichloroethene & $<500$ & $\mathrm{ug} / \mathrm{L}$ & 0.5 & SW8021 & $\mathrm{MDH}$ & $465 \mathrm{~F}$ & 14 & Dec & 06 & DWR \\
\hline Chloroform & $<800 \#$ & $\mathrm{ug} / \mathrm{L}$ & 0.8 & SW8021 & $\mathrm{MDH}$ & $465 \mathrm{~F}$ & 14 & Dec & 06 & DWR \\
\hline 1,2-Dichloroethane & 2095 & $\mathrm{ug} / \mathrm{L}$ & 0.5 & SW8021 & $\mathrm{MDH}$ & $465 \mathrm{~F}$ & 14 & Dec & 06 & DWR \\
\hline $1,1,1$-Trichloroethane & $<900$ \# & $\mathrm{ug} / \mathrm{L}$ & 0.9 & SW8021 & $\mathrm{MDH}$ & $465 \mathrm{~F}$ & 14 & Dec & 06 & DWR \\
\hline Carbon Tetrachloride & $<800$ & $\mathrm{ug} / \mathrm{L}$ & 0.8 & SW8021 & $\mathrm{MOH}$ & $465 F$ & 14 & Dec & 06 & DWR \\
\hline Bromodichlorome thane & $<500$ 相 & $\mathrm{ug} / \mathrm{L}$ & 0.5 & SWBO21 & $\mathrm{MDH}$ & $465 F$ & 14 & Dec & 06 & DWR \\
\hline 1,2-Dichloropropane & $<700$ 邦 & $\mathrm{ug} / \mathrm{L}$ & 0.7 & SW8021 & $\mathrm{MDH}$ & $465 \mathrm{~F}$ & 14 & Dec & 06 & DWR \\
\hline trans-1,3-Dichloropropene & $<500$ \# & $\mathrm{ug} / \mathrm{L}$ & 0.5 & SW8021 & $\mathrm{MDH}$ & $465 \mathrm{~F}$ & 14 & Dec & 06 & DWR \\
\hline rrichloroethene & $<500$ 玤 & $\mathrm{ug} / \mathrm{L}$ & 0.5 & SW8021 & MDH & $465 \mathrm{~F}$ & 14 & Dec & 06 & DWR \\
\hline Chlorodibromome thane & $<500$ & $\mathrm{ug} / \mathrm{L}$ & 0.5 & SW8021 & MDH & $465 \mathrm{~F}$ & 14 & Dec & 06 & DWR \\
\hline 1,1,2-Trichloroethane & $<600$ & $\mathrm{ug} / \mathrm{L}$ & 0.6 & SW8021. & MDH & $465 \mathrm{~F}$ & 14 & Dec & 06 & DWR \\
\hline cis-1,3-Dichloropropene & $<500$ \# & $\mathrm{ug} / \mathrm{L}$ & 0.5 & SW8021 & MDH & $465 \mathrm{~F}$ & 14 & Dec & 06 & DWR \\
\hline Bromoform & $<1000 \#$ & $\mathrm{ug} / \mathrm{L}$ & 1 & SW8021 & $\mathrm{MDH}$ & $465 \mathrm{~F}$ & 14 & Dec & 06 & DWR \\
\hline 1,1,2,2-Tetrachloroethane & $<500$ 柿 & $\mathrm{ug} / \mathrm{L}$ & 0.5 & SW8021 & $\mathrm{MDH}$ & $465 \mathrm{~F}$ & 14 & Dec & 06 & DWR \\
\hline Tetrachloroethene & $<700$ \# & $\mathrm{ug} / \mathrm{L}$ & 0.7 & SW8021 & MDH & $465 \mathrm{~F}$ & 14 & Dec & 06 & DWR \\
\hline Chlorobenzene & $<600 \#$ & $\mathrm{ug} / \mathrm{L}$ & 0.6 & SW8021 & MDH & $465 \mathrm{~F}$ & 14 & Dec & 06 & DWR \\
\hline Benzene & 17800 & $\mathrm{ug} / \mathrm{L}$ & 0.5 & SW802I & MDH & $465 \mathrm{~F}$ & 14 & Dec & 06 & DWR \\
\hline Toluene & 862.9 & $\mathrm{ug} / \mathrm{L}$ & 0.6 & SWB 021 & $\mathrm{MDH}$ & $465 \mathrm{~F}$ & 14 & Dec & 06 & DWR \\
\hline Ethyl Benzene & 3206 & $\mathrm{ug} / \mathrm{L}$ & 0.6 & SW8021 & $\mathrm{MDH}$ & $465 \mathrm{~F}$ & 14 & Dec & 06 & DWR \\
\hline 1,2-Dichlorobenzene & $<500 \#$ & $u g / d$ & 0.5 & SW8021 & $\mathrm{MDH}$ & $465 \mathrm{~F}$ & 14 & Dec & 06 & DWR \\
\hline 1,3-Dichlorobenzene & $<500$ \# & $u g / L$ & 0.5 & SW802I & $\mathrm{MDH}$ & $465 \mathrm{~F}$ & 14 & Dec & 06 & DWR \\
\hline 1, 4-Dichlorobenzene & $<500 \#$ & $u g / L$ & 0.5 & SW8021 & MDH & $465 \mathrm{~F}$ & 14 & Dec & 06 & DWR \\
\hline cis-1,2-Dichloroethene & $<500 \#$ & $\mathrm{ug} / \mathrm{I}$ & 0.5 & SW8021 & $\mathrm{MDH}$ & $465 \mathrm{~F}$ & 14 & Dec & 06 & DWR \\
\hline 1,3-Dichloropropane & $<600 \#$ & $\mathrm{ug} / \mathrm{L}$ & 0.6 & SW8 021 & $\mathrm{MDH}$ & $465 \mathrm{~F}$ & 14 & Dec & 06 & DWR \\
\hline $1,2,3$-Trichloropropane & $<500 \#$ & $\mathrm{ug} / \mathrm{L}$ & 0.5 & SW8 021 & $\mathrm{MDH}$ & $465 \mathrm{~F}$ & 14 & Dec & 06 & DWR \\
\hline Allyl Chloride & $<700 \#$ & $\mathrm{ug} / \mathrm{L}$ & 0.7 & SW8021 & MDH & $465 \mathrm{~F}$ & 14 & Dec & 06 & DWR \\
\hline 1,2-Dibromoethane & $<500 \#$ & $\mathrm{ug} / \mathrm{L}$ & 0.5 & SWB 021 & $\mathrm{MDH}$ & $465 F$ & 14 & Dec & 06 & DWR \\
\hline Methyl Ethyl Ketone & $<5000 \#$ & $\mathrm{ug} / \mathrm{L}$ & 5 & SW8021 & $\mathrm{MDH}$ & $465 \mathrm{~F}$ & 14 & Dec & 06 & DWR \\
\hline Methyl Isobutyl ketone & $<1400$ & $\mathrm{ug} / \mathrm{L}$ & 1.4 & SW8021 & $\mathrm{MDH}$ & $465 \mathrm{~F}$ & 14 & Dec & 06 & DWR \\
\hline Tetrahydrofuran & $<5000$ H & $\mathrm{ug} / \mathrm{I}$ & 5 & SW8021 & $\mathrm{MDH}$ & $465 \mathrm{~F}$ & 14 & Dec & 06 & DWR \\
\hline$m-x y l e n e$ and $p$-xylene & 4054 & $\mathrm{ug} / \mathrm{L}$ & 0.7 & SW8021 & MDH & $465 \mathrm{~F}$ & 14 & Dec & 06 & DWR \\
\hline o-xylene & 322.4 & $\mathrm{ug} / \mathrm{L}$ & 0.3 & SW8021 & MDH & $465 \mathrm{~F}$ & 14 & Dec & 06 & DWR \\
\hline
\end{tabular}

$R L=$ Reporting Limit 
JARDA SOLC

ENERGY \& ENVIRONMENTAL RESEARCH CTR

UNIVERSITY OF NORTH DAKOTA

GRAND FORKS ND 58203

\section{Sample Description: REC-1}

W5639
Page: 2 of 2

Report Date: 15 Dec 06

Lab Number: 06-A54139

work Order \#:82-2656

Account \#: 007033

Sample Matrix: GROUNDWATER

Date Sampled: 6 Dec 06 12:00

Date Received: 8 Dec 06

PO \#: CSI WATFORD

Temp at Receipt: $6.0 \mathrm{C}$

\begin{tabular}{|c|c|c|c|c|c|c|c|c|c|}
\hline \multirow[b]{2}{*}{ Cumene } & \multicolumn{2}{|c|}{$\begin{array}{l}\text { As Received } \\
\text { Result }\end{array}$} & \multirow{2}{*}{$\begin{array}{l}\begin{array}{l}\text { Method } \\
\text { RL }\end{array} \\
0.7\end{array}$} & \multicolumn{3}{|c|}{$\begin{array}{l}\text { Method } \\
\text { Reference }\end{array}$} & \multicolumn{2}{|c|}{$\begin{array}{l}\text { Date } \\
\text { Analyzed }\end{array}$} & \multirow{2}{*}{$\begin{array}{l}\text { Analyst } \\
\text { DWR }\end{array}$} \\
\hline & $<700 \#$ & $\mathrm{ug} / \mathrm{L}$ & & SW8021 & $\mathrm{MDH}$ & $465 \mathrm{~F}$ & 14 & Dec 06 & \\
\hline $1,1,1,2$-Tetrachloroethane & $<500 \#$ & $\mathrm{ug} / \mathrm{L}$ & 0.5 & SW8021 & $\mathrm{MDH}$ & $465 \mathrm{~F}$ & 14 & Dec 06 & DWR \\
\hline 1.1-Dichloropropene & $<500 \#$ & $\mathrm{ug} / \mathrm{L}$ & 0.5 & SW8021 & $\mathrm{MDH}$ & $465 \mathrm{~F}$ & 14 & Dec 06 & DWR \\
\hline Dichlorofluoromethane & $<500 \#$ & $\mathrm{ug} / \mathrm{L}$ & 0.5 & SW8021 & MDH & $465 \mathrm{~F}$ & 14 & Dec 06 & DWR \\
\hline Trichlorotrifluoroethane & $<900 \#$ & $\mathrm{ug} / \mathrm{I}$ & 0.9 & SW8021 $\mathrm{A}$ & $\mathrm{MDH}$ & $465 \mathrm{~F}$ & 14 & Dec 06 & DWR \\
\hline Ethyl Ether & $<800 \#$ & $\mathrm{ug} / \mathrm{L}$ & 0.8 & SW8021 1 & MDH & $465 \mathrm{~F}$ & 14 & Dec 06 & DWR \\
\hline Acetone & $<10000 \#$ & $\mathrm{ug} / \mathrm{L}$ & 10 & SW8021 & $\mathrm{MDH}$ & $465 \mathrm{~F}$ & 14 & Dec 06 & DWR \\
\hline Dibromomethane & $<700 \#$ & $\mathrm{ug} / \mathrm{L}$ & 0.7 & Sw8021 & MDH & $465 \mathrm{~F}$ & 1.4 & Dec 06 & DWR \\
\hline 2.2-Dichloropropane & $<1000 \#$ & $\mathrm{ug} / \mathrm{L}$ & 2 & SW8021 & MDH & $465 \mathrm{~F}$ & 14 & Dec 06 & DWR \\
\hline Bromochloromethane & $<600 \#$ & $\mathrm{ug} / \mathrm{L}$ & 0.6 & SWBO2I & $\mathrm{MDH}$ & $465 \mathrm{~F}$ & 14 & Dec 06 & DWR \\
\hline Methyl tert-butyl Ether & $<600 \#$ & $\mathrm{ug} / \mathrm{L}$ & 0.6 & SW8021 & $\mathrm{MDH}$ & $465 \mathrm{~F}$ & 14 & Dec 06 & DWR \\
\hline Styrene & $<500 \#$ & $\mathrm{ug} / \mathrm{L}$ & 0.5 & SWB021 & $\mathrm{MDH}$ & $465 \mathrm{~F}$ & 14 & Dec 06 & DWR \\
\hline n-Propylbenzene & $<800 \#$ & $\mathrm{ug} / \mathrm{L}$ & 0.8 & SW8021 & $\mathrm{MDH}$ & $465 \mathrm{~F}$ & 14 & Dec 06 & DWR \\
\hline Bromobenzene & $<500 \#$ & $\mathrm{ug} / \mathrm{L}$ & 0.5 & SW8021 & MDH & $465 \mathrm{~F}$ & 14 & Dec 06 & DWR \\
\hline 2-Chlorotoluene & $<800 \#$ & $\mathrm{ug} / \mathrm{L}$ & 0.8 & SW8021 & MDH & $465 \mathrm{~F}$ & 14 & Dec 06 & DWR \\
\hline 1,3,5-Trimethylbenzene & $<900 \#$ & $\mathrm{ug} / \mathrm{L}$ & 0.9 & SW8021 & $\mathrm{MDH}$ & $465 \mathrm{~F}$ & 14 & Dec 06 & DWR \\
\hline 4-Chlorotoluene & $<600 \#$ & $\mathrm{ug} / \mathrm{L}$ & 0.6 & SW8021 & $\mathrm{MDH}$ & $465 \mathrm{~F}$ & 24 & Dec 06 & DWR \\
\hline t-Butylbenzene & $<600 \#$ & $\mathrm{ug} / \mathrm{L}$ & 0.6 & SW8 021 & $\mathrm{MDH}$ & $465 \mathrm{~F}$ & 14 & Dec 06 & DWR \\
\hline $1,2,4$-Trimethylbenzene & 1899 & $\mathrm{ug} / \mathrm{L}$ & 0.7 & SW802I & $\mathrm{MDH}$ & $465 \mathrm{~F}$ & 14 & Dec 06 & DWR \\
\hline sec-Butylbenzene & $<700 \#$ & $\mathrm{ug} / \mathrm{L}$ & 0.7 & SW8021 & $\mathrm{MDH}$ & $465 \mathrm{~F}$ & 14 & Dec 06 & DWR \\
\hline p-Isopropyltoluene & $<700 \#$ & $\mathrm{ug} / \mathrm{L}$ & 0.7 & SW8021 & $\mathrm{MDH}$ & $465 \mathrm{~F}$ & 14 & Dec 06 & DWR \\
\hline I-Butylbenzene & $<700 \#$ & $\mathrm{ug} / \mathrm{L}$ & 0.7 & SW8021 & MDH & $465 \mathrm{~F}$ & 14 & Dec 06 & DWR \\
\hline 1,2-Dibromo-3-chloropropane & $<1000 \#$ & $\mathrm{ug} / \mathrm{L}$ & 1 & SW8021 & MDH & $465 \mathrm{~F}$ & 14 & Dec 06 & DWR \\
\hline $1,2,4$-Trichlorobenzene & $<500 \#$ & $\mathrm{ug} / \mathrm{L}$ & 0.5 & SW8021 & MDH & $465 \mathrm{~F}$ & 14 & Dec 06 & DWR \\
\hline Hexachlorobutadiene & $<700 \#$ & $\operatorname{ug} / \mathrm{L}$ & 0.7 & Sw8021 & MDH & $465 \mathrm{~F}$ & 14 & Dec 06 & DWR \\
\hline Naphthalene & $<1000 \#$ & $\mathrm{ug} / \mathrm{L}$ & 1 & SW8021 & $\mathrm{MDH}$ & $465 \mathrm{~F}$ & 14 & Dec 06 & DWR \\
\hline 1,2,3-Trichlorobenzene & $<600 \#$ & $\mathrm{ug} / \mathrm{L}$ & 0.6 & Sw8021 & $\mathrm{MDH}$ & $465 \mathrm{~F}$ & 14 & Dec 06 & DWR \\
\hline GRO (TPH) & 65.63 & $\mathrm{mg} / \mathrm{L}$ & 0.200 & $8015 \mathrm{~B} / 0$ & DAI & & 11 & Dec 06 & RDC \\
\hline
\end{tabular}

GC VoC sample $\mathrm{pH}>2$

1-CHLORO-4-FLUOROBENZENE (SURROGATE) RECOVERY: $101 \%$

BTEX/GRO Sample $\mathrm{pH}>2$

BTEX SURROGATE RECOVERY : $99 \%$

GRO SURROGATE RECOVERY: $111 \%$

GRO(TPH) pattern is characteristic of gasoline.

$R L=$ Reporting Limit

Elevated "Less Than Result" $(a): @=$ Due to sample matrix $:$ = Due to sample quantity
$\#$ = Due to sample concentration

- Due to extract volume 
JARDA SOLC

ENERGY \& ENVIRONMENTAL RESEARCH CTR

UNIVERSITY OF NORTH DAKOTA

GRAND FORKS ND 58203

\section{Sample Description: CHERRY CREEK} W5 635
Page: 1 of 2

Report Date: 15 Dec 06

Lab Number: 06-A54135

Work Order \#:82-2656

Account \#: 007033

Sample Matrix: GROUNDWATER

Date Sampled: 6 Dec 06 11:45

Date Received: 8 Dec 06

PO \#: CSI WATFORD

Temp at Receipt: $6.0 \mathrm{C}$

\begin{tabular}{|c|c|c|c|c|c|c|c|c|c|c|}
\hline \multirow{2}{*}{ Chloroethane } & \multicolumn{2}{|c|}{$\begin{array}{l}\text { As Received } \\
\text { Result }\end{array}$} & \multirow{2}{*}{$\begin{array}{l}\text { Method } \\
\text { RL } \\
0.7\end{array}$} & \multicolumn{3}{|c|}{$\begin{array}{l}\text { Method } \\
\text { Reference }\end{array}$} & \multicolumn{3}{|c|}{$\begin{array}{l}\text { Date } \\
\text { Analyzed }\end{array}$} & \multirow{2}{*}{$\begin{array}{l}\text { Analyst } \\
\text { DWR }\end{array}$} \\
\hline & $<0.7$ & $\mathrm{ug} / \mathrm{L}$ & & SW8021 & MDH & $465 \mathrm{~F}$ & 13 & Dec & 06 & \\
\hline Chloromethane & $<1$ & $\mathrm{ug} / \mathrm{L}$ & 1 & SWB021 & MDH & $465 \mathrm{~F}$ & 13 & Dec & 06 & DWR \\
\hline Bromomethane & $<0.7$ & $u g / L$ & 0.7 & SW8021 & MDH & $465 \mathrm{~F}$ & 13 & Dec & 06 & DWR \\
\hline Dichlorodif luoromet hane & $<0.9$ & $\mathrm{ug} / \mathrm{L}$ & 0.9 & SW8021 & $\mathrm{MDH}$ & $465 \mathrm{~F}$ & 13 & Dec & 06 & DWR \\
\hline Vinyl Chloride & $<0.5$ & $\mathrm{ug} / \mathrm{L}$ & 0.5 & SW8021 & $\mathrm{MDH}$ & $465 \mathrm{~F}$ & 13 & Dec & 06 & DWR \\
\hline Methylene Chloride & $<0.7$ & $\mathrm{ug} / \mathrm{L}$ & 0.7 & SW8021 & MDH & $465 \mathrm{~F}$ & 13 & Dec & 06 & DWR \\
\hline Trichlorofluoromethane & $<0.9$ & $\mathrm{ug} / \mathrm{L}$ & 0.9 & SW8021 & $\mathrm{MDH}$ & $465 \mathrm{~F}$ & 13 & Dec & 06 & DWR \\
\hline 1.1-Dichloroethene & $<0.5$ & $\mathrm{ug} / \mathrm{L}$ & 0.5 & SW8021 & $\mathrm{MDH}$ & $465 F$ & 13 & Dec & 06 & DWR \\
\hline 1,1-Dichloroethane & $<0.8$ & $\mathrm{ug} / \mathrm{L}$ & 0.8 & SW8021 & $\mathrm{MDH}$ & $465 F$ & 13 & Dec & 06 & DWR \\
\hline trans-1.2-Dichloroethene & $<0.5$ & $\mathrm{ug} / \mathrm{L}$ & 0.5 & SW8021 & MDH & $465 \mathrm{~F}$ & 13 & Dec & 06 & DWR \\
\hline Chloroform & $<0.8$ & $\mathrm{ug} / \mathrm{L}$ & 0.8 & SW8021 & $\mathrm{MDH}$ & $465 \mathrm{~F}$ & 13 & Dec & 06 & DWR \\
\hline 1.2-Dichloroethane & $<0.5$ & $\mathrm{ug} / \mathrm{L}$ & 0.5 & SW8021 & MDH & $465 \mathrm{~F}$ & 13 & Dec & 06 & DWR \\
\hline 1.1.1-Trichloroethane & $<0.9$ & $\mathrm{ug} / \mathrm{L}$ & 0.9 & SW8021 & $\mathrm{MDH}$ & $465 \mathrm{~F}$ & 13 & Dec & 06 & DWR \\
\hline Carbon Tetrachloride & $<0.8$ & $\mathrm{ug} / \mathrm{L}$ & 0.8 & SW8021 & $\mathrm{MDH}$ & $465 \mathrm{~F}$ & 13 & Dec & 06 & DWR \\
\hline Bromodichloromethane & $<0.5$ & $\mathrm{ug} / \mathrm{L}$ & 0.5 & SW8021 & $\mathrm{MDH}$ & $465 \mathrm{~F}$ & 13 & Dec & 06 & DWR \\
\hline 1.2-Dichloropropane & $<0.7$ & $\mathrm{ug} / \mathrm{L}$ & 0.7 & SW8021 & $\mathrm{MDH}$ & $465 \mathrm{~F}$ & 13 & Dec & 06 & DWR \\
\hline trans-1,3-Dichloropropene & $<0.5$ & $\mathrm{ug} / \mathrm{I}$ & 0.5 & SW8021 & $\mathrm{MDH}$ & $465 \mathrm{~F}$ & 13 & Dec & 06 & DWR \\
\hline Trichloroethene & $<0.5$ & $u g / L$ & 0.5 & SW802I & $\mathrm{MDH}$ & $465 \mathrm{~F}$ & 13 & Dec & 06 & DWR \\
\hline Chlorodibromomethane & $<0.5$ & $\mathrm{ug} / \mathrm{L}$ & 0.5 & SW8021 & $\mathrm{MDH}$ & $465 F$ & 13 & Dec & 06 & DWR \\
\hline $1,1,2-T r i c h l o r o e t h a n e$ & $<0.6$ & $\mathrm{ug} / \mathrm{L}$ & 0.6 & SWB 021 & MDH & $465 \mathrm{~F}$ & 13 & Dec & 06 & DWR \\
\hline cis-1,3-Dichloropropene & $<0.5$ & $\mathrm{ug} / \mathrm{L}$ & 0.5 & SW8021 & $\mathrm{MDH}$ & $465 \mathrm{~F}$ & 13 & Dec & 06 & DWR \\
\hline Bromoform & $<1$ & $\mathrm{ug} / \mathrm{L}$ & 1 & SW8021 & $\mathrm{MDH}$ & $465 \mathrm{~F}$ & 13 & Dec & 06 & DWR \\
\hline $1,1,2,2$-Tetrachloroethane & $<0.5$ & $\mathrm{ug} / \mathrm{L}$ & 0.5 & SW8021 & $\mathrm{MDH}$ & $465 \mathrm{~F}$ & 13 & Dec & 06 & DWR \\
\hline Tetrachloroethene & $<0.7$ & $\mathrm{ug} / \mathrm{L}$ & 0.7 & SW8021 & $\mathrm{MDH}$ & $465 \mathrm{~F}$ & 13 & Dec & 06 & DWR \\
\hline Chlorobenzene & $<0.6$ & $\mathrm{ug} / \mathrm{L}$ & 0.6 & SW8021 & MDH & $465 \mathrm{~F}$ & 13 & Dec & 06 & DWR \\
\hline Benzene & $<0.5$ & $\mathrm{ug} / \mathrm{L}$ & 0.5 & Sw8021 & $\mathrm{MDH}$ & $465 \mathrm{~F}$ & 13 & Dec & 06 & DWR \\
\hline Toluene & $<0.6$ & $\mathrm{ug} / \mathrm{L}$ & 0.6 & SW8021 & $\mathrm{MDH}$ & $465 \mathrm{~F}$ & 13 & Dec & 06 & DWR \\
\hline Ethyl Benzene & $<0.6$ & $\mathrm{ug} / \mathrm{L}$ & 0.6 & SW8021 & $\mathrm{MDH}$ & $465 \mathrm{~F}$ & 13 & Dec & 06 & DWR \\
\hline 1.2-Dichlorobenzene & $<0.5$ & $\mathrm{ug} / \mathrm{L}$ & 0.5 & SW8021 & $\mathrm{MDH}$ & $465 \mathrm{~F}$ & 13 & Dec & 06 & DWR \\
\hline 1.3-Dichlorobenzene & $<0.5$ & $\mathrm{ug} / \mathrm{L}$ & 0.5 & SWB 021 & MDH & $465 \mathrm{~F}$ & 13 & Dec & 06 & DWR \\
\hline 1.4-Dichlorobenzene & $<0.5$ & $\mathrm{ug} / \mathrm{L}$ & 0.5 & SWB 021 & $\mathrm{MDH}$ & $465 \mathrm{~F}$ & 13 & Dec & 06 & DWR \\
\hline cis-1,2-Dichloroethene & $<0.5$ & $\mathrm{ug} / \mathrm{L}$ & 0.5 & SW8021 & $\mathrm{MDH}$ & $465 \mathrm{~F}$ & 13 & Dec & 06 & DWR \\
\hline 1.3-Dichloropropane & $<0.6$ & $\mathrm{ug} / \mathrm{L}$ & 0.6 & SWB 021 & $\mathrm{MDH}$ & $465 \mathrm{~F}$ & 13 & Dec & 06 & DWR \\
\hline $1,2,3$-Trichloropropane & $<0.5$ & $\mathrm{ug} / \mathrm{L}$ & 0.5 & SW8021 & $\mathrm{MDH}$ & $465 \mathrm{~F}$ & 13 & Dec & 06 & DWR \\
\hline Allyl Chloride & $<0.7$ & $\mathrm{ug} / \mathrm{L}$ & 0.7 & SW8021 & $\mathrm{MDH}$ & $465 \mathrm{~F}$ & 13 & Dec & 06 & DWR \\
\hline 1,2-Dibromoethane & $<0.5$ & $\mathrm{ug} / \mathrm{L}$ & 0.5 & SW8021 & $\mathrm{MDH}$ & $465 \mathrm{~F}$ & 13 & Dec & 06 & DWR \\
\hline Methyl Ethyl Ketone & $<5$ & $\mathrm{ug} / \mathrm{L}$ & 5 & SW8021 & $\mathrm{MDH}$ & $465 \mathrm{~F}$ & 13 & Dec & 06 & DWR \\
\hline Methyl Isobutyl Ketone & $<1.4$ & $u g / L$ & 1.4 & SWB021 & $\mathrm{MDH}$ & $465 \mathrm{~F}$ & 13 & Dec & 06 & DWR \\
\hline Tetrahydrofuran & $<5$ & $\mathrm{ug} / \mathrm{L}$ & 5 & SWBO21 & $\mathrm{MDH}$ & $465 \mathrm{~F}$ & 13 & Dec & 06 & DWR \\
\hline$m-x y l e n e$ and $p-x y l e n e$ & $<0.7$ & $\mathrm{ug} / \mathrm{L}$ & 0.7 & SW8021 & $\mathrm{MDH}$ & $465 \mathrm{~F}$ & 13 & Dec & 06 & DWR \\
\hline o-xylene & $<0.3$ & $\mathrm{ug} / \mathrm{L}$ & 0.3 & SW802I & MDH & $465 \mathrm{~F}$ & 13 & Dec & 06 & DWR \\
\hline
\end{tabular}

RL. = Reporting l,imit 


\author{
JARDA SOLC \\ ENERGY \& ENVIRONMENTAL RESEARCH CTR \\ UNIVERSITY OF NORTH DAKOTA \\ GRAND FORKS ND 58203
}

\section{Sample Description: CHERRY CREEK W5635}

\author{
Page: 2 of 2 \\ Report Date: 15 Dec 06 \\ Lab Number: 06-A54135 \\ Work Order \#:82-2656 \\ Account \#: 007033 \\ Sample Matrix: GROUNDWATER \\ Date Sampled: 6 Dec 06 11:45 \\ Date Received: 8 Dec 06 \\ PO \# : CSI WATFORD \\ Temp at Receipt: $6.0 \mathrm{C}$
}

As Received

Result

Method

Method

Date

Reference

Analyst

Cumene

$<0.7 \quad \mathrm{ug} / \mathrm{L}$

$1,1,1,2$-Tetrachloroethane

$<0.5$

ug/L

$<0.5 \mathrm{ug} / \mathrm{L}$

$<0.5 \mathrm{ug} / \mathrm{L}$

$<0.9 \quad \mathrm{ug} / \mathrm{L}$

Trichlorotrifluoroethane

$u g / L$
$u g / L$

$<0.8$

13.6

$<0.7$

ug/L

$\mathrm{ug} / \mathrm{L}$

Dibromomethane

2,2-Dichloropropane

Bromochlorome thane

methyl tert-butyl Ether

$<1$

$<0.6$

$\mathrm{ug} / \mathrm{L}$

$\mathrm{ug} / \mathrm{L}$

$<0.6$

$<0.5$

$<0.8$

ug/L

n-Propylbenzene

Bromobenzene

2-Chlorotoluene

1,3,5-Trimethyibenzene

$<0.5$

$<0.8$

$<0.9$

$<0.6$

$<0.6$

$<0.7$

$<0.7$

$<0.7$

$<0.7$

$<1$

$<0.5$

$<0.7$

$<1$

$<0.6$

ug/L

$\mathrm{ug} / \mathrm{L}$

ug/L.

$\mathrm{ug} / \mathrm{L}$

$\mathrm{ug} / \mathrm{L}$

$\mathrm{ug} / \mathrm{L}$

$\mathrm{ug} / \mathrm{L}$

$u g / L$

$\mathrm{ug} / \mathrm{L}$

$\mathrm{ug} / \mathrm{I}$,

ug/L

$\mathrm{ug} / \mathrm{L}$

$\mathrm{ug} / \mathrm{L}$

$\mathrm{ug} / \mathrm{L}$

$\mathrm{ug} / \mathrm{L}$

$\mathrm{ug} / \mathrm{I}$

$\mathrm{mg} / \mathrm{L}$,

RI

\begin{tabular}{|c|c|c|}
\hline SWBO21 & $\mathrm{MDH}$ & $465 \mathrm{~F}$ \\
\hline SW8021 & $\mathrm{MDH}$ & $65 \mathrm{~F}$ \\
\hline WBO21 & $\mathrm{MDH}$ & $465 \mathrm{~F}$ \\
\hline SW8021 & $\mathrm{MDH}$ & $465 \mathrm{~F}$ \\
\hline SW8021 & $\mathrm{MDH}$ & $465 \mathrm{~F}$ \\
\hline SW8021 & $\mathrm{MDH}$ & $465 \mathrm{~F}$ \\
\hline SW8021 & MDH & $465 \mathrm{~F}$ \\
\hline SW8021 & $\mathrm{MDH}$ & $465 \mathrm{~F}$ \\
\hline SWBO21 & $\mathrm{MDH}$ & $465 \mathrm{~F}$ \\
\hline $3 W 8021$ & $\mathrm{MDH}$ & $465 \mathrm{~F}$ \\
\hline SWBO 21 & $\mathrm{MDH}$ & $465 \mathrm{~F}$ \\
\hline SW8021 & $\mathrm{MDH}$ & $465 \mathrm{~F}$ \\
\hline SWBO21 & $\mathrm{MDH}$ & $465 \mathrm{~F}$ \\
\hline SW802I & $\mathrm{MDH}$ & $465 \mathrm{~F}$ \\
\hline SW8021 & $\mathrm{MDH}$ & $465 \mathrm{~F}$ \\
\hline SWBO21 & $\mathrm{MDH}$ & $465 \mathrm{~F}$ \\
\hline SW8021 & $\mathrm{MDH}$ & $465 F$ \\
\hline SWB021 & $\mathrm{MDH}$ & $465 \mathrm{~F}$ \\
\hline SW8021 & $\mathrm{MDH}$ & $465 \mathrm{~F}$ \\
\hline SW8021 & $\mathrm{MDH}$ & $465 \mathrm{~F}$ \\
\hline SWB021 & $\mathrm{MDH}$ & $465 \mathrm{~F}$ \\
\hline SWE 021 & $\mathrm{MDH}$ & $465 F$ \\
\hline SW8021 & $\mathrm{MDH}$ & $465 \mathrm{~F}$ \\
\hline SW8021 & $\mathrm{MDH}$ & $465 \mathrm{~F}$ \\
\hline SW8021 & MDH & $465 \mathrm{~F}$ \\
\hline SW8021 & $\mathrm{MDH}$ & $465 \mathrm{~F}$ \\
\hline SW8021 & $\mathrm{MDH}$ & $465 \mathrm{~F}$ \\
\hline
\end{tabular}

13 Dec 06

13 Dec 06

13 Dec 06

13 Dec 06

13 Dec 06

13 Dec 06

13 Dec 06

13 DeC 06

13 Dec 06

13 Dec 06

13 Dec 06

13 Dec 06

13 Dec 06

13 DeC 06

13 DeC 06

13 Dec 06

13 Dec 06

13 Dec 06

13 Dec 06

13 Dec 06

13 Dec 06

13 Dec 06

13 Dec 06

13 Dec 06

13 Dec 06

13 Dec 06

13 Dec 06

$11 \mathrm{DeC} 06$

DWR
DWR
DWR
DWR
DWR
DWR
DWR
DWR
DWR
DWR
DWR
DWR
DWR
DWR
DWR
DWR
DWR
DWR
DWR
DWR
DWR
DWR
DWR
DWR
DWR
DWR
DWR
RDC

GC VOC Sample pH $<2$

1-CHLORO-4-FLUOROBENZENE (SURROGATE) RECOVERY: $103 \%$

BTEX/GRO Sample pH $<2$

BTEX SURROGATE RECOVERY : $84 \%$

GRO SURROGATE RECOVERY : $95 \%$ 
JARDA SOLC

ENERGY \& ENVIRONMENTAL RESEARCH CTR UNIVERSITY OF NORTH DAKOTA

GRAND FORKS ND 58203

\section{Sample Description: MUNICIPAL WELL} W5636
Page: 1 of 2

Report Date: 15 Dec 06

Lab Number: 06-A54136

Work Order \#:82-2656

Account \#: 007033

Sample Matrix: GROUNDWATER

Date Sampled: 6 Dec 06 15:35

Date Received: 8 Dec 06

PO \# : CSI WATFORD

Temp at Receipt: $6.0 \mathrm{C}$

As Received

Result

Method Method Date

RL Reference Analyzed

\section{Chloroethane}

chloromethane

Bromomethane

Dichlorodifluoromethane

Vinyl Chloride

Methylene Chloride

Trichlorofluoromethane

1.1-Dichloroethere

1,1-Dichloroethane

$<0.7$

$\mathrm{ug} / \mathrm{L}$

$<1$

$<0.7$
$<0.9$

ug $/ 1$.

$<0.5$

$<0.7$

$\mathrm{ug} / \mathrm{L}$

ug/L

$u g / L$

$u g / L$

$<0.9$

$<0.5$

$u g / L$

ug/L

$<0.8$

$u g / L$
trans-1,2-Dichloroethene

Chloroform

1,2-Dichloroethane

1,1,1-Trichloroethane

Carbon Tetrachloride

Bromodichloromethane

1,2-Dichloropropane

trans-1,3-Dichloropropene

Trichloroethene

Chlorodibromomethane

1.1.2-Trichloroe thane

cis-1,3-Dichloropropene

Bromoform

1,1,2,2-Tetrachloroethane

$<0.5$

$<0.8$

$<0.5$

$<0.9$

$<0.8$

$<0.5$

$<0.7$

$<0.5$

$<0.5$

$<0.5$

$<0.6$

$<0.5$

$<1$

$<0.5$

$<0.7$

$<0.7$
$<0.6$

$<0.5$

$<0.5$
$<0.6$

$<0.6$

$<0.5$

$<0.5$

$<0.5$

$<0.5$

$<0.6$

$<0.5$

$<0.7$

$<0.5$

$<5$

$<1.4$

$<5$

$<0.7$

$<0.3$

ug/L

ug/L

ug/L

ug/L

$\mathrm{ug} / \mathrm{L}$

ug/L

$\mathrm{ug} / \mathrm{L}$

$\mathrm{ug} / \mathrm{L}$

$\mathrm{ug} / \mathrm{L}$

$\mathrm{ug} / \mathrm{L}$

$\mathrm{ug} / \mathrm{L}$

$\mathrm{Lg} / \mathrm{L}$

ug $/ \mathrm{L}$.

$\mathrm{ug} / \mathrm{L}$

ug/L.

$\mathrm{ug} / \mathrm{L}$

$\mathrm{ug} / \mathrm{L}$

ug/L.

ug/L.

$\mathrm{ug} / \mathrm{L}$

$\mathrm{ug} / \mathrm{L}$

ug/L

ug/L,

ug/I.

$\mathrm{ug} / \mathrm{L}$

$\mathrm{ug} / \mathrm{L}$

ug/L.

$\mathrm{ug} / \mathrm{L}$

Methyl Ethyl Ketone

Methyl Isobutyl
Tetrahydrofuran

m-xylene and $p$-xylene

o-xylene $\mathrm{ug} / \mathrm{L}$

ug/I

$u g / L$

$u g / L$
SW8021 MDH 465F

SW8021 MDH 465F

SW8021 MDH $465 \mathrm{~F}$

SW8021 MDH 465F

SW8021 MDH 465F

SW8021 MDH 465F

SW8021 MDH 465F

SW802I MDH 465F

SWB021 MDH 465F

SW8021 MDH 465F

SW8021 MDH $465 \mathrm{~F}$

SW8021 MDH 465F

SW8021 MDH 465F

SW8021 MDH 465F

SW8021 MDH 465F

SW8021 MDH $465 \mathrm{~F}$

SW8021 MDH $465 \mathrm{~F}$

SWB $021 \mathrm{MDH} 465 \mathrm{~F}$

SW8021 MDH $465 \mathrm{~F}$

SW8021 MDH $465 \mathrm{~F}$

SW8021 MDH $465 \mathrm{~F}$

SW8021 MDH 465F

SWB021 MDH 465F

SW8021 MDH $465 \mathrm{~F}$

SW8021 MDH 465F

SW8021 MDH 465F

SW8021 MDH $465 \mathrm{~F}$

SW8021 MDH 465F

SW8021 MDH 465F

SW8021 MDH $465 \mathrm{~F}$

SW8021 MDH 465F

SW8021 MDH $465 \mathrm{~F}$

SW8021 MDH $465 \mathrm{~F}$

SW8021 MDH 465F

SW8021 MDH $465 \mathrm{~F}$

SW8021 MDH 465F

SW8021 MDH $465 \mathrm{~F}$

SW8021 MDH 465F

SW8021 MDH 465F

SW8021 MDH 465F
SW8021 MDH 465F

\begin{tabular}{|c|c|c|c|}
\hline 13 & Dec & 06 & DWR \\
\hline 13 & Dec & 06 & DWR \\
\hline 13 & Dec & 06 & DWR \\
\hline 13 & Dec & 06 & DWR \\
\hline 13 & Dec & 06 & DWR \\
\hline 13 & Dec & 06 & DWR \\
\hline 13 & Dec & 06 & DWR \\
\hline 13 & DeC & 06 & DWR \\
\hline 13 & Dec & 06 & DWR \\
\hline 13 & DeC & 06 & DWR \\
\hline 13 & Dec & 06 & DWR \\
\hline 13 & Dec & 06 & DWR \\
\hline 13 & Dec & 06 & DWR \\
\hline 13 & Dec & 06 & DWR \\
\hline 13 & Dec & 06 & DWR \\
\hline 13 & Dec & 06 & DWR \\
\hline 13 & Dec & 06 & DWR \\
\hline 13 & Dec & 06 & DWR \\
\hline 13 & Dec & 06 & DWR \\
\hline 13 & Dec & 06 & DWR \\
\hline 13 & Dec & 06 & DWR \\
\hline 13 & Dec & 06 & DWR \\
\hline 13 & Dec & 06 & DWR \\
\hline 13 & Dec & 06 & DWR \\
\hline 13 & Dec & 06 & DWR \\
\hline 13 & Dec & 06 & DWR \\
\hline 13 & Dec & 06 & DWR \\
\hline 13 & DeC & 06 & DWR \\
\hline 13 & Dec & 06 & DWR \\
\hline 13 & Dec & 06 & DWR \\
\hline 13 & Dec & 06 & DWR \\
\hline 13 & Dec & 06 & DWR \\
\hline 13 & Dec & 06 & DWR \\
\hline 13 & Dec & 06 & DWR \\
\hline 13 & Dec & 06 & DWR \\
\hline 13 & Dec & 06 & DWR \\
\hline 13 & Dec & 06 & DWR \\
\hline 13 & Dec & 06 & DWR \\
\hline 13 & Dec & 06 & DWR \\
\hline 13 & Dec & 06 & DWR \\
\hline 13 & Dec & 06 & DWR \\
\hline
\end{tabular}


JARDA SOLC

ENERGY \& ENVIRONMENTAL RESEARCH CTR

UNIVERSITY OF NORTH DAKOTA

GRAND FORKS ND 58203

\section{Sample Description: MUNICIPAL WELL W5636}

\begin{tabular}{|c|c|c|c|c|c|c|c|c|c|}
\hline \multirow[b]{2}{*}{ Cumene } & \multicolumn{2}{|c|}{$\begin{array}{l}\text { As Received } \\
\text { Result }\end{array}$} & \multirow{2}{*}{$\begin{array}{l}\begin{array}{l}\text { Method } \\
\text { RL. }\end{array} \\
0.7\end{array}$} & \multicolumn{3}{|c|}{$\begin{array}{l}\text { Method } \\
\text { Reference }\end{array}$} & \multicolumn{2}{|c|}{$\begin{array}{l}\text { Date } \\
\text { Analyzed }\end{array}$} & \multirow{2}{*}{$\begin{array}{l}\text { Analyst } \\
\text { DWR }\end{array}$} \\
\hline & $<0.7$ & $\mathrm{ug} / \mathrm{L}$ & & SW8021 & $\mathrm{MDH}$ & $465 \mathrm{~F}$ & 13 & Dec 06 & \\
\hline $1,1,1,2$-Tetrachloroethane & $<0.5$ & $\mathrm{ug} / \mathrm{L}$ & 0.5 & Sw8021 & MDH & $465 \mathrm{~F}$ & 13 & Dec 06 & DWR \\
\hline 1,1-Dichloropropene & $<0.5$ & $\mathrm{ug} / \mathrm{L}$ & 0.5 & SW8021 & $\mathrm{MDH}$ & $465 \mathrm{~F}$ & 13 & Dec 06 & DWR \\
\hline Dichlorofluoromethane & $<0.5$ & $\mathrm{ug} / \mathrm{L}$ & 0.5 & SW8021 & $\mathrm{MDH}$ & $465 \mathrm{~F}$ & 13 & Dec 06 & DWR \\
\hline Trichlorotrifluoroethane & $<0.9$ & $\mathrm{ug} / \mathrm{L}$ & 0.9 & SW8021 & $\mathrm{MDH}$ & $465 \mathrm{~F}$ & 13 & Dec 06 & DWR \\
\hline Ethyl Ether & $<0.8$ & $\mathrm{ug} / \mathrm{L}$ & 0.8 & SW8021 & $\mathrm{MDH}$ & $465 \mathrm{~F}$ & 13 & Dec 06 & DWR \\
\hline Acetone & $<10$ & $\mathrm{ug} / \mathrm{L}$ & 10 & $5 W 8021$ & $\mathrm{MDH}$ & $465 \mathrm{~F}$ & 13 & Dec 06 & DWR \\
\hline Dibromomethane & $<0.7$ & $\mathrm{ug} / \mathrm{L}$ & 0.7 & SW8021 & $\mathrm{MDH}$ & $465 \mathrm{~F}$ & 13 & Dec 06 & DWR \\
\hline 2,2-Dichloropropane & $<1$ & $\mathrm{ug} / \mathrm{L}$ & 1 & SW8021 & $\mathrm{MDH}$ & $465 \mathrm{~F}$ & 13 & Dec 06 & DWR \\
\hline Bromochloromethane & $<0.6$ & $\mathrm{ug} / \mathrm{L}$ & 0.6 & SW8021 & $\mathrm{MDH}$ & $465 \mathrm{~F}$ & 13 & Dec 06 & DWR \\
\hline Methyl tert-butyl Ether & $<0.6$ & $\mathrm{ug} / \mathrm{L}$ & 0.6 & SW8021 & MDH & $465 \mathrm{~F}$ & 13 & Dec 06 & DWR \\
\hline styrene & $<0.5$ & $\mathrm{ug} / \mathrm{L}$ & 0.5 & SW8021 & $\mathrm{MDH}$ & $465 \mathrm{~F}$ & 13 & Dec 06 & DWR \\
\hline n-propylbenzene & $<0.8$ & $\mathrm{ug} / \mathrm{L}$ & 0.8 & SWB 021 & MDH & $465 \mathrm{~F}$ & 13 & Dec 06 & DWR \\
\hline Bromobenzene & $<0.5$ & $\mathrm{ug} / \mathrm{L}$ & 0.5 & SW8021 & $\mathrm{MDH}$ & $465 \mathrm{~F}$ & 13 & Dec 06 & DWR \\
\hline 2-Chlorotoluene & $<0.8$ & $\mathrm{ug} / \mathrm{L}$ & 0.8 & SW8021 & $\mathrm{MDH}$ & $465 \mathrm{~F}$ & 13 & Dec 06 & DWR \\
\hline 1,3,5-Trimethylbenzene & $<0.9$ & $\mathrm{ug} / \mathrm{L}$ & 0.9 & Sw8 021 & $\mathrm{MDH}$ & $465 \mathrm{~F}$ & 13 & Dec 06 & DWR \\
\hline 4-Chlorotoluene & $<0.6$ & $\mathrm{ug} / \mathrm{L}$ & 0.6 & SW8021 & MDH & $465 \mathrm{~F}$ & 13 & Dec 06 & DWR \\
\hline$t$-Butylbenzene & $<0.6$ & $\mathrm{ug} / \mathrm{L}$ & 0.6 & SW8021 & MDH & $465 \mathrm{~F}$ & 13 & Dec 06 & DWR \\
\hline 1.2,4-Trimethylbenzene & $<0.7$ & $\mathrm{ug} / \mathrm{L}$ & 0.7 & SW8021 & $\mathrm{MDH}$ & $465 \mathrm{~F}$ & 13 & Dec 06 & DWR \\
\hline sec-Butylbenzene & $<0.7$ & $\mathrm{ug} / \mathrm{L}$ & 0.7 & SW8021 & $\mathrm{MDH}$ & $465 \mathrm{~F}$ & 13 & Dec 06 & DWR \\
\hline p-Isopropyltoluene & $<0.7$ & $\mathrm{ug} / \mathrm{L}$ & 0.7 & SW8021 & $\mathrm{MDH}$ & $465 \mathrm{~F}$ & 13 & Dec 06 & DWR \\
\hline n-Butylbenzene & $<0.7$ & $\mathrm{ug} / \mathrm{L}$ & 0.7 & Sw8021. & $\mathrm{MDH}$ & $465 \mathrm{~F}$ & 1.3 & Dec 06 & DWR \\
\hline 1,2-Dibromo-3-chloropropane & $<1$ & $\mathrm{ug} / \mathrm{L}$ & 1 & SW8021 & $\mathrm{MDH}$ & $465 \mathrm{~F}$ & 13 & Dec 06 & DWR \\
\hline 1,2,4-Trichlorobenzene & $<0.5$ & $\mathrm{ug} / \mathrm{L}$ & 0.5 & SW8021 & $\mathrm{MDH}$ & $465 \mathrm{~F}$ & 13 & Dec 06 & DWR \\
\hline Hexachlorobutadiene & $<0.7$ & $\mathrm{ug} / \mathrm{L}$ & 0.7 & SW8021 & $\mathrm{MDH}$ & $465 \mathrm{~F}$ & 13 & Dec 06 & DWR \\
\hline Naphthalene & $<1$ & $\mathrm{ug} / \mathrm{L}$ & 1 & SW8021 & $\mathrm{MDH}$ & $465 \mathrm{~F}$ & 13 & Dec 06 & DWR \\
\hline 1,2,3-Trichlorobenzene & $<0.6$ & $\mathrm{ug} / \mathrm{L}$ & 0.6 & SW8021 & $\mathrm{MDH}$ & $465 \mathrm{~F}$ & 13 & Dec 06 & DWR \\
\hline GRO (TPH) & $<0.2$ & $\mathrm{mg} / \mathrm{L}$ & 0.2 & $8015 B / C$ & DA1 & & 8 & Dec 06 & RDC \\
\hline
\end{tabular}

GC VOC sample $\mathrm{pH}<2$

1-CHLORO-4-FLUOROBENZENE (SURROGATE) RECOVERY： $99 \frac{\circ}{\frac{\circ}{a}}$

BTEX/GRO Sample $\mathrm{pH}<2$

BTEX SURROGATE RECOVERY: $104 \%$

GRO SURROGATE RECOVERY : $96 \div$
Page: 2 of 2

Report Date: 15 Dec 06

Lab Number: $06-$ A5 4136

Work Order \#:82-2656

Account \#: 007033

Sample Matrix: GROUNDWATER

Date Sampled: 6 Dec 06 15:35

Date Received: 8 Dec 06

PO \#: CSI WATFORD

Temp at Receipt: $6.0 \mathrm{C}$ 
JARDA SOLC

ENERGY \& ENVIRONMENTAL RESEARCH CTR

UNIVERSITY OF NORTH DAKOTA

GRAND FORKS ND 58203
Page: 1 of 2

Report Date: 15 Dec 06

Lab Number: 06-A54141

work Order \#:82-2656

Account \#: 007033

Sample Matrix: GROUNDWATER

Date Sampled: 6 Dec 06

Date Received: 8 Dec 06

PO \#: CSI WATFORD

\author{
Sample Description: FIELD BLANK \\ W5641
}

Temp at Receipt: $6.0 \mathrm{C}$

\begin{tabular}{|c|c|c|c|c|c|c|c|c|c|}
\hline \multirow[b]{2}{*}{ Chloroethane } & \multicolumn{2}{|c|}{$\begin{array}{l}\text { As Received } \\
\text { Result }\end{array}$} & \multirow{2}{*}{$\begin{array}{l}\text { Method } \\
\text { RL } \\
0.7\end{array}$} & \multicolumn{3}{|c|}{$\begin{array}{l}\text { Method } \\
\text { Reference }\end{array}$} & \multicolumn{2}{|c|}{$\begin{array}{l}\text { Date } \\
\text { Analyzed }\end{array}$} & \multirow{2}{*}{$\begin{array}{l}\text { Analyst } \\
\text { DWR }\end{array}$} \\
\hline & $<0.7$ & $\mathrm{ug} / \mathrm{L}$ & & SW8021 & $\mathrm{MDH}$ & $465 \mathrm{~F}$ & 14 & Dec 06 & \\
\hline Chlorome thane & $<1$ & $\mathrm{ug} / \mathrm{L}$ & 1 & SW8021 & $\mathrm{MDH}$ & $465 \mathrm{~F}$ & 14 & Dec 06 & DWR \\
\hline Bromomethane & $<0.7$ & $\mathrm{ug} / \mathrm{L}$ & 0.7 & Sw8021 & MDH & $465 \mathrm{~F}$ & 14 & Dec 06 & DWR \\
\hline Dichlorodifluoromethane & $<0.9$ & $\mathrm{ug} / \mathrm{L}$ & 0.9 & SW8021 & $\mathrm{MDH}$ & $465 \mathrm{~F}$ & 24 & Dec 06 & DWR \\
\hline vinyl Chloride & $<0.5$ & $\mathrm{ug} / \mathrm{L}$ & 0.5 & SWB021 & MDH & $465 F$ & 14 & Dec 06 & DWR \\
\hline Methylene Chloride & $<0.7$ & $\mathrm{ug} / \mathrm{L}$ & 0.7 & SW8021 & MDH & $465 \mathrm{~F}$ & 14 & Dec 06 & DWR \\
\hline Trichlorofluoromethane & $<0.9$ & $\mathrm{ug} / \mathrm{L}$ & 0.9 & SWBO2I & MDH & $465 \mathrm{~F}$ & 14 & Dec 06 & DWR \\
\hline 1.1-Dichloroethene & $<0.5$ & $\mathrm{ug} / \mathrm{L}$ & 0.5 & SW8021 & $\mathrm{MDH}$ & $465 \mathrm{~F}$ & 14 & Dec 06 & DWR \\
\hline 1.1-Dichloroethane & $<0.8$ & $\mathrm{ug} / \mathrm{L}$ & 0.8 & SW8021 & MDH & $465 \mathrm{~F}$ & 14 & Dec 06 & DWR \\
\hline trans-1,2-Dichloroethene & $<0.5$ & $\mathrm{ug} / \mathrm{L}$ & 0.5 & SWB 021 & $\mathrm{MDH}$ & $465 \mathrm{~F}$ & 14 & Dec 06 & DWR \\
\hline chloroform & $<0.8$ & ug/L & 0.8 & SW8021 & $\mathrm{MDH}$ & $465 \mathrm{~F}$ & 14 & Dec 06 & DWR \\
\hline 1,2-Dichloroethane & $<0.5$ & $\mathrm{ug} / \mathrm{L}$ & 0.5 & SW8021 & $\mathrm{MDH}$ & $465 \mathrm{~F}$ & 14 & Dec 06 & DWR \\
\hline 1.1.1-Trichloroethane & $<0.9$ & $\mathrm{ug} / \mathrm{L}$ & 0.9 & SW8021 & $\mathrm{MDH}$ & $465 \mathrm{~F}$ & 14 & Dec 06 & DWR \\
\hline Carbon Tetrachloride & $<0.8$ & $\mathrm{ug} / \mathrm{L}$ & 0.8 & SW8021 & MDH & $465 \mathrm{~F}$ & 14 & Dec 06 & DWR \\
\hline Bromodichlorome thane & $<0.5$ & $\mathrm{ug} / \mathrm{L}$ & 0.5 & SW8021 & MDH & $465 \mathrm{~F}$ & 14 & Dec 06 & DWR \\
\hline 1,2-Dichloropropane & $<0.7$ & $\mathrm{ug} / \mathrm{L}$ & 0.7 & SW8021 & MDH & $465 \mathrm{~F}$ & 14 & Dec 06 & DWR \\
\hline trans-1,3-Dichloropropene & $<0.5$ & $\mathrm{ug} / \mathrm{L}$ & 0.5 & SWB 021 & $\mathrm{MDH}$ & $465 \mathrm{~F}$ & 14 & Dec 06 & DWR \\
\hline Trichloroethene & $<0.5$ & $\mathrm{ug} / \mathrm{L}$ & 0.5 & SW8 021 & MDH & $465 \mathrm{~F}$ & 14 & Dec 06 & DWR \\
\hline Chlorodibromomethane & $<0.5$ & $\mathrm{ug} / \mathrm{L}$ & 0.5 & SW8021 & $\mathrm{MDH}$ & $465 \mathrm{~F}$ & 14 & Dec 06 & DWR \\
\hline 1.1.2-Trichloroethane & $<0.6$ & $\mathrm{ug} / \mathrm{L}$ & 0.6 & SW8021 & $\mathrm{MDH}$ & $465 \mathrm{~F}$ & 14 & Dec 06 & DWR \\
\hline cis-1,3-Dichloropropene & $<0.5$ & $\mathrm{ug} / \mathrm{L}$ & 0.5 & SW8021 & MDH & $465 \mathrm{~F}$ & 14 & Dec 06 & DWR \\
\hline Bromoform & $<1$ & $\mathrm{ug} / \mathrm{L}$ & 1 & SW8021 & $\mathrm{MDH}$ & $465 \mathrm{~F}$ & 14 & Dec 06 & DWR \\
\hline $1,1,2,2$-Tetrachloroethane & $<0.5$ & $\mathrm{ug} / \mathrm{L}$ & 0.5 & SW8 021 & $\mathrm{MDH}$ & $465 \mathrm{~F}$ & 14 & Dec 06 & DWR \\
\hline Tetrachloroethene & $<0.7$ & $\mathrm{ug} / \mathrm{L}$ & 0.7 & SW8021 & MDH & $465 \mathrm{~F}$ & 14 & Dec 06 & DWR \\
\hline Chlorobenzene & $<0.6$ & $\mathrm{ug} / \mathrm{I}$ & 0.6 & SW8021 & $\mathrm{MDH}$ & $465 \mathrm{~F}$ & 14 & Dec 06 & DWR \\
\hline Benzene & $<0.5$ & $\mathrm{ug} / \mathrm{L}$ & 0.5 & SW8021 & $\mathrm{MDH}$ & $465 \mathrm{~F}$ & 1.4 & Dec 06 & DWR \\
\hline Toluene & $<0.6$ & $\mathrm{ug} / \mathrm{L}$ & 0.6 & SW8021 & $\mathrm{MDH}$ & $465 \mathrm{~F}$ & 14 & Dec 06 & DWR \\
\hline Ethyl Benzene & $<0.6$ & $\mathrm{ug} / \mathrm{L}$ & 0.6 & SW8021 & MDH & $465 \mathrm{~F}$ & 14 & Dec 06 & DWR \\
\hline 1,2-Dichlorobenzene & $<0.5$ & $\mathrm{ug} / \mathrm{L}$ & 0.5 & SW8021 & $\mathrm{MDH}$ & $465 \mathrm{~F}$ & 14 & Dec 06 & DWR \\
\hline 1,3-Dichlorobenzene & $<0.5$ & $\mathrm{ug} / \mathrm{L}$ & 0.5 & SW8021 & MDH & $465 \mathrm{~F}$ & 14 & Dec 06 & DWR \\
\hline 1,4-Dichlorobenzene & $<0.5$ & $\mathrm{ug} / \mathrm{L}$ & 0.5 & SW8021 & MDH & $465 \mathrm{~F}$ & 14 & Dec 06 & DWR \\
\hline cis-1,2-Dichloroethene & $<0.5$ & $\mathrm{ug} / \mathrm{L}$ & 0.5 & SW802I & $\mathrm{MDH}$ & $465 \mathrm{~F}$ & 14 & Dec 06 & DWR \\
\hline 1,3-Dichloropropane & $<0.6$ & $\mathrm{ug} / \mathrm{L}$ & 0.6 & SW8021 & MDH & $465 \mathrm{~F}$ & 14 & Dec 06 & DWR \\
\hline 1,2,3-Trichloropropane & $<0.5$ & $\mathrm{ug} / \mathrm{L}$ & 0.5 & SW8021 & MDH & $465 \mathrm{~F}$ & 14 & Dec 06 & DWR \\
\hline Allyl Chloride & $<0.7$ & $\mathrm{ug} / \mathrm{L}$ & 0.7 & SW8021 & MDH & $465 \mathrm{~F}$ & 14 & Dec 06 & DWR \\
\hline 1.2-Dibromoethane & $<0.5$ & $\mathrm{ug} / \mathrm{L}$ & 0.5 & SW8021 & $\mathrm{MDH}$ & $465 \mathrm{~F}$ & 14 & Dec 06 & DWR \\
\hline MethyI EthyI Ketone & $<5$ & $\mathrm{ug} / \mathrm{L}$ & 5 & SW8 021 & MDH & $465 \mathrm{~F}$ & 14 & Dec 06 & DWR \\
\hline Methyl Isobutyl Ketone & $<1.4$ & $\mathrm{ug} / \mathrm{L}$ & 1.4 & SWB 021 & $\mathrm{MDH}$ & $465 \mathrm{~F}$ & 14 & Dec 06 & DWR \\
\hline Tetrahydrofuran & $<5$ & ug/L & 5 & SWB 021 & MDH & $465 \mathrm{~F}$ & 14 & Dec 06 & DWR \\
\hline $\mathrm{m}$-xylene and $\mathrm{p}$-xylene & $<0.7$ & $\mathrm{ug} / \mathrm{L}$ & 0.7 & SW8 021 & $\mathrm{MDH}$ & $465 \mathrm{~F}$ & 14 & Dec 06 & DWR \\
\hline o-xylene & $<0.3$ & $\mathrm{ug} / \mathrm{L}$ & 0.3 & SWBO 21 & $\mathrm{MDH}$ & $465 \mathrm{~F}$ & 14 & Dec 06 & DWR \\
\hline
\end{tabular}

RL = Reporting Linit 
JARDA SOLC

ENERGY \& ENVIRONMENTAL RESEARCH CTR

UNIVERSITY OF NORTH DAKOTA

GRAND FORKS ND 58203

\section{Sample Description: FIELD BLANK} W5641
Page: 2 of 2

Report Date: 15 Dec 06

Lab Number: 06-A54141

work Order \#:82-2656

Account \#: 007033

Sample Matrix: GROUNDWATER

Date Sampled: 6 Dec 06

Date Received: 8 Dec 06

PO \#: CSI WATFORD

Temp at Receipt: $6.0 \mathrm{C}$

As Received Result

Method Method Date

RL Reference Analyzed

Analyst

\begin{tabular}{|c|c|c|c|c|c|c|c|c|c|c|}
\hline Cumene & $<0.7$ & $\mathrm{ug} / \mathrm{L}$ & 0.7 & SW8021 & $\mathrm{MDH}$ & $465 \mathrm{~F}$ & 24 & Dec & 06 & DWR \\
\hline $1,1,1,2$-Tetrachloroethane & $<0.5$ & $\mathrm{ug} / \mathrm{L}$ & 0.5 & SW8021 & $\mathrm{MDH}$ & $465 \mathrm{~F}$ & 14 & Dec & 06 & DWR \\
\hline 1.1-Dichloropropene & $<0.5$ & $\mathrm{ug} / \mathrm{L}$ & 0.5 & SW8021 & $\mathrm{MDH}$ & $465 \mathrm{~F}$ & 14 & Dec & 06 & DWR \\
\hline Dichlorofluoromethane & $<0.5$ & $\mathrm{ug} / \mathrm{L}$ & 0.5 & SW8021 & MDH & $465 \mathrm{~F}$ & 14 & Dec & 06 & DWR \\
\hline Trichlorotrifluoroethane & $<0.9$ & $\mathrm{ug} / \mathrm{L}$ & 0.9 & SW8021 & $\mathrm{MDH}$ & $465 \mathrm{~F}$ & 14 & Dec & 06 & DWR \\
\hline Ethyl Ether & $<0.8$ & $\mathrm{ug} / \mathrm{L}$ & 0.8 & SWB 021 & $\mathrm{MDH}$ & $465 \mathrm{~F}$ & 14 & Dec & 06 & DWR \\
\hline Acetone & $<10$ & $\mathrm{ug} / \mathrm{L}$ & 10 & SW8021 & $\mathrm{MDH}$ & $465 \mathrm{~F}$ & 14 & Dec & 06 & DWR \\
\hline Dibromomethane & $<0.7$ & $\operatorname{ug} / \mathrm{L}$ & 0.7 & SW8021 & $\mathrm{MDH}$ & $465 \mathrm{~F}$ & 14 & Dec & 06 & DWR \\
\hline 2,2-Dichloropropane & $<1$ & $\mathrm{ug} / \mathrm{L}$ & 1 & SW8021 & MDH & $465 \mathrm{~F}$ & 14 & Dec & 06 & DWR \\
\hline Bromochloromethane & $<0.6$ & $\mathrm{ug} / \mathrm{L}$ & 0.6 & SW8021 & $\mathrm{MDH}$ & $465 \mathrm{~F}$ & 14 & Dec & 06 & DWR \\
\hline Methyl tert-butyl Ether & $<0.6$ & $\mathrm{ug} / \mathrm{L}$ & 0.6 & SW8021 & $\mathrm{MDH}$ & $465 \mathrm{~F}$ & 14 & Dec & 06 & DWR \\
\hline Styrene & $<0.5$ & $\mathrm{ug} / \mathrm{L}$ & 0.5 & SW8021 & $\mathrm{MDH}$ & $465 \mathrm{~F}$ & 14 & Dec & 06 & DWR \\
\hline n-Propylbenzene & $<0.8$ & $\mathrm{ug} / \mathrm{L}$ & 0.8 & SW8021 & MDH & $465 \mathrm{~F}$ & 14 & Dec & 06 & DWR \\
\hline Bromobenzene & $<0.5$ & $\mathrm{ug} / \mathrm{L}$ & 0.5 & SW8021 & $\mathrm{MDH}$ & $465 \mathrm{~F}$ & 14 & Dec & 06 & DWR \\
\hline 2-Chlorotoluene & $<0.8$ & $\mathrm{ug} / \mathrm{L}$ & 0.8 & SW8021 & $\mathrm{MDH}$ & $465 \mathrm{~F}$ & 14 & Dec & 06 & DWR \\
\hline 1,3,5-Trimethylbenzene & $<0.9$ & $\mathrm{ug} / \mathrm{L}$ & 0.9 & SW8021 & MDH & $465 \mathrm{~F}$ & 14 & Dec & 06 & DWR \\
\hline 4-Chlorotoluene & $<0.6$ & $\mathrm{ug} / \mathrm{L}$ & 0.6 & SW8021 & MDH & $465 \mathrm{~F}$ & 14 & Dec & 06 & DWR \\
\hline t-Butylbenzene & $<0.6$ & $\mathrm{ug} / \mathrm{L}$ & 0.6 & SW8021 & MDH & $465 \mathrm{~F}$ & 14 & Dec & 06 & DWR \\
\hline 1,2,4-Trimethylbenzene & $<0.7$ & $\mathrm{ug} / \mathrm{L}$ & 0.7 & SW8021 & MDH & $465 \mathrm{~F}$ & 14 & Dec & 06 & DWR \\
\hline sec-Butylbenzene & $<0.7$ & $\mathrm{ug} / \mathrm{L}$ & 0.7 & SW8021 & $\mathrm{MDH}$ & $465 \mathrm{~F}$ & 14 & Dec & 06 & DWR \\
\hline p-Isopropyltoluene & $<0.7$ & $\mathrm{ug} / \mathrm{L}$ & 0.7 & SW8021 & MDH & $465 \mathrm{~F}$ & 14 & Dec & 06 & DWR \\
\hline n-Butylbenzene & $<0.7$ & $\mathrm{ug} / \mathrm{L}$ & 0.7 & SW8021 & $\mathrm{MDH}$ & $465 \mathrm{~F}$ & 14 & Dec & 06 & DWR \\
\hline 1,2-Dibromo-3-chloropropane & $<1$ & $\mathrm{ug} / \mathrm{L}$ & 1 & SW8021 & MDH & $465 \mathrm{~F}$ & 14 & Dec & 06 & DWR \\
\hline 1,2,4-Trichlorobenzene & $<0.5$ & $\mathrm{ug} / \mathrm{L}$ & 0.5 & SW8021 & $\mathrm{MDH}$ & $465 \mathrm{~F}$ & 14 & Dec & 06 & DWR \\
\hline Hexachlorobutadiene & $<0.7$ & $\mathrm{ug} / \mathrm{L}$ & 0.7 & SW8021. & MDH & $465 \mathrm{~F}$ & 14 & Dec & 06 & DWR \\
\hline Naphthalene & $<1$ & $\mathrm{ug} / \mathrm{L}$ & 1 & SW8021 & $\mathrm{MDH}$ & $465 \mathrm{~F}$ & 14 & Dec & 06 & DWR \\
\hline 1,2,3-Trichlorobenzene & $<0.6$ & $\mathrm{ug} / \mathrm{L}$ & 0.6 & SW8021 & $\mathrm{MDH}$ & $465 \mathrm{~F}$ & 14 & Dec & 06 & DWR \\
\hline GRO (TPH) & $<0.2$ & $\mathrm{mg} / \mathrm{L}$ & 0.2 & $8015 B / C$ & $\mathrm{DAI}$ & & 8 & Dec & 06 & RDC \\
\hline
\end{tabular}

** No collection time supplied by the client.

GC VOC sample $\mathrm{pH}<2$

1-CHLORO-4-FLUOROBENZENE (SURROGATE) RECOVERY: $99 \%$

BTEX/GRO Sample $\mathrm{pH}<2$

BTEX SURROGATE RECOVERY: $105 \%$

GRO SURROGATE RECOVERY: $94 \div$
RL = Reporting Limit

Elevated "Less Than Result" (<): \# = Due to sample matrix : Due to sample quantity
$\#=$ Due to sample concentration

- Due to extract volume 
JARDA SOLC

ENERGY \& ENVIRONMENTAL RESEARCH CTR

UNIVERSITY OF NORTH DAKOTA

GRAND FORKS ND 58203

\section{Sample Description: EQUIPMENT BLANK W5642

\begin{tabular}{|c|c|c|c|c|c|c|c|c|c|}
\hline \multirow[b]{2}{*}{ Chloroethane } & \multicolumn{2}{|c|}{$\begin{array}{l}\text { As Received } \\
\text { Result }\end{array}$} & \multirow{2}{*}{$\begin{array}{l}\text { Method } \\
\text { RL } \\
0.7\end{array}$} & \multicolumn{3}{|c|}{$\begin{array}{l}\text { Method } \\
\text { Reference }\end{array}$} & \multicolumn{2}{|c|}{$\begin{array}{l}\text { Date } \\
\text { Analyzed }\end{array}$} & \multirow{2}{*}{$\frac{\text { Analyst }}{\text { DWR }}$} \\
\hline & $<0.7$ & $\mathrm{ug} / \mathrm{L}$ & & SWB 021 & $\mathrm{MDH}$ & $465 \mathrm{~F}$ & 14 & Dec 06 & \\
\hline Chloromethane & $<1$ & $\mathrm{ug} / \mathrm{L}$ & 1 & SWB 021 & MDH & $465 \mathrm{~F}$ & 14 & Dec 06 & DWR \\
\hline Bromomethane & $<0.7$ & $\mathrm{ug} / \mathrm{L}$ & 0.7 & SW8021 & $\mathrm{MDH}$ & $465 \mathrm{~F}$ & 14 & Dec 06 & DWR \\
\hline Dichlorodifluoromethane & $<0.9$ & $u g / L$ & 0.9 & SW8021 & MDH & $465 \mathrm{~F}$ & 14 & Dec 06 & DWR \\
\hline Vinyl chloride & $<0.5$ & $\mathrm{ug} / \mathrm{L}$ & 0.5 & SW8 021 & $\mathrm{MDH}$ & $465 \mathrm{~F}$ & 14 & Dec 06 & DWR \\
\hline Methylene Chloride & $<0.7$ & $\mathrm{ug} / \mathrm{L}$ & 0.7 & SW8021 & MDH & $465 \mathrm{~F}$ & 14 & Dec 06 & DWR \\
\hline Trichlorof luoromethane & $<0.9$ & $\mathrm{ug} / \mathrm{L}$ & 0.9 & SW8021 & MDH & $465 \mathrm{~F}$ & 14 & Dec 06 & DWR \\
\hline 1.I-Dichloroethene & $<0.5$ & $\mathrm{ug} / \mathrm{L}$ & 0.5 & SW8021 & MDH & $465 \mathrm{~F}$ & 14 & Dec 06 & DWR \\
\hline 1,1-Dichloroethane & $<0.8$ & $\mathrm{ug} / \mathrm{L}$ & 0.8 & SW8021. & MDH & $465 \mathrm{~F}$ & 1.4 & Dec 06 & DWR \\
\hline trans-1,2-Dichloroethene & $<0.5$ & $\mathrm{ug} / \mathrm{I}$ & 0.5 & SW8021 & MDH & $465 \mathrm{~F}$ & 14 & Dec 06 & DWR \\
\hline Chloro form & $<0.8$ & $\mathrm{ug} / \mathrm{L}$ & 0.8 & SW8021 & MDH & $465 \mathrm{~F}$ & 14 & Dec 06 & DWR \\
\hline 1,2-Dichloroethane & $<0.5$ & $\mathrm{ug} / \mathrm{L}$ & 0.5 & SW8 021 & $\mathrm{MDH}$ & $465 \mathrm{~F}$ & 14 & Dec 06 & DWR \\
\hline 1,1.1-Trichloroethane & $<0.9$ & $\mathrm{ug} / \mathrm{L}$ & 0.9 & Sw802I & $\mathrm{MDH}$ & $465 \mathrm{~F}$ & 14 & Dec 06 & DWR \\
\hline Carbon Tetrachloride & $<0.8$ & $\mathrm{ug} / \mathrm{L}$ & 0.8 & SW8021 & MDH & $465 \mathrm{~F}$ & 1.4 & Dec 06 & DWR \\
\hline Bromodichloromethane & $<0.5$ & $\mathrm{ug} / \mathrm{L}$ & 0.5 & SW8021 & MDH & $465 \mathrm{~F}$ & 14 & Dec 06 & DWR \\
\hline 1,2-Dichloropropane & $<0.7$ & $\mathrm{ug} / \mathrm{L}$ & 0.7 & SW8021 & $\mathrm{MDH}$ & $465 \mathrm{~F}$ & 14 & Dec 06 & DWR \\
\hline trans-1,3-Dichloropropene & $<0.5$ & $\mathrm{ug} / \mathrm{L}$ & 0.5 & Sw8021 & MDH & $465 \mathrm{~F}$ & 14 & Dec 06 & DWR \\
\hline Trichloroethene & $<0.5$ & $\mathrm{ug} / \mathrm{L}$ & 0.5 & SW8021 & MDH & $465 \mathrm{~F}$ & 14 & Dec 06 & DWR \\
\hline Chlorodibromomethane & $<0.5$ & $\mathrm{ug} / \mathrm{L}$ & 0.5 & SW8021 & MDH & $465 \mathrm{~F}$ & 14 & Dec 06 & DWR \\
\hline 1.1.2-Trichloroethane & $<0.6$ & $\mathrm{ug} / \mathrm{L}$ & 0.6 & SWB021 & $\mathrm{MDH}$ & $465 \mathrm{~F}$ & 14 & Dec 06 & DWR \\
\hline cis-1,3-Dichloropropene & $<0.5$ & $\mathrm{ug} / \mathrm{L}$ & 0.5 & SWBO2I & MDH & $465 \mathrm{~F}$ & 14 & Dec 06 & DWR \\
\hline Bromoform & $<1$ & $\mathrm{ug} / \mathrm{L}$ & 1 & Sw8021 & MDH & $465 \mathrm{~F}$ & 14 & Dec 06 & DWR \\
\hline $1,1,2,2$-Tetrachloroethane & $<0.5$ & $\mathrm{ug} / \mathrm{L}$ & 0.5 & SW8021 & $\mathrm{MDH}$ & $465 \mathrm{~F}$ & 14 & Dec 06 & DWR \\
\hline Tetrachloroethene & $<0.7$ & $\mathrm{ug} / \mathrm{L}$ & 0.7 & SWB 021 & $\mathrm{MDH}$ & $465 \mathrm{~F}$ & 14 & Dec 06 & DWR \\
\hline Chlorobenzene & $<0.6$ & $\mathrm{ug} / \mathrm{L}$ & 0.6 & SW802I & $\mathrm{MDH}$ & $465 \mathrm{~F}$ & 14 & Dec of & DWR \\
\hline Benzene & $<0.5$ & $\mathrm{ug} / \mathrm{L}$ & 0.5 & SW8021 & $\mathrm{MDH}$ & $465 \mathrm{~F}$ & 14 & Dec 06 & DWR \\
\hline Toluene & $<0.6$ & $\mathrm{ug} / \mathrm{L}$ & 0.6 & SW802I & $\mathrm{MDH}$ & $465 \mathrm{~F}$ & 14 & Dec 06 & DWR \\
\hline Ethyl Benzene & $<0.6$ & $\mathrm{ug} / \mathrm{L}$ & 0.6 & SW8021 & $\mathrm{MDH}$ & $465 \mathrm{~F}$ & 14 & Dec 06 & DWR \\
\hline 1.2-Dichlorobenzene & $<0.5$ & $\mathrm{ug} / \mathrm{L}$ & 0.5 & SW8021 & $\mathrm{MDH}$ & $465 \mathrm{~F}$ & 14 & Dec 06 & DWR \\
\hline 1,3-Dichlorobenzene & $<0.5$ & $\mathrm{ug} / \mathrm{L}$ & 0.5 & SW8021 & $\mathrm{MDH}$ & $465 \mathrm{~F}$ & 14 & Dec 06 & DWR \\
\hline 1,4-Dichlorobenzene & $<0.5$ & $\mathrm{ug} / \mathrm{L}$ & 0.5 & SW8021 & $\mathrm{MDH}$ & $465 \mathrm{~F}$ & 14 & Dec 06 & DWR \\
\hline cis-1,2-Dichloroethene & $<0.5$ & $\mathrm{ug} / \mathrm{L}$ & 0.5 & SW8 021 & $\mathrm{MDH}$ & $465 \mathrm{~F}$ & 14 & Dec 06 & DWR. \\
\hline 1,3-Dichloropropane & $<0.6$ & $\mathrm{ug} / \mathrm{L}$ & 0.6 & SW8021 & $\mathrm{MDH}$ & $465 \mathrm{~F}$ & 14 & DeC 06 & DWR \\
\hline 1,2,3-Trichloropropane & $<0.5$ & $\mathrm{ug} / \mathrm{L}$ & 0.5 & SW8 021 & $\mathrm{MDH}$ & $465 \mathrm{~F}$ & 14 & Dec 06 & DWR \\
\hline Allyl Chloride & $<0.7$ & $\mathrm{ug} / \mathrm{L}$ & 0.7 & SW8 021 & $\mathrm{MDH}$ & $465 \mathrm{~F}$ & 14 & Dec 06 & DWR \\
\hline 1,2 -Dibromoethane & $<0.5$ & $\mathrm{ug} / \mathrm{L}$ & 0.5 & SW8 021 & MDH & $465 \mathrm{~F}$ & 14 & Dec 06 & DWR \\
\hline Methyl Ethyl Ketone & $<5$ & $\mathrm{ug} / \mathrm{L}$ & 5 & SW8 021 & MDH & $465 \mathrm{~F}$ & 14 & Dec 06 & DWR \\
\hline Methyl Isobutyl Ketone & $<1.4$ & $\mathrm{ug} / \mathrm{L}$ & 1.4 & SW8021 & $\mathrm{MDH}$ & $465 \mathrm{~F}$ & 14 & Dec 06 & DWR \\
\hline Tetrahydrofuran & $<5$ & $\mathrm{ug} / \mathrm{L}$ & 5 & SW8021 & $\mathrm{MDH}$ & $465 \mathrm{~F}$ & 14 & Dec 06 & DWR \\
\hline$m$-xylene and $p$-xylene & $<0.7$ & $\mathrm{ug} / \mathrm{L}$ & 0.7 & SW8021 & $\mathrm{MDH}$ & $465 \mathrm{~F}$ & 14 & Dec 06 & DWR \\
\hline & $<0.3$ & $\mathrm{ug} / \mathrm{L}$ & 0.3 & SW8021 & $\mathrm{MDH}$ & $465 \mathrm{~F}$ & 14 & Dec 06 & DWR \\
\hline
\end{tabular}

Page: 1 of 2

Report Date: 15 Dec 06

Lab Number: 06-A54142

Work Order \#:82-2656

Account \#: 007033

Sample Matrix: GROUNDWATER

Date Sampled: 6 Dec 06

Date Received: 8 Dec 06

PO \# : CSI WATFORD

Temp at Receipt: $6.0 \mathrm{C}$ 
JARDA SOLC

ENERGY \& ENVIRONMENTAL RESEARCH CTR

UNIVERSITY OF NORTH DAKOTA

GRAND FORKS ND 58203

\section{Sample Description: EQUIPMENT BLANK W5642}

As Received

Result

Cumene

$1,1,1,2$-Tetrachloroethane

$<0.7$

$<0.5$

$<0.5$

$<0.5$

Dichlorofluoromethane

Trichlorotrifluoroethane

Ethyl Ether

Acetone

Dibromomethane

2,2-Dichloropropane

Bromochloromethane

Methyl tert-butyl Ether

styrene

n-Propylbenzene

Bromobenzene

2-Chlorotoluene

1, 3, 5-Trimethylbenzene

4-Chlorotoluene

$\mathrm{t}$-Butylbenzene

1,2,4-Trimethylbenzene

sec-Butylbenzene

p-I sopropyltoluene

n-Butylbenzene

1,2-Dibromo-3-chloropropane

1,2,4-Trichlorobenzene

Hexachlorobutadiene

Naphthalene

1,2,3-Trichlorobenzene

GRO (TPH)

$<0.8$

$<10$

$<0.7$

$<1$

$<0.6$
$<0.6$

$<0.5$

$<0.8$

$<0.5$

$<0.8$

$<0.9$

$<0.6$

$<0.6$

$<0.7$

$<0.7$

$<0.7$

4.7

$<1$

$<0.5$

0.7

$<1$

$<0.6$

$<0.2$

$\mathrm{ug} / \mathrm{L}$
$\mathrm{ug} / \mathrm{L}$
$\mathrm{ug} / \mathrm{L}$
$\mathrm{ug} / \mathrm{L}$
$\mathrm{ug} / \mathrm{L}$
$\mathrm{ug} / \mathrm{L}$
$\mathrm{ug} / \mathrm{L}$
$\mathrm{ug} / \mathrm{L}$
$\mathrm{ug} / \mathrm{L}$
$\mathrm{ug} / \mathrm{L}$
$\mathrm{ug} / \mathrm{L}$
$\mathrm{ug} / \mathrm{L}$
$\mathrm{ug} / \mathrm{L}$
$\mathrm{ug} / \mathrm{L}$
$\mathrm{ug} / \mathrm{L}$
$\mathrm{ug} / \mathrm{L}$
$\mathrm{ug} / \mathrm{L}$
$\mathrm{ug} / \mathrm{L}$
$\mathrm{ug} / \mathrm{L}$
$\mathrm{ug} / \mathrm{L}$
$\mathrm{ug} / \mathrm{L}$
$\mathrm{ug} / \mathrm{L}$
$\mathrm{ug} / \mathrm{L}$
$\mathrm{ug} / \mathrm{L}$
$\mathrm{ug} / \mathrm{L}$
$\mathrm{ug} / \mathrm{L}$
$\mathrm{ug} / \mathrm{L}$
$\mathrm{mg} / \mathrm{L}$

* No collection time supplied by the client.

GC VoC Sample $\mathrm{pH}<2$

1-CHLORO-4-FLUOROBENZENE (SURROGATE) RECOVERY: $100 \%$

BTEX/GRO Sample pH $<2$

BTEX SURROGATE RECOVERY: $104 \stackrel{\circ}{\mathrm{n}}$

GRO SURROGATE RECOVERY: $98 \%$
Page: 2 of 2

Report Date: 15 Dec 06

Lab Number: 06-A54142

Work Order \#:82-2656

Account \#: 007033

Sample Matrix: GROUNDWATER

Date Sampled: 6 Dec 06

Date Received: 8 Dec 06

PO \#: CSI WATFORD

Temp at Receipt: $6.0 \mathrm{C}$ 
JARDA SOLC

ENERGY \& ENVIRONMENTAL RESEARCH CTR

UNIVERSITY OF NORTH DAKOTA

GRAND FORKS ND 58203

Sample Description: TRIP BLANK

As Received

Result

$<0.7$

$<1$

$<0.7$

$<0.9$

$<0.5$

$<0.7$

$<0.9$

$<0.5$

$<0.8$

$<0.5$

$<0.8$

$<0.5$

$<0.9$

$<0.8$

$<0.5$

$<0.7$

$<0.5$

$<0.5$

$<0.5$

$<0.6$

$<0.5$

$<1$

$<0.5$

$<0.7$

$<0.6$

$<0.5$

$<0.6$

$<0.6$

$<0.5$

$<0.5$

$<0.5$

$<0.5$

$<0.6$

$<0.5$

$<0.7$

$<0.5$

$<5$

$<1.4$

$<5$

$<0.7$

$<0.3$ $\mathrm{ug} / \mathrm{L}$

ug/L

$\mathrm{ug} / \mathrm{L}$

$\mathrm{ug} / \mathrm{L}$

$\mathrm{ug} / \mathrm{L}$

ug/L

ug/L

ug/L

ug/L

$\mathrm{ug} / \mathrm{L}$

$\mathrm{ug} / \mathrm{L}$

$$
\text { ug/L }
$$

ug/L

$u g / L$

$$
\text { ug/L }
$$

ug/L,

ug/L

$$
\mathrm{ug} / \mathrm{L}
$$

$\mathrm{ug} / \mathrm{L}$

ug/L

$$
\text { ug } / \mathrm{L}
$$

ug/L

ug/L

$\mathrm{ug} / \mathrm{L}$

$\mathrm{ug} / \mathrm{L}$

\section{ug $/ \mathrm{L}$}

$\mathrm{ug} / \mathrm{L}$$$
\text { ug/L }
$$

$\mathrm{ug} / \mathrm{L}$

$\mathrm{ug} / \mathrm{L}$

ug/L.

$\mathrm{ug} / \mathrm{L}$

ug/L

$\mathrm{ug} / \mathrm{L}$

$\mathrm{ug} / \mathrm{L}$

$\mathrm{ug} / \mathrm{L}$

$\mathrm{ug} / \mathrm{L}$

$\mathrm{ug} / \mathrm{L}$

$\mathrm{ug} / \mathrm{L}$ $\mathrm{m}-\mathrm{xy}$ lene and $\mathrm{p}$-xylene o-Xylene
Page: 1 of 2

Report Date: 15 Dec 06

Lab Number: 06-A54143

Work Order \#:82-2656

Account \#: 007033

Sample Matrix: GROUNDWATER

Date Sampled: 6 Dec 06

\begin{tabular}{|c|c|c|c|}
\hline $\begin{array}{l}\text { Method } \\
\text { RL }\end{array}$ & $\begin{array}{l}\text { Method } \\
\text { Referer }\end{array}$ & & \\
\hline 0.7 & SWBO21 & $\mathrm{MDH}$ & $465 \mathrm{~F}$ \\
\hline 1 & SW802I & $\mathrm{MDH}$ & $465 \mathrm{~F}$ \\
\hline 0.7 & SW8021 & MDH & $465 \mathrm{~F}$ \\
\hline 0.9 & SW8021 & $\mathrm{MDH}$ & $465 \mathrm{~F}$ \\
\hline 0.5 & SW8021 & $\mathrm{MDH}$ & $465 \mathrm{~F}$ \\
\hline 0.7 & SW8021 & MDH & $465 \mathrm{~F}$ \\
\hline 0.9 & SW8021 & $\mathrm{MDH}$ & $465 \mathrm{~F}$ \\
\hline 0.5 & Sw8021 & $\mathrm{MDH}$ & $465 F$ \\
\hline 0.8 & SW8021 & $\mathrm{MDH}$ & $465 F$ \\
\hline 0.5 & SWB02I & $\mathrm{MDH}$ & $465 \mathrm{~F}$ \\
\hline 0.8 & SW8021 & $\mathrm{MDH}$ & $465 \mathrm{~F}$ \\
\hline 0.5 & SW8021 & $\mathrm{MDH}$ & $465 \mathrm{~F}$ \\
\hline 0.9 & SW8021 & $\mathrm{MDH}$ & $465 F$ \\
\hline 0.8 & SW8021 & $\mathrm{MDH}$ & $465 \mathrm{~F}$ \\
\hline 0.5 & SW8021 & MDH & $465 \mathrm{~F}$ \\
\hline 0.7 & SW8021 & $\mathrm{MDH}$ & $465 \mathrm{~F}$ \\
\hline 0.5 & SW8021 & $\mathrm{MDH}$ & $465 \mathrm{~F}$ \\
\hline 0.5 & SW8021 & $\mathrm{MDH}$ & $465 F$ \\
\hline 0.5 & SW8021 & $\mathrm{MDH}$ & $465 F$ \\
\hline 0.6 & SW8021 & MDH & $465 F$ \\
\hline 0.5 & SW8021 & $\mathrm{MDH}$ & $465 \mathrm{~F}$ \\
\hline 1 & SW8021 & $\mathrm{MDH}$ & $465 \mathrm{~F}$ \\
\hline 0.5 & SW8021 & $\mathrm{MDH}$ & $465 \mathrm{~F}$ \\
\hline 0.7 & SW8021 & MDH & $465 \mathrm{~F}$ \\
\hline 0.6 & SW8021 & MDH & $465 \mathrm{~F}$ \\
\hline 0.5 & SW8021 & $\mathrm{MDH}$ & $465 \mathrm{~F}$ \\
\hline 0.6 & SW8021 & $\mathrm{MDH}$ & $465 \mathrm{~F}$ \\
\hline 0.6 & SWBO21 & $\mathrm{MDH}$ & $465 \mathrm{~F}$ \\
\hline 0.5 & SW8021 & $\mathrm{MDH}$ & $465 \mathrm{~F}$ \\
\hline 0.5 & SWBO2I & MDH & $465 \mathrm{~F}$ \\
\hline 0.5 & SW802I & $\mathrm{MDH}$ & $465 \mathrm{~F}$ \\
\hline 0.5 & SWB021 & $\mathrm{MDH}$ & $465 \mathrm{~F}$ \\
\hline 0.6 & SW8021 & MDH & $465 \mathrm{~F}$ \\
\hline 0.5 & Sw8021 & $\mathrm{MDH}$ & $465 \mathrm{~F}$ \\
\hline 0.7 & SW8021 & $\mathrm{MDH}$ & $465 \mathrm{~F}$ \\
\hline 0.5 & SW8021 & $\mathrm{MDH}$ & $465 \mathrm{~F}$ \\
\hline 5 & SW8021 & $\mathrm{MDH}$ & $465 \mathrm{~F}$ \\
\hline 1.4 & SW8021 & $\mathrm{MDH}$ & $465 \mathrm{~F}$ \\
\hline 5 & SW8021 & $\mathrm{MDH}$ & $465 \mathrm{~F}$ \\
\hline 0.7 & SW8021 & $\mathrm{MDH}$ & $465 \mathrm{~F}^{2}$ \\
\hline 0.3 & SW8021 & $\mathrm{MDH}$ & $465 \mathrm{~F}$ \\
\hline
\end{tabular}

Date Received: 8 Dec 06

PO \#: CSI WATFORD

Temp at Receipt: $6.0 \mathrm{C}$
Date

Analyzed

Analyst

14 Dec 06

14 DeC 06

14 DeC 06

14 Dec 06

14 Dec 06

14 Dec 06

14 Dec 06

14 DeC 06

14 Dec 06

14 DeC 06

14 DeC 06

14 Dec 06

14 Dec 06

14 DeC 06

14 DeC 06

14 Dec 06

14 DeC 06

14 DeC 06

14 Dec 06

14 DeC 06

14 Dec 06

14 Dec 06

14 Dec 06

14 Dec 06

14 Dec 06

14 DeC 06

14 DeC 06

14 Dec 06

14 DeC 06

14 Dec 06

14 DeC 06

14 DeC 06

14 DeC 06

14 Dec 06

14 Dec 06

14 DeC 06

14 DeC 06

14 Dec 06

14 Dec 06

14 Dec 06

14 Dec 06

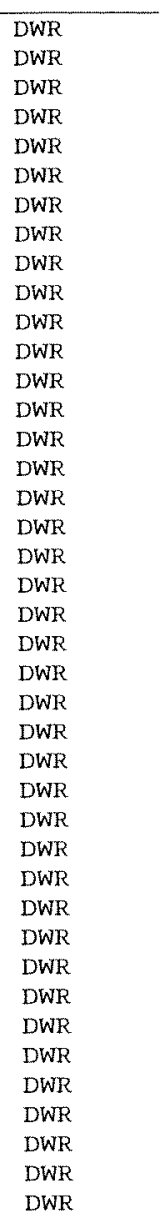

RL = Reporting Limit 
JARDA SOLC

ENERGY \& ENVIRONMENTAL RESEARCH CTR

UNIVERSITY OF NORTH DAKOTA

GRAND FORKS ND 58203

Sample Description: TRIP BLANK

\begin{tabular}{|c|c|c|c|c|c|c|c|c|c|}
\hline \multirow[b]{2}{*}{ Cumene } & \multicolumn{2}{|c|}{$\begin{array}{l}\text { As Received } \\
\text { Result }\end{array}$} & \multirow{2}{*}{$\begin{array}{l}\text { Method } \\
\text { RL } \\
0.7\end{array}$} & \multicolumn{3}{|c|}{$\begin{array}{l}\text { Method } \\
\text { Reference }\end{array}$} & \multicolumn{2}{|c|}{ Date } & \multirow{2}{*}{$\begin{array}{l}\text { Analyst } \\
\text { DWR }\end{array}$} \\
\hline & $<0.7$ & $\mathrm{ug} / \mathrm{L}$ & & SW8021 & MDH & $465 F$ & 14 & Dec 06 & \\
\hline 1,1,1,2-Tetrachloroethane & $<0.5$ & $\mathrm{ug} / \mathrm{L}$ & 0.5 & SW8021 & MDH & $465 \mathrm{~F}$ & 14 & Dec 06 & DWR \\
\hline 1.1-Dichloropropene & $<0.5$ & $\mathrm{ug} / \mathrm{L}$ & 0.5 & SW8021 & $\mathrm{MDH}$ & $465 \mathrm{~F}$ & 14 & Dec 06 & DWR \\
\hline Dichlorof luoromethane & $<0.5$ & $\mathrm{ug} / \mathrm{L}$ & 0.5 & SW8021 & $\mathrm{MDH}$ & $465 \mathrm{~F}$ & 14 & Dec 06 & DWR \\
\hline Trichlorotrifluoroethane & $<0.9$ & $\mathrm{ug} / \mathrm{L}$ & 0.9 & SW8021 & $\mathrm{MDH}$ & $465 \mathrm{~F}$ & 14 & Dec 06 & DWR \\
\hline Ethyl Ether & $<0.8$ & $\mathrm{ug} / \mathrm{L}$ & 0.8 & SW8021 & $\mathrm{MDH}$ & $465 \mathrm{~F}$ & 14 & Dec 06 & DWR \\
\hline Acetone & $<10$ & $\mathrm{ug} / \mathrm{L}$ & 10 & Sw8 021 & $\mathrm{MDH}$ & $465 \mathrm{~F}$ & 14 & Dec 06 & DWR \\
\hline Dibromomethane & $<0.7$ & $\mathrm{ug} / \mathrm{L}$ & 0.7 & Sw8 021 & $\mathrm{MDH}$ & $465 \mathrm{~F}$ & 14 & Dec 06 & DWR \\
\hline 2,2-Dichloropropane & $<1$ & $\mathrm{ug} / \mathrm{L}$ & 1 & SW8021 & MDH & $465 \mathrm{~F}$ & 14 & Dec 06 & DWR \\
\hline Bromochloromethane & $<0.6$ & $\mathrm{ug} / \mathrm{L}$ & 0.6 & SW802I & MDH & $465 \mathrm{~F}$ & 14 & Dec 06 & DWR \\
\hline Methyl tert-butyl Ether & $<0.6$ & $\mathrm{ug} / \mathrm{L}$ & 0.6 & SW8021 & $\mathrm{MDH}$ & $465 \mathrm{~F}$ & 14 & Dec 06 & DWR \\
\hline Styrene & $<0.5$ & $\mathrm{ug} / \mathrm{L}$ & 0.5 & SW8021 & $\mathrm{MDH}$ & $465 \mathrm{~F}$ & 14 & Dec 06 & DWR \\
\hline n-Propylbenzene & $<0.8$ & $\mathrm{ug} / \mathrm{L}$ & 0.8 & Sw8 021 & $\mathrm{MDH}$ & $465 \mathrm{~F}$ & 14 & Dec 06 & DWR \\
\hline Bromobenzene & $<0.5$ & $\mathrm{ug} / \mathrm{L}$ & 0.5 & SW8021 & MDH & $465 \mathrm{~F}$ & 14 & Dec 06 & DWR \\
\hline 2-chlorotoluene & $<0.8$ & $\mathrm{ug} / \mathrm{L}$ & 0.8 & SW8021 & MDH & $465 \mathrm{~F}$ & 14 & Dec 06 & DWR \\
\hline 1,3,5-Trimethylbenzene & $<0.9$ & $\mathrm{ug} / \mathrm{L}$ & 0.9 & SWB021 & MDH & $465 \mathrm{~F}$ & 14 & Dec 06 & DWR \\
\hline 4-Chlorotoluene & $<0.6$ & $\mathrm{ug} / \mathrm{L}$ & 0.6 & Sw8 021 & $\mathrm{MDH}$ & $465 \mathrm{~F}$ & 14 & Dec 06 & DWR \\
\hline t-Butylbenzene & $<0.6$ & $\mathrm{ug} / \mathrm{L}$ & 0.6 & SW8021 & $\mathrm{MDH}$ & $465 \mathrm{~F}$ & 14 & Dec 06 & DWR \\
\hline 1,2,4-Trimethylbenzene & $<0.7$ & $\mathrm{ug} / \mathrm{L}$ & 0.7 & SW8021 & $\mathrm{MDH}$ & $465 \mathrm{~F}$ & 14 & Dec 06 & DWR \\
\hline sec-Butylbenzene & $<0.7$ & $\mathrm{ug} / \mathrm{L}$ & 0.7 & SWB021 & $\mathrm{MDH}$ & $465 \mathrm{~F}$ & 14 & Dec 06 & DWR \\
\hline p-Isopropyltoluene & $<0.7$ & $\mathrm{ug} / \mathrm{L}$ & 0.7 & SWB021 & MDH & $465 \mathrm{~F}$ & 14 & Dec 06 & DWR \\
\hline n-Butylbenzene & $<0.7$ & $\mathrm{ug} / \mathrm{L}$ & 0.7 & SW8021 & $\mathrm{MDH}$ & $465 \mathrm{~F}$ & 14 & Dec 06 & DWR \\
\hline 1,2-Dibromo-3-chloropropane & $<1$ & $\mathrm{ug} / \mathrm{L}$ & 1 & SWB 021 & $\mathrm{MDH}$ & $465 \mathrm{~F}$ & 14 & Dec 06 & DWR \\
\hline 1,2,4-Trichlorobenzene & $<0.5$ & $\mathrm{ug} / \mathrm{L}$ & 0.5 & SW8021 & MDH & $465 \mathrm{~F}$ & 14 & Dec 06 & DWR \\
\hline Hexachlorobutadiene & $<0.7$ & $\mathrm{ug} / \mathrm{L}$ & 0.7 & SW8021 & MDH & $465 \mathrm{~F}$ & 14 & Dec 06 & DWR \\
\hline Naphthalene & $<1$ & $\mathrm{ug} / \mathrm{L}$ & 1 & SW8021 & $\mathrm{MDH}$ & $465 \mathrm{~F}$ & 14 & Dec 06 & DWR \\
\hline 1,2,3-Trichlorobenzene & $<0.6$ & $\mathrm{ug} / \mathrm{L}$ & 0.6 & SW8021 & $\mathrm{MDH}$ & $465 \mathrm{~F}$ & 14 & Dec 06 & DWR \\
\hline GRO (TPH) & $<0.2$ & $\mathrm{mg} / \mathrm{L}$ & 0.2 & $8015 B / C$ & OAI & & 8 & Dec 06 & RDC \\
\hline
\end{tabular}

** No collection time supplied by the client.

GC VOC Sample $\mathrm{pH}<2$

1-CHLORO-4-FLUOROBENZENE (SURROGATE) RECOVERY: $100 \div$

BTEX/GRO Sample $\mathrm{pH}<2$

BTEX SURROGATE RECOVERY: $104 \%$

GRO SURROGATE RECOVERY : $95 \%$
Page: 2 of 2

Report Date: 15 Dec 06

Lab Number: 06-A54143

Work Order \#:82-2656

Account \#: 007033

Sample Matrix: GROUNDWATER

Date Sampled: 6 Dec 06

Date Received: 8 Dec 06

PO \# : CSI WATFORD

Temp at Receipt: $6.0 \mathrm{C}$ 
APPENDIX G-2

\section{BIODEGRADATION INDICATORS}


Jarda Solc

UND-Energy \& Environmental

$15 \mathrm{~N} .23 \mathrm{rd}$ st.

Grand Forks ND 58201

Sample Description: MW-1

Sample site: Watford City
Page: 1 of 1

Report Date: 22 Dec 06

Lab Number: $06-$ W5630

work Order \#: 82-2656

Account \#: 007033

Date Sampled: 6 Dec 06 8:45

Date Received: 7 Dec 06 8:10

PO \#: CSI Watford

\begin{tabular}{|c|c|c|c|c|c|c|c|c|c|}
\hline \multirow{4}{*}{$\begin{array}{l}\text { Biochemical Oxygen Demand } \\
\text { Total organic Carbon } \\
\text { Sulfate }\end{array}$} & \multicolumn{2}{|c|}{$\begin{array}{l}\text { As Received } \\
\text { Result }\end{array}$} & \multirow{2}{*}{$\begin{array}{l}\text { Method } \\
\text { RL } \\
2\end{array}$} & \multirow{2}{*}{$\begin{array}{l}\begin{array}{l}\text { Method } \\
\text { Reference }\end{array} \\
\text { SM } 5210-B\end{array}$} & \multicolumn{4}{|c|}{$\begin{array}{l}\text { Date } \\
\text { Analyzed }\end{array}$} & \multirow{2}{*}{ Analyst } \\
\hline & $<2$ & $\mathrm{mg} / 1$ & & & 7 & Dec & 06 & $8: 45$ & \\
\hline & 27.0 & $\mathrm{mg} / \mathrm{l}$ & 0.5 & SM5310-C & 11 & Dec & 06 & $8: 30$ & Wayne \\
\hline & 8500 & $\mathrm{mg} / \mathrm{I}$ & 5.00 & EPA 375.4 & 12 & Dec & 06 & $15: 31$ & Morgan \\
\hline Nitrate-Nitrite as $N$ & 0.18 & $\mathrm{mg} / \mathrm{l}$ & 0.10 & 353.2 & 13 & Dec & 06 & $9: 00$ & Morgan \\
\hline Ammonia-Nitrogen as $N$ & $<0.1$ & $\mathrm{mg} / \mathrm{I}$ & 0.10 & EPA 350.1 & 15 & Dec & 06 & $10: 00$ & Morgan \\
\hline Phosphorus as $\mathrm{P}$ - Total & 0.16 & $\mathrm{mg} / \mathrm{I}$ & 0.10 & EPA 365.1 & 11. & Dec & 06 & $12: 00$ & Morgan \\
\hline Chemical Oxygen Demand & 63.6 & $\mathrm{mg} / 1$ & 1.0 & HACH 8000 & 8 & Dec & 06 & $8: 30$ & wayñe \\
\hline Iron - Total & 37.4 & $\mathrm{mg} / 1$ & 0.10 & 6010 & 21 & Dec & 06 & $9: 12$ & stacy \\
\hline Manganese - Total & 7.80 & $\mathrm{mg} / \mathrm{I}$ & 0.05 & 6010 & 21 & Dec & 06 & $9: 12$ & stacy \\
\hline Iron - Dissol.ved & $<0.1$ & $\mathrm{mg} / \mathrm{I}$ & 0.10 & 6010 & 14 & Dec & 06 & $9: 41$ & stacy \\
\hline Manganese - Dissolved & $<0.05$ & $\mathrm{mg} / 1$ & 0.05 & 6010 & 14 & Dec & 06 & $9: 41$ & Stacy \\
\hline
\end{tabular}

Approved by: 
Jarda Solc

UND-Energy \& Environmental

15 N. 23 rd St.

Grand Forks ND 58201

Sample Description: MW- 5

Sample site: Watford City
Page: 1 of 1

Report Date: 22 Dec 06

Lab Number: 06-W5631

Work Ordex \#: $82-2656$

Account \#: 007033

Date Sampled: 6 Dec 06 15:15

Date Received: 7 Dec $06 \quad 8: 10$

PO \#: CSI Watford

\begin{tabular}{|c|c|c|c|c|c|c|c|c|c|}
\hline \multirow{4}{*}{$\begin{array}{l}\text { Biochemical Oxygen Demand } \\
\text { Total Organic Carbon } \\
\text { Sulfate }\end{array}$} & \multicolumn{2}{|c|}{$\begin{array}{l}\text { As Received } \\
\text { Result }\end{array}$} & \multirow{2}{*}{$\begin{array}{l}\text { Method } \\
\text { RL } \\
2\end{array}$} & \multirow{2}{*}{$\begin{array}{l}\begin{array}{l}\text { Method } \\
\text { Reference }\end{array} \\
\text { SM } 5210-B\end{array}$} & \multicolumn{4}{|c|}{$\begin{array}{l}\text { Date } \\
\text { Analyzed }\end{array}$} & \multirow{2}{*}{$\begin{array}{l}\text { Analyst } \\
\text { Shannon }\end{array}$} \\
\hline & 2.62 & $\mathrm{mg} / 1$ & & & 7 & Dec & 06 & $8: 45$ & \\
\hline & 8.4 & $\mathrm{mg} / \mathrm{I}$ & 0.5 & SMS310-C & 11 & Dec & 06 & $8: 30$ & Wayne \\
\hline & 2390 & $\mathrm{mg} / 1$ & 5.00 & EPA 375.4 & 12 & Dec & 06 & $15: 31$ & Morgan \\
\hline Nitrate-Nitrite as $\mathrm{N}$ & $<0.1$ & $\mathrm{mg} / 1$ & 0.10 & 353.2 & 13 & Dec & 06 & $9: 00$ & Morgan \\
\hline Ammonia-Nitrogen as $\mathrm{N}$ & 0.79 & $\mathrm{mg} / \mathrm{l}$ & 0.10 & EPA 350.1 & 15 & Dec & 06 & $10: 00$ & Morgan \\
\hline Phosphorus as p - Total & $<0.1$ & $\mathrm{mg} / \mathrm{I}$ & 0.10 & EPA 365.1 & 11 & Dec & 06 & $12: 00$ & Morgan \\
\hline Chemical Oxygen Demand & 13.3 & $\mathrm{mg} / \mathrm{I}$ & 1.0 & $\mathrm{HACH} 8000$ & 8 & Dec & 06 & $8: 30$ & wayne \\
\hline Iron - Tota 1 & 20.0 & $\mathrm{mg} / \mathrm{l}$ & 0.10 & 6010 & 21 & Dec & 06 & $9: 12$ & Stacy \\
\hline Manganese - Total & 0.71 & $\mathrm{mg} / 1$ & 0.05 & 6010 & 21 & Dec & 06 & $9: 12$ & stacy \\
\hline Iron - Dissolved & 0.80 & $\mathrm{mg} / 1$ & 0.10 & 6010 & 14 & Dec & 06 & $9: 41$ & stacy \\
\hline Manganese - Dissolved & 0.38 & $\mathrm{mg} / \mathrm{l}$ & 0.05 & 6010 & 14 & Dec & 06 & $9: 41$ & stacy \\
\hline
\end{tabular}

Approved by:

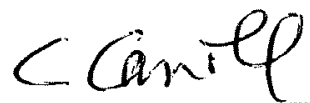

RI = Method Reporting linit

Elevated "Less Than Result" $(<): e$ = Due to sample matrix

$\#$ - Due to sample concentration

+ - Due to extract volume 
Jarda Solc

UND-Energy \& Environmental

15 N. $23 r d$ st.

Grand Forks ND 58201

Sample Description: MW- 6

Sample Site: Watford City
Page: 1 of 1

Report Date: 22 Dec 06

Lab Number: $06-$ W5632

Work order \#: 82-2656

Account \#: 007033

Date Sampled: 6 Dec $06 \quad 13: 55$

Date Received: 7 Dec 06 8:10

PO \#: CSI Watford

\begin{tabular}{|c|c|c|c|c|c|c|c|c|c|}
\hline \multirow[b]{2}{*}{ Biochemical oxygen Demand } & \multicolumn{2}{|c|}{$\begin{array}{l}\text { As Received } \\
\text { Result }\end{array}$} & \multirow{2}{*}{$\frac{\begin{array}{l}\text { Method } \\
\text { RL }\end{array}}{2}$} & \multirow{2}{*}{$\begin{array}{l}\begin{array}{l}\text { Method } \\
\text { Reference }\end{array} \\
\text { SM } 5210-B\end{array}$} & \multicolumn{4}{|c|}{$\begin{array}{l}\text { Date } \\
\text { Analyzed }\end{array}$} & \multirow{2}{*}{$\frac{\text { Analyst }}{\text { Shannon }}$} \\
\hline & 2.74 & $\mathrm{mg} / \mathrm{I}$ & & & 7 & Dec & 06 & $8: 45$ & \\
\hline Total Organic Carbon & 6.4 & $\mathrm{mg} / \mathrm{l}$ & 0.5 & $\operatorname{SMS} 310-\mathrm{C}$ & 11 & Dec & 06 & $8: 30$ & wayne \\
\hline Sulfate & 988 & $\mathrm{mg} / 1$ & 5.00 & EPA 375.4 & 12 & Dec & 06 & $15: 31$ & Morgan \\
\hline Nitrate-Nitrite as $N$ & 0.20 & $\mathrm{mg} / 1$ & 0.10 & 353.2 & 13 & Dec & 06 & $9: 00$ & Morgan \\
\hline Ammonia-Nitrogen as $\mathrm{N}$ & 0.89 & $\mathrm{mg} / \mathrm{I}$ & 0.10 & EPA 350.1 & 15 & Dec & 06 & $10: 00$ & Morgan \\
\hline Phosphorus as $\mathrm{P}$ - Total & $<0.1$ & $\mathrm{mg} / \mathrm{l}$ & 0.10 & EPA 365.1 & 11 & Dec & 06 & $12: 00$ & Morgan \\
\hline Chemical oxygen Demand & 42.5 & $\mathrm{mg} / 1$ & 1.0 & HACH 8000 & 8 & Dec & 06 & $8: 30$ & Wayne \\
\hline Iron - Tota1 & 28.1 & $\mathrm{mg} / 1$ & 0.10 & 6010 & 21 & Dec & 06 & $9: 12$ & stacy \\
\hline Manganese - Total. & 0.85 & $\mathrm{mg} / \mathrm{I}$ & 0.05 & 6010 & 21 & Dec & 06 & $9: 12$ & Stacy \\
\hline Iron - Dissolved & 6.48 & $\mathrm{mg} / \mathrm{I}$ & 0.10 & 6010 & 14 & Dec & 06 & $9: 41$ & stacy \\
\hline Manganese - Dissolved & 0.73 & $\mathrm{mg} / \mathrm{I}$ & 0.05 & 6010 & 14 & Dec & 06 & $9: 41$ & stacy \\
\hline
\end{tabular}

Approved by: 
Jarda Solc

UND-Energy \& Environmental

15 N. 23 rd st.

Grand Forks ND 58201

Sample Description: MW-8

Sample site: Watford City
Page: 1 of 1

Report Date: 22 Dec 06

Lab Number: 06-W5633

work Order \#: 82-2656

Account \#: 007033

Date Sampled: 6 Dec $0613: 30$

Date Received: 7 Dec 06 8:10

PO \#: CSI watford

\begin{tabular}{|c|c|c|c|c|c|c|c|c|c|}
\hline \multirow[b]{2}{*}{ Biochemical Oxygen Demand } & \multicolumn{2}{|c|}{$\begin{array}{l}\text { As Received } \\
\text { Result }\end{array}$} & \multirow{2}{*}{$\begin{array}{l}\begin{array}{l}\text { Method } \\
\text { RL. }\end{array} \\
2\end{array}$} & \multirow{2}{*}{$\begin{array}{l}\begin{array}{l}\text { Method } \\
\text { Reference }\end{array} \\
\text { SM } 5210-\mathrm{B}\end{array}$} & \multicolumn{4}{|c|}{$\begin{array}{l}\text { Date } \\
\text { Analyzed }\end{array}$} & \multirow{2}{*}{ Analyst } \\
\hline & $<2$ & $\mathrm{mg} / 1$ & & & 7 & Dec & 06 & $8: 45$ & \\
\hline Total organic Carbon & 27.0 & $\mathrm{mg} / 1$ & 0.5 & SM5310 $\sim \mathrm{C}$ & 11 & Dec & 06 & $8: 30$ & Wayne \\
\hline Sulfate & 13200 & $\mathrm{mg} / \mathrm{l}$ & 5.00 & EPA 375.4 & 12 & Dec & 06 & $15: 31$ & Morgan \\
\hline Nitrate-Nitrite as $\mathrm{N}$ & $<0.1$ & $\mathrm{mg} / 1$ & 0.10 & 353.2 & 13 & Dec & 06 & $9: 00$ & Morgan \\
\hline Ammonia-Nitrogen as $\mathrm{N}$ & 0.11 & $\mathrm{mg} / 1$ & 0.10 & EPA 350.1 & 15 & Dec & 06 & $10: 00$ & Morgan \\
\hline Phosphorus as p - Total & $<0.1$ & $\mathrm{mg} / \mathrm{l}$ & 0.10 & $\mathrm{EPA} 365.1$ & 11 & Dec & 06 & $12: 00$ & Morgan \\
\hline Chemical oxygen Demand & 63.6 & $\mathrm{mg} / 1$ & 1.0 & HACH 8000 & 8 & Dec & 06 & $8: 30$ & wayne \\
\hline Iron - Total & 24.2 & $\mathrm{mg} / 1$ & 0.10 & 6010 & 21 & Dec & 06 & $9: 12$ & stacy \\
\hline Manganese - Total & 2.29 & $\mathrm{mg} / 1$ & 0.05 & 6010 & 21 & Dec & 06 & $9: 12$ & Stacy \\
\hline Iron - Dissolved & $<0.1$ & $\mathrm{mg} / 1$ & 0.10 & 6010 & 14 & Dec & 06 & $9: 41$ & Stacy \\
\hline Manganese - Dissolved & 0.08 & $\mathrm{mg} / \mathrm{l}$ & 0.05 & 6010 & 14 & Dec & 06 & $9: 41$ & stacy \\
\hline
\end{tabular}

Approved by : 
1126 N. Front St. - New Ulm, MN 56073 - 800-782-3557 - Fax 507-359-2890

Jarda solc

UND-Energy \& Environmental

$15 \mathrm{~N} .23 \mathrm{rd}$ st.

Grand Forks ND 58201

Sample Description: MW- 9

Sample site: Watford City
Page: 1 of 1

Report Date: 22 Dec 06

Lab Number: $06-$ W5634

work Order \#: 82-2656

Account \#: 007033

Date Sampled: 6 Dec 06 14:30

Date Received: 7 Dec 06 8:10

PO \# : CSI Watford

\begin{tabular}{|c|c|c|c|c|c|c|c|c|c|}
\hline \multirow{4}{*}{$\begin{array}{l}\text { Biochemical Oxygen Demand } \\
\text { Total organic Carbon } \\
\text { Sulfate }\end{array}$} & \multicolumn{2}{|c|}{$\begin{array}{l}\text { As Received } \\
\text { Result }\end{array}$} & \multirow{2}{*}{$\begin{array}{l}\text { Method } \\
\text { RL } \\
2\end{array}$} & \multirow{2}{*}{$\begin{array}{l}\text { Method } \\
\text { Reference }\end{array}$} & \multicolumn{4}{|c|}{$\begin{array}{l}\text { Date } \\
\text { Analyzed }\end{array}$} & \multirow{2}{*}{$\begin{array}{l}\text { Analyst } \\
\text { Shannon }\end{array}$} \\
\hline & 3.32 & $\mathrm{mg} / 1$ & & & 7 & Dec & 06 & $8: 45$ & \\
\hline & 6.4 & $\mathrm{mg} / \mathrm{l}$ & 0.5 & SM5310-C & 11 & Dec & 06 & $8: 30$ & Wayne \\
\hline & 1490 & $\mathrm{mg} / \mathrm{l}$ & 5.00 & EPA 375.4 & 12 & Dec & 06 & $15: 31$ & Morgan \\
\hline Nitrate-Nitrite as $\mathrm{N}$ & $<0.1$ & $\mathrm{mg} / \mathrm{l}$ & 0.10 & 353.2 & 13 & Dec & 06 & $9: 00$ & Morgan \\
\hline Ammonia-Nitrogen as $\mathrm{N}$ & 1.64 & $\mathrm{mg} / \mathrm{I}$ & 0.10 & EPA 350.1 & 15 & Dec & 06 & $10: 00$ & Morgan \\
\hline Phosphorus as p - Total. & $<0.1$ & $\mathrm{mg} / \mathrm{l}$ & 0.10 & EPA 365.1 & 11 & Dec & 06 & $12: 00$ & Morgan \\
\hline Chemical oxygen Demand & 29.5 & $\mathrm{mg} / 1$ & 1.0 & $\mathrm{HACH} 8000$ & 8 & Dec & 06 & $8: 30$ & Wayne \\
\hline Iron - Total. & 26.9 & $m g / 1$ & 0.10 & 6010 & 21 & Dec & 06 & $9: 12$ & Stacy \\
\hline Manganese - Total & 1.03 & $\mathrm{mg} / \mathrm{l}$ & 0.05 & 6010 & 21 & Dec & 06 & $9: 12$ & stacy \\
\hline Iron - Dissolved & 8.42 & $\mathrm{mg} / 1$ & 0.10 & 6010 & 14 & Dec & 06 & $9: 41$ & stacy \\
\hline Manganese - Dissolved & 0.95 & $\mathrm{mg} / 1$ & 0.05 & 6010 & 14 & Dec & 06 & $9: 41$ & stacy \\
\hline
\end{tabular}

Approved by : 
1126 N. Front St. - New Ulm, MN 56073 - 800-782-3557 - Fax 507-359-2890

Jarda Solc

UND-Energy \& Environmental

1.5 N. 23 rd St.

Grand Forks ND 58201

Sample Description: Cherry Creek

Sample site: Watford city

As Received

Result

$\begin{array}{ll}7.27 & \mathrm{mg} / 1 \\ 22.0 & \mathrm{mg} / 1 \\ 1160 & \mathrm{mg} / 1 \\ <0.1 & \mathrm{mg} / 1 \\ <0.1 & \mathrm{mg} / 1 \\ 0.22 & \mathrm{mg} / 1 \\ 57.1 & \mathrm{mg} / 1 \\ 1.57 & \mathrm{mg} / 1 \\ 0.18 & \mathrm{mg} / 1 \\ <0.1 & \mathrm{mg} / 1 \\ <0.05 & \mathrm{mg} / 1\end{array}$

Biochemical Oxygen Demand

Total Organic Carbon

Sulfate

Nitrate-Nitrite as $\mathrm{N}$

Ammonia-Nitrogen as $\mathrm{N}$

Phosphorus as $p$ - Total

Chemical Oxygen Demand

Iron - rotal

Manganese - Tota 1

Iron - Dissolved

Manganese - Dissolved
Page: 1 of 1

Report Date: 22 Dec 06

Lab Number: 06-W5635

Work Order \#: 82-2656

Account \#: 007033

Date Sampled: 6 Dec 06 11:45

Date Received: 7 Dec 06 8:10

PO \#: CSI watford

\begin{tabular}{llllll}
$\begin{array}{l}\text { Method } \\
\text { RL }\end{array}$ & $\begin{array}{l}\text { Method } \\
\text { Reference }\end{array}$ & $\begin{array}{l}\text { Date } \\
\text { Analyzed }\end{array}$ & Analyst \\
\hline 2 & SM $5210-\mathrm{B}$ & 7 Dec 06 & $8: 45$ & Shannon \\
0.5 & SM5310-C & 11 DeC 06 & $8: 30$ & Wayne \\
5.00 & EPA 375.4 & 12 DeC 06 & $15: 31$ & Morgan \\
0.10 & 353.2 & 13 Dec 06 & $9: 00$ & Morgan \\
0.10 & EPA 350.1 & 15 DeC 06 & $10: 00$ & Morgan \\
0.10 & EPA 365.1 & 11 DeC 06 & $12: 00$ & Morgan \\
1.0 & HACH 8000 & 21 DeC 06 & $8: 30$ & Wayne \\
0.10 & 6010 & 21 DeC 06 & $9: 12$ & Stacy \\
0.05 & 6010 & 14 DeC 06 & $9: 41$ & Stacy \\
0.10 & 6010 & 14 DeC 06 & $9: 41$ & Stacy \\
0.05 & 6010 & & &
\end{tabular}

Approved by

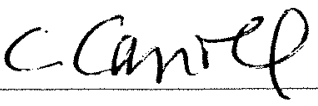

$\mathrm{RI}_{1}=$ Method Reporting Limit

Elevated "Less "Than Result" $|c|:$ : Due to sample matrix - Due to sample quantit 
1126 N. Front St. - New Ulm, MN 56073 - 80(1-782-3557 - Fax 507-359-2890

1411 S. 12th St. - Bismarck, ND 58502 - 800-279-6885 - Falx 701-258-9724

35 W. Lincoln Way - Nevada, IA 50201 - 800-362-(0855 - Fax 515-382-3885

Jarda Solc

UND-Energy \& Environmental

$15 \mathrm{~N} .23 \mathrm{rd}$ st.

Grand Forks ND 58201

Sample Description: Municipal Well

Sample site: Watford City

\section{Page: 1 of 1}

Report Date: 22 Dec 06

Lab Number: 06-W5636

work Order \#: $82-2656$

Account \#: 007033

Date Sampled: 6 Dec 06 15:35

Date Received: 7 Dec 06 8:10

PO \#: CSI watford

\begin{tabular}{|c|c|c|c|c|c|c|c|c|c|}
\hline \multirow{4}{*}{$\begin{array}{l}\text { Biochemical oxygen Demand } \\
\text { Total organic Carbon } \\
\text { Sulfate }\end{array}$} & \multicolumn{2}{|c|}{$\begin{array}{l}\text { As Received } \\
\text { Result }\end{array}$} & \multirow{2}{*}{$\begin{array}{l}\text { Method } \\
\text { RI. } \\
2\end{array}$} & \multirow{2}{*}{$\begin{array}{l}\begin{array}{l}\text { Method } \\
\text { Reference }\end{array} \\
\text { SM } 5210-B\end{array}$} & \multicolumn{4}{|c|}{$\begin{array}{l}\text { Date } \\
\text { Analyzed }\end{array}$} & \multirow{2}{*}{$\begin{array}{l}\text { Analyst } \\
\text { Matt }\end{array}$} \\
\hline & $<2$ & $\mathrm{mg} / 1$ & & & 7 & Dec & 06 & $13: 30$ & \\
\hline & 4.6 & $\mathrm{mg} / 1$ & 0.5 & SM5310-C & 11 & Dec & 06 & $8: 30$ & wayne \\
\hline & 517 & $m g / 1$ & 5.00 & EPA 375.4 & 12 & Dec & 06 & $15: 31$ & Morgan \\
\hline Nitrate-Nitrite as $\mathrm{N}$ & $<0.1$ & $\mathrm{mg} / 1$ & 0.10 & 353.2 & 13 & Dec & 06 & $9: 00$ & Morgan \\
\hline Ammonia-Nitrogen as $\mathrm{N}$ & 0.61 & $\mathrm{mg} / 1$ & 0.10 & EPA 350.1 & 15 & Dec & 06 & $10: 00$ & Morgan \\
\hline Phosphorus as P - Total & $<0.1$ & $\mathrm{mg} / \mathrm{I}$ & 0.10 & EPA 365.1 & 11 & Dec & 06 & $12: 00$ & Morgan \\
\hline Chemical Oxygen Demand & 14.9 & $\operatorname{mg} / 1$ & 1.0 & $\mathrm{HACH} 8000$ & 8 & Dec & 06 & $8: 30$ & wayne \\
\hline Iron - Total & 1.50 & $\mathrm{mg} / 1$ & 0.10 & 6010 & 21 & Dec & 06 & $9: 12$ & Stacy \\
\hline Manganese - Total & 0.10 & $\mathrm{mg} / 1$ & 0.05 & 6010 & 21 & Dec & 06 & $9: 12$ & stacy \\
\hline Iron - Dissolved & 1.38 & $\mathrm{mg} / \mathrm{l}$ & 0.10 & 6010 & 14 & Dec & 06 & $9: 41$ & Stacy \\
\hline Manganese - Dissolved & 0.10 & $\mathrm{mg} / 1$ & 0.05 & 6010 & 14 & Dec & 06 & $9: 41$ & stacy \\
\hline
\end{tabular}

Approved by :

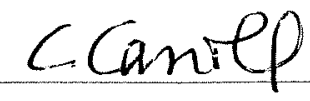

$\mathrm{RL}_{2}=$ Method Reporting timit

Elevated "tuess Than Result" (<): (1) : Due to sample matrix = Due to sarlple quantity 
Jarda Solc

UND-Energy \& Environmental

$15 \mathrm{~N} .23 \mathrm{rd}$ St.

Grand Forks ND 58201

Sample Description: MW-11

Sample site: Watford City
Page: 1 of 1

Report Date: 22 Dec 06

Lab Number: $06-$ W5637

work Order \#: $82-2656$

Account \#: 007033

Date Sampled: 6 Dec $06 \quad 10: 15$

Date Received: 7 Dec 06 8:10

PO \#: CSI Watford

\begin{tabular}{|c|c|c|c|c|c|c|c|c|c|}
\hline & \multicolumn{2}{|c|}{$\begin{array}{l}\text { As Received } \\
\text { Result }\end{array}$} & \multirow{2}{*}{$\begin{array}{l}\text { Method } \\
\text { RL } \\
2\end{array}$} & \multirow{2}{*}{$\begin{array}{l}\begin{array}{l}\text { Method } \\
\text { Reference }\end{array} \\
\text { SM } 5210-\mathrm{B}\end{array}$} & \multicolumn{4}{|c|}{$\begin{array}{l}\text { Date } \\
\text { Analyzed }\end{array}$} & \multirow{2}{*}{$\frac{\text { Analyst }}{\text { Matt }}$} \\
\hline Biochemical oxygen Demand & 60.0 & $\mathrm{mg} / 1$ & & & 7 & Dec & 06 & $13: 30$ & \\
\hline Total organic Carbon & 162 & $\mathrm{mg} / 1$ & 0.5 & SM5310-C & 11 & Dec & 06 & $8: 30$ & wayne \\
\hline sulfate & 5800 & $\mathrm{mg} / 1$ & 5.00 & EPA 375.4 & 12 & Dec & 06 & $15: 31$ & Morgan \\
\hline Nitrate-Nitrite as $N$ & 0.10 & $m g / 1$ & 0.10 & 353.2 & 13 & Dec & 06 & $9: 00$ & Morgan \\
\hline Ammonia-Nitrogen as $\mathrm{N}$ & 1.40 & $\mathrm{mg} / 1$ & 0.10 & EPA 350.1 & 15 & Dec & 06 & $10: 00$ & Morgan \\
\hline Phosphorus as p - Total & 0.53 & $\mathrm{mg} / \mathrm{l}$ & 0.10 & EPA 365.1 & 11 & Dec & 06 & $12: 00$ & Morgan \\
\hline Chemical oxygen Demand & 458 & $\mathrm{mg} / 1$ & 1.0 & HACH 8000 & 8 & Dec & 06 & $8: 30$ & wayne \\
\hline Iron - Total & 30.0 & $\mathrm{mg} / 1$ & 0.10 & 6010 & 21. & Dec & 06 & $9: 12$ & stacy \\
\hline Manganese - Total & 2.21 & $\mathrm{mg} / 1$ & 0.05 & 6010 & 21 & Dec & 06 & $9: 12$ & Stacy \\
\hline Iron - Dissolved & 2.60 & $\mathrm{mg} / \mathrm{I}$ & 0.10 & 6010 & 14 & Dec & 06 & $9: A 1$ & Stacy \\
\hline Manganese - Dissolved & 2.00 & $\mathrm{mg} / \mathrm{l}$ & 0.05 & 6010 & 14 & Dec & 06 & $9: 41$ & stacy \\
\hline
\end{tabular}

Approved by: 
Jarda Solc

UND-Energy \& Environmental

$15 \mathrm{~N} .23 \mathrm{rd} \mathrm{st}$.

Grand Forks ND 58201

Sample Description: MW-13

Sample Site: Watford City
Page: 1 of 1

Report Date: 22 Dec 06

Lab Number: 06-W5638

Work Order \#: 82-2656

Account \#: 007033

Date Sampled: 6 Dec 06 9:30

Date Received: 7 Dec 06 8:10

PO \#: CSI Watford

\begin{tabular}{|c|c|c|c|c|c|c|c|c|c|}
\hline \multirow[b]{2}{*}{ Biochemical oxygen Demand } & \multicolumn{2}{|c|}{$\begin{array}{l}\text { As Received } \\
\text { Result }\end{array}$} & \multirow{2}{*}{$\begin{array}{l}\text { Method } \\
\text { RI } \\
2\end{array}$} & \multirow{2}{*}{$\begin{array}{l}\text { Method } \\
\text { Reference }\end{array}$} & \multicolumn{4}{|c|}{$\begin{array}{l}\text { Date } \\
\text { Analyzed }\end{array}$} & \multirow{2}{*}{ Arralyst } \\
\hline & 120 & $\mathrm{mg} / \mathrm{I}$ & & & 7 & Dec & 06 & $13: 30$ & \\
\hline Total Organic Carbon & 55.0 & $\mathrm{mg} / 1$ & 0.5 & SM53 $10-C$ & 11 & Dec & 06 & $8: 30$ & wayne \\
\hline Sulfate & 124 & $\mathrm{mg} / \mathrm{I}$ & 5.00 & EPA 375.4 & 12 & Dec & 06 & $15: 31$ & Morgan \\
\hline Nitrate-Nitrite as $N$ & 0.11 & $\operatorname{mg} / 1$ & 0.10 & 353.2 & 13 & Dec & 06 & $9: 00$ & Morgan \\
\hline Ammonia-Nitrogen as $\mathrm{N}$ & 0.22 & $\mathrm{mg} / 1$ & 0.10 & EPA 350.1 & 15 & Dec & 06 & $10: 00$ & Morgan \\
\hline Phosphorus as p - Total & 0.59 & $\mathrm{mg} / \mathrm{I}$ & 0.10 & EPA 365.1 & 11 & Dec & 06 & $12: 00$ & Morgan \\
\hline Chemical Oxygen Demand & 270 & $\mathrm{mg} / 1$ & 1.0 & HACH 8000 & 8 & Dec & 06 & $8: 30$ & wayne \\
\hline Iron - Total & 13.0 & $\mathrm{mg} / \mathrm{I}$ & 0.10 & 6010 & 21 & Dec & 06 & $9: 12$ & stacy \\
\hline Manganese - Total & 0.76 & $\mathrm{mg} / \mathrm{I}$ & 0.05 & 6010 & 21 & Dec & 06 & $9: 12$ & Stacy \\
\hline Iron - Dissolved & $<1$ & $\mathrm{mg} / 1$ & 0.10 & 6010 & 14 & Dec & 06 & $9: 41$ & stacy \\
\hline Manganese - Dissolved & $<0.5$ & $\mathrm{mg} / 1$ & 0.05 & 6010 & 14 & Dec & 06 & $9: 41$ & Stacy \\
\hline
\end{tabular}

Approved by: 


\author{
Jarda Solc \\ UND-Energy \& Environmental \\ $15 \mathrm{~N} .23 \mathrm{rd} \mathrm{st}$. \\ Grand Forks ND 58201
}

Sample Description: Rec-1

Sample Site: Watford City
Page: $\quad 1$ of 1

Report Date: 22 Dec 06

Lab Number: 06-W5639

work order \#: $82-2656$

Account \#: 007033

Date Sampled: 6 Dec 06 12:00

Date Received: 7 Dec 06 8:10

PO \#: CSI watford

\begin{tabular}{|c|c|c|c|c|c|c|c|c|c|}
\hline \multirow{4}{*}{$\begin{array}{l}\text { Biochemical Oxygen Demand } \\
\text { Total Organic Carbon } \\
\text { Sulfate }\end{array}$} & \multicolumn{2}{|c|}{$\begin{array}{l}\text { As Received } \\
\text { Result }\end{array}$} & \multirow{2}{*}{$\begin{array}{l}\text { Method } \\
\text { RL } \\
2\end{array}$} & \multirow{2}{*}{$\begin{array}{l}\begin{array}{l}\text { Method } \\
\text { Reference }\end{array} \\
\text { SM } 5210-B\end{array}$} & \multicolumn{4}{|c|}{$\begin{array}{l}\text { Date } \\
\text { Analyzed }\end{array}$} & \multirow{2}{*}{ Analyst } \\
\hline & 45.7 & $\mathrm{mg} / \mathrm{l}$ & & & 7 & Dec & 06 & $13: 30$ & \\
\hline & 144 & $\mathrm{mg} / \mathrm{l}$ & 0.5 & SMS310-C & 11 & Dec & 06 & $8: 30$ & Wayne \\
\hline & 7100 & $\mathrm{mg} / 1$ & 5.00 & EPA 375.4 & 12 & Dec & 06 & $15: 31$ & Morgan \\
\hline Nitrate-Nitrite as $\mathrm{N}$ & $<0.1$ & $\mathrm{mg} / 1$ & 0.10 & 353.2 & 13 & Dec & 06 & $9: 00$ & Morgan \\
\hline Ammonia-Nitrogen as $\mathrm{N}$ & 1.06 & $\mathrm{mg} / \mathrm{l}$ & 0.10 & EPA 350.1 & 15 & Dec & 06 & $10: 00$ & Morgan \\
\hline Phosphorus as P - Total & 0.38 & $\mathrm{mg} / 1$ & 0.10 & EPA 365.1 & 11 & Dec & 06 & $12: 00$ & Morgan \\
\hline Chemical Oxygen Demand & 437 & $\mathrm{mg} / 1$ & 1.0 & $\mathrm{HACH} 8000$ & 8 & Dec & 06 & $8: 30$ & Wayne \\
\hline Iron - Total & 16.5 & $\mathrm{mg} / 1$ & 0.10 & 6010 & 21 & Dec & 06 & $9: 12$ & stacy \\
\hline Manganese - Total & 1.63 & $\mathrm{mg} / 1$ & 0.05 & 6010 & 21 & Dec & 06 & $9: 12$ & stacy \\
\hline Iron - Dissolved & 2.10 & $\mathrm{mg} / 1$ & 0.10 & 6010 & 14 & Dec & 06 & $9: 41$ & stacy \\
\hline Manganese - Dissolved & 1.80 & $\mathrm{mg} / 1$ & 0.05 & 6010 & 14 & Dec & 06 & $9: 41$ & stacy \\
\hline
\end{tabular}

CLproved by : Cante

Rt. = Method Reporting Limit

Elevated "Less "Than Result" (c) a d o Due to sample matrix - Due to sample quantity

\# - Due to sample concentration

" = Due to extract volune

ND \# ND-00016

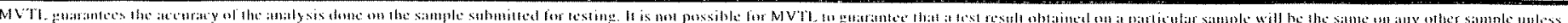

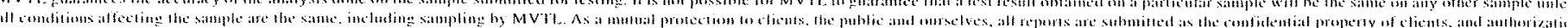

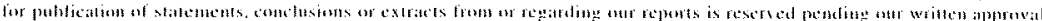

